\title{
Relaxation Dispersion NMR to reveal fast Dynamics in Brønsted Acid Catalysis: Influence of Sterics and H-bond strength on conformations and substrate hopping
}

N. Lokesh, Johnny Hioe, Johannes Gramüller and Ruth M. Gschwind*

Institute for organic chemistry,

University of Regensburg, D-93053 Regensburg, Germany

E-mail : ruth.gschwind@chemie.uni-regensburg.de

\section{Supplementary Material}

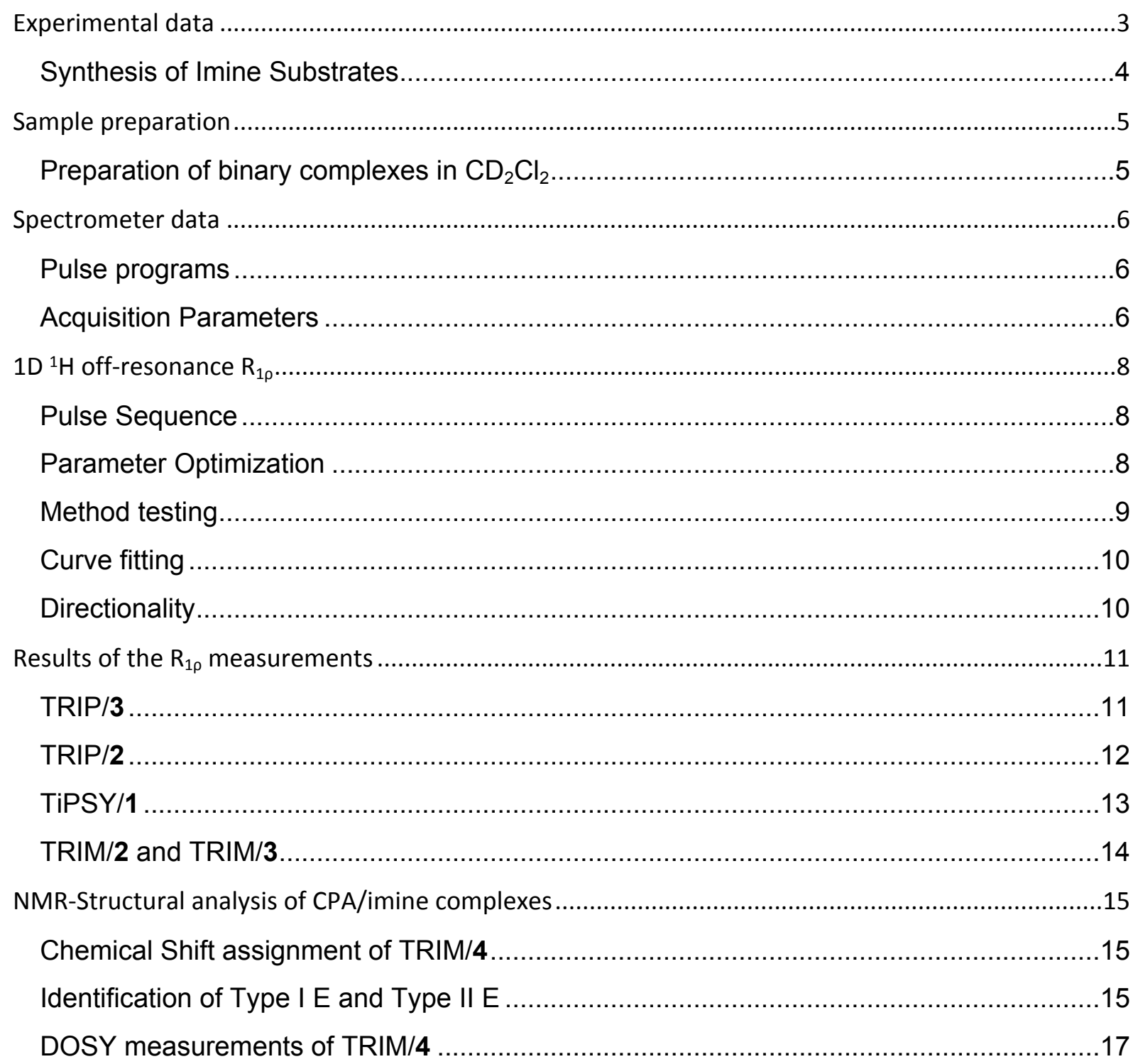


Estimation of the rotational correlation time of binary CPA/imine complexes .18

TiPSY/1 .19

Exchange with free imines .20

Computational Details 23

References. 54 


\section{Experimental data}

Deuterated solvents were purchased from Deutero or Sigma Aldrich. Where dry solvents were essential, $C_{2} \mathrm{Cl}_{2}$ was freshly distilled over $\mathrm{CaH}_{2}$ and Toluene was refluxed over $\mathrm{Na} /$ Benzophenone under argon atmosphere. The catalysts were purchased from Sigma Aldrich or STREM chemicals.

High-resolution mass spectra were measured by the central analytics division in the Institute of Organic Chemistry. Gas chromatography coupled with a mass selective detector was performed on an Agilent 6890N Network GC-System. 


\section{Synthesis of Imine Substrates}

The imines were prepared as described in the literature. ${ }^{1-4}$ The toluene was used either in p.A. quality or was dried by refluxing over sodium. The ${ }^{15} \mathrm{~N}$-enriched aniline (if used) was purchased from Euriso-top $\mathrm{GmbH}$ and Sigma Aldrich.

\section{(E)-N,1-bis(4-(tert-butyl)phenyl)ethan-1-imine [5]}

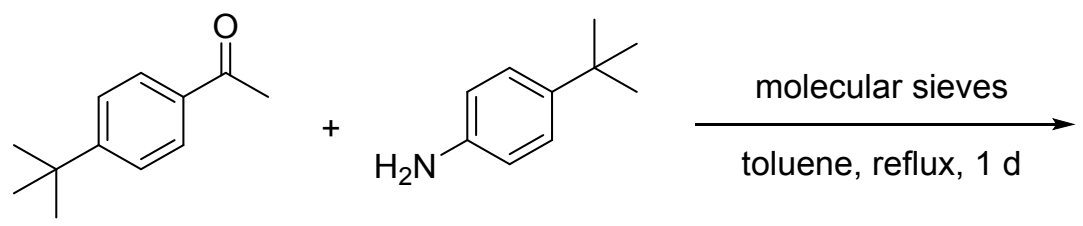<smiles>CC(=Nc1ccc(C(C)(C)C)cc1)c1ccc(C(C)(C)C)cc1</smiles>

Molecular sieves $4 \AA(4 \mathrm{~g})$ was weighed into a $50 \mathrm{~mL}$ Schlenk flask and dried with a heat gun at $350{ }^{\circ} \mathrm{C}$ for $30 \mathrm{~min}$ under reduced pressure. Under Argon flow, 4-(tert-butyl)aniline $(10.0 \mathrm{mmol}, 1.49 \mathrm{~g}, 1.60 \mathrm{~mL}, 1.0 \mathrm{eq})$ and 1-(4-(tert-butyl)phenyl)ethan-1-one $(10.0 \mathrm{mmol}$, $1.76 \mathrm{~g}, 1.83 \mathrm{~mL}, 1.0$ eq.) were added and dissolved in $20 \mathrm{~mL}$ anhydrous toluene. Under Argon flow, a reflux condenser was added to the setup and flushed with argon for 3 min. A drying tube filled with $\mathrm{CaCl}_{2}$ was added to the setup. The solution was refluxed for $24 \mathrm{~h}$. Afterward, the heating bath was removed, and the reaction mixture allowed to cool down. The orange mixture was filtrated, and the solvent was removed under reduced pressure to give an orangeyellow solid. The crude product was washed with methanol to give the product $(4.76 \mathrm{mmol}$, $1.46 \mathrm{~g}, 48 \%$ ) as yellow needles predominantly as its $E$-isomer (>99\% determined by ${ }^{1} \mathrm{H}$ NMR).

${ }^{1} \mathrm{H}-\mathrm{NMR}\left(400.1 \mathrm{MHz}, \mathrm{CD}_{2} \mathrm{Cl}_{2}\right) \delta_{\mathrm{H}}=7.91(\mathrm{~m}, 2 \mathrm{H}), 7.48(\mathrm{~m}, 2 \mathrm{H}), 7.37(\mathrm{~m}, 2 \mathrm{H}), 6.71(\mathrm{~m}, 2 \mathrm{H}), 2.21$ $(\mathrm{s}, 3 \mathrm{H}), 1.36(\mathrm{~s}, 9 \mathrm{H}), 1.34 \mathrm{ppm}(\mathrm{s}, 9 \mathrm{H})$.

${ }^{13}$ C-NMR $\left\{{ }^{1} \mathrm{H}\right\}(100.6 \mathrm{MHz}): \delta_{\mathrm{C}}=164.8,153.7,149.2,145.8,137.0,126.8,125.7,125.2,119.0$, $34.7,34.1,31.2,31.0,16.9 \mathrm{ppm}$.

HR-MS (ESI, $\mathrm{m} / \mathrm{z})$ : found $307.2289(\mathrm{M})+$ (calculated for 307.2300 for $\mathrm{C} 22 \mathrm{H} 29 \mathrm{~N})$; Diff(ppm) = $-1.92 \mathrm{ppm}$. 
Sample preparation

Preparation of binary complexes in $\mathrm{CD}_{2} \mathrm{Cl}_{2}$

The catalyst was dried for $30 \mathrm{~min}$ at $150^{\circ} \mathrm{C}$ under reduced pressure. Ketimine and catalyst were directly weighed into a $5 \mathrm{~mm}$ NMR tube under an inert argon atmosphere. $\mathrm{CD}_{2} \mathrm{Cl}_{2}(0.6 \mathrm{ml}$; freshly destilled over $\mathrm{CaH}_{2}$ ) and $1.0 \mathrm{ml}$ of tetramethylsilane atmosphere were added to the tube. The sample was stored in an $-80^{\circ} \mathrm{C}$ freezer. A $1: 1$ ratio of catalyst/ketimine was used for all samples unlike stated otherwise. A concentration of $0.25-100 \mathrm{mM}$ was used, as indicated specifically. 


\section{Spectrometer data}

NMR experiments were performed on Bruker Avance III HD $400 \mathrm{MHz}$ spectrometer, equipped with $5 \mathrm{~mm}$ BBO BB-1H/D probe head with Z-Gradients and a Bruker Avance III HD $600 \mathrm{MHz}$ spectrometer, equipped with a $5 \mathrm{~mm}$ CPPBBO BB-1H/19F. The temperature was controlled in the VT-experiments by BVT 3000 and BVTE 3900. For NMR measurements employing standard NMR solvents $5 \mathrm{~mm}$ NMR tubes were used, if not otherwise noted. NMR Data were processed, evaluated and plotted with TopSpin 3.2 software. Further plotting of the spectra was performed with Corel Draw X14 - X17 software. ${ }^{1} \mathrm{H},{ }^{13} \mathrm{C}$ chemical shifts were referenced to TMS or the respective solvent signals. The heteronuclei ${ }^{19} \mathrm{~F}$ and ${ }^{31} \mathrm{P}$ were referenced, employing $v(\mathrm{X})=v(\mathrm{TMS}) \times \Xi_{\text {reference }} / 100 \%$ according to Harris et al. ${ }^{5}$ The following frequency ratios and reference compounds were used: $\Xi\left({ }^{19} \mathrm{~F}\right)=94.094011\left(\mathrm{CCl}_{3} \mathrm{~F}\right), \equiv\left({ }^{31} \mathrm{P}\right)=40.480742$ $\left(\mathrm{H}_{3} \mathrm{PO}_{4}\right)$.

\section{Pulse programs}

All pulse programs used are standard Bruker NMR pulseprograms except for the $R_{1}$ measurements.

\section{Acquisition Parameters}

1H NMR: Pulse program: zg; Relaxation delay $=2-3 \mathrm{~s}$, Acquisition time $=2.48 \mathrm{~s}, \mathrm{SW}=$ $22.0 \mathrm{ppm}, \mathrm{TD}=64 \mathrm{k}, \mathrm{NS}=8-64 ; \mathrm{zg} 30$; Relaxation delay $=2 \mathrm{~s}$, Acquisition time $=2.48 \mathrm{~s}$, $\mathrm{SW}=22.0 \mathrm{ppm}, \mathrm{TD}=64 \mathrm{k}, \mathrm{NS}=8-64$;

2D- ${ }^{1} \mathrm{H},{ }^{1} \mathrm{H}$ NOESY: Pulse program: noesygpph/noesygpphpp; Relaxation delay $=5-8 \mathrm{~s}, \mathrm{NS}$ $=8-32$, mixing time $(D 8)=300.00 \mathrm{~ms} ; \mathrm{TD}=4096$; increments $=512-1 \mathrm{k}$;

2D-1 ${ }^{1},{ }^{1} \mathrm{H}$ COSY: Pulse program: cosygpqf; Relaxation delay $=5-8 \mathrm{~s}, \mathrm{NS}=8-32, \mathrm{TD}=$ 4096; increments $=512-1 \mathrm{k}$;

${ }^{13} \mathrm{C}$ NMR: Pulse program: zgpg30; Relaxation delay $=2.00 \mathrm{~s}$, Acquisition time $=0.80 \mathrm{~s}, \mathrm{SW}=$ $270.0 \mathrm{ppm}, \mathrm{TD}=64 \mathrm{k}, \mathrm{NS}=1 \mathrm{k}-2 \mathrm{k}$;

2D-1 ${ }^{1},{ }^{13} \mathrm{C}$ HSQC: Pulse program: hsqcedetgpsisp2.3; Relaxation delay = $4-8 \mathrm{~s}, \mathrm{NS}=8-32$, ${ }^{1} \mathrm{~J}_{\mathrm{XH}}=145 \mathrm{~Hz} ; \mathrm{TD}=4096$; increments = $512-1 \mathrm{k}$;

2D-1 $\mathrm{H},{ }^{13} \mathrm{C}$ HMBC: Pulse program: hmbcgplpndqf; Relaxation delay $=4-8 \mathrm{~s}, \mathrm{NS}=8-32,{ }^{1} \mathrm{~J}_{\mathrm{XH}}$ $=145 \mathrm{~Hz}, J_{X H}($ long range $)=10 \mathrm{~Hz} ; \mathrm{TD}=4096$; increments = $512-1 \mathrm{k}$;

2D-1 $\mathrm{H}^{31} \mathrm{P}$ HMBC: Pulse program: inv4gplrndqf; Relaxation delay $=4-8 \mathrm{~s}, \mathrm{NS}=8-32, \mathrm{TD}=$ 4096; increments $=256-1 \mathrm{k}$;

15N NMR: Pulse program: zg; Relaxation delay $=10.00 \mathrm{~s}$, Acquisition time $=0.54 \mathrm{~s}, \mathrm{SW}=$ $502.8 \mathrm{ppm}, \mathrm{TD}=32 \mathrm{k}, \mathrm{NS}=256-2048$;

2D- ${ }^{1} \mathrm{H},{ }^{15} \mathrm{~N}$ HMBC: Pulse program: inv4gplrndqf; Relaxation delay $=5-8 \mathrm{~s}, \mathrm{NS}=16-32$, delay for evolution of long range couplings $(D 6)=20.00 \mathrm{~ms}$; $T D=4096$; increments $=128-512$;

19F-NMR: Pulse program: zg30; Relaxation delay $=2-3 \mathrm{~s}$, Acquisition time $=11.60 \mathrm{~s}, \mathrm{SW}=$ $10.0 \mathrm{ppm}, \mathrm{TD}=128 \mathrm{k}, \mathrm{NS}=8-64$; 


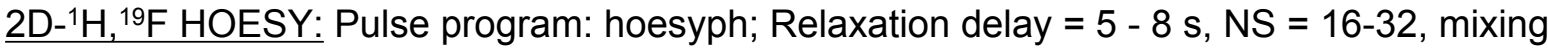
time $(\mathrm{D} 8)=500.00 \mathrm{~ms} ; \mathrm{TD}=4096$; increments $=1 \mathrm{k}$; 


\section{D ${ }^{1} \mathrm{H}$ off-resonance $\mathrm{R}_{1 \rho}$}

\section{Pulse Sequence}

The recent reported $1 \mathrm{D}{ }^{1} \mathrm{H}$ off-resonance $R_{1 \rho}$ pulse sequence is adopted with slight modification for our measurement. 6,7

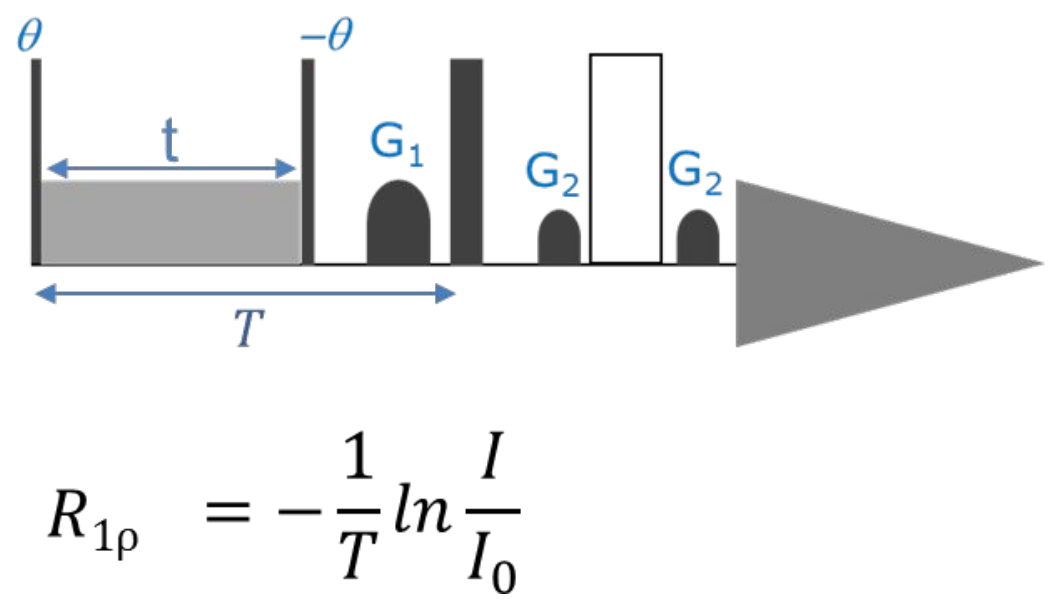

Figure 1: Applied pulse sequence and mathematical relation to measure $R_{1_{p}}$ experimenally. The applied angle $(\theta)$ is $35^{\circ}$. A spinlock period $(\mathrm{t})$ of $100 \mathrm{~ms}$ is applied. The filled (black) and unfilled rectanglur pulses respectively indicate $90^{\circ}$ and $180^{\circ}$ hard pulses. The gradient pulses of $G_{1}=70 \%$ and $G_{2}=40 \%$ of the maximum were applied. $I_{0}$ is the intensity without any spinlock period and I is the intensity with spinlock period measured over a multiple effective magnetic filed.

\section{Parameter Optimization}

The spinlock period $(\mathrm{t})$ was incremented in $10 \mathrm{~ms}$ steps with maximum spinlock power $(\approx 20$ $\mathrm{kHz}$ ). At each step, the temperature was monitored (sample heating can occur due to application of high intensity spinlock field over an extended time). Around $120 \mathrm{~ms}$, temperature oscillation was observed. To avoid any influence of temperature changes on the measurement, the spinlock period of $100 \mathrm{~ms}$ was applied in all our measurements.

The normal relaxation period (T-t) in the absene of the spinlock is tested for 3 delays (50 ms, $100 \mathrm{~ms}$ and $150 \mathrm{~ms}$ ). For all three different relaxation periods (T-t), we found similar exchange rates within the experimantal errors. To minimise signal loss due to relaxation, (T-t) of $50 \mathrm{~ms}$ was used in all our measurements. 


\section{Method testing}

To test the applicability of the $R_{1 \rho}$ method, we applied the above described pulse sequence with optimized parameters on the known peak in the spectrum (para-methyl protons of the hydrolyzed imine ketone part), which does not experience any chemical exchange process (on a ms- $\mu$ s timescale) and on the same proton for the CPA/imine complex which experiences chemical exchange. The measured rate for the hydrozyled ketone showed a nearly straight line (no offset-Lorentzian decay with increasing effective field $\omega_{\text {eff }}$ ), which was expected for non exchanging protons. For the same proton in the binary CPA/imine complex, an offsetLorentzian decay was observed, which demonstrates the presenence of chemical exchange on a ms- $\mu$ s timescale.
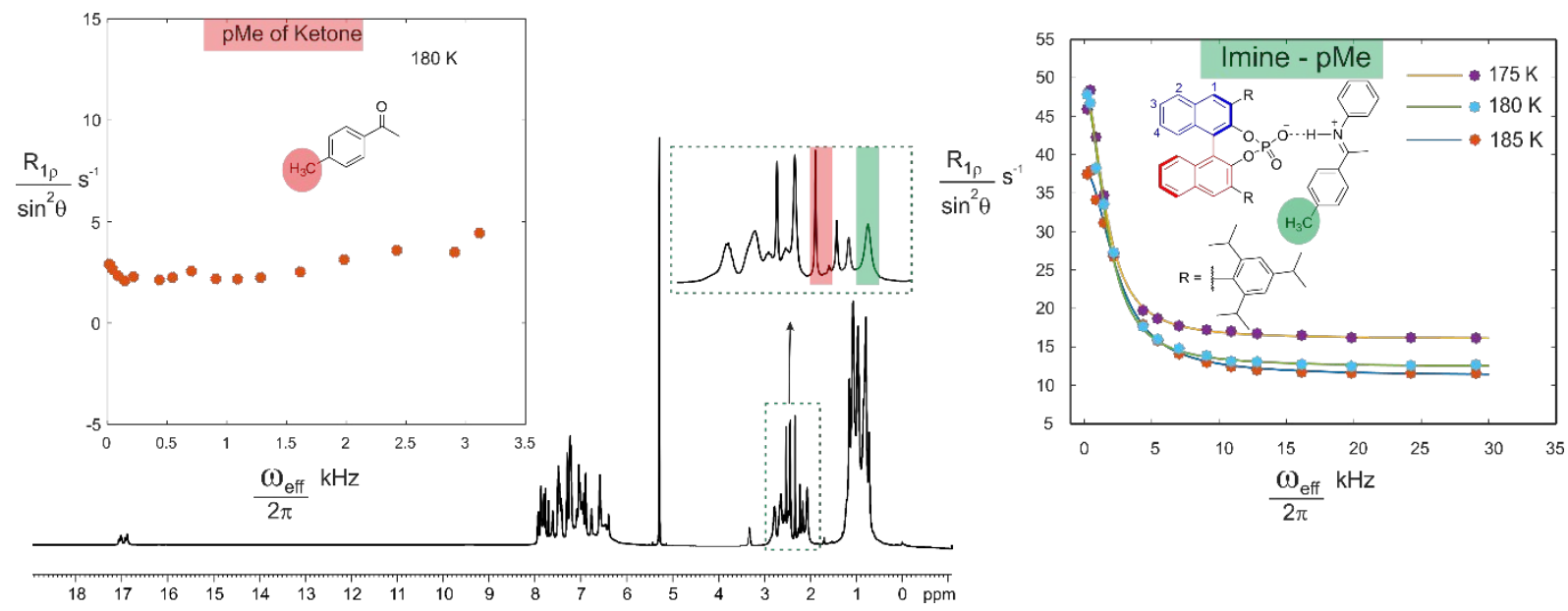

Figure 2: To test the method, we selected the proton peak of the $p$-methyl group in the hydrolyzed ketone moiety (red shaded) and the proton peak of the $p$-methyl imine in the TRIP/imine binary complex (green shaded) within the same sample (to maintain identical conditions). The hydrolyzed ketone does not undergo any chemical exchange on a ms- $\mu$ s time scale. The corresponding measured $\mathrm{R}_{1 \rho}$ data at $180 \mathrm{~K}$ with incremental effective field showed a near horizontol line, proving the absence of chemical exchange (left side). On the other hand, the imine inside the catalyst undergoes $E_{l} \rightleftharpoons E_{\| \mid}$exchange and thus the corresponding $R_{1 \rho}$ data at 185,180 and $175 \mathrm{~K}$ with incremental effective field showed offset-Lorentzian decays for the p-methyl protons in the TRIP/imine complex. This confirms the presence of chemical exchange process and hence validates our method for chemical exchange detection in ms- $\mu$ s range. 


\section{Curve fitting}

In order to analyze the $\mathrm{R}_{1 \rho}$ measurements, the signal intensities (integrals) are determined for every effective field strength $\omega_{\text {eff }}$ (i.e. every different spinlock power) and normalized to the reference intregral (spinlock power $=0 \mathrm{~W}$ ). Initially, to extract $k_{\mathrm{ex}}$, the measured experimental points are curve fitted to the following theoretically described equation by using MATLAB (see literature for further details ${ }^{8}$ ).

$$
\frac{R_{e f f}}{\sin ^{2} \theta}=R_{2}-R_{1}+\frac{(\Delta \omega)^{2} P_{A} P_{B} \tau e x}{(1+\underset{\tau e x \omega e f f}{2})}
$$

Here $\quad R_{2}$ is the tranverse relaxation rate constant

$R_{1}$ is the longitudinal relaxation rate constant

$\Phi=(\Delta \omega)^{2} P_{A} P_{B}$ tex encodes both chemical shift differnce and population of the minor $\left(P_{B}\right)$ and major $\left(P_{A}\right)$ exchange species

$\Phi$ and the average isotropic chemical shift $\Omega$ (in ppm) are related by

$$
\Phi=-\Omega^{2}+a \Omega+b
$$

Here,

$a=2 \Omega_{A}-\Delta \omega$

$b=\Omega_{A}\left(\Delta \omega-\Omega_{A}\right)$

These can be rearranged as

$$
\Delta \omega=\left(a^{2}+4 b\right)^{1 / 2} \text {, and } \Omega_{A, B}=\left[a \pm ;\left(a^{2}+4 b\right)^{1 / 2}\right] / 2
$$

$\Phi$ and $\Omega$ can be obtained experimentally at different temperatures. Assuming the chemicalshift difference $\Delta \omega$ between major (A) and minor (B) species is constant, one can calculate $\Delta \omega$. From $\Delta \omega$ value and $\Phi$ values, populations can be extractable.

\section{Directionality}

For the chemical exchange between $A$ and $B$, the exchange rate constant $k$ is: $k_{e x}=k_{A}+k_{B}$. By using populations and $\mathrm{k}_{\mathrm{ex}}$ one can extract rate constants $\mathrm{k}_{\mathrm{A}}$ and $\mathrm{k}_{\mathrm{B}}$ on both sides.

$$
\mathrm{A} \underset{k_{B}}{\stackrel{k_{A}}{\rightleftarrows}} \mathrm{B} \quad P_{A}=\frac{k_{B}}{k_{A}+k_{B}} \quad P_{B}=\frac{k_{A}}{k_{A}+k_{B}}
$$


If a temperature independence within the varied range of $10 \mathrm{~K}$ is assumed, then the coefficients $a$ and $b$ can be determined by least squares optimization. Subsequently, $\Delta \omega$ is calculated by $\Delta \omega=\left(a^{2}+4 b\right)^{1 / 2}$, and $\Omega_{\mathrm{A}, \mathrm{B}}=\left[a \pm ;\left(a^{2}+4 b\right)^{1 / 2}\right] / 2$. Once $\Delta \omega$ is known, $P_{\mathrm{A}}, P_{\mathrm{B}}, k_{\mathrm{A}}$ and $k_{\mathrm{B}}$ are obtained from $\Phi_{\mathrm{ex}}$ and $k_{\mathrm{ex}}$ at each temperature.

Hence, in order to seperate the populations and chemical shift difference from the term $\phi$, it requires additional experimental $R_{1 \rho}$ measurements at more than one temperature. Therefore we measured $R_{1 \rho}$ experiments for each sample at two or three temperatures $(185 \mathrm{~K}, 180 \mathrm{~K}$ and $175 \mathrm{~K}$ ).

Results of the $R_{1 \rho}$ measurements

$T R I P / 3$

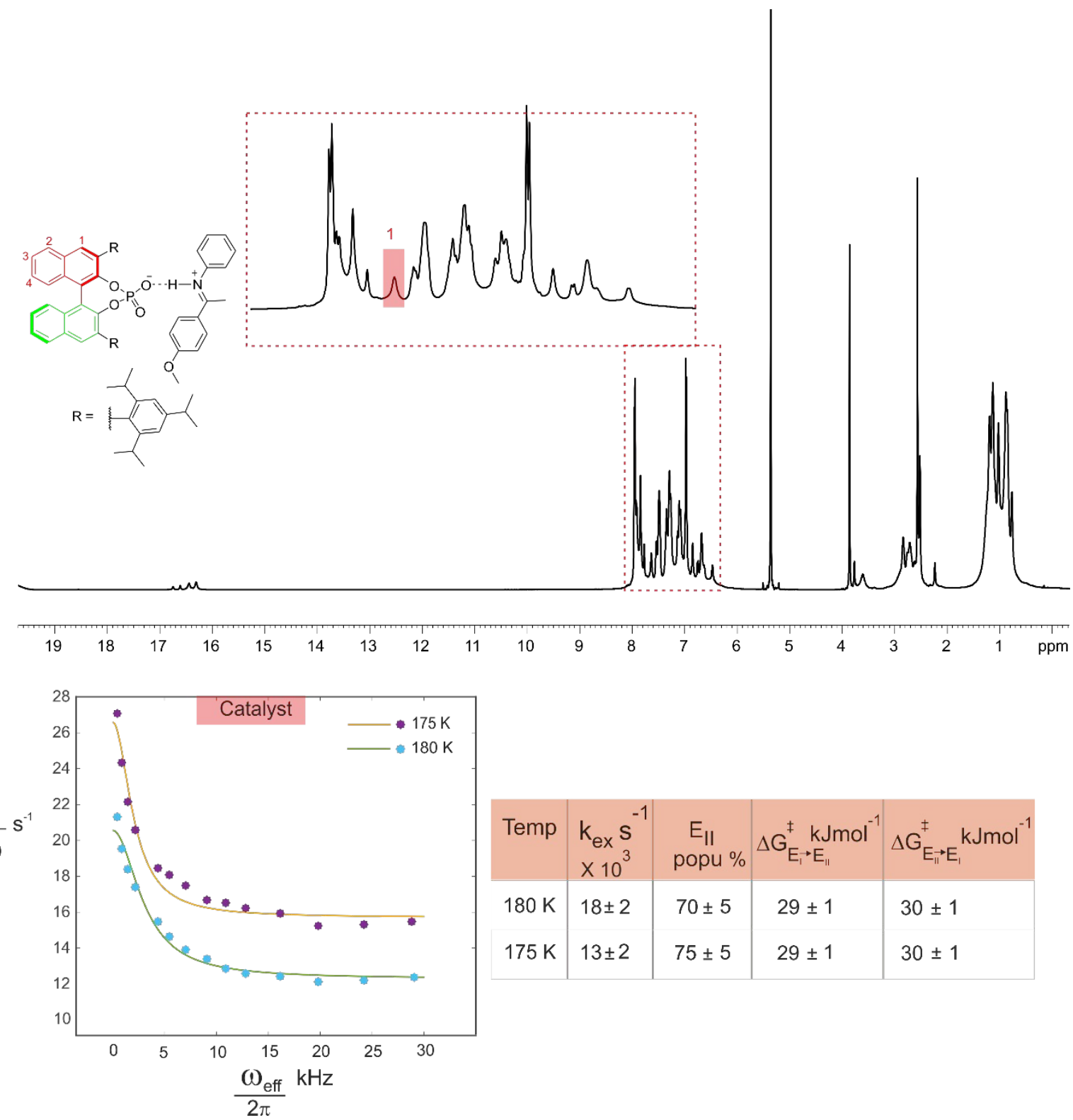

Figure 3: $R_{1 \rho}$ measurement for the TRIP/3 complex. The proton $\mathrm{H}_{1}$ of the catalyst (marked in the spectrum, red shaded) was selected as probe. The offset-Lorentzian decay indicates the 
pesence of chemical exchange $\left(k_{\mathrm{ex}} \approx 18000 \mathrm{~s}^{-1}\right.$ at $\left.180 \mathrm{~K}\right)$. With experimental data obtained at two different temperatures, the populations and directionality were extracted.

\section{$T R I P / 2$}

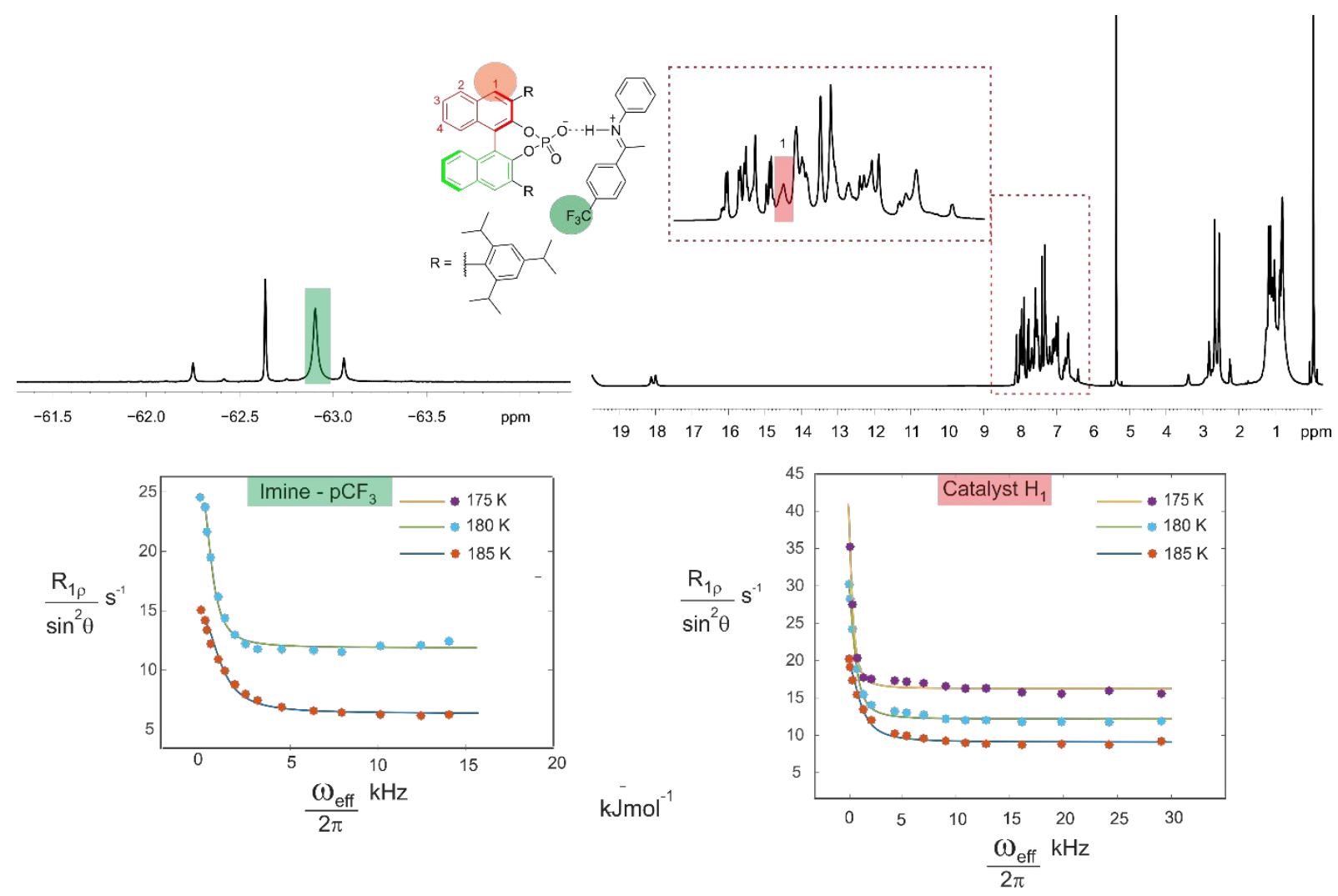

\begin{tabular}{|c|c|c|c|c|}
\hline Temp & $\begin{array}{l}\mathrm{k}_{\mathrm{ex}} \mathrm{s}^{-1} \\
\times 10^{3}\end{array}$ & $\begin{array}{c}\mathrm{E}_{\|} \\
\text {popu \% }\end{array}$ & $\Delta \mathrm{G}_{\mathrm{E}_{1} \cdot \mathrm{E}_{\|}}^{\ddagger} \mathrm{kJmol}^{-1}$ & $\Delta \mathrm{G}_{\mathrm{E}_{\|} \mathrm{E}_{1}}^{\ddagger} \mathrm{kJmol}^{-1}$ \\
\hline $185 \mathrm{~K}$ & $6 \pm 1$ & $76 \pm 5$ & $30 \pm 1$ & $32 \pm 1$ \\
\hline $180 \mathrm{~K}$ & $3.5 \pm 1$ & $88 \pm 5$ & $30 \pm 1$ & $32 \pm 1$ \\
\hline
\end{tabular}

\begin{tabular}{|c|c|c|c|c|}
\hline Temp & $\begin{array}{l}k_{e x} s^{-1} \\
\times 10^{3}\end{array}$ & $\begin{array}{l}\mathrm{E}_{\|} \\
\text {popu \% }\end{array}$ & $\Delta \mathrm{G}_{\mathrm{E}_{1} \rightarrow \mathrm{E}_{\|}}^{\ddagger} \mathrm{kJ}$ & $\Delta \mathrm{G}_{\mathrm{E}_{\|} \cdot \mathrm{E}_{1}}^{\ddagger} \mathrm{kJ} \mathrm{mol}^{-1}$ \\
\hline $185 \mathrm{~K}$ & $9 \pm 1$ & $74 \pm 5$ & $31 \pm 1$ & $33 \pm 1$ \\
\hline $180 \mathrm{~K}$ & $5 \pm 1$ & $78 \pm 5$ & $31 \pm 1$ & $33 \pm 1$ \\
\hline $175 \mathrm{~K}$ & $2.5 \pm 1$ & $83 \pm 5$ & $31 \pm 1$ & $33 \pm 1$ \\
\hline
\end{tabular}

Figure 4: $\mathrm{R}_{1 \rho}$ measurement for TRIP/2 complex. The proton $\mathrm{H}_{1}$ of the catalyst (shaded in red) and the fluorine signals of the imine- $\mathrm{CF}_{3}$ group (shaded in green) were selected as probes. The offset-Lorentzian decay indicates pesence of chemical exchange $\left(k_{\mathrm{ex}} \approx 3-5 \cdot 10^{3} \mathrm{~s}^{-1}\right.$ at 180 $\mathrm{K})$. With experimental data obtained at three different temperatures, the populations and directionality were extracted. 


\begin{tabular}{|c|c|c|c|c|}
\hline Temp K & $\begin{array}{l}k_{\mathrm{ex}} \mathrm{s}^{-1} \\
\times 10^{3}\end{array}$ & $E_{\| 1} \%$ & $\Delta \mathrm{G}_{E_{1} \rightarrow E_{\|}^{\ddagger}}^{\mathrm{KJ}} \mathrm{mol}^{\prime \prime}$ & $\Delta G_{E_{i \|} \rightarrow E_{1}}^{\ddagger} \mathrm{KJ}_{\mathrm{J}} \mathrm{mol}$ \\
\hline 185 & $3.0 \pm 1$ & $95 \pm 5$ & $32 \pm 1$ & $36 \pm 1$ \\
\hline 180 & $2.5 \pm 1$ & $96 \pm 4$ & $32 \pm 1$ & $36 \pm 1$ \\
\hline 175 & $2.5 \pm 2$ & $95 \pm 5$ & $31 \pm 1$ & $35 \pm 1$ \\
\hline
\end{tabular}
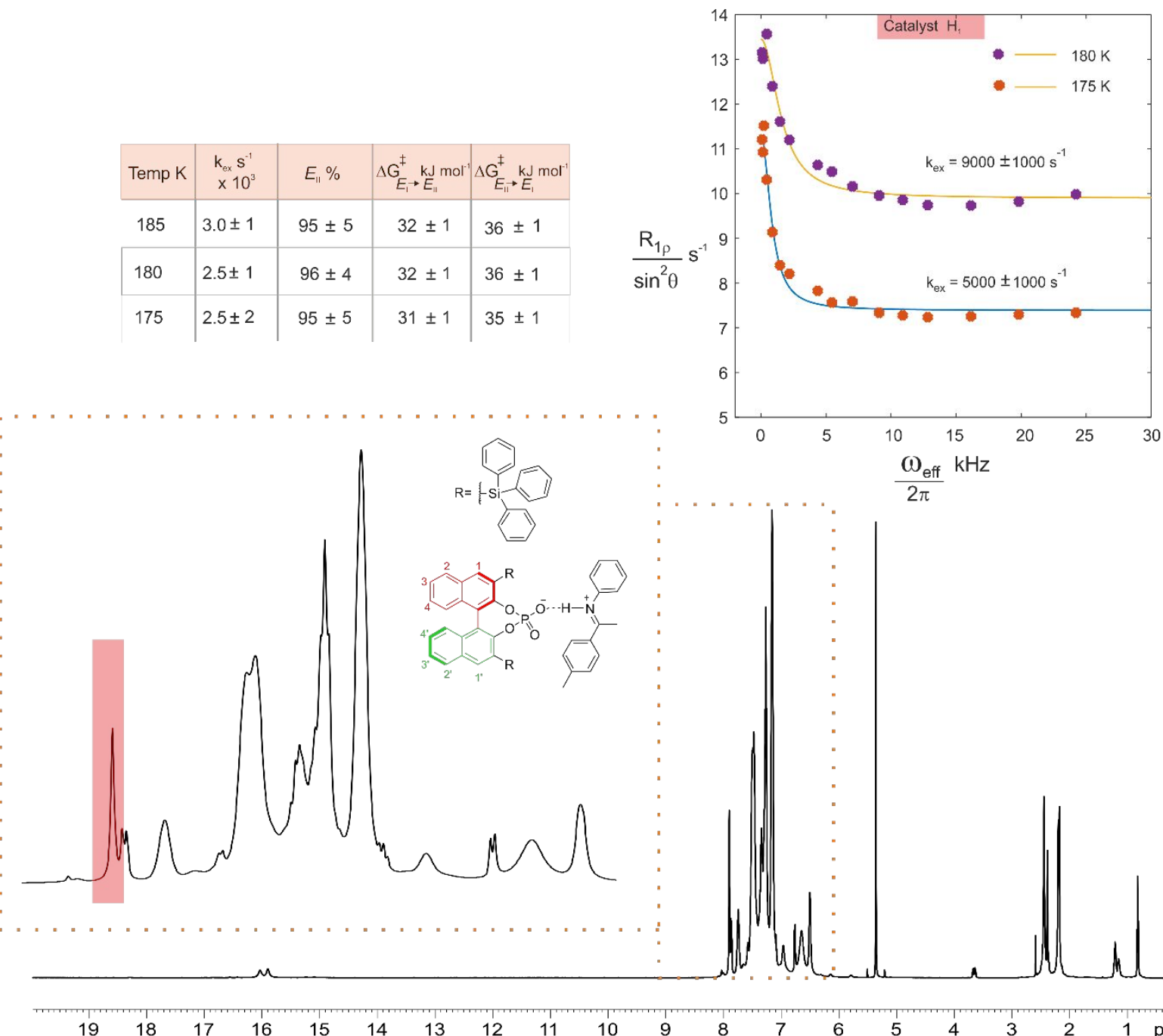

Figure 5: $R_{1 \rho}$ measurement for TiPSY/1 complex, (to avoid signal overlap, the sample was prepared with nearly exclusive population of the E-complex; see above for sample preparation). The proton $\mathrm{H}_{1}$ of the catalyst (shaded in red) was selected as probe. The offsetLorentzian decay indicates pesence of chemical exchange $\left(k_{\mathrm{ex}} \approx 9 \mathrm{ks}^{-1}\right.$ at $\left.180 \mathrm{~K}\right)$. 

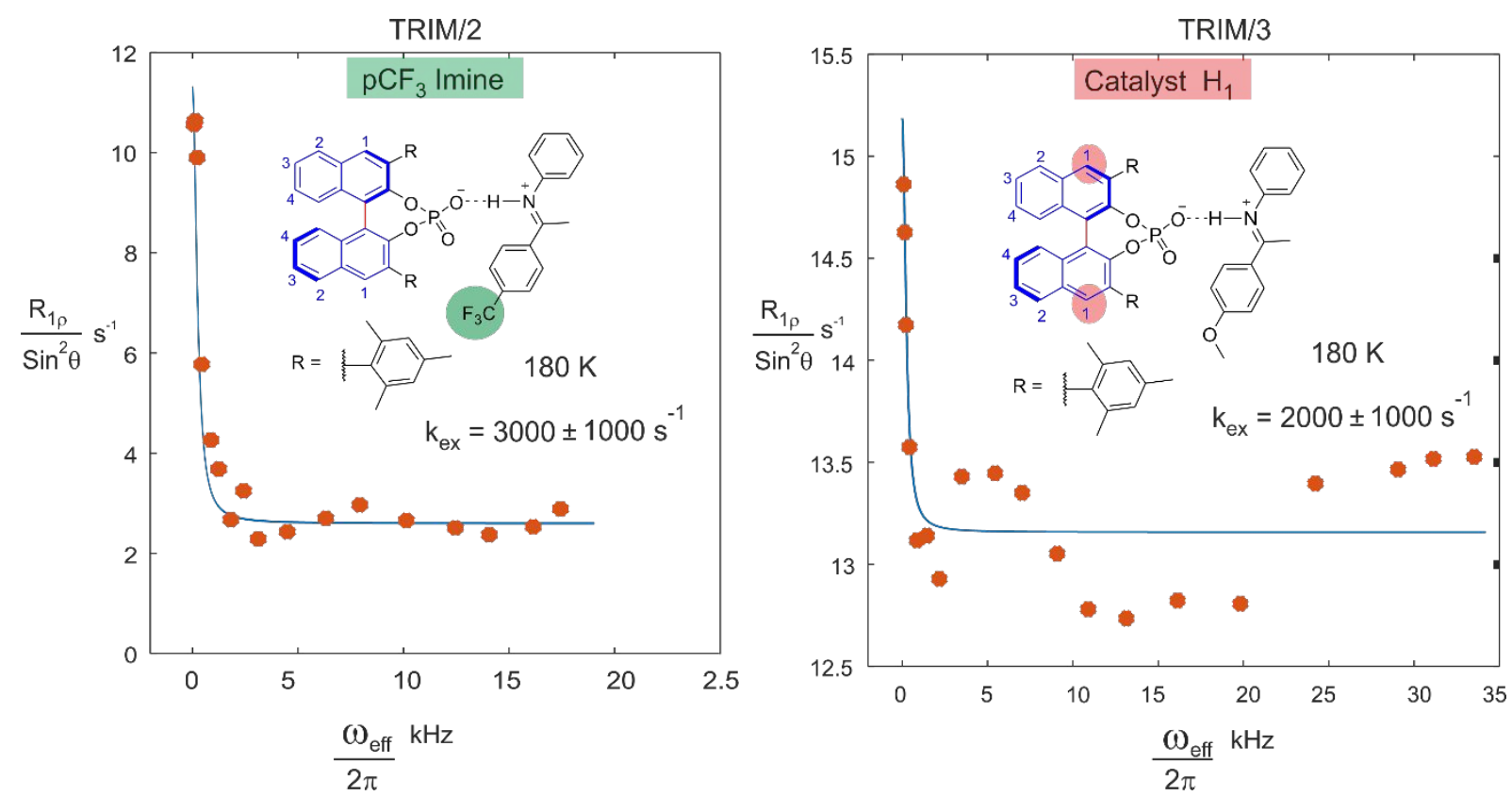

Figure 6: $R_{1 p}$ measurements for TRIM/2 and TRIM/3 complexes. For TRIM/2, the $\mathrm{CF}_{3}$ group of the imine was used as a probe (shaded in green) and for TRIM/3 the proton $\mathrm{H}_{1}$ of the catalyst (shaded in red) was selected as probe. The offset-Lorentzian decay in both system indicates the pesence of chemical exchange $\left(k_{\mathrm{ex}} \approx 2000-3000 \mathrm{~s}^{-1}\right.$ at $\left.180 \mathrm{~K}\right)$.

For TRIM/2 and TRIM/3, the offset-Lorentzian decay fit does not match the experimental decay as precisely as for the other systems. Hence, a larger experimental error is expected. However, the $R_{1 \rho}$ measurements for TRIM/2 and TRIM/3 complexes indicate, that similar exchange rates $\left(k_{\mathrm{ex}} \approx 2000-3000 \mathrm{~s}^{-1}\right.$ at $\left.180 \mathrm{~K}\right)$ are obtained for TRIM/1-3 regardless of the hydrogen bond strength. 
NMR-Structural analysis of CPA/imine complexes

The investigated complexes TRIP/1-3, TiPSY/1 and TRIM/1-3 were already described in the previous work. 2,9 The NMR-structural analysis of TRIM/4 is described below. In addition, the backbone splitting of TiPSY/1 is discussed in detail below.

\section{Chemical Shift assignment of TRIM/4}

The ${ }^{1} \mathrm{H}$ (black) and ${ }^{13} \mathrm{C}$ (green) chemical shifts (in ppm) of all investigated complexes were assigned with standard 2D NMR experiments $\left({ }^{1} \mathrm{H},{ }^{1} \mathrm{H}\right.$ COSY, ${ }^{1} \mathrm{H},{ }^{1} \mathrm{H}$ TOCSY, ${ }^{1} \mathrm{H},{ }^{1} \mathrm{H}$ NOESY, ${ }^{1} \mathrm{H},{ }^{13} \mathrm{C} \mathrm{HSQC},{ }^{1} \mathrm{H},{ }^{13} \mathrm{C} \mathrm{HMBC}$ ) at $180 \mathrm{~K}$. The ${ }^{31} \mathrm{P}$ (orange) chemical shift (in ppm) was assigned by ${ }^{1} \mathrm{H},{ }^{31} \mathrm{P} H M B C$. Due to signal overlap and lacking resolution and intensity of $2 \mathrm{D}$ correlations, not all signals could be assigned.
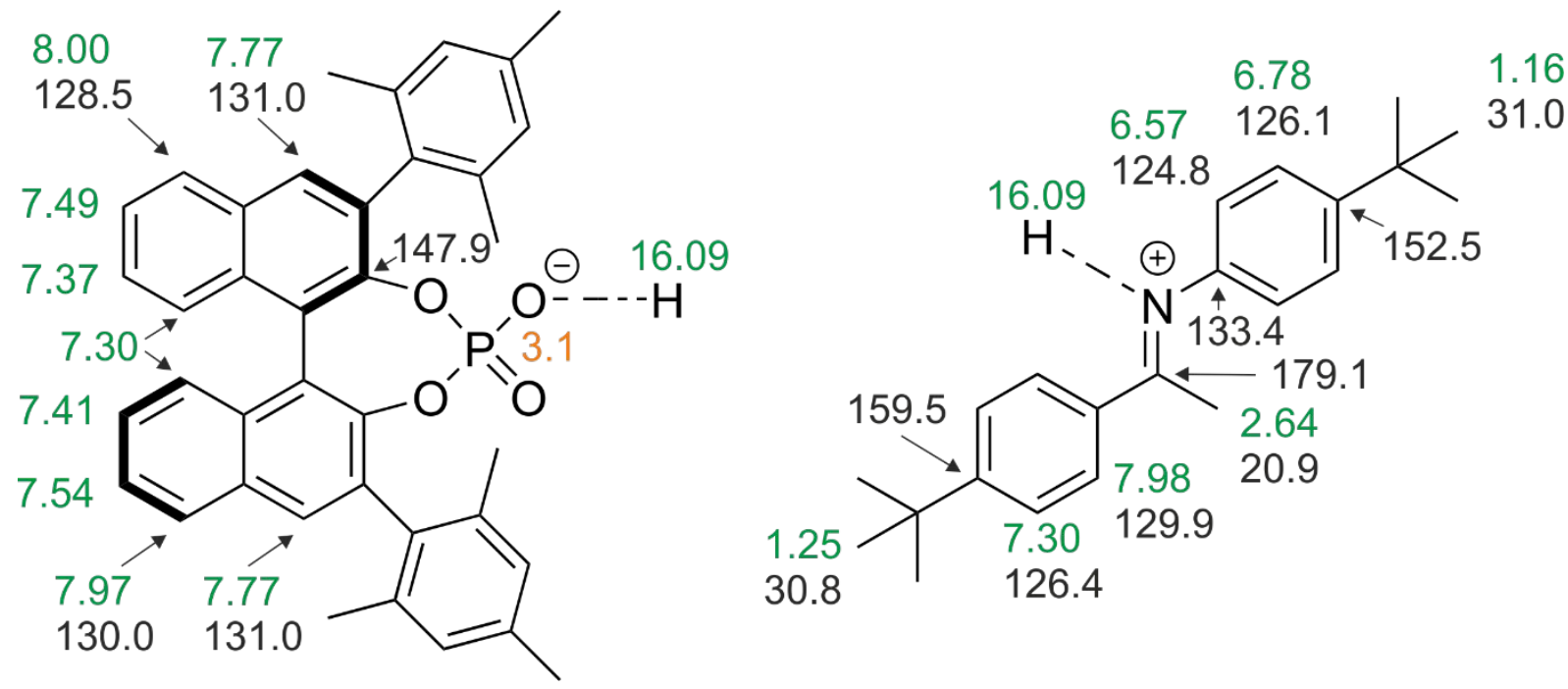

In accordance with our previous analysis of hydrogen bond strengths, ${ }^{1}$ the ${ }^{1} \mathrm{H}$ chemical shift of the hydrogen bond strength reflects the $\mathrm{H}$-bond strength (in our system, higher chemical shifts correlate with stronger $\mathrm{H}$-bonds). Hence, the $\mathrm{H}$-bond strength of TRIM/4E (16.09 ppm) is in between TRIM/1E (16.26 ppm) and TRIM/3E (15.80 ppm). ${ }^{9}$

Identification of Type I E and Type // E

Analogous to our previous work, ${ }^{2,9}$ for the Type I $E$ structure, the tert-butyl group of the ketone part of the imine (marked in blue) is placed above the BINOL backbone. The blue NOE cross signals $A$ and $B$ to the Naphthyl backbone reveal the presence of the Type I $E$ structure (Figure 7 ) for TRIM/4E. The NOE cross signal $D$ (black) is a result of overlapping NOE signals between the tert-butyl group and the naphthyl backbone and the tert-butyl group and the attached 
phenyl ring (backbone: $8.00 \mathrm{ppm}$, phenyl ring of imine: $7.98 \mathrm{ppm}$ ). For the Type // E structure, the tert-butyl group of the aniline part of the imine is placed above the BINOL backbone and thus the red NOE cross signals A-D reveal the presence of the Type // E structure.

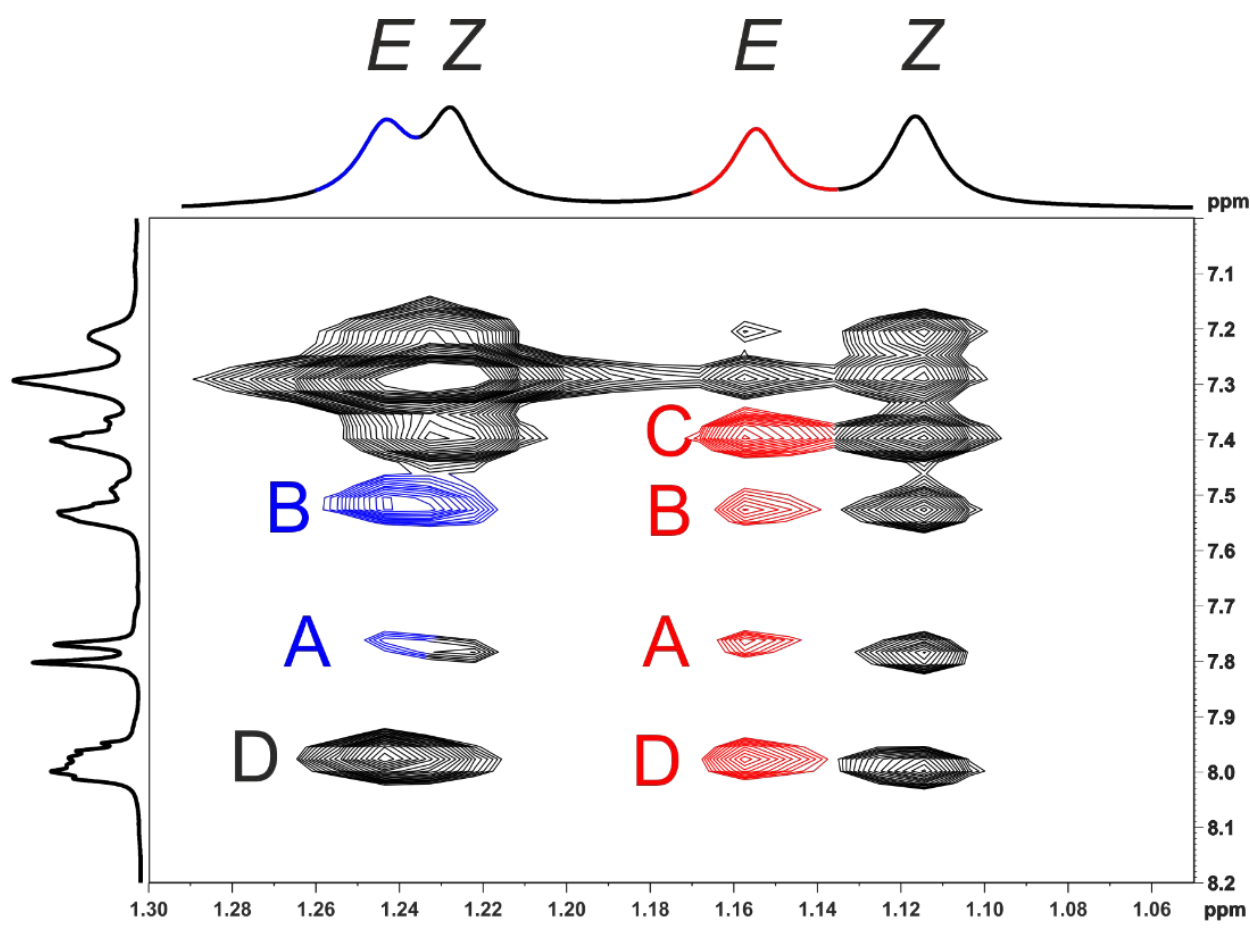

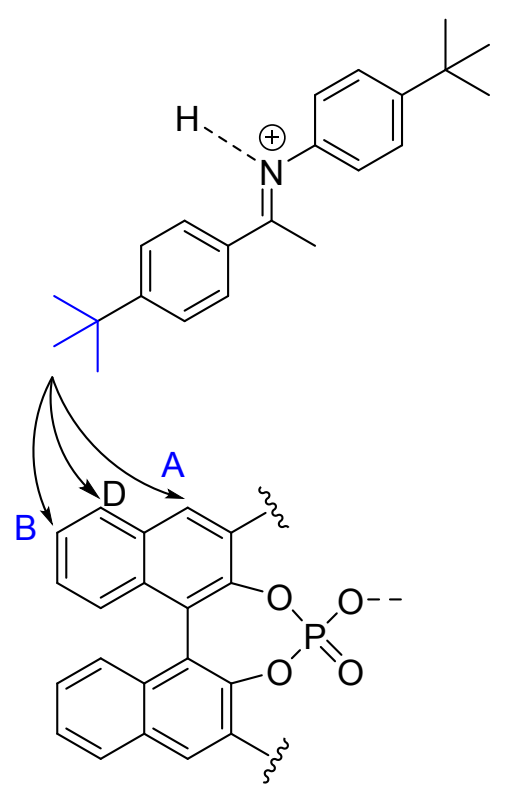

Type I E

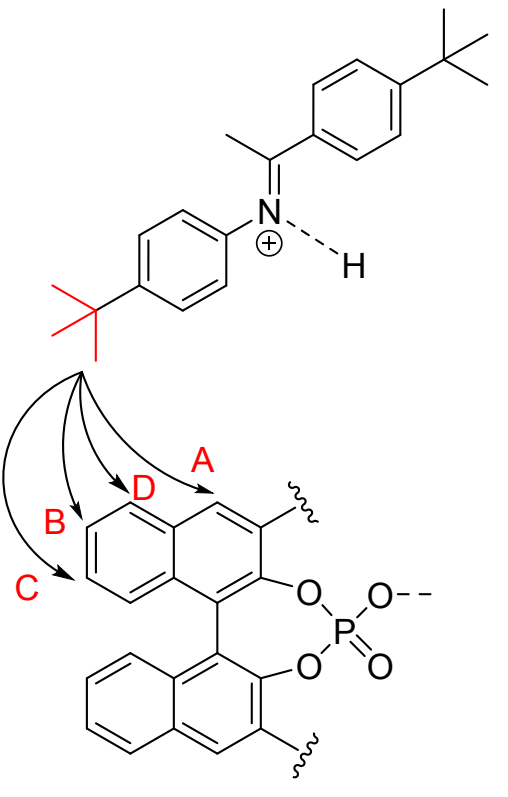

Type II E

Figure 7: Excerpt of the ${ }^{1} \mathrm{H},{ }^{1} \mathrm{H}$ NOESY spectrum of TRIM/4 at $180 \mathrm{~K}$ and $600 \mathrm{MHz}(100 \mathrm{mM}$ sample) in $\mathrm{CD}_{2} \mathrm{Cl}_{2}$. The blue NOE cross signals indicate the presence of the Type I E structure, while the red NOE signals indicate the presence of the Type // E structure. 
To prove, that the observed species in TRIM/4 are monomers of the binary complexes, DOSY measurements (Difusion ordered spectroscopy) were performed to derive the molecular radii of the present species.

The DOSY measurements were performed with the convection suppressing DSTE (double stimulated echo) pulse sequence developed by Jerschow and Müller in a pseudo 2D mode. ${ }^{10}$ TMS was used to reference the viscosity of the solvent at $180 \mathrm{~K}$. The diffusion time delay was set to $45 \mathrm{~ms}$. The gradient pulse lengths ( $\mathrm{p} 16$, SMSQ10.100 pulse shape) were optimized for each species to give a sigmoidal signal decay for varying gradient strengths. Optimal pulse lengths of $3.0 \mathrm{~ms}$ and $6.0 \mathrm{~ms}$ were found for TMS and TRIM/4, respectively. For each species, twenty spectra with linear varying gradient strength of $5 \%-95 \%$ have been measured. The used probe signals for the analysis are listed in table 1 . The signal intensities of the respective groups were analyzed as a function of the gradient strength by Bruker TopSpin 3.2 software T1/T2 relaxation package by employing the Stejskal-Tanner equation. ${ }^{11}$ No line broadening occurred for increasing gradient strength. The sigmoidal fit provided the translational selfdiffusion coefficients $D_{i}$ listed in Table 1 . The molecular radii were derived by the StokesEinstein equation ${ }^{12}$ using Chens correction. ${ }^{13}$

$$
D_{i}=\frac{k_{B} T}{6 \pi \eta r_{H}} *\left(1+0.695 *\left(\frac{r_{\text {solv }}}{r_{H}}\right)^{2.234}\right.
$$

$D_{i}$ is the self-diffusion coefficient derived by the measurement, $\eta$ is the viscosity of the solvent, $r_{H}$ is the hydrodynamic radius of the observed molecule and $r_{\text {solv }}$ the radius of the solvent. No form factor correction was applied. The viscosity was determined by measuring the diffusion coefficient of the reference tetramethylsilane (TMS) and solving the equation for $\eta$ with the literature value ${ }^{14}$ of the radius of $2.96 \AA$. The solvent radius of $\mathrm{CD}_{2} \mathrm{Cl}_{2}(2.46 \AA)$ was taken from the reference..$^{15}$

Table 1: Probe signals, measured diffusion coefficients and derived molecular radii for TRIM/5.

\begin{tabular}{|c|c|c|c|c|c|c|}
\hline Entry & Species & $\mathrm{p} 16[\mathrm{~ms}]$ & $\begin{array}{c}\text { Observed signal } \\
{[\mathrm{ppm}]}\end{array}$ & $\mathrm{D}_{\mathrm{i}}\left[\mathrm{m}^{2} / \mathrm{s}\right] \cdot 10^{-12}$ & Averaged & $\left.\mathrm{r}_{\mathrm{H}}[\AA]\right]$ \\
\hline 1 & TMS & 3.10 & 0.00 & 21.32 & & \\
\hline 2 & TRIM/4E & 6.00 & 1.16 & 4.76 & & \\
3 & TRIM/4E & 6.00 & 2.64 & 4.80 & 4.69 & 9.54 \\
4 & TRIM/4E & 6.00 & 7.77 & 4.50 & & \\
\hline
\end{tabular}

The derived molecular radius $(9.54 \AA)$ is similar to the one reported previously for TRIM monomers. ${ }^{9}$ Thus, the DOSY measurements showed, that the species investigated by the $R_{1 \rho}$ measurement is the binary TRIM/4E complex. 
A general rotational correlation time of $10-50$ ns was estimated for the investigated binary complexes of chiral phosphoric acids and imines with their hydration shell. Stokes Law (Eq. 5) was used to calculate the rotational correlation time based on the results of the DOSY measurements of TRIM/4E.

$$
\tau_{c}=\frac{4 \pi \eta r^{3}}{3 k T}
$$

The radius of the binary complex of TRIM/4 with its hydration shell was determined by DOSY measurements (see respective chapter) and a radius of $9.54 \AA$ was determined for the complex with its hydration shell. The viscosity $\left(3.05^{\star} 10^{-2} \mathrm{~kg} \mathrm{~m}^{-1} \mathrm{~s}^{-1}\right)$ of the solution at $180 \mathrm{~K}$ was determined by Equation 4 based on the DOSY measurement on TMS. A rotational correlation time of $44.6 \mathrm{~ns}$ was calculated for TRIM/4E with its hydration shell. Given the bulkiness of the two tert-butyl substituents of imine 4 , it is expected that the other investigated complexes have smaller radii, resulting in smaller rotational correlation times. Hence, a general rotational correlation time around $10-50$ ns was estimated. 
In the TiPSY/1 complex, the backbone splitting could not be resolved at $180 \mathrm{~K}$ and $600 \mathrm{MHz}$ in $\mathrm{CD}_{2} \mathrm{Cl}_{2}$ (see figure). However, in our previous investigations we observed, that the backbone splitting in TiPSY complexes is very small and could often not be resolved sufficiently (see SI of literature). ${ }^{9}$ Goodman et al. demonstrated, that the binding pocket for TiPSY is significantly smaller than for TRIP (no rotation possible). ${ }^{16}$ The biggest binding pocket for the investigated CPAs was determined for TRIM (rotation possible). Hence, a rotation of the imine inside the binding pocket of TiPSY can be excluded, even if the backbone splitting can not be resolved sufficiently.

The sample was prepared with an exclusive population of the E-imine. To achieve that, the sample was prepared according to the general procedure described above. However, before adding the solvent, the NMR-tube with TiPSY and 1 was cooled to $-90{ }^{\circ} \mathrm{C}$ in acetone/liquid $\mathrm{N}_{2}$ and the precooled solvend was added to the tube. The low temperature suppressed $E \rightarrow Z$ isomerization of the imine.

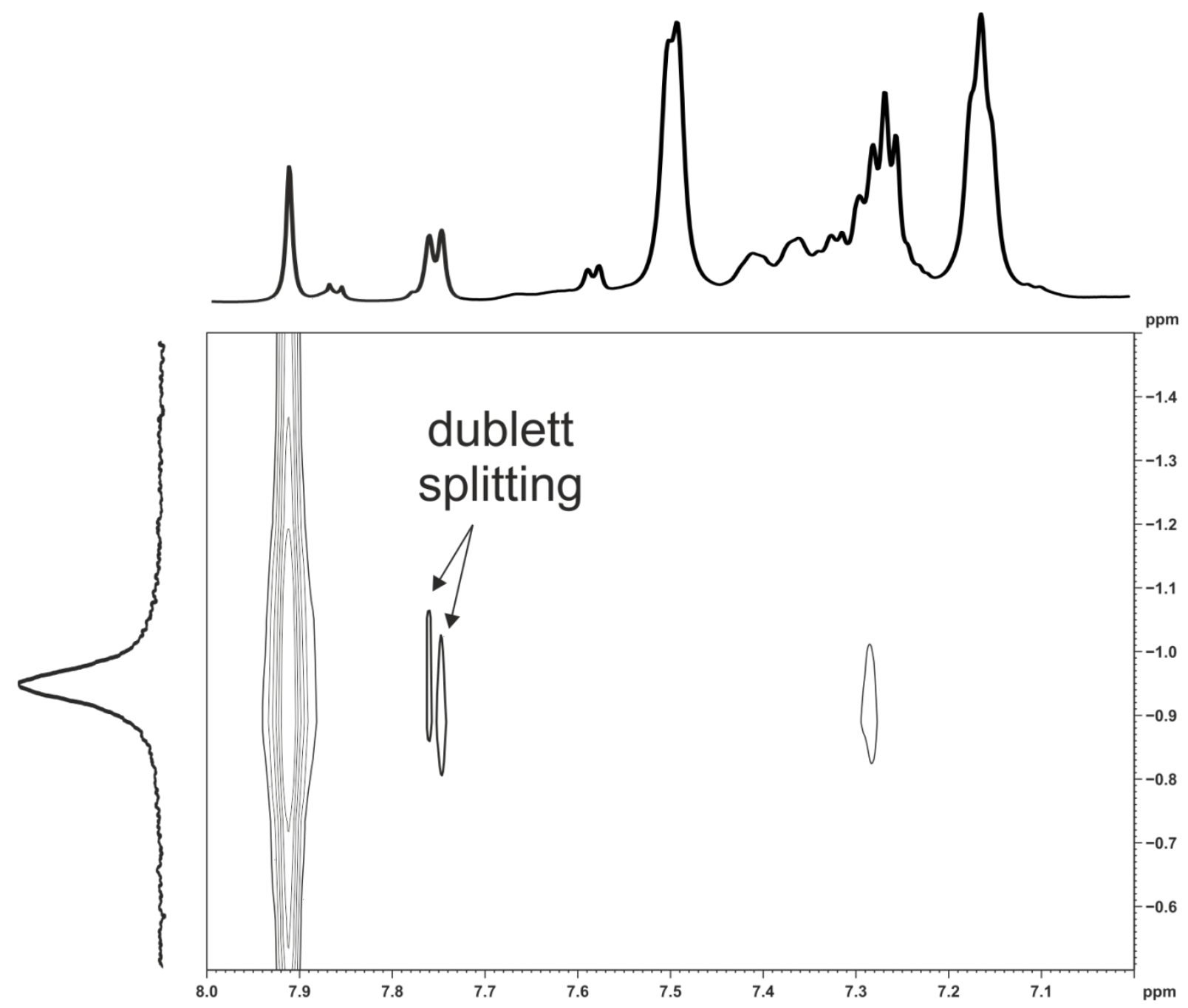

Figure 8: Excerpt of the ${ }^{1} \mathrm{H},{ }^{31} \mathrm{P}$ HMBC spectrum of TiPSY/1 (E-only, $25 \mathrm{mM}$, TiPSY:1 = 1:1) at $180 \mathrm{~K}$ and $600 \mathrm{MHz}$ in $\mathrm{CD}_{2} \mathrm{Cl}_{2}$. The sample was prepared with an exclusive population of $E$ imine species. 
Typically, in 1:1 complexes of TRIP, TRIM and TiPSY, the complexation of the imine was incomplete $(\approx 25 \%$ free imine present). Thus, for complexes with imines $1-3$, the assignment of the free imines was done for the CPA/imine samples. For imine 4, a NMR sample with the imine only ( $50 \mathrm{mM}, \mathrm{CD}_{2} \mathrm{Cl}_{2}, 180 \mathrm{~K}$ and $600 \mathrm{MHz}$ ) was measured and compared to the chemical shifts in the CPA/4 sample. For TRIM/4, no free imine was observed in the binary complex sample.

As the free imines 1-4 gave chemical shifts well separated from the respective CPA/E-imine complexes (see figure 9 for free imines and assignments of the binary complex in literature ${ }^{2,9}$ ), the exchange between free and complexated imine is slow on the NMR time scale. Thus, the exchange pathway via dissociation and re-association causing averaging of the BINOL backbone can be excluded to significantly contribute to the measured exchange rates in the $\mathrm{R}_{1 \sigma}$ measurements.
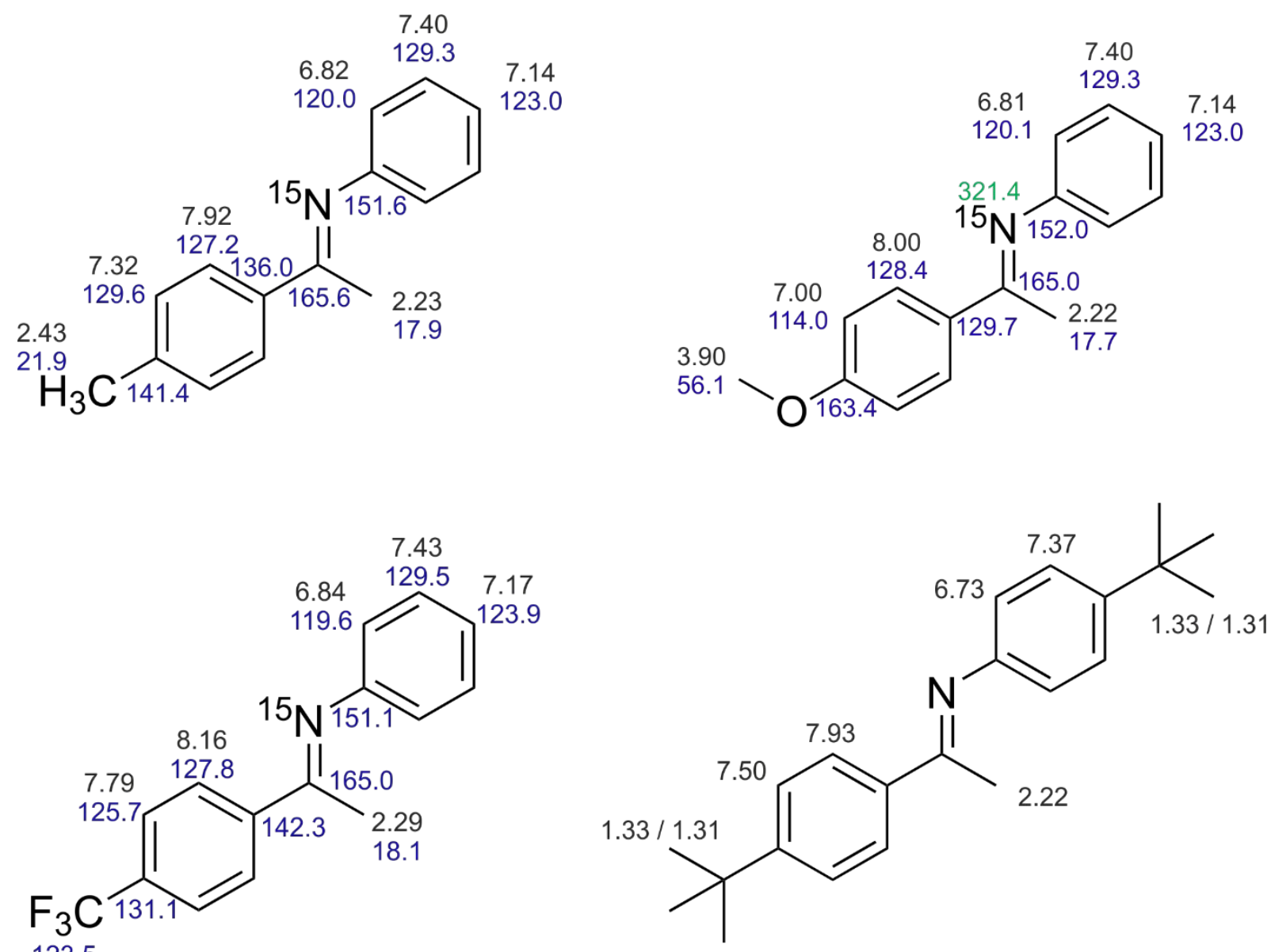

123.5

$-62.2$

Figure 9: Chemical shift assignment of the free imines 1-4 at $180 \mathrm{~K}$ in $\mathrm{CD}_{2} \mathrm{Cl}_{2}$. 


\section{Additional fitting of TRIM/1E}

Due to the strong signal decay within the first few percent of the horizontal axis for TRIM/1E (Figure 6B in the manuscript), fewer data points are in the decay region relevant for the fit, which could affect the quality of the fit and thus the precision of the extracted rate. However, the fit curves for lower $\left(500 \mathrm{~s}^{-1}\right)$ and higher $\left(10000 \mathrm{~s}^{-1}\right)$ exchange rates as the optimized one $\left(2500 \mathrm{~s}^{-1}\right)$ significantly deviate from the data set (Figure 10).
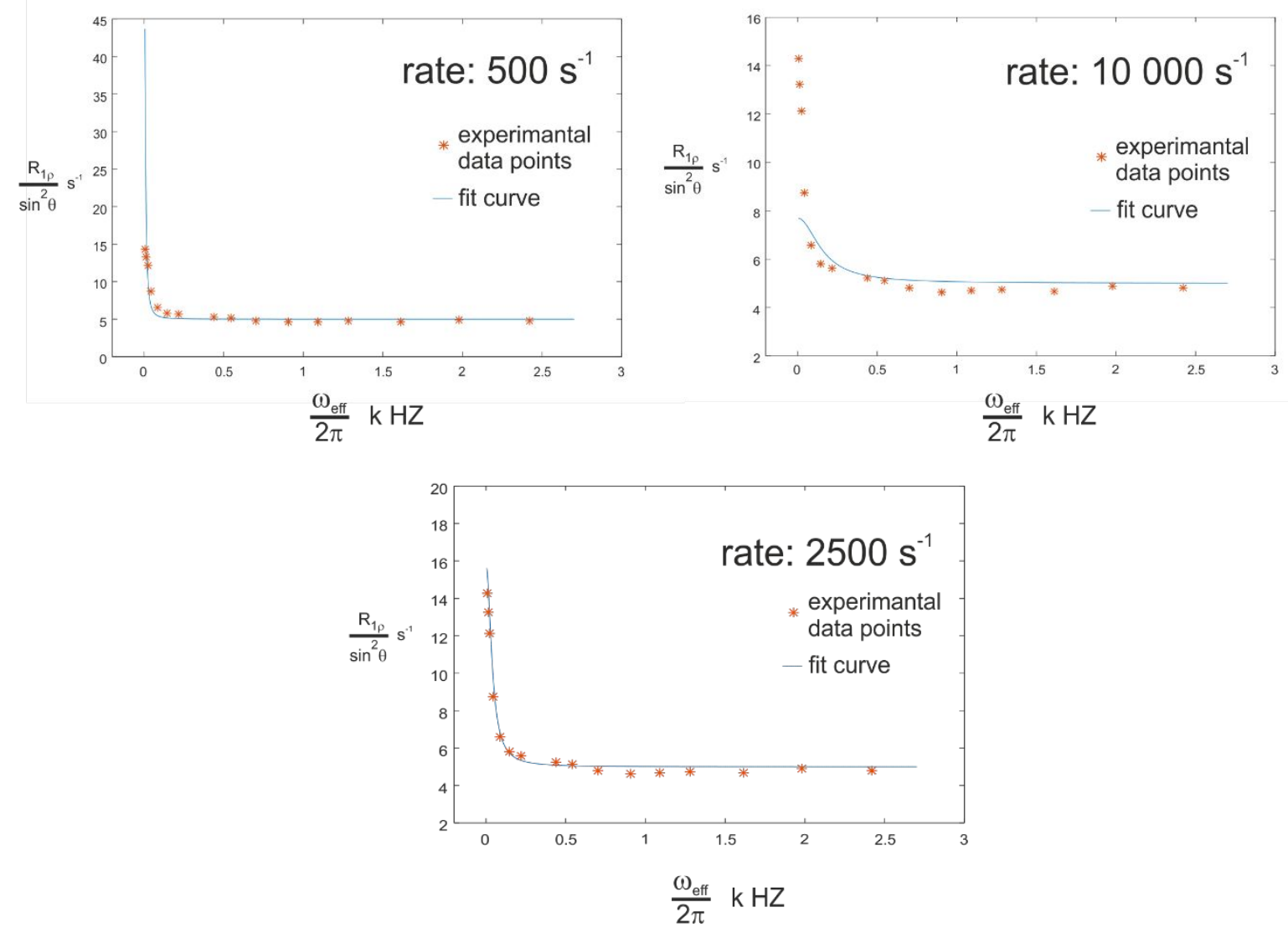

Figure 10: Additional curve fitting for the signal decay of TRIM/1E with different exchange rates at a concentration of $5 \mathrm{mM}$. The determined exchange rate of $2500 \mathrm{~s}^{-1}$ fits the data set best, while lower or higher exchange rates result in a significant offset. Other parameters, e.g. the population of $E-I$ and $E$-II were not changed.

In addition, a TRIM/1E sample at different concentration $(0.25 \mathrm{mM})$ was measured with more $\omega_{\text {eff }}$ increments. The exctracted exchange rate of $2500 \mathrm{~s}^{-1}$ is identical to the previous one and the fit for lower or higher exchange rates does not match the data set (Figure 11).

Hence, the quality of the fit for TRIM/1E is adequately precise to reveal, that the exchange rate for TRIM/1E is significantly lower than for TRIP/1E $\left(\approx 10000 \mathrm{~s}^{-1}\right)$. 

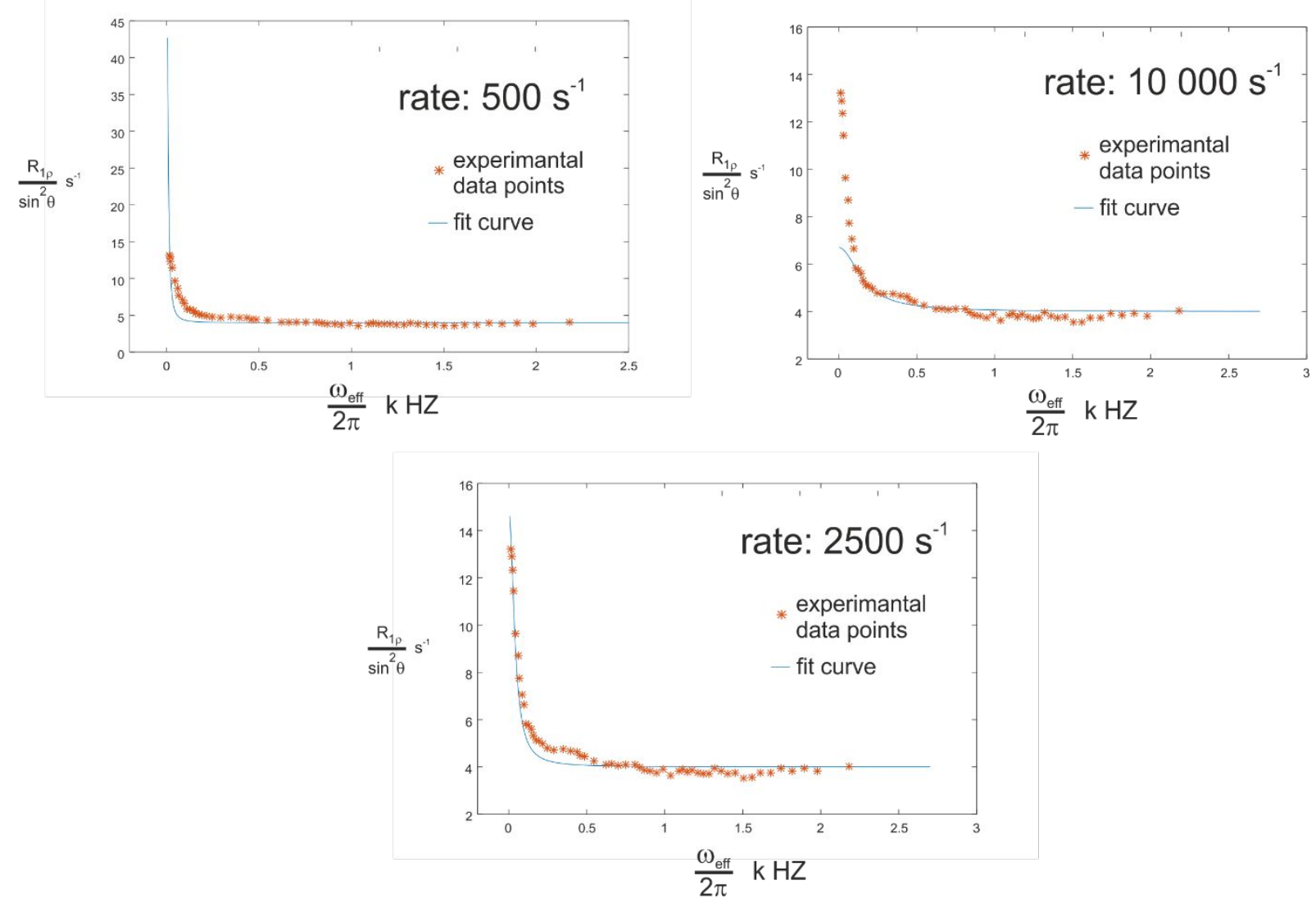

Figure 10: Additional curve fitting for the signal decay of TRIM/1E with different exchange rates at a concentration of $0.25 \mathrm{mM}$ and additional $\omega_{\text {eff }}$ increments. The determined exchange rate of $2500 \mathrm{~s}^{-1}$ fits the data set best, while lower or higher exchange rates result in a significant offset. Other parameters, e.g. the population of $E-I$ and $E$-II were not changed. 


\section{Computational Details}

All structures were optimized at TPSS/def2-SVP ${ }^{[17]}$ level of theory using $\mathrm{D}^{[18]}$ correction in continuum of $\mathrm{CH} 2 \mathrm{Cl} 2$ (SMD ${ }^{[19]}$ ). The dielectric constant was adapted to 16.20 to mimic low temperature condition in the experiment. Vibrational and thermochemical analyses were performed at the same level of theory as the geometry optimization. Subsequently, single point calculations at SCS-MP2/CBS[20] level of theory were conducted. Def2-SVP and def2-TZVP basis sets were used for extrapolation to approach complete basis set. Solvent and thermochemical corrections were added to the single point energy. Software used for the geometry optimization, frequency analysis and solvent correction was Gaussian 09 version D.01.[21] For single point calculation, ORCA 4.1.1 was used.[22] Constant temperature (NVT) molecular dynamic was done using semi-empirical xtb software in DCM. ${ }^{[23]}$

\section{Extrapolation procedure}

The CBS basis set extrapolation proceeds under the two point extrapolation procedure as in implemented in ORCA 4.1.1 with the basis set Def2-SVP and Def2-TZVP.

$E_{\mathrm{SCF}}^{X}=E_{\mathrm{SCF}}^{\infty}+A e^{-\alpha \sqrt{X}}$

$E_{\mathrm{corr}, \mathrm{MP} 2}^{\infty}=\frac{X^{\beta} E_{\mathrm{corr}, \mathrm{MP} 2}^{X}-(X-1)^{\beta} E_{\mathrm{corr}, \mathrm{MP} 2}^{X-1}}{X^{\beta}-(X-1)^{\beta}}$

$E_{\mathrm{MP} 2}^{\infty}=E_{\mathrm{corr}, \mathrm{MP} 2}^{\infty}+E_{\mathrm{SCF}}^{\infty}$

TRIP/1E Type III.1 and E-III.2 structures (most stable conformations)

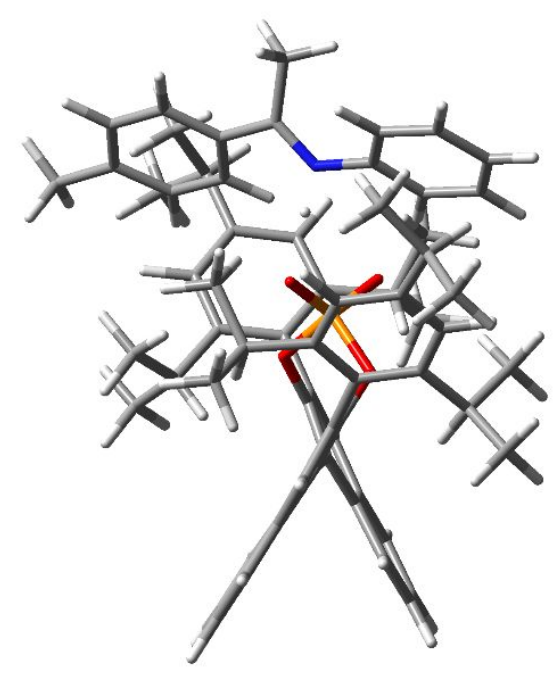

Type E-III.1

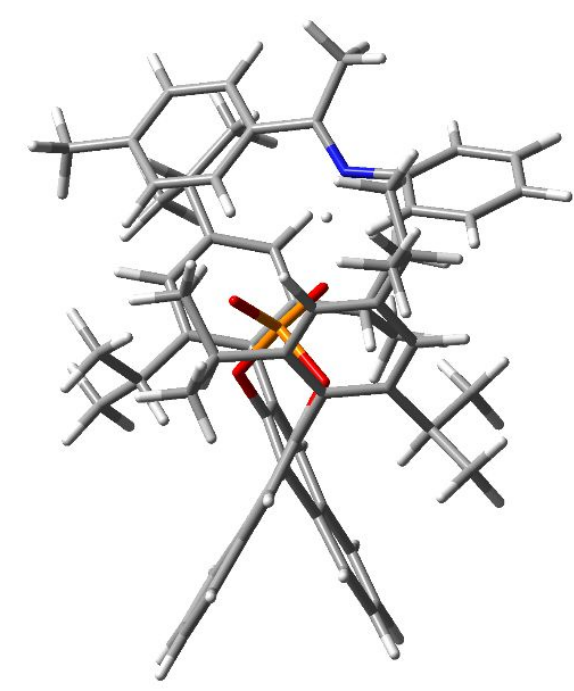

Type E-III.2 
MD of TRIP/1E complex shows the exchange of E-II and E-I conformations via tilting and switching. The E-II conformation is marked by the close contact between $\mathrm{C} 134$ and $\mathrm{H} 10$, while the $\mathrm{E}-\mathrm{I}$ conformation shows close contact between $\mathrm{C} 140$ and $\mathrm{H} 25$. No rotation is observed during a simulation of 4000 ps at $300 \mathrm{~K}$ to $370 \mathrm{~K}$, i.e. C134 never meets $\mathrm{H} 25$ or C140 never meets $\mathrm{H} 10$. Beyond this temperature, dissociation of complex is observed.

\section{Distance trajectory between atom C140 and atom H25}

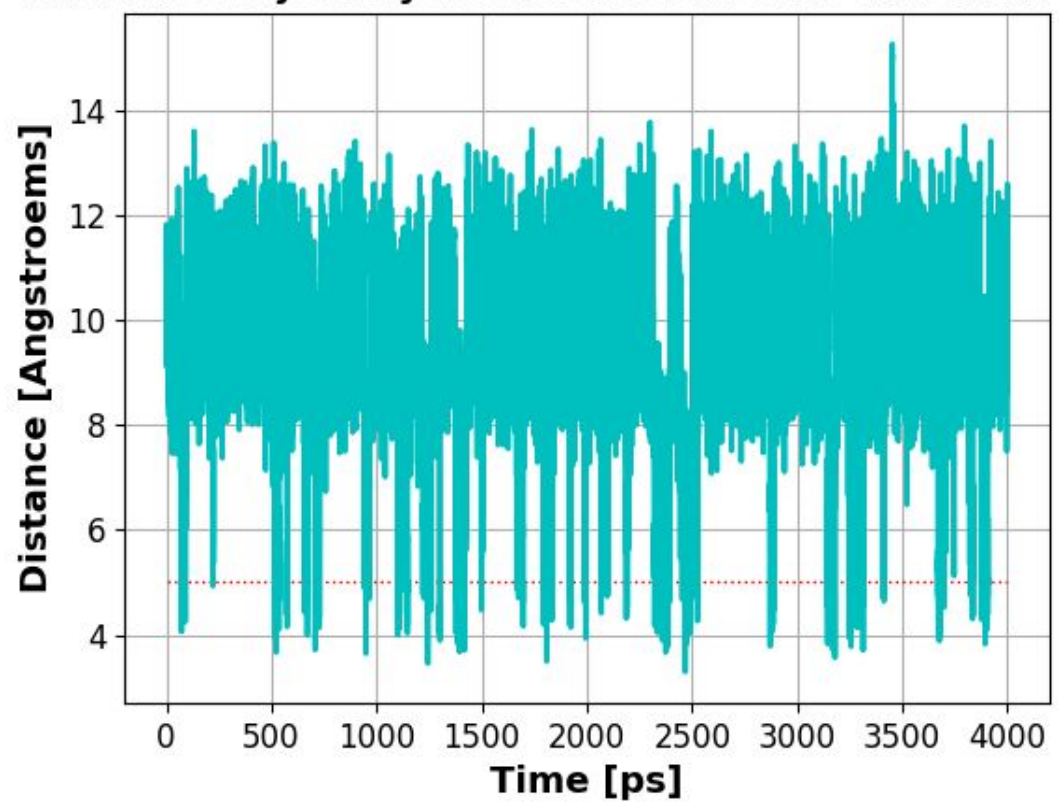

Distance trajectory between atom C140 and atom $\mathrm{H1O}$

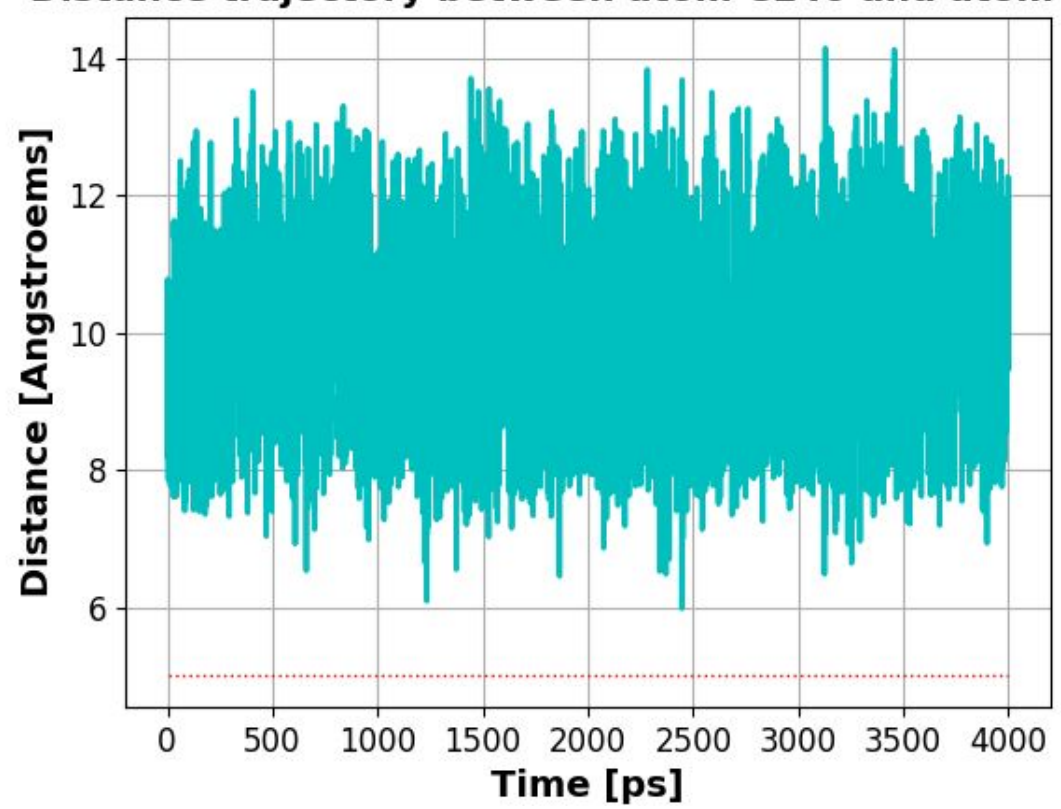




\section{Distance trajectory between atom C134 and atom H25}

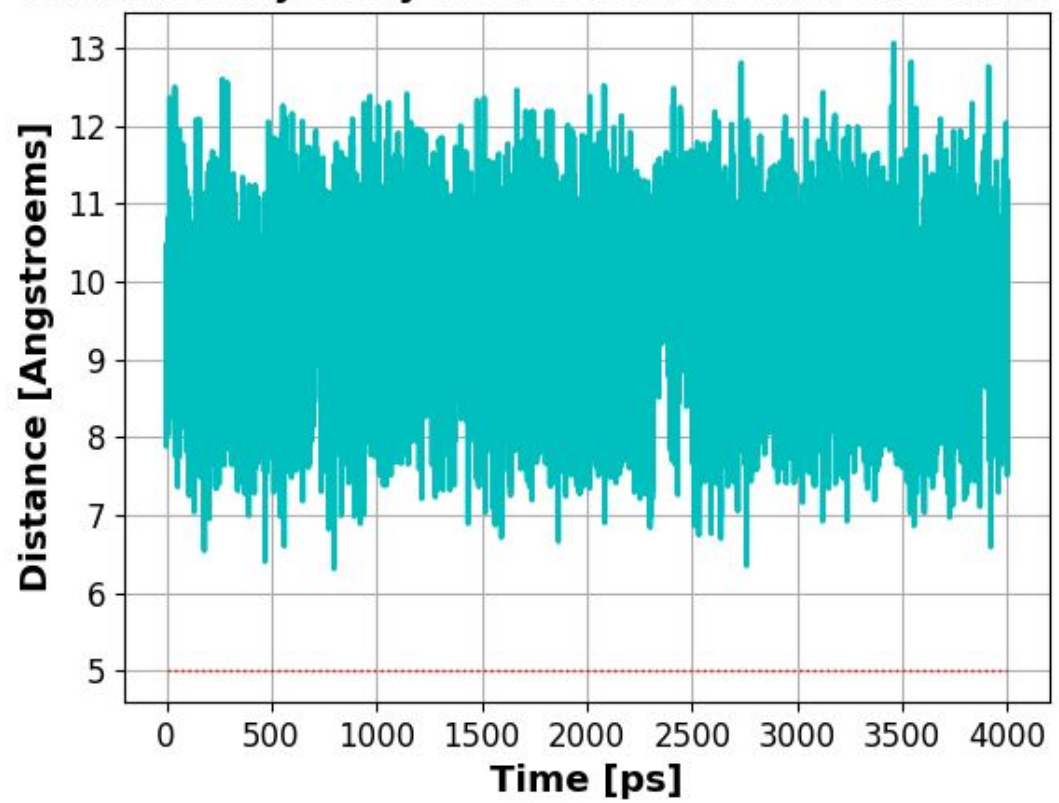

Distance trajectory between atom C134 and atom $\mathrm{H1O}$

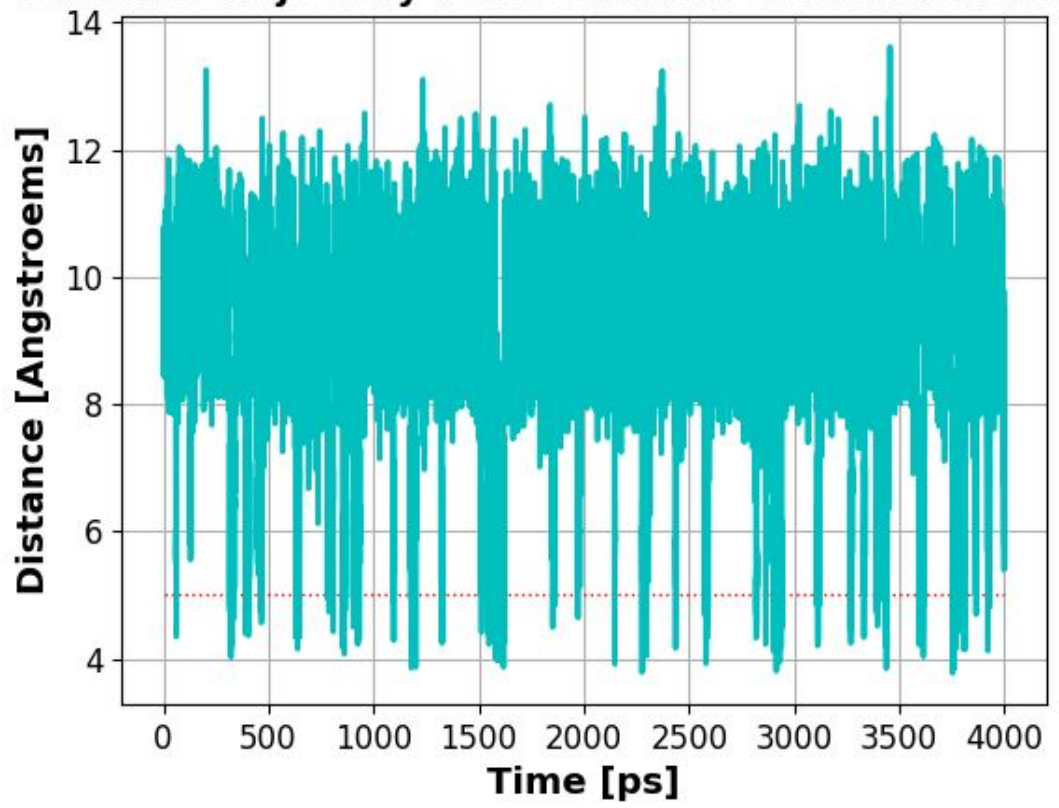

Optimized Geometries

TRIM/1E Type I

1।1\GINC-WORKER0IFOpt|RTPSSTPSSIdef2SVPIC53H48N1O4P1\JHIOE\24-Apr-2018

IO\॥p tpsstpss/def2svp empiricaldispersion=gd3 int=ultrafine opt scrf

$=($ smd, solvent $=$ ch2cl 2 , read) $)|t i t l e l| 0,1 \backslash P,-0.1297143417,-0.4860433703$,-

$1.1839383304 \backslash \mathrm{O},-1.4175075558,0.5618941922,-1.4320413577 \backslash 0,0.6889832102$

$, 0.3763631976,0.0168282895 \backslash 0,0.6300817555,-0.5399021282,-2.4784978792 \backslash$

O $-0.5766080585,-1.7420153175,-0.4359277394 \mathrm{IC},-1.446100498,2.147809437$

$, 0.3711360845 \backslash C,-2.0698317941,1.122837238,-0.3502138217 \backslash C,-3.395018365$

$8,0.6663965667,-0.0681477603 \mid C,-4.0703770977,1.2454430496,0.9985929916$

$\mathrm{IH},-5.095082782,0.9299435094,1.2232029452 \backslash \mathrm{C},-3.4593497944,2.2287931273$

, $1.8282185621 \backslash \mathrm{C},-2.1205478813,2.6764874421,1.5309470667 \backslash \mathrm{C},-1.502671696$

$6,3.5956395341,2.4329780968 \mathrm{lH},-0.4706962922,3.9085577634,2.251357641 \backslash \mathrm{C}$ 
$,-2.1840971756,4.0819139807,3.5389827427 / \mathrm{H},-1.6850717191,4.7804794202$, 4.2194778391।C,-3.5190578898,3.6730083748,3.8040883989\H,-4.0466914589 ,4.0672317544,4.679316788IC,-4.1381357835,2.7585716694,2.9673759161\H, $-5.1579812303,2.4147168377,3.1741719223 \backslash \mathrm{C},-0.0995474827,2.6345132883,-$ $0.0476174044 \backslash C, 0.9455071297,1.7164195775,-0.1873343455 \backslash C, 2.2926672419$, $2.1001225065,-0.4723187204 \backslash \mathrm{C}, 2.558703778,3.4483819763,-0.6657499207 \mathrm{MH}$, $3.5849117489,3.7741013745,-0.8683863455 \backslash C, 1.5225638135,4.4269654309,-0$ $.627942795 \mathrm{IC}, 0.1688315104,4.0227443182,-0.3306877178 \backslash \mathrm{C},-0.8544115593,5$ $.0185025682,-0.3633523236 \mathrm{H},-1.891720726,4.7236493662,-0.1814759087 \backslash \mathrm{C}$, $-0.5547814947,6.3456273979,-0.6331615051 \backslash \mathrm{H},-1.3588983494,7.0894174279$, $-0.6571408924 \backslash \mathrm{C}, 0.7839495245,6.7474855465,-0.8872017123 \backslash \mathrm{H}, 1.0082027204$ ,7.7998634367,-1.092917887\C,1.798284324,5.8032516275,-0.889462277\لH,2 $.8323097674,6.0967771622,-1.1038106254 \mid C,-0.8573168791,-0.0871264197,4$ $.830477067 \backslash \mathrm{C}, 0.3250076425,-0.6640802772,5.339846067 \backslash \mathrm{H}, 0.5983745847,-0$. $4987194234,6.3877175012 \mathrm{lC}, 1.1592184004,-1.4389797141,4.531247063 \mathrm{lH}, 2.0$ $63978865,-1.8641784067,4.9725342634 \mid C, 0.8369510599,-1.6781246632,3.170$ $7619493 \mid \mathrm{C},-0.3607975558,-1.1104431398,2.6642891292 \mathrm{H},-0.6471200511,-1$. $2563078529,1.6193677373 \mathrm{IC},-1.1818764103,-0.3336673895,3.4772889235 \mathrm{IH},-$ $2.0910080112,0.1022022382,3.050863107 \backslash \mathrm{C}, 1.7346779162,-2.4877720368,2.3$ $367466851 \backslash \mathrm{C}, 3.0307061219,-2.9840704286,2.9092756852 \mathrm{H}, 3.7204914907,-3$. $3197061151,2.1236646323 \mathrm{H}, 2.8474153916,-3.8258644017,3.6006465217 \backslash \mathrm{H}, 3$. $510628279,-2.179611337,3.4868696705 \backslash N, 1.4180260578,-2.7627175612,1.084$ $0668107 \backslash \mathrm{H}, 0.5664142926,-2.31860371,0.6154507535 \backslash \mathrm{C}, 2.1389962502,-3.6123$ $512175,0.1823560853 \backslash \mathrm{C}, 2.4359775179,-3.1249009192,-1.1037939824 \backslash \mathrm{H}, 2.142$ $6574357,-2.1148435791,-1.4029337988$ IC, $3.1228698719,-3.9483988815,-2.00$ $52601442 \backslash \mathrm{H}, 3.3672902628,-3.5612773484,-2.9999435028 \mathrm{lC}, 3.4986477652,-5$. 2502233614,-1.638539936IC,3.173288782,-5.7378013746,-0.3624422118।H,3. $4445753358,-6.7593282506,-0.0759896243 \backslash \mathrm{C}, 2.488803112,-4.9255523785,0.5$ $51014429 \mathrm{IH}, 2.2105874485,-5.3112801927,1.535916618 \mathrm{IC}, 3.3574813722,1.049$ $6078528,-0.5221976421 \backslash \mathrm{C}, 3.8820766371,0.6171273141,-1.7648178795 \backslash \mathrm{C}, 3.81$ $33037978,0.4648578409,0.6883645237 \backslash \mathrm{C}, 4.8433702185,-0.4131956559,-1.774$ 0880534IC, $4.7797271731,-0.5540322703,0.6330473451 \backslash \mathrm{C}, 5.3007424794,-1.01$ $73989861,-0.5905585388 \mathrm{H}, 5.2323858632,-0.7629238558,-2.7384472369 \mathrm{H}, 5$. $1287889563,-1.0034495867,1.5706543669$ IC $,-4.0422033474,-0.3715952492,-0$ $.93176383 \backslash \mathrm{C},-4.2439433849,-1.6877463165,-0.4441989481 \backslash \mathrm{C},-4.4757656684$, $-0.0220040175,-2.23750141 \backslash \mathrm{C},-4.8877849299,-2.6286176159,-1.2703109744 \backslash$ C,-5.1126323678,-0.9944206736,-3.0292151972lC,-5.3318232431,-2.3046804 $372,-2.5644701541 \mathrm{lH},-5.0403127902,-3.6466576615,-0.8904703152 \mathrm{H},-5.451$ $247875,-0.7173306478,-4.035527591 \mathrm{H}, 4.0355422138,-5.8879162062,-2.3487$ 788629lC,-1.7348499764,0.7905369911,5.6834699971। $\mathrm{H},-2.803088998,0.5594$ $643122,5.5276926166 \mathrm{H},-1.4973498997,0.687292941,6.7549605809 \mathrm{lH},-1.5936$ $724284,1.8509620385,5.40062661691 \mathrm{C},-4.2514305334,1.372187681,-2.780680$ 954) $\mathrm{H},-3.1760422211,1.5471962801,-2.9686362199 \mathrm{IC},-3.7234763064,-2.1051$ $234165,0.9133792143 \backslash \mathrm{C},-5.9947651326,-3.3402812992,-3.4460684157 \backslash \mathrm{H},-6.4$ $26078201,-4.1627788767,-2.849975659 \mid C, 6.2928701293,-2.1588417764,-0.62$ 10228897\H,6.6597440321,-2.3492514885,-1.6436977409IC,3.3756545,1.1994 $687316,-3.065533961 \backslash \mathrm{H}, 3.522494415,2.2931117325,-3.1124531776 \backslash \mathrm{C}, 3.25277$ $58092,0.9160828036,2.0191222363 \mathrm{H}, 2.1855866063,0.6444731169,2.10728233$ $77 \mathrm{H},-4.0946718188,-1.4538681333,1.7232781316 \mathrm{H},-4.7929250137,1.520997$ $9015,-3.7298269317 \mathrm{VH},-4.5831369161,2.146494748,-2.0656428727 \mathrm{H},-6.7978$ 06258,-2.8945925833,-4.0592879214l $,-5.2622090159,-3.7874543359,-4.145$ $8007235 \mathrm{IH},-4.0136105911,-3.1435800729,1.1457399925 \mathrm{H},-2.620832606,-2.0$ $443464438,0.9154533097 \backslash \mathrm{H}, 7.1653097059,-1.9530108279,0.0262097755 \backslash \mathrm{H}, 5.8$ $285797651,-3.0921608477,-0.2499486333 \backslash \mathrm{H}, 3.892931698,0.7461038806,-3.92$ $80518464 \mathrm{l}, 2.293546509,0.999192698,-3.1625821111 \mathrm{l}, 3.7944905462,0.4502$ $560301,2.8591103921 \mathrm{H}, 3.3149641322,2.013378366,2.1334561607 / I V e r s i o n=E$ S64L-G09RevD.01IState $=1-A \mid H F=-2744.5738521 / R M S D=5.533 e-09 / R M S F=4.297 \mathrm{e}-$ 06IDipole=1.9357807,-1.8507178,5.1028764 $\backslash$ Quadrupole $=-5.3970991,8.00075$ 3,-2.6036539,-20.8029182,2.3839022,-6.5868824IPG=C01 [X(C53H48N1O4P1)] $11 @$

\section{TRIM/1E Type II}

1/1IGINC-WORKEROIFOptIRTPSSTPSSIdef2SVPIC53H48N1O4P1\JHIOEI19-Feb-2019

IOI॥p tpsstpss/def2svp empiricaldispersion=gd3 int=ultrafine opt scrf $=(\mathrm{smd}$, solvent $=\mathrm{ch} 2 \mathrm{cl} 2$, read $) \|$ titlell0,1|P,0.2435317475,-0.5269533003,-1 .3378371932।O,-1.3323487876,-0.1477418071,-1.7685757054\O,0.4699021972 $, 0.6376544369,-0.11700720910,1.0960184283,-0.2617512622,-2.5438422821 \backslash$ $0,0.2959534707,-1.8258336027,-0.5385430013 \mathrm{IC},-2.2746832892,1.226493583$ $5,-0.047677137 \backslash \mathrm{C},-2.2773858888,0.0321480603,-0.7756252488 \backslash \mathrm{C},-3.2471058$ $074,-0.9997039478,-0.5809999831 \backslash \mathrm{C},-4.2152945273,-0.8056545488,0.395002$ $1261 \backslash \mathrm{H},-4.9823699696,-1.5717223468,0.5524632722 \backslash \mathrm{C},-4.2209376288,0.3501$ $188667,1.2262881953 \backslash \mathrm{C},-3.225216969,1.3750918933,1.0268646148 \mathrm{IC},-3.1934$ $6662,2.4692479859,1.9443982728 \mathrm{H},-2.4173230832,3.2323805375,1.83842853$ $67 \mathrm{C},-4.1146485351,2.5660998959,2.9772334299 \mathrm{H},-4.0613104839,3.4096361$ $667,3.6742146243 \backslash \mathrm{C},-5.121032943,1.5768782932,3.1444291675 \backslash \mathrm{H},-5.8471699$ 
$375,1.6686300398,3.959406921 \backslash \mathrm{C},-5.1677743596,0.490454057,2.2856082821 \backslash$ $\mathrm{H},-5.9232767536,-0.2925053324,2.4172527029 \mathrm{lC},-1.261439767,2.2777622615$ $,-0.3533282494|C, 0.0983997351,1.9471696664,-0.3419123043| C, 1.137467880$ $5,2.9186467402,-0.4742520895 \backslash C, 0.7708376939,4.2406915359,-0.688774965 \backslash$ $\mathrm{H}, 1.5474775973,5.008387768,-0.7766670916 \mathrm{IC},-0.5947547522,4.6274142175$, $-0.8167345806 \mid C,-1.6319984655,3.6360492501,-0.661695962 \backslash C,-2.988067127$ 4,4.0426678655,-0.8498283197\H,-3.7833449153,3.2953549778,-0.775682668 IC, $-3.3065011483,5.3611282579,-1.1392614848 \mathrm{IH},-4.354007383,5.646787500$ $3,-1.2860433405 \backslash \mathrm{C},-2.2857042932,6.3426535443,-1.2564074654 \backslash \mathrm{H},-2.551165$ $2176,7.3816816952,-1.4801307245 \backslash C,-0.957496745,5.9783557503,-1.1027315$ $506 \mathrm{H},-0.1597292807,6.7224823159,-1.2082502562 \backslash \mathrm{C}, 5.4833263034,-2.14012$ $29787,-1.1443327532 \mathrm{lC}, 5.7306421425,-2.8473179851,0.0522098605 \mathrm{H}, 6.6365$ $427024,-3.4572612938,0.140047481 \mathrm{lC}, 4.838970088,-2.7893630703,1.1275472$ 916।H,5.0525639248,-3.3668641124,2.0319307739IC,3.6499755476,-2.027028 $9312,1.0291316133 \backslash \mathrm{C}, 3.4173557768,-1.2912790454,-0.1566097527 \backslash \mathrm{H}, 2.56956$ $08405,-0.6079006844,-0.2168799366 \backslash \mathrm{C}, 4.311356835,-1.35684245,-1.2226122$ $605 \backslash \mathrm{H}, 4.096493846,-0.775685716,-2.1241443928 \mathrm{IC}, 2.6888004543,-2.0092236$ $362,2.1414654481 \mathrm{IC}, 3.1842586652,-2.1621662917,3.5478839498 \mathrm{H}, 2.4700187$ $721,-1.7501644495,4.2766823964 \mathrm{H}, 4.1573633458,-1.6581790304,3.65337519$ $65 \mathrm{IH}, 3.333581244,-3.2323566559,3.7814766065 \mathrm{WN}, 1.4108029046,-1.85027446$ $87,1.8706826158 \mathrm{H}, 1.0758599268,-1.7875177642,0.8447980496 \mathrm{lC}, 0.31534386$ $57,-1.8382452193,2.7946267404 \backslash \mathrm{C},-0.6331019434,-0.8060578198,2.67253913$ $84 \mathrm{IH},-0.4958150426,-0.0350402665,1.9100882794 \mathrm{CC},-1.7429072914,-0.79154$ $79705,3.5266631533 \mathrm{lH},-2.4739474544,0.0180946614,3.443952387 \backslash \mathrm{C},-1.92373$ $29882,-1.8128813717,4.472948404 \backslash \mathrm{C},-0.9900559545,-2.8571649464,4.564082$ $8742 \backslash \mathrm{H},-1.1370215966,-3.6679141932,5.2853058165 \backslash \mathrm{C}, 0.1329950313,-2.8770$ $53562,3.7255947437 \backslash \mathrm{H}, 0.8457036435,-3.7048113935,3.7730874932 \backslash \mathrm{C}, 2.57217$ $12299,2.5107711645,-0.3407121448 \mid C, 3.401556444,2.4279319575,-1.4861961$ 731\C,3.1002132823,2.2187172891,0.9446881804\C,4.7534919051,2.06716884 $75,-1.3224768813 \backslash \mathrm{C}, 4.4548225242,1.8624976753,1.0613766709 \backslash \mathrm{C}, 5.30138742$ $58,1.7820671853,-0.0602565273 \backslash \mathrm{H}, 5.390347601,1.9914610335,-2.2125268322$ $\mathrm{IH}, 4.8578875781,1.6338434849,2.0560987318 \backslash \mathrm{C},-3.2160576671,-2.243669579$ $2,-1.4118260971 \backslash \mathrm{C},-2.8299770476,-3.4773063639,-0.8233985007 \backslash \mathrm{C},-3.58450$ $36268,-2.1906600723,-2.7793020362 \mid C,-2.8242400561,-4.6365252919,-1.618$ $9340461 \backslash \mathrm{C},-3.5655293993,-3.3770693425,-3.538025964 \backslash \mathrm{C},-3.1905104272,-4$. $61074573,-2.9786059461 \mathrm{H},-2.5154407823,-5.5866750662,-1.1647402013 \mathrm{H},-$ $3.8534471233,-3.3321384655,-4.5958293755 \mathrm{lH},-2.7990717664,-1.8001211591$ ,5.1309162534IC,6.4543043622,-2.1752093848,-2.2972938392।H,6.901453801 $1,-1.1750217474,-2.4521108064 \mathrm{H}, 7.2711211387,-2.894557594,-2.123260083$ 3\H, 5.9401721209,-2.4446147531,-3.23762474\C,-3.9858706027,-0.88263478 $51,-3.4247287779 \backslash \mathrm{H},-3.1162056716,-0.2047077481,-3.5027870211 \backslash \mathrm{C},-2.3845$ $588995,-3.5414921068,0.6214908212 \mathrm{IC},-3.1797698382,-5.879398954,-3.8032$ $646024 \backslash \mathrm{H},-2.1963757235,-6.381849568,-3.7499755494 \backslash \mathrm{C}, 6.7409265654,1.345$ $3871767,0.0926836608 \backslash \mathrm{H}, 7.3105145598,1.4908840155,-0.8407242904 \mathrm{IC}, 2.832$ $8134937,2.65292794,-2.8697324635 \mathrm{H}, 2.3706978268,3.65082819,-2.97022757$ 25।C, $2.2176821247,2.268198748,2.1723583253 \mathrm{lH}, 1.4473667371,1.4768073419$ ,2.131711857\H,-3.2056148452,-3.2997725502,1.3196980058/H,7.2489790231 $, 1.9031614041,0.9002481813 \backslash \mathrm{H}, 6.7950936194,0.2723080333,0.3578110273 \mathrm{lH}$, $2.8074195659,2.1214195987,3.0927319637 \backslash \mathrm{H}, 1.6829617781,3.2318586091,2.2$ $518107812 \mathrm{H}, 3.6170677866,2.5576748417,-3.6397544227 \mathrm{H}, 2.0485128537,1.9$ $003627647,-3.0725255495 \mathrm{lH},-3.404337333,-5.674878539,-4.8637034339 \mathrm{H},-3$ $.9290812658,-6.6032508668,-3.4304449221 \mathrm{H},-4.3855651684,-1.0454863959$, $-4.4397324074 \mathrm{|H},-4.7538916732,-0.3559334004,-2.8298514312 \backslash \mathrm{H},-2.0092729$ $934,-4.5475773895,0.8738884663 \mathrm{H},-1.5760020982,-2.812302817,0.79703416$ 08IIVersion=ES64L-G09RevD.01।State=1-AlHF=-2744.5726292\RMSD=2.951e-09 IRMSF=2.887e-06IDipole=2.4766682,-1.5582316,4.8253892 $\backslash$ Quadrupole $=14.52$ $75706,-5.9773321,-8.5502385,-18.0566483,2.3442691,-15.847193 \mid P G=C 01[X$ (C53H48N1O4P1)]॥@

\section{TRIM/1E Switching TS}

1\1|GINC-LOGINISPIRTPSSTPSSIdef2SVPIC53H48N1O4P1\JHIOEI29-Mar-201910। \#p tpsstpss/def2svp int=ultrafine empiricaldispersion=gd3 scrf=(smd,so Ivent=ch2cl2, read,externaliteration, dovacuum) \titlell0,1|P,0,0.347012 $,-0.056312,-0.132915 \backslash 0,0,-0.8194,1.046381,0.395644 \backslash 0,0,-0.668745,-1.30$ $9898,-0.619532 \backslash 0,0,1.163112,-0.431289,1.083694 \backslash 0,0,1.052533,0.416998,-$ $1.389137 \backslash \mathrm{C}, 0,-2.946032,0.281851,-0.415818 \mathrm{IC}, 0,-1.949621,1.264503,-0.36$ $8658 \mid C, 0,-2.085861,2.528892,-1.022799 \backslash \mathrm{C}, 0,-3.222739,2.752256,-1.788624$ $\mathrm{IH}, 0,-3.354435,3.719799,-2.285454 \mid \mathrm{C}, 0,-4.22022,1.750685,-1.96306 \mathrm{IC}, 0,-$ $4.081593,0.488909,-1.278635 \backslash \mathrm{C}, 0,-5.068874,-0.516706,-1.512874 \backslash \mathrm{H}, 0,-4.9$ $5947,-1.493491,-1.033429 \mathrm{lC}, 0,-6.150728,-0.277409,-2.347088 \mathrm{H}, 0,-6.8912$ $62,-1.06728,-2.514845 \backslash \mathrm{C}, 0,-6.303621,0.979797,-2.991572 \mathrm{H}, 0,-7.166332,1$ $.158216,-3.642726 \mathrm{IC}, 0,-5.351757,1.969675,-2.805804 \mathrm{IH}, 0,-5.447543,2.937$ $579,-3.311058 \mid C, 0,-2.797116,-0.951281,0.40804 \backslash C, 0,-1.646416,-1.731182$, $0.262026 \mathrm{IC}, 0,-1.458557,-2.975663,0.939812 \backslash \mathrm{C}, 0,-2.457905,-3.401042,1.80$ $6001 \backslash \mathrm{H}, 0,-2.351361,-4.361161,2.322682 \backslash \mathrm{C}, 0,-3.616893,-2.613052,2.061077$ IC, $0,-3.789386,-1.358426,1.370237 \backslash \mathrm{C}, 0,-4.927034,-0.558948,1.697152 \backslash \mathrm{H}, 0$ 
$,-5.051044,0.414622,1.214188 \mid \mathrm{C}, 0,-5.863523,-0.992075,2.624085 \backslash \mathrm{H}, 0,-6.7$ $25085,-0.358479,2.862143 \backslash \mathrm{C}, 0,-5.712084,-2.246915,3.273633 \mathrm{lH}, 0,-6.46228$ 2,-2.580411,3.998989|C,0,-4.606352,-3.03591,2.999637\H,0,-4.465903,-3. $996337,3.508803 \backslash \mathrm{C}, 0,-0.237275,-3.803668,0.687657 \backslash \mathrm{C}, 0,0.776864,-3.90790$ $5,1.676037 \backslash \mathrm{C}, 0,-0.10901,-4.500115,-0.539959 \mathrm{IC}, 0,1.906551,-4.701811,1.4$ 08065IC, $0,1.030429,-5.297531,-0.757943 \backslash \mathrm{C}, 0,2.054769,-5.406285,0.198392$ $|\mathrm{H}, 0,2.698138,-4.765781,2.165529| \mathrm{H}, 0,1.11932,-5.84353,-1.705373 \mathrm{lC}, 0,-1$ $.063898,3.607726,-0.836329|\mathrm{C}, 0,-0.112245,3.883085,-1.850966| \mathrm{C}, 0,-1.083$ $45,4.378063,0.353216|\mathrm{C}, 0,0.807708,4.929139,-1.651564| \mathrm{C}, 0,-0.155075,5.4$ $25578,0.504643 \mathrm{IC}, 0,0.802564,5.715401,-0.4839851 \mathrm{H}, 0,1.552152,5.133096$,$2.431437 \mathrm{MH}, 0,-0.172304,6.0193,1.427014 \mathrm{IC}, 0,-2.070995,4.065463,1.455446$ $|\mathrm{H}, 0,-1.875115,3.063586,1.880803| \mathrm{C}, 0,-0.05916,3.039217,-3.104875 \mid \mathrm{C}, 0,1$ $.83792,6.798274,-0.276282 \backslash \mathrm{H}, 0,2.818032,6.353849,-0.015613 \backslash \mathrm{C}, 0,3.301895$ ,-6.216979,-0.075022\H,0,3.532767,-6.900737,0.761976lC $, 0,0.682375,-3.1$ $29948,2.969749 \mathrm{H}, 0,-0.229323,-3.382758,3.539211 \mathrm{lC}, 0,-1.167519,-4.37968$ 8,-1.613991।H, $,-1.166843,-3.361028,-2.043893 \mid \mathrm{H}, 0,-1.027942,3.036937,-$ 3.635568|C, $0,3.140834,3.620855,2.786681 \backslash \mathrm{C}, 0,4.329843,2.952414,3.148093$ $\mid \mathrm{H}, 0,4.8669,3.255577,4.053427 \backslash \mathrm{C}, 0,4.833401,1.903037,2.374798 \backslash \mathrm{H}, 0,5.747$ $57,1.398858,2.699477 \backslash \mathrm{C}, 0,4.152672,1.476197,1.208129 \backslash \mathrm{C}, 0,2.964809,2.159$ $26,0.836006 \mathrm{H}, 0,2.435748,1.894154,-0.085368 \mathrm{lC}, 0,2.47287,3.201691,1.614$ $503 \mathrm{lH}, 0,1.557825,3.712501,1.30136 \mathrm{IC}, 0,4.657005,0.372943,0.386862 \mathrm{lC}, 0,6$ $.091485,-0.046117,0.445774 \backslash \mathrm{H}, 0,6.418433,-0.091579,1.497112 \backslash \mathrm{H}, 0,6.72562$ $3,0.706387,-0.0566011 \mathrm{H}, 0,6.254765,-1.027074,-0.022288 \mathrm{IN}, 0,3.802075,-0$. $239926,-0.412088|\mathrm{H}, 0,2.790923,-0.020217,-0.295224| \mathrm{C}, 0,4.050581,-1.2045$ 42,-1.433453|C, $0,5.074223,-1.016606,-2.380772 \mathrm{lH}, 0,5.704745,-0.124159,-$ $2.340972 \mathrm{lC}, 0,5.24954,-1.966413,-3.395697 \mathrm{MH}, 0,6.042364,-1.822151,-4.136$ 967IC, $0,4.403256,-3.08369,-3.473159 \mid \mathrm{C}, 0,3.372155,-3.249814,-2.534329 \mathrm{IH}$ $, 0,2.705328,-4.114723,-2.59327 \mid \mathrm{C}, 0,3.186153,-2.312395,-1.51146 \mathrm{IH}, 0,2.3$ 92023,-2.44065,-0.7698921H,0,4.53996,-3.820831,-4.2713।C,0,2.592378,4. $765222,3.599978 \mid \mathrm{H}, 0,3.152732,4.907779,4.538175 \mathrm{H}, 0,2.639969,5.706864,3$ $020565 \backslash \mathrm{H}, 0,1.527809,4.596686,3.843477 \backslash \mathrm{H}, 0,1.555307,-3.330388,3.61393 \backslash$ $\mathrm{H}, 0,0.650908,-2.048068,2.74636 \mathrm{IH}, 0,3.200887,-6.816732,-0.995306 \mathrm{lH}, 0,4$. $179015,-5.55393,-0.200795 \mathrm{H}, 0,-2.003531,4.803716,2.272054 \mathrm{l} \mathrm{H}, 0,-3.11022$ $7,4.055715,1.079668 \backslash \mathrm{H}, 0,0.713565,3.409795,-3.799855 \mathrm{l}, 0,0.178815,1.992$ $656,-2.839105 \backslash \mathrm{H}, 0,1.989383,7.39648,-1.192536 \backslash \mathrm{H}, 0,1.553101,7.48075,0.54$ $2539 \mathrm{H}, 0,-0.987261,-5.09625,-2.432772 \backslash \mathrm{H}, 0,-2.180584,-4.558621,-1.21092$ $4|I V e r s i o n=E S 64 L-G 09 R e v D .01| S t a t e=1-A l H F=-2744.5365861 \mid R M S D=5.105 e-091$ Dipole $=4.9306913,0.7434514,0.6187754$ Quadrupole $=33.8890338,-12.5399608$ $,-21.349073,3.2576982,1.1289285,12.9675487 \mathrm{PG}=\mathrm{C} 01$ [X(C53H48N1O4P1)]1@

\section{TRIP/1E Type II}

\section{Confo}

111IGINC-WORKER0ISPIRTPSSTPSSIdef2SVPIC65H72N1O4P1 IJHIOEI05-May-201910

I॥p tpsstpss/def2svp int=ultrafine empiricaldispersion=gd3 scrf=(smd, solvent=ch2cl2, read,externaliteration, dovacuum) |title $\mid 10,1 \backslash \mathrm{P}, 0,-0.211$ $306,-0.543612,-1.0515810,0,-1.540091,0.439014,-1.32767610,0,0.72606,0$. $529948,-0.11835910,0,0.385891,-0.829223,-2.396274 \backslash 0,0,-0.547219,-1.626$ $422,-0.030592 \backslash C, 0,-1.478252,2.243368,0.238585 \backslash C, 0,-2.112545,1.104448,-$ $0.262652 \backslash C, 0,-3.368933,0.626001,0.22375 \backslash C, 0,-3.958837,1.315777,1.27395$ $4 \mathrm{IH}, 0,-4.934559,0.993605,1.651432 \backslash \mathrm{C}, 0,-3.316655,2.423498,1.898685 \mathrm{IC}, 0$ $-2.049237,2.891538,1.393179 \backslash \mathrm{C}, 0,-1.388242,3.943195,2.097643 \backslash \mathrm{H}, 0,-0.405$ $365,4.279643,1.755322 \mathrm{IC}, 0,-1.961277,4.523334,3.220465 \mathrm{IH}, 0,-1.428719,5$. $320343,3.750891 \backslash \mathrm{C}, 0,-3.227744,4.086329,3.693607 \backslash \mathrm{H}, 0,-3.671901,4.55561$, $4.578195 \backslash \mathrm{C}, 0,-3.887699,3.05469,3.044385 \mathrm{IH}, 0,-4.854138,2.691907,3.41240$ $1 \mathrm{IC}, 0,-0.212075,2.72164,-0.386198 \mid \mathrm{C}, 0,0.874696,1.847981,-0.499038 \mathrm{IC}, 0$, $2.180442,2.281763,-0.890914 \backslash \mathrm{C}, 0,2.339154,3.604938,-1.278893 \backslash \mathrm{H}, 0,3.3311$ $92,3.965002,-1.571016 \backslash \mathrm{C}, 0,1.239185,4.510508,-1.319892 \backslash \mathrm{C}, 0,-0.058969,4$. $071167,-0.868889 \mathrm{IC}, 0,-1.149475,4.989465,-0.949817 \backslash \mathrm{H}, 0,-2.147298,4.6607$ $72,-0.644502 \backslash \mathrm{C}, 0,-0.963586,6.28056,-1.421893 \mathrm{l}, 0,-1.817054,6.964956,-1$ $.481725 \mathrm{IC}, 0,0.322065,6.720762,-1.836525 \mathrm{IH}, 0,0.45617,7.74472,-2.202098 \mathrm{I}$ $\mathrm{C}, 0,1.398728,5.849143,-1.788784 \mathrm{H}, 0,2.392434,6.172002,-2.1198 \mathrm{IC}, 0,3.55$ $0461,-4.331604,-1.369587 \backslash \mathrm{C}, 0,3.504131,-5.127359,-0.204594 \backslash \mathrm{H}, 0,3.902079$ ,-6.147731,-0.231619।C,0,2.963616,-4.634861,0.986739\H,0,2.932985,-5.2 $77792,1.871777 \backslash \mathrm{C}, 0,2.4182,-3.33021,1.037007 \backslash \mathrm{C}, 0,2.478781,-2.522418,-0$. $122918 \mathrm{lH}, 0,2.154709,-1.479998,-0.082659 \mid \mathrm{C}, 0,3.033534,-3.020473,-1.3013$ $42 \backslash \mathrm{H}, 0,3.080199,-2.369575,-2.178535 \backslash \mathrm{C}, 0,1.830766,-2.824969,2.287924 \backslash \mathrm{C}$, $0,2.467037,-3.194444,3.593188 \mathrm{H}, 0,2.267833,-2.440306,4.370141 \backslash \mathrm{H}, 0,3.55$ $2801,-3.308125,3.453202 \mathrm{H}, 0,2.069053,-4.163289,3.948083 \backslash \mathrm{N}, 0,0.77992,-2$ $.037666,2.208018 \mathrm{H}, 0,0.33831,-1.849618,1.235057 \backslash \mathrm{C}, 0,0.061755,-1.39607$, 3.263479|C $, 0,-0.351154,-0.068852,3.034427 \backslash \mathrm{H}, 0,-0.097396,0.41785,2.0887$ $21 \backslash \mathrm{C}, 0,-1.077239,0.606113,4.022743 \backslash \mathrm{H}, 0,-1.383219,1.64216,3.851368 \mathrm{IC}, 0$, $-1.417362,-0.04301,5.220411 \backslash \mathrm{C}, 0,-1.033615,-1.377385,5.426056 \backslash \mathrm{H}, 0,-1.31$ $7798,-1.898061,6.346438 \backslash \mathrm{C}, 0,-0.295495,-2.062354,4.450965 \mathrm{H}, 0,-0.036356$ ,-3.113063,4.598809\C,0,3.336628,1.33234,-0.800761।C,0,3.915921,0.7755 $77,-1.968791 \backslash \mathrm{C}, 0,3.850003,0.993892,0.484423 \backslash \mathrm{C}, 0,5.003799,-0.110133,-1$. 
$828311 \backslash \mathrm{C}, 0,4.935727,0.107662,0.569663 \backslash \mathrm{C}, 0,5.525536,-0.462006,-0.574498$ $\mathrm{IH}, 0,5.452257,-0.550665,-2.726998 \mathrm{lH}, 0,5.333994,-0.149227,1.55639 \mathrm{IC}, 0,-$ $4.027711,-0.556554,-0.418635 \backslash C, 0,-4.140596,-1.788724,0.281067 \backslash C, 0,-4.5$ $56491,-0.436036,-1.73424 \backslash \mathrm{C}, 0,-4.820243,-2.856965,-0.336704 \backslash \mathrm{C}, 0,-5.2153$ $41,-1.536361,-2.309187 \backslash \mathrm{C}, 0,-5.368139,-2.755254,-1.625233 \backslash \mathrm{H}, 0,-4.919699$ $,-3.807524,0.200857 \backslash \mathrm{H}, 0,-5.62568,-1.434712,-3.320788 \mathrm{H}, 0,-1.9926,0.487$ $458,5.986459 \mathrm{IC}, 0,4.183327,-4.834289,-2.642924 \backslash \mathrm{H}, 0,5.148579,-4.32093,-2$ $.816551 \mathrm{H}, 0,4.377135,-5.91883,-2.603884 \mathrm{lH}, 0,3.544212,-4.618103,-3.5172$ $93 \backslash \mathrm{C}, 0,-4.446235,0.859632,-2.540603 \mathrm{H}, 0,-3.879719,1.589296,-1.938393 \backslash \mathrm{C}$ $, 0,-3.535912,-1.994504,1.672265 \backslash \mathrm{C}, 0,-6.101618,-3.933163,-2.261641 \backslash \mathrm{H}, 0$, $-6.057279,-4.770799,-1.540158 \mathrm{IC}, 0,-4.619082,-1.953399,2.770682 \backslash \mathrm{H}, 0,-4$. $161227,-2.066325,3.770652 \backslash \mathrm{H}, 0,-5.177649,-1.001479,2.757559 \mathrm{H}, 0,-5.3486$ $15,-2.774026,2.636064 \mathrm{IC}, 0,-5.416272,-4.40016,-3.562365 \mathrm{IH}, 0,-5.442756$,$3.609819,-4.33503 \backslash \mathrm{H}, 0,-4.358689,-4.664757,-3.384253 \backslash \mathrm{H}, 0,-5.928636,-5.2$ $89346,-3.973679 \mathrm{IC}, 0,-7.589252,-3.606155,-2.507415 \mathrm{IH}, 0,-8.120146,-4.486$ $754,-2.913639 \mathrm{H}, 0,-8.092262,-3.303874,-1.571371 \backslash \mathrm{H}, 0,-7.701305,-2.78035$ 7,-3.234143\C,0,-3.662705,0.645066,-3.851851\H,0,-4.198502,-0.038602,$4.536188 \backslash \mathrm{H}, 0,-3.525028,1.606874,-4.379728 \backslash \mathrm{H}, 0,-2.665932,0.220389,-3.64$ $439 \mathrm{IC}, 0,-5.835215,1.477034,-2.805138 \mathrm{H}, 0,-5.736264,2.43928,-3.340548 \mathrm{H}$ $, 0,-6.4627,0.809889,-3.424663 \mathrm{lH}, 0,-6.373232,1.666264,-1.858714 \mathrm{IC}, 0,6.7$ $07782,-1.420808,-0.461291 \backslash \mathrm{H}, 0,6.840794,-1.886749,-1.456082 \backslash \mathrm{C}, 0,3.40372$ $5,1.123251,-3.367957 \backslash \mathrm{H}, 0,2.4598,1.681642,-3.244981 \backslash \mathrm{C}, 0,3.281127,1.6374$ $82,1.751527 \mathrm{WH}, 0,2.220706,1.873564,1.558832 \backslash \mathrm{C}, 0,8.005338,-0.654974,-0.1$ $25241 \mathrm{lH}, 0,8.869421,-1.343745,-0.086424 \mathrm{H}, 0,8.218831,0.121627,-0.881304$ $\mathrm{IH}, 0,7.923498,-0.15651,0.858572 \mathrm{CC}, 0,6.453175,-2.55077,0.555718 \mathrm{H}, 0,6.3$ $7807,-2.158554,1.586606 \mathrm{H}, 0,5.51717,-3.089141,0.327836 \mathrm{H}, 0,7.282708,-3$ $.281056,0.538706 \mathrm{IC}, 0,3.319578,0.719135,2.984891 \mathrm{lH}, 0,2.861568,-0.259489$ ,2.76661\H,0,4.351001,0.542264,3.340324 $\mathrm{H}, 0,2.759441,1.179705,3.817786$ IC, $0,4.001815,2.973567,2.036521 \backslash \mathrm{H}, 0,3.916828,3.664013,1.178903 \mathrm{lH}, 0,3.5$ $67086,3.472076,2.922605 \backslash \mathrm{H}, 0,5.076773,2.80233,2.230821 \backslash \mathrm{C}, 0,4.401928,2.0$ $47031,-4.097492 \mathrm{H}, 0,4.600291,2.964729,-3.515343 \mathrm{H}, 0,5.368941,1.535934$, $-4.261762 \mathrm{H}, 0,4.005252,2.347442,-5.084867 \backslash \mathrm{C}, 0,3.078029,-0.12767,-4.207$ $067 \mathrm{H}, 0,2.698085,0.17316,-5.201039 \mathrm{H}, 0,3.970649,-0.758532,-4.372067 \backslash \mathrm{H}$, $0,2.296329,-0.72408,-3.708653 \backslash \mathrm{C}, 0,-2.71952,-3.299364,1.764291 \backslash \mathrm{H}, 0,-3.3$ $61215,-4.194437,1.668865 \backslash \mathrm{H}, 0,-1.949162,-3.32764,0.97716 \backslash \mathrm{H}, 0,-2.215962$, $-3.359542,2.745531 \backslash \mathrm{H}, 0,-2.835701,-1.161555,1.852915 \mathrm{IV}$ Version=ES64L-G09 RevD.01IState=1-AlHF=-3216.0970767\RMSD=2.351e-09lDipole=2.3432935, -1 . $9836445,4.6565248 \mid$ Quadrupole $=-8.428126,9.3776108,-0.9494848,-19.947016$ 2,-2.2136709,-12.9820098|PG=C01 [X(C65H72N1O4P1)]॥@

\section{Conf1}

1\1\GINC-WORKER2ISPIRTPSSTPSSIdef2SVPIC65H72N1O4P1\JHIOEI05-May-201910 ॥\#p tpsstpss/def2svp int=ultrafine empiricaldispersion=gd3 scrf=(smd, solvent=ch2cl2, read, externaliteration, dovacuum) $\backslash$ titlell0,1 $\mathrm{P}, 0,-0.199$ $09,-0.47727,-1.12018210,0,-1.493382,0.546728,-1.40909210,0,0.738125,0$. $544853,-0.130925 \backslash 0,0,0.430098,-0.740852,-2.454853 \backslash 0,0,-0.592234,-1.575$ $552,-0.136942 \backslash \mathrm{C}, 0,-1.424315,2.31929,0.191846 \backslash \mathrm{C}, 0,-2.075827,1.207383,-0$ $.346734|\mathrm{C}, 0,-3.356371,0.754095,0.099124| \mathrm{C}, 0,-3.956484,1.443786,1.14378$ $2 \mathrm{H}, 0,-4.950319,1.142061,1.489607 \backslash \mathrm{C}, 0,-3.302904,2.524301,1.803354 \backslash \mathrm{C}, 0$, $-2.009656,2.965057,1.340575 \backslash \mathrm{C}, 0,-1.340308,3.986777,2.080209 \backslash \mathrm{H}, 0,-0.339$ $754,4.3017,1.770546 \backslash \mathrm{C}, 0,-1.92831,4.564732,3.196311 \mathrm{IH}, 0,-1.389153,5.338$ $473,3.753867 \backslash \mathrm{C}, 0,-3.218763,4.155427,3.627449 \backslash \mathrm{H}, 0,-3.674264,4.622937,4$. $507195 \backslash \mathrm{C}, 0,-3.888327,3.152988,2.943267 \backslash \mathrm{H}, 0,-4.874369,2.81166,3.27843 \backslash \mathrm{C}$ $, 0,-0.125295,2.768972,-0.385262 \backslash C, 0,0.938511,1.865297,-0.476927 \backslash C, 0,2$. $270116,2.266493,-0.811345 \backslash \mathrm{C}, 0,2.482726,3.590885,-1.167809 \backslash \mathrm{H}, 0,3.495238$ ,3.926542,-1.415386IC,0,1.411497,4.528983,-1.234056IC,0,0.085318,4.120 $948,-0.838447 \backslash \mathrm{C}, 0,-0.975039,5.071284,-0.944364 \backslash \mathrm{H}, 0,-1.992182,4.765803$, $-0.681369 \mid \mathrm{C}, 0,-0.735513,6.363093,-1.389523 \backslash \mathrm{H}, 0,-1.566425,7.072636,-1.4$ $70559 \backslash \mathrm{C}, 0,0.576812,6.772069,-1.749081 \backslash \mathrm{H}, 0,0.753434,7.796949,-2.093458 \backslash$ C, $0,1.626189,5.869482,-1.674893 \mathrm{H}, 0,2.640195,6.168766,-1.963898 \mathrm{IC}, 0,3$. $352282,-4.410132,-1.484189 \mathrm{IC}, 0,2.920414,-3.072292,-1.386051 \mathrm{H}, 0,3.0053$ $83,-2.406863,-2.248915 \backslash \mathrm{C}, 0,2.392707,-2.56762,-0.195839 \mathrm{H}, 0,2.129952,-1$ $.509049,-0.131729 \backslash \mathrm{C}, 0,2.277042,-3.396114,0.942768 \mid \mathrm{C}, 0,2.747109,-4.7299$ $44,0.865655 \backslash \mathrm{H}, 0,2.676607,-5.388547,1.736857 \backslash \mathrm{C}, 0,3.259185,-5.226917,-0$. $334474 \backslash \mathrm{H}, 0,3.59397,-6.269079,-0.384656 \backslash \mathrm{C}, 0,1.698308,-2.891416,2.198207$ IC, $0,2.303971,-3.315133,3.501411 \backslash \mathrm{H}, 0,2.134998,-2.566538,4.290897 \backslash \mathrm{H}, 0,3$ $.383905,-3.480312,3.368251 \mathrm{H}, 0,1.854212,-4.269105,3.834232 \mathrm{~N}, 0,0.67929$ $8,-2.06285,2.121891 \mathrm{H}, 0,0.259703,-1.843133,1.146046 \mathrm{IC}, 0,-0.033516,-1.4$ $17187,3.178793 \backslash \mathrm{C}, 0,-0.413255,-0.078217,2.960163 \backslash \mathrm{H}, 0,-0.136634,0.412282$ ,2.02306।C $, 0,-1.135192,0.603624,3.946669) \mathrm{H}, 0,-1.416115,1.647876,3.7813$ $29 \mathrm{IC}, 0,-1.503339,-0.049355,5.133863 \mathrm{IC}, 0,-1.152084,-1.394118,5.32975 \mathrm{IH}$, $0,-1.458359,-1.917253,6.241608 \mathrm{IC}, 0,-0.419613,-2.086752,4.355759 \mathrm{lH}, 0,-0$ $.18758,-3.144523,4.497316 \backslash \mathrm{C}, 0,3.390655,1.278157,-0.693354 \backslash \mathrm{C}, 0,3.989424$ $, 0.714096,-1.847492 \backslash \mathrm{C}, 0,3.847034,0.907937,0.604996 \mid C, 0,5.042567,-0.208$ $894,-1.681119|\mathrm{C}, 0,4.899189,-0.014709,0.715904| \mathrm{C}, 0,5.510149,-0.589983,-$ $0.414792 \backslash \mathrm{H}, 0,5.507701,-0.65291,-2.569513 \backslash \mathrm{H}, 0,5.256617,-0.295433,1.7120$ $3|\mathrm{C}, 0,-4.027843,-0.404248,-0.574097 \backslash \mathrm{C}, 0,-4.188562,-1.641986,0.110594| \mathrm{C}$ 
$, 0,-4.522483,-0.25728,-1.89793 \backslash \mathrm{C}, 0,-4.882465,-2.68461,-0.529308 \backslash \mathrm{C}, 0,-5$ $.198635,-1.336804,-2.497532 \backslash \mathrm{C}, 0,-5.399886,-2.555336,-1.830436 \mathrm{H}, 0,-5.0$ $13604,-3.63406,0.00196 \backslash \mathrm{H}, 0,-5.586546,-1.223177,-3.517466 \backslash \mathrm{H}, 0,-2.07496$, $0.486186,5.899092 \mathrm{CC}, 0,3.951983,-4.940453,-2.762547 \backslash \mathrm{H}, 0,3.643269,-5.983$ $539,-2.950038 \mathrm{H}, 0,3.666052,-4.322007,-3.629547 \backslash \mathrm{H}, 0,5.05746,-4.93469,-2$ $.697134 \backslash \mathrm{C}, 0,-4.36083,1.042265,-2.68854 \backslash \mathrm{H}, 0,-3.794236,1.75292,-2.063997$ IC, $0,-3.615395,-1.87779,1.510554 \backslash \mathrm{C}, 0,-6.147638,-3.704197,-2.501537 \backslash \mathrm{H}, 0$ $,-6.432486,-3.358679,-3.51322 \mathrm{lC}, 0,-4.716905,-1.818021,2.589583 \mathrm{H}, 0,-4$. $281007,-1.951888,3.596737 \backslash \mathrm{H}, 0,-5.250276,-0.851892,2.575479 \backslash \mathrm{H}, 0,-5.4648$ $78,-2.618067,2.43362 \mathrm{IC}, 0,-7.444704,-4.052129,-1.74222 \backslash \mathrm{H}, 0,-7.223268,-4$ $413566,-0.720964 \mathrm{H}, 0,-8.10433,-3.170206,-1.65394 \mathrm{H}, 0,-8.004245,-4.848$ $343,-2.266918 \mathrm{IC}, 0,-5.250143,-4.947831,-2.666207 \backslash \mathrm{H}, 0,-5.788112,-5.74864$ $9,-3.206215 \backslash \mathrm{H}, 0,-4.333707,-4.706371,-3.233745 \mathrm{|H}, 0,-4.943415,-5.351482$ $-1.683686 \mid \mathrm{C}, 0,-3.547055,0.821679,-3.980108 \mathrm{H}, 0,-4.078354,0.154964,-4.6$ $84317 \mathrm{VH}, 0,-3.375644,1.784719,-4.495736 \mathrm{H}, 0,-2.565158,0.374875,-3.74958$ $3 \backslash \mathrm{C}, 0,-5.727319,1.694553,-2.983577 \backslash \mathrm{H}, 0,-5.591686,2.658743,-3.507382 \backslash \mathrm{H}$ $0,-6.354258,1.048228,-3.625194 \backslash \mathrm{H}, 0,-6.284979,1.88853,-2.04953 \backslash \mathrm{C}, 0,6.65$ $1025,-1.593172,-0.266641 \mathrm{H}, 0,6.88578,-1.96838,-1.280715 \backslash \mathrm{C}, 0,3.543654,1$ $.104634,-3.258104 \mid \mathrm{H}, 0,2.606847,1.680023,-3.160936 \mathrm{IC}, 0,3.255191,1.55709$ $6,1.85877 \backslash \mathrm{H}, 0,2.20582,1.815804,1.636454 \backslash \mathrm{C}, 0,7.922009,-0.91716,0.289368$ $\mathrm{IH}, 0,8.757536,-1.63927,0.341885 \mathrm{IH}, 0,8.238142,-0.072188,-0.348152 \mathrm{H}, 0,7$ $.748858,-0.526487,1.309317 \backslash \mathrm{C}, 0,6.245798,-2.804101,0.598061 \mathrm{H}, 0,6.03043$ $5,-2.502271,1.639714 \backslash \mathrm{H}, 0,5.344499,-3.296725,0.195563 \mathrm{H}, 0,7.061178,-3.5$ $50009,0.627912 \backslash \mathrm{C}, 0,3.239935,0.634618,3.089866 \mathrm{lH}, 0,2.765935,-0.333718,2$ $.85926 \mathrm{VH}, 0,4.257406,0.434845,3.47231 \mathrm{H}, 0,2.668291,1.106443,3.908528 \mathrm{C}$ $0,3.995043,2.876558,2.17187 \mathrm{H}, 0,3.951532,3.572624,1.315793 \mathrm{H}, 0,3.54405$ $1,3.380381,3.046742 \mathrm{H}, 0,5.059557,2.681045,2.397962 \mathrm{IC}, 0,4.592194,2.0244$ $09,-3.919266 \backslash \mathrm{H}, 0,4.784747,2.92179,-3.304589 \backslash \mathrm{H}, 0,5.554067,1.49548,-4.05$ $4675 \mathrm{IH}, 0,4.24521,2.35878,-4.914401 \backslash \mathrm{C}, 0,3.22864,-0.115298,-4.145017 \backslash \mathrm{H}, 0$ $, 2.902987,0.221482,-5.146634 \mathrm{H}, 0,4.112511,-0.764049,-4.285965 \backslash \mathrm{H}, 0,2.40$ $9626,-0.704467,-3.70085 \backslash \mathrm{C}, 0,-2.837292,-3.205864,1.60331 \mathrm{H}, 0,-3.501951$, $-4.081497,1.486555 \backslash \mathrm{H}, 0,-2.053997,-3.247816,0.829682 \backslash \mathrm{H}, 0,-2.353935,-3.2$ $90421,2.592826 \mathrm{IH}, 0,-2.895582,-1.066727,1.712255$ IIVersion=ES64L-G09RevD $.01 \backslash$ State=1-AlHF=-3216.0970674\RMSD=2.411e-09|Dipole=2.1511303,-2.2072 $221,4.6778427 \backslash$ Quadrupole=-9.8259229,12.0811125,-2.2551896,-18.9268475, $-3.0530027,-12.2754142 \backslash \mathrm{PG}=\mathrm{C} 01$ [X(C65H72N1O4P1)]॥@

\section{Conf2}

1\1|GINC-WORKER0ISPIRTPSSTPSSIdef2SVPIC65H72N1O4P1\JHIOEI05-May-201910 ॥\#p tpsstpss/def2svp int=ultrafine empiricaldispersion=gd3 scrf=(smd, solvent=ch2cl2, read,externaliteration, dovacuum) |ltitlell0,1।P, $0,-0.155$ $228,-0.579395,-0.967524 \backslash 0,0,-1.438658,0.42075,-1.366627 \backslash 0,0,0.754101,0$ $.513805,-0.029774 \backslash 0,0,0.513917,-0.942732,-2.258201 \backslash 0,0,-0.587418,-1.60$ $3041,0.077683 \backslash \mathrm{C}, 0,-1.442528,2.273002,0.141688 \backslash \mathrm{C}, 0,-2.06485,1.127965,-0$ $.360351 \backslash \mathrm{C}, 0,-3.355556,0.682816,0.064064 \mathrm{IC}, 0,-3.997133,1.417735,1.05174$ $6 \mathrm{IH}, 0,-4.999718,1.124407,1.378874 \backslash \mathrm{C}, 0,-3.376638,2.537694,1.676662 \backslash \mathrm{C}, 0$, $-2.073467,2.970274,1.234824 \backslash \mathrm{C}, 0,-1.441817,4.038812,1.94066 \backslash \mathrm{H}, 0,-0.4350$ $51,4.34979,1.648056 \backslash \mathrm{C}, 0,-2.074505,4.668043,3.003484 \backslash \mathrm{H}, 0,-1.563772,5.47$ $7591,3.536325 \backslash \mathrm{C}, 0,-3.373777,4.26429,3.412975 \backslash \mathrm{H}, 0,-3.864271,4.77124,4.2$ $51036 \backslash \mathrm{C}, 0,-4.007391,3.21744,2.761794 \mathrm{IH}, 0,-4.999691,2.880162,3.082335 \mathrm{IC}$ $, 0,-0.134269,2.709553,-0.424584 \backslash \mathrm{C}, 0,0.941952,1.814709,-0.45113 \backslash \mathrm{C}, 0,2.2$ $70396,2.212857,-0.799977 \backslash \mathrm{C}, 0,2.471272,3.521275,-1.218696 \backslash \mathrm{H}, 0,3.482306$, $3.854152,-1.47594 \backslash \mathrm{C}, 0,1.393297,4.445004,-1.337501 \backslash \mathrm{C}, 0,0.066365,4.03988$ $8,-0.942596 \backslash \mathrm{C}, 0,-1.001988,4.972788,-1.109781 \backslash \mathrm{H}, 0,-2.020526,4.668775,-0$ $.851344 \backslash \mathrm{C}, 0,-0.768606,6.24621,-1.607759 \mathrm{H}, 0,-1.605769,6.941729,-1.7335$ $18 \mathrm{IC}, 0,0.544994,6.654106,-1.964138 \mathrm{IH}, 0,0.716416,7.664687,-2.350753 \backslash \mathrm{C}, 0$ $, 1.601508,5.766428,-1.835488 \mathrm{H}, 0,2.616792,6.062514,-2.12326 \backslash \mathrm{C}, 0,3.3800$ $33,-4.498677,-0.906033 \backslash \mathrm{C}, 0,2.9726,-3.150063,-0.884041 \mathrm{H}, 0,3.138571,-2$ $516376,-1.759657 \backslash \mathrm{C}, 0,2.373378,-2.594182,0.248702 \backslash \mathrm{H}, 0,2.128611,-1.52925$ $7,0.256458|\mathrm{C}, 0,2.163234,-3.379228,1.4049| \mathrm{C}, 0,2.604879,-4.725187,1.4047$ $02 \backslash \mathrm{H}, 0,2.458762,-5.351126,2.290671 \backslash \mathrm{C}, 0,3.18613,-5.273761,0.26038 \backslash \mathrm{H}, 0,3$ $.500474,-6.323492,0.268332 \backslash \mathrm{C}, 0,1.521772,-2.812329,2.602018 \mathrm{C}, 0,2.03259$ $-3.190567,3.958784 \mathrm{H}, 0,1.852763,-2.390769,4.69428 \mathrm{H}, 0,3.109442,-3.409$ $324,3.902064 \mathrm{IH}, 0,1.519917,-4.102952,4.316322 \mathrm{~N}, 0,0.534941,-1.96088,2.4$ $26616 \mathrm{IH}, 0,0.175942,-1.785633,1.416753 \mathrm{CC}, 0,-0.21323,-1.238951,3.405937 \mathrm{I}$ $\mathrm{C}, 0,-0.522414,0.101418,3.1021 \backslash \mathrm{H}, 0,-0.166834,0.535963,2.16361 \backslash \mathrm{C}, 0,-1.27$ $8039,0.855422,4.00759 \mathrm{H}, 0,-1.504575,1.900551,3.778827 \backslash \mathrm{C}, 0,-1.750818,0$ $273151,5.194446 \backslash \mathrm{C}, 0,-1.46658,-1.072443,5.474595 \mathrm{H}, 0,-1.853256,-1.53970$ $5,6.386283 \backslash \mathrm{C}, 0,-0.699195,-1.836435,4.584427 \backslash \mathrm{H}, 0,-0.51618,-2.893487,4.7$ $88585 \backslash \mathrm{C}, 0,3.417597,1.259815,-0.647689 \backslash \mathrm{C}, 0,4.039479,0.687945,-1.788694$ C, $0,3.906187,0.965319,0.655219 \mid \mathrm{C}, 0,5.165107,-0.13767,-1.605589 \mathrm{IC}, 0,5.0$ $34339,0.135074,0.784315 \backslash \mathrm{C}, 0,5.687539,-0.41705,-0.33139 \backslash \mathrm{H}, 0,5.648612,-0$ $.57182,-2.488369 \mathrm{|H}, 0,5.428801,-0.084659,1.783075 \mathrm{IC}, 0,-3.992199,-0.5131$ $01,-0.576324|C, 0,-4.176093,-1.71483,0.160919| C, 0,-4.428565,-0.437254,-$ $1.928834 \backslash \mathrm{C}, 0,-4.838244,-2.793398,-0.45754 \mid \mathrm{C}, 0,-5.069459,-1.548562,-2.5$ $03418 \mathrm{CC}, 0,-5.296624,-2.734295,-1.78317 \backslash \mathrm{H}, 0,-4.992347,-3.719527,0.10895$ $1 \mathrm{H}, 0,-5.404763,-1.482562,-3.545152 \backslash \mathrm{H}, 0,-2.350516,0.865497,5.893445 \backslash \mathrm{C}$ 
$0,4.044046,-5.094083,-2.122536 \backslash \mathrm{H}, 0,3.496121,-5.987742,-2.473542 \mathrm{H}, 0,4$. $095451,-4.368036,-2.94997 \backslash \mathrm{H}, 0,5.072992,-5.421324,-1.883397 \backslash \mathrm{C}, 0,-4.2410$ $03,0.821196,-2.778528 \backslash \mathrm{H}, 0,-3.707247,1.569361,-2.169105 \backslash \mathrm{C}, 0,-3.658689,-$ $1.880524,1.592103 \backslash \mathrm{C}, 0,-5.989846,-3.932305,-2.426483 \backslash \mathrm{H}, 0,-6.10599,-4.70$ $0324,-1.638714 \backslash \mathrm{C}, 0,-4.802213,-1.777047,2.62324 \mathrm{IH}, 0,-4.405745,-1.853795$ ,3.652485 $\mathrm{H}, 0,-5.342695,-0.818646,2.537555 \mathrm{IH}, 0,-5.536534,-2.591929,2.4$ $8001 \backslash \mathrm{C}, 0,-5.123525,-4.542873,-3.547949 \mathrm{H}, 0,-4.975629,-3.822056,-4.3732$ $7 \backslash \mathrm{H}, 0,-4.12733,-4.832203,-3.168357 \backslash \mathrm{H}, 0,-5.606618,-5.444278,-3.968369 \backslash \mathrm{C}$ $0,-7.397789,-3.576437,-2.944915 \backslash \mathrm{H}, 0,-7.902256,-4.474182,-3.346977 \backslash \mathrm{H}, 0$ $,-8.028368,-3.161826,-2.138014 \mathrm{IH}, 0,-7.350087,-2.827253,-3.756669 \mathrm{IC}, 0,-$ $3.371413,0.540385,-4.021301 \backslash \mathrm{H}, 0,-3.866838,-0.16954,-4.709253 \backslash \mathrm{H}, 0,-3.18$ $778,1.475924,-4.581414 \mathrm{IH}, 0,-2.396134,0.118589,-3.725892 \mathrm{CC}, 0,-5.598815$, $1.442687,-3.165234 \backslash \mathrm{H}, 0,-5.449154,2.382607,-3.727831 \backslash \mathrm{H}, 0,-6.189471,0.75$ $8859,-3.802499 \mathrm{IH}, 0,-6.200082,1.673453,-2.267273 \mathrm{IC}, 0,6.927798,-1.28999$, $-0.156235 \backslash \mathrm{H}, 0,7.16665,-1.298142,0.924082 \mathrm{lC}, 0,3.533673,0.964387,-3.2059$ 14\} \mathrm { H } , 0 , 2 . 5 6 7 1 7 9 , 1 . 4 8 9 1 5 7 , - 3 . 1 1 5 4 0 9 \backslash \mathrm { C } , 0 , 3 . 2 7 2 9 8 3 , 1 . 5 9 8 9 7 4 , 1 . 8 9 6 1 2 3 \backslash \mathrm { H } , 0 $2.223227,1.833055,1.651385 \backslash \mathrm{C}, 0,6.663771,-2.747211,-0.586875 \backslash \mathrm{H}, 0,7.5638$ 43,-3.369667,-0.429419\H,0,5.834836,-3.188119,-0.007521\H,0,6.396891,$2.803394,-1.657988 \mathrm{IC}, 0,8.144554,-0.70187,-0.899101 \backslash \mathrm{H}, 0,7.981635,-0.696$ $785,-1.992557 \backslash \mathrm{H}, 0,8.342965,0.337318,-0.580402 \backslash \mathrm{H}, 0,9.050899,-1.302042,-$ $0.698073 \mathrm{IC}, 0,3.251307,0.665426,3.119475 \mathrm{\backslash H}, 0,2.802298,-0.310245,2.86854$ $7 \backslash \mathrm{H}, 0,4.264805,0.481944,3.519907 \backslash \mathrm{H}, 0,2.653982,1.117515,3.931128 \mathrm{lC}, 0,3$. $972332,2.934604,2.230116 \mathrm{H}, 0,3.929303,3.631498,1.374335 \mathrm{H}, 0,3.488969,3$ $.424127,3.09581 \mathrm{H}, 0,5.03651,2.76724,2.479369 \mathrm{IC}, 0,4.503952,1.896014,-3$. $961884 \backslash \mathrm{H}, 0,4.661004,2.842682,-3.414765 \backslash \mathrm{H}, 0,5.491228,1.416118,-4.096913$ $\mathrm{IH}, 0,4.10649,2.141742,-4.963936 \backslash \mathrm{C}, 0,3.268215,-0.331829,-3.996825 \mathrm{IH}, 0,2$ $.892861,-0.08725,-5.007868 \mathrm{H}, 0,4.186636,-0.934521,-4.121598 \mathrm{H}, 0,2.5013$ $15,-0.93652,-3.485767 \backslash \mathrm{C}, 0,-2.879221,-3.198276,1.777228 \mathrm{H}, 0,-3.535891,-$ $4.08198,1.677976 \mathrm{lH}, 0,-2.066978,-3.273439,1.036588 \mathrm{H}, 0,-2.433127,-3.232$ $927,2.78703 \mathrm{H}, 0,-2.950683,-1.056251,1.781902$ IIVersion=ES64L-G09RevD.01 IState=1-AlHF=-3216.0959114\RMSD=2.239e-09|Dipole=1.8737331,-1.9436175 ,4.8971892lQuadrupole=-10.7882883,10.2639406,0.5243477,-17.931839,-2.4 719673,-14.8071693\PG=C01 [X(C65H72N1O4P1)]川@

Conf3

1\1\GINC-WORKER2ISPIRTPSSTPSSIdef2SVPIC65H72N1O4P1\JHIOEI05-May-2019\0

II\#p tpsstpss/def2svp int=ultrafine empiricaldispersion=gd3 scrf=(smd, solvent $=$ ch2cl2, read, externaliteration, dovacuum) \titlel| $0,1 \backslash \mathrm{P}, 0,-0.216$ $017,-0.537977,-1.069941 \backslash 0,0,-1.545208,0.445569,-1.341215 \backslash O, 0,0.728224$, $0.534188,-0.142033 \backslash 0,0,0.375417,-0.823764,-2.417141 \backslash 0,0,-0.549913,-1.6$ $1786,-0.045375 \backslash C, 0,-1.460074,2.267169,0.202987 \backslash C, 0,-2.103595,1.124232$, $-0.277059 \mid \mathrm{C}, 0,-3.354082,0.65363,0.231815 \backslash \mathrm{C}, 0,-3.929704,1.358872,1.2796$ $81 \mathrm{lH}, 0,-4.9008,1.043347,1.674288 \backslash \mathrm{C}, 0,-3.278431,2.473956,1.881475 \backslash \mathrm{C}, 0$, $2.016074,2.932585,1.355116 \backslash \mathrm{C}, 0,-1.34481,3.991941,2.038216 \backslash \mathrm{H}, 0,-0.36520$ $3,4.321958,1.680605 \backslash \mathrm{C}, 0,-1.90373,4.587637,3.160051 \mathrm{H}, 0,-1.363613,5.390$ $35,3.673981 \backslash \mathrm{C}, 0,-3.16536,4.159423,3.65365 \backslash \mathrm{H}, 0,-3.598375,4.64079,4.5372$ $7 \backslash \mathrm{C}, 0,-3.834796,3.120931,3.025518 \mathrm{IH}, 0,-4.797485,2.76471,3.409404 \backslash \mathrm{C}, 0,-$ $0.193975,2.728904,-0.434712 \mathrm{IC}, 0,0.887493,1.847288,-0.534545 \mathrm{IC}, 0,2.1979$ $39,2.268219,-0.924196 \backslash \mathrm{C}, 0,2.365036,3.584396,-1.332181 \backslash \mathrm{H}, 0,3.360407,3.9$ $35279,-1.624297 \backslash \mathrm{C}, 0,1.269747,4.494791,-1.391837 \backslash \mathrm{C}, 0,-0.031838,4.070158$ ,-0.936268\C,0,-1.116623,4.993769,-1.031009\H,0,-2.116253,4.675525,-0. $72036 \mathrm{IC}, 0,-0.922821,6.275998,-1.523608 \mathrm{VH}, 0,-1.771979,6.964709,-1.59448$ $3 \mid \mathrm{C}, 0,0.365582,6.701533,-1.945089 \backslash \mathrm{H}, 0,0.505813,7.71848,-2.327553 \backslash \mathrm{C}, 0,1$ $.437265,5.824677,-1.882317 \mathrm{H}, 0,2.433035,6.136714,-2.217518 \mathrm{IC}, 0,3.4111$, $-4.384289,-1.449758 \mathrm{IC}, 0,2.952858,-3.054539,-1.363125 \mathrm{IH}, 0,3.001324,-2.4$ $02597,-2.238851 \mathrm{lC}, 0,2.446443,-2.539442,-0.168112 \mathrm{~W}, 0,2.163647,-1.48535$ $,-0.116669 \mid \mathrm{C}, 0,2.379366,-3.349478,0.987916 \backslash \mathrm{C}, 0,2.87546,-4.674507,0.920$ $751 \backslash \mathrm{H}, 0,2.842768,-5.318682,1.804913 \mathrm{IC}, 0,3.365951,-5.182145,-0.283841 \backslash \mathrm{H}$ $, 0,3.721797,-6.217726,-0.324495 \backslash \mathrm{C}, 0,1.826044,-2.833339,2.250151 \backslash \mathrm{C}, 0,2$. 473182,-3.222961,3.543793\H,0,2.3237,-2.455353,4.319067\Н,0,3.549598,$3.385603,3.382833 \backslash \mathrm{H}, 0,2.039158,-4.170138,3.914636 \backslash \mathrm{N}, 0,0.792697,-2.0213$ $18,2.18778 \mathrm{IH}, 0,0.341234,-1.830817,1.220107 \backslash \mathrm{C}, 0,0.104729,-1.358414,3.25$ $0211 \backslash \mathrm{C}, 0,-0.301171,-0.03107,3.007645 \backslash \mathrm{H}, 0,-0.063842,0.436365,2.048141 \backslash \mathrm{C}$ $, 0,-0.997833,0.669144,3.999363 \backslash \mathrm{H}, 0,-1.298165,1.704723,3.815293 \backslash \mathrm{C}, 0,-1$. $316059,0.045572,5.216426 \backslash \mathrm{C}, 0,-0.940726,-1.288779,5.436788 \backslash \mathrm{H}, 0,-1.20859$ $4,-1.790187,6.372625 \backslash \mathrm{C}, 0,-0.232465,-1.999374,4.457734 \mathrm{H}, 0,0.017356,-3$. $049749,4.621315|C, 0,3.348211,1.31432,-0.805673| C, 0,3.940484,0.737388$,$1.956992 \backslash \mathrm{C}, 0,3.842952,0.992677,0.492005 \backslash \mathrm{C}, 0,5.022453,-0.15189,-1.78888$ $2 \backslash \mathrm{C}, 0,4.922287,0.102411,0.604406 \backslash \mathrm{C}, 0,5.525415,-0.486755,-0.523306 \mathrm{H}, 0$, $5.481701,-0.606574,-2.674989 \backslash \mathrm{H}, 0,5.308585,-0.141089,1.599504 \backslash \mathrm{C}, 0,-4.02$ $3718,-0.538553,-0.381813 \backslash \mathrm{C}, 0,-4.119539,-1.761472,0.336759 \backslash \mathrm{C}, 0,-4.5826$, $-0.436166,-1.686292 \backslash C, 0,-4.813502,-2.837467,-0.250334 \backslash C, 0,-5.256883,-1$ $.543266,-2.229887 \backslash \mathrm{C}, 0,-5.393267,-2.752741,-1.52611 \mathrm{M}, 0,-4.898937,-3.78$ $1143,0.301448 \backslash \mathrm{H}, 0,-5.690782,-1.4551,-3.232914 \backslash \mathrm{H}, 0,-1.868086,0.595453,5$ $.985848 \mathrm{IC}, 0,3.990507,-4.923636,-2.733656 \mathrm{H}, 0,3.689029,-5.972023,-2.902$ $768 \backslash \mathrm{H}, 0,3.680898,-4.31865,-3.601964 \mathrm{H}, 0,5.096927,-4.906188,-2.689827 \backslash \mathrm{C}$ $, 0,-4.4845,0.846376,-2.514792 \backslash \mathrm{H}, 0,-3.9087,1.585061,-1.932559 \backslash \mathrm{C}, 0,-3.48$ 
$2885,-1.948507,1.716044 \backslash \mathrm{C}, 0,-6.138215,-3.940819,-2.129115 \mathrm{H}, 0,-6.07947$ $4,-4.766401,-1.394843 \backslash \mathrm{C}, 0,-4.537279,-1.875794,2.840351 \mathrm{~W}, 0,-4.054849,-$ $1.975356,3.830106 \mathrm{H}, 0,-5.086529,-0.918619,2.823913 \mathrm{H}, 0,-5.278381,-2.69$ $0982,2.739727 \backslash \mathrm{C}, 0,-5.475179,-4.428541,-3.43379 \backslash \mathrm{H}, 0,-5.516569,-3.651146$ ,-4.218806 $\mathrm{H}, 0,-4.414142,-4.688336,-3.269654 \mathrm{IH}, 0,-5.993083,-5.325376,-$ $3.820802 \backslash \mathrm{C}, 0,-7.630588,-3.620008,-2.352945 \backslash \mathrm{H}, 0,-8.166855,-4.507882,-2$. 735567\} \backslash \mathrm { H } , 0 , - 8 . 1 1 7 2 0 2 , - 3 . 3 0 4 1 6 1 , - 1 . 4 1 2 6 8 5 \backslash \mathrm { H } , 0 , - 7 . 7 5 7 8 3 3 , - 2 . 8 0 6 0 1 6 , - 3 . 0 9 $0383 \backslash \mathrm{C}, 0,-3.719795,0.608832,-3.833364 \mathrm{|H}, 0,-4.265258,-0.087064,-4.49744$ $8 \mathrm{H}, 0,-3.590011,1.561089,-4.380223 \mathrm{lH}, 0,-2.719897,0.188066,-3.633076 \mathrm{IC}$, $0,-5.87656,1.46052,-2.770188 \mathrm{H}, 0,-5.784314,2.41461,-3.321105 \backslash \mathrm{H}, 0,-6.51$ $2317,0.784704,-3.371598 \backslash \mathrm{H}, 0,-6.402146,1.664206,-1.819788 \backslash \mathrm{C}, 0,6.691328$, $-1.45997,-0.371033 \backslash \mathrm{H}, 0,6.92411,-1.846488,-1.381283 \backslash \mathrm{C}, 0,3.452427,1.0718$ $79,-3.36809 \mathrm{H}, 0,2.514622,1.644512,-3.26601 \backslash \mathrm{C}, 0,3.261704,1.658368,1.742$ $106 \mathrm{IH}, 0,2.19927,1.876977,1.539959 \mathrm{IC}, 0,7.952002,-0.747467,0.161711 \mathrm{lH}, 0$, $8.803875,-1.449966,0.217675 \mathrm{H}, 0,8.243175,0.093926,-0.492268 \mathrm{H}, 0,7.7802$ $96,-0.344431,1.177055 \backslash \mathrm{C}, 0,6.320232,-2.664511,0.517729 \backslash \mathrm{H}, 0,6.110784,-2$. $349191,1.5566 \mathrm{IH}, 0,5.423923,-3.180719,0.134475 \mathrm{H}, 0,7.150208,-3.394065,0$ $.550331 \mathrm{lC}, 0,3.306762,0.769622,2.996773 \mathrm{H}, 0,2.871505,-0.223428,2.797831$ $\mathrm{IH}, 0,4.338125,0.623072,3.365899 \backslash \mathrm{H}, 0,2.72941,1.237826,3.813388 \backslash \mathrm{C}, 0,3.96$ $5295,3.008961,2.00063 \mathrm{H}, 0,3.877799,3.679165,1.127545 \mathrm{IH}, 0,3.519254,3.52$ $2205,2.872555 \backslash \mathrm{H}, 0,5.04088,2.854328,2.204956 \backslash \mathrm{C}, 0,4.472802,1.972527,-4.0$ $96002 \backslash \mathrm{H}, 0,4.676477,2.894661,-3.522756 \backslash \mathrm{H}, 0,5.434569,1.446298,-4.241906 \backslash$ $\mathrm{H}, 0,4.092494,2.266147,-5.091823 \mathrm{IC}, 0,3.120337,-0.185411,-4.195198 \mathrm{H}, 0,2$ $.766956,0.106904,-5.201402 \mathrm{H}, 0,4.003536,-0.836217,-4.33104 \mathrm{H}, 0,2.31662$ $3,-0.758791,-3.704731 \backslash \mathrm{C}, 0,-2.678331,-3.260246,1.8136 \mathrm{H}, 0,-3.331928,-4$. $150035,1.757559 \mathrm{H}, 0,-1.931124,-3.315641,1.005929 \backslash \mathrm{H}, 0,-2.147435,-3.3040$ 22,2.781115।H,0,-2.769819,-1.11994,1.863624IIVersion=ES64L-G09RevD.01। State=1-AlHF=-3216.0970157 $\backslash \mathrm{RMSD}=2.389 \mathrm{e}-09 \mathrm{VDipole}=2.3361036,-2.0319428$, 4.6541888|Quadrupole=-9.4883586,10.703515,-1.2151564,-19.9919649,-1.92 $95199,-12.598029 \mathrm{PG}=\mathrm{C} 01[\mathrm{X}(\mathrm{C} 65 \mathrm{H} 72 \mathrm{~N} 1 \mathrm{O} 4 \mathrm{P} 1)] 1 @$

\section{TRIP/1E Type I}

\section{Confo}

1\1|GINC-LOGINISPIRTPSSTPSSIdef2SVPIC65H72N1O4P1 IJHIOEI06-May-201910।I

\#p tpsstpss/def2svp int=ultrafine empiricaldispersion=gd3 scrf=(smd,so

Ivent=ch2cl2, read,externaliteration, dovacuum) \titlel|0,1\P,0,-0.10843

$9,-0.447366,-1.242731 \backslash 0,0,-1.431908,0.570095,-1.391101 \backslash 0,0,0.823429,0$. $472831,-0.160745 \backslash 0,0,0.504533,-0.533094,-2.608759 \backslash 0,0,-0.45731,-1.6674$ $14,-0.396129|C, 0,-1.312229,2.256433,0.304329| C, 0,-1.988344,1.187587,-0$ .291383 IC $, 0,-3.286663,0.753824,0.125556 \mid C, 0,-3.876454,1.410077,1.19781$ $\mathrm{IH}, 0,-4.878244,1.113224,1.525644 \backslash \mathrm{C}, 0,-3.198893,2.438397,1.913361 \backslash \mathrm{C}, 0,-$ $1.887489,2.858895,1.482717 \backslash \mathrm{C}, 0,-1.192634,3.818651,2.27983 \backslash \mathrm{H}, 0,-0.17906$ $7,4.115366,1.995523 \backslash \mathrm{C}, 0,-1.773362,4.360781,3.417199 \mathrm{|H}, 0,-1.214585,5.08$ $7395,4.017267 \backslash \mathrm{C}, 0,-3.082128,3.976408,3.814797 \backslash \mathrm{H}, 0,-3.531886,4.416673,4$ $.711386 \mathrm{IC}, 0,-3.77625,3.030518,3.07697 \backslash \mathrm{H}, 0,-4.77625,2.705804,3.386313 \mathrm{IC}$ $, 0,-0.009473,2.720243,-0.252886 \mid C, 0,1.041097,1.811262,-0.414385 \backslash C, 0,2$. $374166,2.213048,-0.740573 \mathrm{IC}, 0,2.602375,3.555336,-1.010021 \mathrm{H}, 0,3.617068$ $, 3.893658,-1.244154|C, 0,1.54403,4.509893,-1.004389| C, 0,0.215367,4.0953$ $77,-0.625037 \backslash \mathrm{C}, 0,-0.833204,5.063391,-0.669616 \mathrm{lH}, 0,-1.853229,4.756108,-$ $0.420771 \backslash \mathrm{C}, 0,-0.579725,6.376061,-1.040006 \backslash \mathrm{H}, 0,-1.402474,7.098707,-1.07$ $6085 \mathrm{IC}, 0,0.735988,6.78997,-1.380543 \mathrm{IH}, 0,0.923725,7.831021,-1.665393 \mathrm{IC}$, $0,1.774135,5.871738,-1.365401 \mathrm{H}, 0,2.790502,6.174335,-1.642256 \mathrm{IC}, 0,-1.4$ $84247,-0.672644,4.67252 \backslash \mathrm{C}, 0,-0.968356,-1.891613,5.160822 \backslash \mathrm{H}, 0,-1.320245$ $,-2.279614,6.123 \backslash \mathrm{C}, 0,-0.012532,-2.614593,4.439549 \backslash \mathrm{H}, 0,0.362478,-3.5587$ $86,4.845938 \backslash \mathrm{C}, 0,0.428027,-2.154778,3.17601 \backslash \mathrm{C}, 0,-0.078816,-0.926491,2.6$ $8633 \backslash \mathrm{H}, 0,0.274329,-0.519187,1.736303 \backslash \mathrm{C}, 0,-1.006796,-0.200796,3.429945 \backslash$ $\mathrm{H}, 0,-1.371773,0.752816,3.037219 \mathrm{IC}, 0,1.403641,-2.937189,2.402355 \backslash \mathrm{C}, 0,2$. $426712,-3.767474,3.117788 \mathrm{H}, 0,3.325139,-3.915549,2.498661 \mathrm{H}, 0,2.01044$ $-4.762785,3.3587 \backslash \mathrm{H}, 0,2.704368,-3.280514,4.064871 \mathrm{~N}, 0,1.372655,-2.85735$ $2,1.090101 \mathrm{WH}, 0,0.606212,-2.27416,0.599507 \backslash \mathrm{C}, 0,2.20945,-3.520338,0.1374$ $94 \mathrm{IC}, 0,2.717731,-2.750206,-0.925311 \backslash \mathrm{H}, 0,2.5404,-1.672167,-0.960912 \backslash \mathrm{C}, 0$ $, 3.477148,-3.373817,-1.922648 \mathrm{H}, 0,3.87709,-2.770691,-2.742866 \mid \mathrm{C}, 0,3.73$ $0136,-4.753178,-1.864869 \backslash \mathrm{C}, 0,3.213604,-5.515541,-0.805085 \mathrm{lH}, 0,3.39546$, $-6.594493,-0.761647 \backslash \mathrm{C}, 0,2.444424,-4.907006,0.195838 \backslash \mathrm{H}, 0,2.004112,-5.50$ $8078,0.99558|C, 0,3.48478,1.206269,-0.711889| C, 0,4.057083,0.722082,-1.9$ $16024|C, 0,3.96308,0.739105,0.545938 I C, 0,5.115808,-0.204856,-1.837164| C$ $, 0,5.014713,-0.191689,0.569146 \mathrm{IC}, 0,5.604654,-0.681054,-0.610959 \mathrm{H}, 0,5$. $5625,-0.584316,-2.763753 \backslash \mathrm{H}, 0,5.383773,-0.549263,1.535242 \backslash \mathrm{C}, 0,-3.980424$ $,-0.362854,-0.592434 \backslash \mathrm{C}, 0,-4.216442,-1.600085,0.066686 \mid C, 0,-4.420837,-0$ $.179444,-1.933762 \backslash \mathrm{C}, 0,-4.908221,-2.615482,-0.622588 \backslash \mathrm{C}, 0,-5.101691,-1.2$ $26538,-2.577836 \mathrm{IC}, 0,-5.361243,-2.453411,-1.94095 \mathrm{IH}, 0,-5.093554,-3.5707$ $58,-0.11618 \mathrm{H}, 0,-5.446515,-1.075668,-3.607584 \mathrm{H}, 0,4.325067,-5.234896,--$ 
$2.647885 \backslash \mathrm{C}, 0,-2.523635,0.111107,5.433506 \backslash \mathrm{H}, 0,-3.465263,0.161198,4.8551$ $97 \backslash \mathrm{H}, 0,-2.742457,-0.343867,6.413387 \backslash \mathrm{H}, 0,-2.192947,1.153222,5.592985 \mathrm{IC}$, $0,-4.211482,1.135438,-2.687878 \mathrm{H}, 0,-3.636375,1.814774,-2.037095 \backslash \mathrm{C}, 0,-3$ $.720329,-1.878277,1.487151 \backslash \mathrm{C}, 0,-6.116791,-3.571614,-2.654339 \mathrm{H}, 0,-6.14$ $501,-4.434986,-1.963017 \backslash \mathrm{C}, 0,-4.88892,-1.972418,2.489305 \backslash \mathrm{H}, 0,-4.50968,-$ $2.149673,3.512606 \mathrm{lH}, 0,-5.485721,-1.042881,2.50159 \mathrm{H}, 0,-5.568433,-2.806$ $711,2.233482 \mathrm{IC}, 0,-5.395604,-4.024369,-3.940333 \backslash \mathrm{H}, 0,-5.354973,-3.208632$ $,-4.685447 \backslash \mathrm{H}, 0,-4.358973,-4.340993,-3.727378 \mathrm{H}, 0,-5.926608,-4.875625,-$ 4.404737\C, $0,-7.574175,-3.161769,-2.952167 \backslash \mathrm{H}, 0,-8.129002,-3.99836,-3.4$ $15566 \backslash \mathrm{H}, 0,-8.103784,-2.868803,-2.027948 \backslash \mathrm{H}, 0,-7.611361,-2.305058,-3.650$ $321 \backslash \mathrm{C}, 0,-3.389343,0.933473,-3.976782 \backslash \mathrm{H}, 0,-3.927569,0.299655,-4.705724$ $\mathrm{H}, 0,-3.192509,1.907261,-4.462227 \mathrm{H}, 0,-2.41928,0.459801,-3.750816 \mathrm{IC}, 0,-$ $5.559098,1.82818,-2.97985 \mathrm{H}, 0,-5.395071,2.802725,-3.475716 \mathrm{H}, 0,-6.1932$ $68,1.213746,-3.645309 \mathrm{H}, 0,-6.122913,2.009802,-2.046998 \mathrm{lC}, 0,6.749983,-1$ $.689999,-0.5687 \backslash \mathrm{H}, 0,6.839995,-2.115217,-1.586294 \backslash \mathrm{C}, 0,3.558595,1.188375$ ,-3.285056।H $0,2.586931,1.687553,-3.127134 \backslash \mathrm{C}, 0,3.381855,1.277237,1.856$ $181 \backslash \mathrm{H}, 0,2.315401,1.499256,1.678472 \mathrm{lC}, 0,8.085058,-0.989475,-0.236153 \mathrm{lH}$ $0,8.921627,-1.712275,-0.250661 \backslash \mathrm{H}, 0,8.308839,-0.189779,-0.964591 \backslash \mathrm{H}, 0,8$. $047304,-0.531347,0.769749 \mathrm{IC}, 0,6.482404,-2.855178,0.404198 \backslash \mathrm{H}, 0,6.455676$ ,-2.509525,1.453758\H,0,5.520417,-3.349461,0.182615।H,0,7.283275,-3.61 $2873,0.327505 \mathrm{IC}, 0,3.443148,0.272343,3.019914 \mathrm{IH}, 0,3.039284,-0.710206,2$ $723786 \mathrm{IH}, 0,4.476195,0.121119,3.382673 \mathrm{IH}, 0,2.84605,0.644106,3.871358 \mathrm{IC}$, $0,4.068608,2.604094,2.247969 \mathrm{H}, 0,3.976026,3.357404,1.446343 \mathrm{H}, 0,3.6144$ $45,3.02218,3.165276 \mathrm{H}, 0,5.145191,2.441447,2.440588 \mathrm{IC}, 0,4.530792,2.2195$ $51,-3.896614 \mathrm{H}, 0,4.668989,3.089544,-3.230829 \backslash \mathrm{H}, 0,5.524911,1.767212,-4$. $071603 \backslash \mathrm{H}, 0,4.149514,2.588176,-4.866831 \backslash \mathrm{C}, 0,3.306982,0.018743,-4.255803$ $\mathrm{IH}, 0,2.887194,0.40279,-5.203896 \backslash \mathrm{H}, 0,4.238658,-0.521907,-4.504231 \backslash \mathrm{H}, 0,2$ $.578078,-0.686219,-3.824415 \backslash \mathrm{C}, 0,-2.83138,-3.136987,1.547764 \mathrm{H}, 0,-3.405$ $939,-4.055154,1.324938 \backslash \mathrm{H}, 0,-2.002695,-3.053794,0.825605 \mathrm{lH}, 0,-2.40214,-$ $3.249396,2.560089 \mathrm{IH}, 0,-3.08899,-1.028144,1.791982$ IIVersion=ES64L-G09Re vD.01\State=1-AlHF=-3216.0973304IRMSD=6.007e-09|Dipole=1.708134,-2.421 $8302,5.0102926 \mid$ Quadrupole=-8.2872772,12.7295441,-4.442267,-17.3134219, $-4.928402,-13.6000131 \mid \mathrm{PG}=\mathrm{C} 01$ [X(C65H72N1O4P1)]川@

Conf1

1\1\GINC-WORKER2ISPIRTPSSTPSSIdef2SVPIC65H72N1O4P1\JHIOEI05-May-2019\0

II\#p tpsstpss/def2svp int=ultrafine empiricaldispersion=gd3 scrf=(smd, solvent $=\mathrm{ch} 2 \mathrm{cl} 2$, read,externaliteration, dovacuum) ) Ititlell0,1|P, $0,-0.101$ $954,-0.381699,-1.301615 \backslash 0,0,-1.385719,0.68041,-1.476842 \backslash 0,0,0.823327,0$ $.492836,-0.1765910,0,0.554728,-0.465515,-2.64740310,0,-0.517626,-1.600$ $693,-0.484293 \backslash \mathrm{C}, 0,-1.263055,2.340934,0.242693 \backslash \mathrm{C}, 0,-1.95202,1.296689,-0$ $.381248 \mathrm{IC}, 0,-3.266946,0.885425,0.004014 \mathrm{IC}, 0,-3.867256,1.546746,1.06707$ $5 \mathrm{IH}, 0,-4.883068,1.270843,1.3685 \backslash \mathrm{C}, 0,-3.184311,2.554967,1.805894 \backslash \mathrm{C}, 0,-1$ $.852605,2.946971,1.412144 \backslash \mathrm{C}, 0,-1.156077,3.883852,2.234627 \backslash \mathrm{H}, 0,-0.1288$, $4.158197,1.979161 \backslash \mathrm{C}, 0,-1.753245,4.432632,3.360238 \backslash \mathrm{H}, 0,-1.192982,5.1414$ $83,3.979868 \backslash \mathrm{C}, 0,-3.08106,4.078039,3.720394 \backslash \mathrm{H}, 0,-3.543724,4.5236,4.6077$ $56 \backslash \mathrm{C}, 0,-3.777705,3.153619,2.958148 \mathrm{IH}, 0,-4.793118,2.851335,3.238783 \backslash \mathrm{C}, 0$ $0.070069,2.767785,-0.272017 \backslash \mathrm{C}, 0,1.094351,1.825007,-0.40823 \backslash \mathrm{C}, 0,2.4504$ $14,2.182934,-0.686861 \backslash \mathrm{C}, 0,2.732089,3.518056,-0.940661 \mathrm{H}, 0,3.764549,3.8$ $23737,-1.139445 \backslash \mathrm{C}, 0,1.706396,4.5076,-0.962639 \backslash \mathrm{C}, 0,0.353436,4.136701,-0$ $.626142 \backslash \mathrm{C}, 0,-0.659297,5.14074,-0.693906 \backslash \mathrm{H}, 0,-1.695854,4.867359,-0.4757$ $06 \backslash \mathrm{C}, 0,-0.350395,6.445938,-1.048306 \backslash \mathrm{H}, 0,-1.14661,7.196585,-1.102914 \mathrm{IC}$, $0,0.987973,6.816013,-1.348551 \backslash \mathrm{H}, 0,1.219782,7.851278,-1.621702 \mathrm{CC}, 0,1.99$ $321,5.862532,-1.308233 \backslash \mathrm{H}, 0,3.027119,6.13154,-1.553061 \backslash \mathrm{C}, 0,-1.57454,-0$. $652599,4.584802 \backslash \mathrm{C}, 0,-1.107824,-1.897052,5.058124 \backslash \mathrm{H}, 0,-1.49024,-2.29172$ $2,6.005855 \mathrm{IC}, 0,-0.163586,-2.637863,4.339718 \mathrm{H}, 0,0.172505,-3.601691,4.7$ $33764 \backslash \mathrm{C}, 0,0.31245,-2.170405,3.09205 \backslash \mathrm{C}, 0,-0.143056,-0.916083,2.618567 \backslash \mathrm{H}$ $, 0,0.23953,-0.50428,1.681967 \backslash \mathrm{C}, 0,-1.058425,-0.172837,3.360745 \mathrm{\backslash H}, 0,-1.3$ $84547,0.799991,2.98052 \backslash \mathrm{C}, 0,1.268749,-2.975212,2.317035 \backslash \mathrm{C}, 0,2.254938,-3$ $850896,3.030236 \mathrm{H}, 0,3.158867,-4.010372,2.421629 \backslash \mathrm{H}, 0,1.80621,-4.839041$ ,3.240344\H,0,2.53092,-3.395106,3.993061\N,0,1.251656,-2.879156,1.0055 $95 \mathrm{IH}, 0,0.510859,-2.262893,0.515859 \mathrm{lC}, 0,2.072056,-3.56132,0.052336 \backslash \mathrm{C}, 0$, $2.612456,-2.799783,-1.000854 \backslash \mathrm{H}, 0,2.471471,-1.716263,-1.028694 \backslash \mathrm{C}, 0,3.35$ $6902,-3.439834,-1.9989 \mathrm{H}, 0,3.783034,-2.843566,-2.810815 \backslash \mathrm{C}, 0,3.56236,-4$ $.827556,-1.95245 \backslash \mathrm{C}, 0,3.014219,-5.581007,-0.902325 \backslash \mathrm{H}, 0,3.159199,-6.6658$ $13,-0.867211 \backslash \mathrm{C}, 0,2.260829,-4.95535,0.10005 \backslash \mathrm{H}, 0,1.79691,-5.548425,0.892$ $258 \mid C, 0,3.524456,1.138065,-0.62299 \backslash C, 0,4.112799,0.627485,-1.808033 \backslash C, 0$ $3.952326,0.663004,0.650547 \backslash \mathrm{C}, 0,5.138819,-0.333097,-1.695663 \backslash \mathrm{C}, 0,4.972$ $217,-0.300692,0.706965 \backslash \mathrm{C}, 0,5.579985,-0.814652,-0.453896 \backslash \mathrm{H}, 0,5.599313,-$ $0.73152,-2.607321 \mathrm{lH}, 0,5.30627,-0.663537,1.684094 \mathrm{lC}, 0,-3.966786,-0.2152$ $41,-0.733828 \mid C, 0,-4.210479,-1.461862,-0.091261 \backslash C, 0,-4.404503,-0.010114$ $,-2.070005 \backslash C, 0,-4.916526,-2.458668,-0.787945 \backslash C, 0,-5.100324,-1.043492,-$ $2.725746 \backslash \mathrm{C}, 0,-5.37396,-2.272556,-2.10443 \backslash \mathrm{H}, 0,-5.105201,-3.416059,-0.28$ $8621 \mathrm{H}, 0,-5.445259,-0.884575,-3.754983 \mathrm{H}, 0,4.145345,-5.322255,-2.73634$ IC $, 0,-2.5983,0.152558,5.344667 \backslash \mathrm{H}, 0,-3.501252,0.309655,4.726329 \backslash \mathrm{H}, 0,-2$ $898147,-0.346618,6.280574 \backslash \mathrm{H}, 0,-2.20446,1.155391,5.591544 \backslash \mathrm{C}, 0,-4.169002$ ,1.308093,-2.809557\H,0,-3.594201,1.973064,-2.143499\C,0,-3.707342,-1. 
$758119,1.322827 \backslash \mathrm{C}, 0,-6.133401,-3.373288,-2.840045 \backslash \mathrm{H}, 0,-6.381927,-2.981$ $24,-3.8444 \backslash \mathrm{C}, 0,-4.859849,-1.762402,2.347931 \backslash \mathrm{H}, 0,-4.472203,-1.947593,3$. $366632 \backslash \mathrm{H}, 0,-5.398337,-0.798457,2.356639 \backslash \mathrm{H}, 0,-5.593484,-2.556844,2.1154$ $88 \mathrm{IC}, 0,-7.459068,-3.71985,-2.131308 \mathrm{H}, 0,-7.276344,-4.135655,-1.12312 \backslash \mathrm{H}$ $, 0,-8.097893,-2.82561,-2.018345 \backslash \mathrm{H}, 0,-8.024631,-4.474811,-2.707971 \backslash \mathrm{C}, 0$, $-5.261677,-4.631745,-3.030385 \backslash \mathrm{H}, 0,-5.803828,-5.397618,-3.615088 \mathrm{H}, 0,-4$ $.32467,-4.391611,-3.563809 \backslash \mathrm{H}, 0,-4.991463,-5.079924,-2.05642 \backslash \mathrm{C}, 0,-3.329$ $358,1.104892,-4.087181 \backslash \mathrm{H}, 0,-3.865235,0.485093,-4.829706 \backslash \mathrm{H}, 0,-3.110288$, $2.079674,-4.561024 \mathrm{IH}, 0,-2.370144,0.614757,-3.849938 \backslash \mathrm{C}, 0,-5.50193,2.022$ $158,-3.115874 \mathrm{|H}, 0,-5.316897,2.998744,-3.600143 \backslash \mathrm{H}, 0,-6.134353,1.423003$, $-3.796673 \backslash \mathrm{H}, 0,-6.077263,2.203131,-2.189931 \backslash \mathrm{C}, 0,6.686791,-1.862333,-0.3$ $65121 \backslash \mathrm{H}, 0,6.88289,-2.212807,-1.396109 \backslash \mathrm{C}, 0,3.668749,1.106805,-3.191069 \backslash$ $\mathrm{H}, 0,2.702524,1.624359,-3.061616 \mathrm{IC}, 0,3.352047,1.226652,1.941528 \mathrm{H}, 0,2.2$ $96412,1.474268,1.734008 \mathrm{IC}, 0,7.993801,-1.246114,0.177301 \mathrm{lH}, 0,8.805109$, $1.997112,0.188151 \backslash \mathrm{H}, 0,8.32253,-0.394141,-0.444301 \backslash \mathrm{H}, 0,7.857508,-0.8787$ $8,1.211528 \mid \mathrm{C}, 0,6.264536,-3.086687,0.472739 \mathrm{IH}, 0,6.088739,-2.812435,1.52$ $9212 \mathrm{H}, 0,5.337707,-3.538293,0.078956 \mathrm{H}, 0,7.056192,-3.857928,0.456882 \mathrm{CC}$ $, 0,3.358395,0.231043,3.114917 \backslash \mathrm{H}, 0,2.938889,-0.744647,2.818041 \backslash \mathrm{H}, 0,4.37$ $7691,0.059514,3.506446 \mathrm{lH}, 0,2.748106,0.624954,3.94682 \mathrm{lC}, 0,4.063015,2.53$ $8174,2.342035 \mathrm{H}, 0,4.008571,3.289191,1.535021 \mathrm{H}, 0,3.598574,2.972964,3.2$ $46334 \mathrm{IH}, 0,5.130209,2.348881,2.560646 \mathrm{IC}, 0,4.681318,2.119497,-3.766704 \mathrm{IH}$ $, 0,4.820002,2.981882,-3.091351 \mathrm{H}, 0,5.669515,1.645399,-3.91578 \backslash \mathrm{H}, 0,4.33$ $6425,2.502307,-4.74499 \mid \mathrm{C}, 0,3.424265,-0.052506,-4.175599 \mathrm{IH}, 0,3.04511,0$. $345224,-5.135089 \mathrm{H}, 0,4.351318,-0.61281,-4.396028 \mathrm{H}, 0,2.665418,-0.74233$ $3,-3.772737 \backslash \mathrm{C}, 0,-2.906032,-3.073623,1.389567 \backslash \mathrm{H}, 0,-3.546387,-3.955091,1$ $.200856 \mathrm{H}, 0,-2.090188,-3.062133,0.64872 \mathrm{H}, 0,-2.462694,-3.194868,2.3946$ $7 \backslash \mathrm{H}, 0,-3.014469,-0.948273,1.602377 \| I V e r s i o n=E S 64 L-G 09 R e v D .011 S t a t e=1-A$ $\backslash \mathrm{HF}=-3216.0974305 \backslash \mathrm{RMSD}=6.114 \mathrm{e}-09 \mathrm{|Dipole}=1.5336404,-2.5800941,5.0002904$ IQuadrupole=-9.3084686,14.6792822,-5.3708136,-16.250315,-5.5550033,-12 .5917013\PG=C01 [X(C65H72N1O4P1)]॥@

\section{Conf2}

1\1\GINC-WORKER0ISPIRTPSSTPSSIdef2SVPIC65H72N1O4P1\JHIOEI06-May-201910 II tpsstpss/def2svp int=ultrafine empiricaldispersion=gd3 scrf=(smd, solvent $=$ ch2cl2, read,externaliteration, dovacuum) ||titlell0,1।P, $0,-0.065$ $194,-0.472512,-1.22955 \backslash 0,0,-1.286101,0.637643,-1.51914 \backslash 0,0,0.849498,0$. $412355,-0.101712 \backslash 0,0,0.64751,-0.656324,-2.535968 \backslash 0,0,-0.584102,-1.6221$ $75,-0.371724 \backslash \mathrm{C}, 0,-1.205624,2.333403,0.164606 \backslash \mathrm{C}, 0,-1.893667,1.296917,-0$ $.471989|\mathrm{C}, 0,-3.240456,0.936478,-0.151613| \mathrm{C}, 0,-3.878041,1.646853,0.8565$ $22 \backslash \mathrm{H}, 0,-4.918442,1.414009,1.105324 \backslash \mathrm{C}, 0,-3.205642,2.655461,1.604662 \backslash \mathrm{C}, 0$ $,-1.840855,2.992651,1.279752 \mathrm{IC}, 0,-1.161246,3.929772,2.115875 \mathrm{IH}, 0,-0.11$ $1806,4.161719,1.913904 \backslash \mathrm{C}, 0,-1.804039,4.531229,3.188242 \backslash \mathrm{H}, 0,-1.25691,5$. $239152,3.820539 \mid \mathrm{C}, 0,-3.162217,4.231455,3.478097 \backslash \mathrm{H}, 0,-3.66028,4.717706$ 4.323881।C $, 0,-3.844441,3.307768,2.701975 \mathrm{lH}, 0,-4.88396,3.046624,2.93058$ $5 \mid C, 0,0.164754,2.705571,-0.291004 \backslash C, 0,1.166606,1.729947,-0.357648 \backslash C, 0$ $2.541686,2.044705,-0.587207 \backslash \mathrm{C}, 0,2.871435,3.366844,-0.854868 \backslash \mathrm{H}, 0,3.9202$ $32,3.638935,-1.012725 \backslash \mathrm{C}, 0,1.8778,4.383402,-0.947264 \backslash \mathrm{C}, 0,0.50142,4.0566$ $38,-0.666922 \backslash \mathrm{C}, 0,-0.478423,5.085616,-0.809599 \mathrm{IH}, 0,-1.531745,4.846673,-$ $0.637623 \backslash \mathrm{C}, 0,-0.116648,6.372473,-1.180468 \backslash \mathrm{H}, 0,-0.888677,7.141732,-1.29$ 2788\C, $0,1.244363,6.699645,-1.42358 \backslash \mathrm{H}, 0,1.517731,7.720958,-1.7104 \backslash \mathrm{C}, 0$ $2.219124,5.720826,-1.311437 \backslash \mathrm{H}, 0,3.270787,5.954763,-1.512885 \backslash \mathrm{C}, 0,-1.921$ $889,-0.360087,4.605463 \mathrm{IC}, 0,-1.576178,-1.614399,5.151615 \mathrm{IH}, 0,-2.04697,-$ $1.945026,6.083993 \backslash \mathrm{C}, 0,-0.641997,-2.444282,4.523918 \backslash \mathrm{H}, 0,-0.400224,-3.41$ $3352,4.970665|C, 0,-0.050615,-2.056622,3.298533| C, 0,-0.383713,-0.793036$ ,2.752458\H,0,0.0875,-0.44484,1.829588\C,0,-1.29206,0.037678,3.405505। $\mathrm{H}, 0,-1.52323,1.015058,2.972407 \backslash \mathrm{C}, 0,0.901489,-2.948165,2.620381 \backslash \mathrm{C}, 0,1.7$ $60375,-3.874738,3.42588 \mathrm{H}, 0,2.67556,-4.150214,2.880136 \mathrm{H}, 0,1.204104,-4$ $.80331,3.651043 \mathrm{IH}, 0,2.024746,-3.401339,4.383722 \mathrm{WN}, 0,0.994682,-2.882512$ , 1.311177\\, $0,0.324887,-2.248724,0.745266 \backslash \mathrm{C}, 0,1.855139,-3.621699,0.439$ $035 \mathrm{IC}, 0,2.551866,-2.894836,-0.544812 \mathrm{H}, 0,2.49388,-1.803106,-0.56625 \backslash \mathrm{C}$, $0,3.336754,-3.582766,-1.477343 \backslash \mathrm{H}, 0,3.881387,-3.015547,-2.237515 \mathrm{IC}, 0,3$. $428466,-4.983176,-1.431963 \backslash \mathrm{C}, 0,2.726524,-5.700108,-0.450337 \backslash \mathrm{H}, 0,2.7839$ $87,-6.793202,-0.418634 \backslash \mathrm{C}, 0,1.929975,-5.025612,0.485561 \backslash \mathrm{H}, 0,1.348163,-5$ $.58391,1.22388|C, 0,3.608613,0.996298,-0.467499| C, 0,4.261855,0.496822,-$ $1.625299|C, 0,4.009699,0.554009,0.823726| C, 0,5.356848,-0.374327,-1.4658$ $18 \mathrm{IC}, 0,5.107279,-0.319659,0.928888 \mathrm{IC}, 0,5.813982,-0.773933,-0.198342 \mathrm{IH}$, $0,5.8714,-0.743255,-2.360479 \mathrm{H}, 0,5.44029,-0.6468,1.92042 \mathrm{CC}, 0,-3.933185$ $,-0.156989,-0.907011 \backslash \mathrm{C}, 0,-4.239758,-1.388636,-0.262772 \backslash \mathrm{C}, 0,-4.298089,0$ $.042403,-2.265475 \backslash \mathrm{C}, 0,-4.935155,-2.376646,-0.982545 \backslash \mathrm{C}, 0,-4.984536,-0.9$ $8249,-2.944037 \backslash \mathrm{C}, 0,-5.319229,-2.196498,-2.323207 \backslash \mathrm{H}, 0,-5.174107,-3.3224$ $21,-0.48274 \backslash \mathrm{H}, 0,-5.27192,-0.82947,-3.991676 \backslash \mathrm{H}, 0,4.044648,-5.515525,-2$. $164138 \mathrm{IC}, 0,-2.943499,0.534367,5.261085 \mathrm{H}, 0,-3.83936,0.626133,4.618619$ $\mathrm{H}, 0,-3.260319,0.142854,6.241627 \backslash \mathrm{H}, 0,-2.545488,1.555599,5.39786 \mathrm{IC}, 0,-3$. $991988,1.344923,-3.007032 \backslash \mathrm{H}, 0,-3.435169,2.003831,-2.319846 \backslash \mathrm{C}, 0,-3.8191$ $9,-1.674398,1.180342 \mathrm{CC}, 0,-6.062029,-3.28908,-3.087411 \backslash \mathrm{H}, 0,-6.250925,-2$ $.900513,-4.105952 \backslash \mathrm{C}, 0,-5.019746,-1.589226,2.145714 \backslash \mathrm{H}, 0,-4.695944,-1.77$ 
$0254,3.187218 \mathrm{H}, 0,-5.504283,-0.598068,2.104829 \backslash \mathrm{H}, 0,-5.783543,-2.34802$, 1.892152\C,0,-7.42873,-3.607695,-2.447125\H,0,-7.306769,-4.023614,-1.4 29838\H,0,-8.05612,-2.701652,-2.369994\H,0,-7.977034,-4.353934,-3.0511 $31 \backslash \mathrm{C}, 0,-5.204544,-4.564409,-3.224402 \backslash \mathrm{H}, 0,-5.727833,-5.322842,-3.835425$ $\mathrm{IH}, 0,-4.23493,-4.344246,-3.705733 \mathrm{|H}, 0,-4.997827,-5.012938,-2.235116 \backslash \mathrm{C}$, $0,-3.093255,1.104761,-4.237228 \mathrm{H}, 0,-3.605615,0.488957,-4.999355 \backslash \mathrm{H}, 0,-2$ $.82519,2.067535,-4.710406 \mathrm{H}, 0,-2.160253,0.594503,-3.944517 \backslash \mathrm{C}, 0,-5.2897$ $81,2.086871,-3.388688 \mathrm{H}, 0,-5.056873,3.052718,-3.873738 \mathrm{H}, 0,-5.901101,1$ $.494229,-4.093986 \mathrm{H}, 0,-5.906618,2.292568,-2.495118 \mathrm{IC}, 0,7.041335,-1.668$ $246,-0.036983 \mathrm{IH}, 0,7.234058,-1.75515,1.049069 \mathrm{IC}, 0,3.814415,0.898727,-3$. $032118 \mathrm{IH}, 0,2.830559,1.389221,-2.935002 \backslash \mathrm{C}, 0,3.306341,1.061221,2.08535 \mathrm{IH}$ $, 0,2.261591,1.28576,1.810874 \backslash \mathrm{C}, 0,6.796689,-3.090636,-0.579221 \backslash \mathrm{H}, 0,7.68$ $6189,-3.726673,-0.415904 \backslash \mathrm{H}, 0,5.937788,-3.566892,-0.075904 \backslash \mathrm{H}, 0,6.588922$ $,-3.073458,-1.66478 \mathrm{IC}, 0,8.290385,-1.035431,-0.683541 \mathrm{lH}, 0,8.174781,-0.9$ $51765,-1.779765 \mathrm{IH}, 0,8.478202,-0.022687,-0.28394 \backslash \mathrm{H}, 0,9.185737,-1.653413$ ,-0.487299IC, $0,3.257473,0.020442,3.218168 \mathrm{IH}, 0,2.879269,-0.950454,2.854$ $858 \mathrm{H}, 0,4.252618,-0.148022,3.668367 \mathrm{H}, 0,2.586867,0.3696,4.023263 \mathrm{IC}, 0,3$ $.944829,2.377967,2.577633 \mathrm{lH}, 0,3.921031,3.151992,1.790715 \mathrm{H}, 0,3.402426$ 2.768073,3.458622\Н,0,4.999877,2.216261,2.866273\C,0,4.79577,1.912476, $-3.656813 \mathrm{H}, 0,4.901606,2.813288,-3.027145 \backslash \mathrm{H}, 0,5.800162,1.465412,-3.778$ $703 \mathrm{\backslash H}, 0,4.443054,2.232752,-4.654588 \mathrm{IC}, 0,3.616055,-0.321392,-3.9526 \mathrm{IH}, 0$

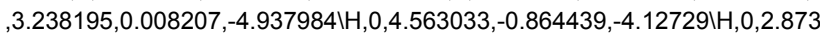
$659,-1.011813,-3.520984 \backslash \mathrm{C}, 0,-3.098062,-3.029848,1.322043 \mathrm{H}, 0,-3.776786$ $,-3.879828,1.123778 \mathrm{H}, 0,-2.245072,-3.086189,0.626422 \mathrm{H}, 0,-2.715084,-3$ 14706,2.351861।H, $0,-3.096704,-0.894935,1.472031$ IIVersion=ES64L-G09RevD 01IState=1-AlHF=-3216.0960396 \RMSD=2.058e-09\Dipole=1.0498946,-2.3514 819,5.2624789lQuadrupole=-9.5013742,13.4643226,-3.9629484,-13.5367305, $-6.4079337,-15.0884697 \backslash \mathrm{PG}=\mathrm{C} 01$ [X(C65H72N1O4P1)]॥@

Conf3

1\1\GINC-WORKER2ISPIRTPSSTPSSIdef2SVPIC65H72N1O4P1\JHIOEI06-May-201910 II\#p tpsstpss/def2svp int=ultrafine empiricaldispersion=gd3 scrf=(smd, solvent $=$ ch2cl2, read,externaliteration, dovacuum) Ititlell0,1 $\backslash \mathrm{P}, 0,-0.090$ $393,-0.541341,-1.174492 \backslash 0,0,-1.350103,0.526771,-1.454139 \backslash 0,0,0.836758$, $0.400252,-0.10387810,0,0.584213,-0.744615,-2.498014 \backslash 0,0,-0.548967,-1.6$ $78426,-0.266704 \mathrm{IC}, 0,-1.263845,2.268832,0.181636 \mathrm{IC}, 0,-1.942186,1.19706$, $-0.404913|\mathrm{C}, 0,-3.267009,0.808618,-0.031728| \mathrm{C}, 0,-3.891736,1.528175,0.97$ $8073 \mathrm{IH}, 0,-4.917402,1.274986,1.265318 \mid \mathrm{C}, 0,-3.223964,2.573342,1.678608 \mathrm{IC}$ $, 0,-1.880435,2.939244,1.300527 \backslash \mathrm{C}, 0,-1.201052,3.916347,2.089683 \mathrm{H}, 0,-0$ $165787,4.172117,1.847347 \backslash \mathrm{C}, 0,-1.825543,4.52711,3.167591 \backslash \mathrm{H}, 0,-1.278623$ $5.266249,3.76327 \backslash \mathrm{C}, 0,-3.164424,4.19731,3.509972 \mathrm{H}, 0,-3.648308,4.690872$ ,4.359759।C, $0,-3.844972,3.235422,2.780192 \mathrm{H}, 0,-4.868532,2.951338,3.049$ $776 \mathrm{IC}, 0,0.078615,2.667111,-0.330283 \mathrm{IC}, 0,1.105922,1.719156,-0.405246 \mathrm{IC}$, $0,2.462225,2.067911,-0.689656 \backslash \mathrm{C}, 0,2.743837,3.391516,-1.001459 \backslash \mathrm{H}, 0,3.77$ $8809,3.688438,-1.200747 \backslash \mathrm{C}, 0,1.718256,4.376559,-1.085267 \backslash \mathrm{C}, 0,0.36228,4$. $017047,-0.750645 \backslash \mathrm{C}, 0,-0.652987,5.012094,-0.886043 \mathrm{IH}, 0,-1.692441,4.7449$ $43,-0.674527 \backslash \mathrm{C}, 0,-0.342615,6.299489,-1.299041 \mathrm{WH}, 0,-1.140629,7.042724,-$ $1.404642 \mathrm{IC}, 0,0.999586,6.66072,-1.593823 \mathrm{IH}, 0,1.23242,7.682644,-1.912531$ IC, $0,2.006985,5.714345,-1.491409|\mathrm{H}, 0,3.043903,5.974773,-1.732839| \mathrm{C}, 0,-$ $1.762359,-0.333249,4.717655 \backslash \mathrm{C}, 0,-1.343062,-1.555447,5.284581 \backslash \mathrm{H}, 0,-1.76$ $5546,-1.879488,6.24214 \backslash \mathrm{C}, 0,-0.395245,-2.361632,4.646254 \mathrm{H}, 0,-0.095295$, $-3.306132,5.109864 \backslash \mathrm{C}, 0,0.135683,-1.983554,3.390664 \backslash \mathrm{C}, 0,-0.272199,-0.75$ $2068,2.82254 \mathrm{IH}, 0,0.151361,-0.410648,1.874398 \mathrm{IC}, 0,-1.19352,0.056438,3.4$ $85071 \mathrm{WH}, 0,-1.48287,1.010085,3.034723 \backslash \mathrm{C}, 0,1.101822,-2.852306,2.702727 \backslash \mathrm{C}$ $, 0,2.031586,-3.714957,3.501162 \backslash \mathrm{H}, 0,2.937013,-3.967451,2.928421 \backslash \mathrm{H}, 0,1.5$ $26347,-4.659247,3.775681 \backslash \mathrm{H}, 0,2.310341,-3.200263,4.433238 \mathrm{~N}, 0,1.14428$,$2.821242,1.389902 \backslash \mathrm{H}, 0,0.426483,-2.234905,0.831551 \backslash \mathrm{C}, 0,2.00202,-3.55293$ $6,0.508707 \backslash \mathrm{C}, 0,2.642569,-2.830492,-0.515509 \backslash \mathrm{H}, 0,2.548722,-1.741904,-0$. $563241 \mathrm{lC}, 0,3.417927,-3.518772,-1.45581 \mathrm{H}, 0,3.919458,-2.955712,-2.24782$ 3IC, $0,3.55614,-4.913839,-1.377821 \backslash \mathrm{C}, 0,2.910595,-5.626188,-0.355068 \mathrm{WH}, 0$ ,3.004738,-6.715642,-0.297006।C,0,2.123765,-4.951909,0.589082।H, $0,1.58$ $4898,-5.507435,1.361319 \mid C, 0,3.563628,1.055417,-0.578293 \backslash \mathrm{C}, 0,4.191929,0$ $.542321,-1.743659 \backslash \mathrm{C}, 0,4.018945,0.660739,0.710352 \backslash \mathrm{C}, 0,5.316326,-0.29324$ $4,-1.597693 \backslash \mathrm{C}, 0,5.144645,-0.177808,0.802031 \backslash \mathrm{C}, 0,5.826595,-0.643574,-0$ $33592 \backslash \mathrm{H}, 0,5.811885,-0.671926,-2.499058 \backslash \mathrm{H}, 0,5.519143,-0.467672,1.790306$ $\mid \mathrm{C}, 0,-3.954991,-0.322138,-0.734553 \backslash \mathrm{C}, 0,-4.21732,-1.538027,-0.046037 \backslash \mathrm{C}$, $0,-4.36713,-0.169834,-2.087684 \backslash \mathrm{C}, 0,-4.924413,-2.55639,-0.714403 \backslash \mathrm{C}, 0,-5$ $.060202,-1.221271,-2.712287 \backslash \mathrm{C}, 0,-5.359786,-2.420721,-2.042182 \backslash \mathrm{H}, 0,-5.1$ $36368,-3.49367,-0.185753 \mathrm{H}, 0,-5.381105,-1.095826,-3.753114 \mathrm{IH}, 0,4.16505$ $1,-5.445613,-2.11647 \backslash \mathrm{C}, 0,-2.797231,0.536664,5.385513 \backslash \mathrm{H}, 0,-3.722285,0.5$ $64081,4.779294 \backslash \mathrm{H}, 0,-3.055091,0.166539,6.391319 \mathrm{H}, 0,-2.442137,1.579078$ $5.470161 \backslash \mathrm{C}, 0,-4.104441,1.112656,-2.879673 \backslash \mathrm{H}, 0,-3.533579,1.801353,-2.23$ $4774|\mathrm{C}, 0,-3.737665,-1.77886,1.386639| \mathrm{C}, 0,-6.116747,-3.548967,-2.737656$ $\mathrm{IH}, 0,-6.264066,-4.349022,-1.987874 \mathrm{CC}, 0,-4.901059,-1.697252,2.396646 \mathrm{lH}$, $0,-4.531413,-1.847277,3.427728 \mathrm{H}, 0,-5.407726,-0.717364,2.35355 \mathrm{H}, 0,-5$. $657863,-2.477592,2.192205 \backslash \mathrm{C}, 0,-5.29617,-4.144183,-3.900844 \backslash \mathrm{H}, 0,-5.1266$ 
4,-3.392854,-4.694167\H, $0,-4.309163,-4.497647,-3.552858 \backslash \mathrm{H}, 0,-5.827996$, $-5.000431,-4.355336 \mathrm{IC}, 0,-7.510107,-3.094349,-3.218123 \mathrm{IH}, 0,-8.065225,-3$ $.941874,-3.660553 \backslash \mathrm{H}, 0,-8.109496,-2.690614,-2.382487 \backslash \mathrm{H}, 0,-7.430836,-2.3$ $06896,-3.990304 \backslash \mathrm{C}, 0,-3.244823,0.841921,-4.131346 \mathrm{IH}, 0,-3.772722,0.18988$ $8,-4.851645 \mathrm{\backslash H}, 0,-3.00992,1.790372,-4.64894 \mathrm{IH}, 0,-2.293696,0.35761,-3.85$ $3145 \backslash \mathrm{C}, 0,-5.424222,1.8252,-3.241049 \mathrm{H}, 0,-5.220923,2.778282,-3.763266 \mathrm{IH}$ $, 0,-6.049952,1.20279,-3.907052 \backslash \mathrm{H}, 0,-6.014662,2.050806,-2.334535 \backslash \mathrm{C}, 0,7$. $0867,-1.494518,-0.191278 \mathrm{H}, 0,7.31354,-1.549551,0.890279 \mid \mathrm{C}, 0,3.686265,0$ 893402,-3.144462) $\mathrm{H}, 0,2.695478,1.364794,-3.026066 \backslash \mathrm{C}, 0,3.33999,1.183056$ $, 1.979426 \mathrm{IH}, 0,2.27892,1.359501,1.733968 \mathrm{IC}, 0,6.874788,-2.936232,-0.6945$ $56 \mathrm{H}, 0,7.789489,-3.538451,-0.542773 \mathrm{H}, 0,6.046509,-3.428887,-0.157116 \mathrm{HH}$ $, 0,6.636819,-2.950855,-1.773916 \mid \mathrm{C}, 0,8.295183,-0.836922,-0.888572 \backslash \mathrm{H}, 0,8$ $.144643,-0.782521,-1.98241 \mathrm{H}, 0,8.461818,0.19017,-0.51715 \backslash \mathrm{H}, 0,9.215266$, $-1.421862,-0.705879 \backslash \mathrm{C}, 0,3.3668,0.180294,3.146894 \backslash \mathrm{H}, 0,3.004676,-0.81319$ $1,2.831554 \backslash \mathrm{H}, 0,4.38287,0.05646,3.563701 \backslash \mathrm{H}, 0,2.716708,0.5373,3.965261 \backslash \mathrm{C}$ $, 0,3.942962,2.53816,2.408664 \mathrm{H}, 0,3.866591,3.284781,1.598929 \mathrm{H}, 0,3.4125$ $66,2.936519,3.293234 \backslash \mathrm{H}, 0,5.011556,2.42547,2.669511 \backslash \mathrm{C}, 0,4.620256,1.9105$ $94,-3.83253 \backslash \mathrm{H}, 0,4.728251,2.830525,-3.231306 \backslash \mathrm{H}, 0,5.629587,1.482889,-3.9$ $80399 \backslash \mathrm{H}, 0,4.223281,2.195326,-4.824435 \backslash \mathrm{C}, 0,3.483026,-0.356833,-4.0224 \backslash \mathrm{H}$ $, 0,3.068507,-0.063449,-5.004492 \mathrm{H}, 0,4.434445,-0.887215,-4.211203 \backslash \mathrm{H}, 0,2$ $.767559,-1.046755,-3.547146 \mathrm{IC}, 0,-2.978977,-3.113494,1.530161 \backslash \mathrm{H}, 0,-3.64$ $377,-3.98331,1.375537 \backslash \mathrm{H}, 0,-2.151644,-3.164486,0.803847 \backslash \mathrm{H}, 0,-2.554883,-$ 3.198084,2.54712\H,0,-3.022821,-0.976835,1.631844IIVersion=ES64L-G09Re vD.01/State=1-AlHF=-3216.0960294IRMSD=2.052e-09lDipole=1.3180727,-2.12 $30292,5.2663943 \backslash$ Quadrupole $=-8.9319945,11.2411187,-2.3091242,-14.992139$ 8,-5.0747244,-15.696795IPG=C01 [X(C65H72N1O4P1)]॥@

\section{TRIP/1E Type III.1}

1I1IGINC-WORKER1ISPIRTPSSTPSSIdef2SVPIC65H72N1O4P1\JHIOEI27-Mar-201910 II\# tpsstpss/def2svp int=ultrafine empiricaldispersion=gd3 scrf $=(\mathrm{smd}$, solvent $=$ ch2cl2, read,externaliteration, dovacuum) $\mid(t i t l e l \mid 0,1 \backslash \mathrm{H}, 0,-5.712$ $31,0.551506,-2.0845 \backslash C, 0,-5.217347,0.454445,-1.110205 \backslash C, 0,-4.140798,1.3$ $05227,-0.80799 \backslash \mathrm{C}, 0,-5.703145,-0.49898,-0.195541 \backslash \mathrm{C}, 0,-3.503063,1.185282$ $, 0.457582 \backslash \mathrm{C}, 0,-3.725315,2.370052,-1.825077 \backslash \mathrm{C}, 0,-5.030883,-0.626222,1.0$ $29857 \backslash \mathrm{C}, 0,-6.953155,-1.306385,-0.545022 \backslash \mathrm{C}, 0,-2.42593,2.147774,0.85695$ C,0,-3.930427,0.184386,1.371492\C,0,-4.860968,3.387137,-2.062421।C,0,$3.243194,1.746094,-3.149402 \backslash \mathrm{H}, 0,-2.875635,2.930265,-1.400273 \backslash \mathrm{H}, 0,-5.17$ $4929,3.86192,-1.115281 \backslash \mathrm{H}, 0,-5.748612,2.903864,-2.510859 \backslash \mathrm{H}, 0,-4.528172$, $4.185379,-2.751306 \backslash \mathrm{H}, 0,-2.89849,2.536293,-3.841821 \backslash \mathrm{H}, 0,-4.055396,1.190$ $618,-3.653172 \mathrm{H}, 0,-2.402716,1.055504,-2.970615 \backslash \mathrm{H}, 0,-5.366268,-1.37636$, $1.75267 \backslash \mathrm{C}, 0,-8.201823,-0.395391,-0.504575 \backslash \mathrm{C}, 0,-7.168786,-2.549115,0.33$ $2149 \backslash \mathrm{H}, 0,-6.827527,-1.64689,-1.590917 \backslash \mathrm{H}, 0,-8.090667,0.471759,-1.179232$ $\mathrm{IH}, 0,-8.368168,-0.01002,0.518567 \backslash \mathrm{H}, 0,-9.105163,-0.954128,-0.811629 \mathrm{H}, 0$ ,-7.416856,-2.272411,1.373458\H,0,-6.273674,-3.194138,0.356186\H,0,-8. $010507,-3.147846,-0.05939 \backslash \mathrm{C}, 0,-1.137088,2.130885,0.237809 \backslash \mathrm{C}, 0,-2.65269$ $4,3.093675,1.849275 \backslash C, 0,-3.242342,-0.042412,2.720695 \backslash O, 0,-0.927382,1.2$ $4762,-0.802506 \mathrm{IC}, 0,-0.10798,3.008405,0.59368 \mathrm{IH}, 0,-3.640855,3.153937,2$. $316966 \backslash \mathrm{C}, 0,-1.626806,3.968707,2.306727 \backslash \mathrm{C}, 0,-2.83326,-1.515782,2.922745$ IC, $0,-4.124488,0.444851,3.889406 \backslash \mathrm{H}, 0,-2.313553,0.552578,2.725203 \backslash \mathrm{H}, 0,-$ $2.270968,-1.626423,3.86844 \backslash \mathrm{H}, 0,-2.185669,-1.849489,2.097376 \backslash \mathrm{H}, 0,-3.712$ $786,-2.183394,2.979935 \mathrm{H}, 0,-3.600558,0.312914,4.854166 \mathrm{H}, 0,-4.383492,1$ $.513091,3.785832 \backslash \mathrm{H}, 0,-5.069366,-0.128315,3.936357 \backslash \mathrm{P}, 0,0.024963,-0.1127$ $46,-0.558885 \backslash \mathrm{C}, 0,1.18566,2.95481,-0.143659 \backslash \mathrm{C}, 0,-0.321382,3.912207,1.69$ $657 \backslash C, 0,-1.859456,4.882297,3.37906210,0,-0.637989,-1.043995,0.4501910$, $0,0.442926,-0.593453,-1.923892 \backslash O, 0,1.291191,0.597333,0.290182 \backslash C, 0,1.86$ $9025,1.737737,-0.238549 \mid \mathrm{C}, 0,1.766758,4.119052,-0.764902 \mathrm{CC}, 0,0.711303,4$ $.737011,2.23804 \mathrm{IH}, 0,-2.860187,4.920213,3.824412 \mathrm{lC}, 0,-0.841782,5.690978$ ,3.859415।C,0,3.183948,1.626376,-0.78989।C,0,1.076792,5.365092,-0.8697 $3 \backslash \mathrm{C}, 0,3.087997,4.021611,-1.334318 \mathrm{H}, 0,1.719887,4.67292,1.820215 \backslash \mathrm{C}, 0,0$. $457467,5.603986,3.290717 \backslash \mathrm{H}, 0,-1.030564,6.384838,4.685912 \backslash \mathrm{C}, 0,3.775632$, $2.774443,-1.29904 \backslash \mathrm{C}, 0,3.917514,0.319321,-0.751717 \backslash \mathrm{H}, 0,0.057407,5.44422$ $,-0.480791 \backslash \mathrm{C}, 0,1.673064,6.465077,-1.468648 \mid \mathrm{C}, 0,3.679461,5.176415,-1.93$ $01 \mathrm{H}, 0,1.268285,6.22248,3.691318 \mathrm{H}, 0,4.795324,2.724908,-1.694251 \mathrm{CC}, 0,4$ $.463858,-0.134912,0.479888 \mathrm{IC}, 0,4.097262,-0.443371,-1.936127 \mathrm{VH}, 0,1.1205$ $83,7.408177,-1.544071 \backslash \mathrm{C}, 0,2.99088,6.377503,-1.992591 \backslash \mathrm{H}, 0,4.688617,5.08$ $904,-2.348807 \backslash \mathrm{C}, 0,5.19731,-1.335516,0.499726 \backslash \mathrm{C}, 0,4.307139,0.670098,1.7$ $72286 \mid C, 0,4.852842,-1.629645,-1.865876 \backslash C, 0,3.507492,0.000032,-3.276822$ $\mathrm{IH}, 0,3.453025,7.255511,-2.456998 \mathrm{H}, 0,5.62096,-1.679258,1.450826 \mathrm{IC}, 0,5$. $41594,-2.093282,-0.665503 \backslash \mathrm{C}, 0,5.6759,1.131322,2.31387 \backslash \mathrm{C}, 0,3.508777,-0$. $099487,2.842554 \backslash \mathrm{H}, 0,3.732543,1.580887,1.53345 \backslash \mathrm{H}, 0,5.54296,1.762643,3.2$ $11557 \backslash \mathrm{H}, 0,6.224673,1.721477,1.5579 \mathrm{H}, 0,6.309492,0.270971,2.598242 \backslash \mathrm{H}, 0$, $3.361238,0.532416,3.737787 \mathrm{H}, 0,4.039556,-1.014128,3.163075 \mathrm{H}, 0,2.51655$ $1,-0.388831,2.458192 \backslash \mathrm{H}, 0,5.01102,-2.217028,-2.778191 \backslash \mathrm{C}, 0,4.580317,0.66$ $8824,-4.162686 \mathrm{IC}, 0,2.825261,-1.15497,-4.035107 \backslash \mathrm{H}, 0,2.72548,0.746581,-3$ $.056585 \mathrm{H}, 0,4.137943,1.013369,-5.115793 \mathrm{H}, 0,5.037698,1.541057,-3.66456$ $7 \backslash \mathrm{H}, 0,5.390151,-0.045491,-4.40261 \backslash \mathrm{H}, 0,2.309686,-0.761962,-4.9307 \backslash \mathrm{H}, 0,2$ $.072663,-1.643925,-3.396469 \backslash \mathrm{H}, 0,3.555145,-1.909563,-4.381889 \backslash \mathrm{C}, 0,6.232$ 
$864,-3.383248,-0.642177 \backslash \mathrm{C}, 0,7.643648,-3.163895,-0.059771 \backslash \mathrm{C}, 0,5.494758$ $-4.511189,0.107631 \backslash \mathrm{H}, 0,6.350503,-3.704853,-1.69421 \backslash \mathrm{H}, 0,7.598879,-2.879$ $524,1.007736 \mathrm{H}, 0,8.18077,-2.365061,-0.601885 \mathrm{H}, 0,8.241614,-4.090645,-0$ $133315 \backslash \mathrm{H}, 0,5.35467,-4.2542,1.172832 \backslash \mathrm{H}, 0,6.073209,-5.452389,0.060903 \backslash \mathrm{H}$ $, 0,4.498449,-4.698239,-0.32989 \backslash \mathrm{H}, 0,-5.368708,-2.688743,-3.968513 \backslash \mathrm{C}, 0,-$ $4.574688,-2.844753,-3.230768 \backslash \mathrm{C}, 0,-3.364051,-2.142045,-3.33877 \backslash \mathrm{C}, 0,-4.7$ $73963,-3.732776,-2.162535 \backslash \mathrm{H}, 0,-3.207999,-1.435232,-4.159728 \backslash \mathrm{C}, 0,-2.345$ $334,-2.336144,-2.397979|\mathrm{H}, 0,-5.727193,-4.26002,-2.055216| \mathrm{C}, 0,-3.766704$ $,-3.934937,-1.209398 \mathrm{H}, 0,-1.394618,-1.795554,-2.473675 \backslash \mathrm{C}, 0,-2.543217,-$ $3.252589,-1.346806 \mathrm{WH}, 0,-3.944203,-4.591086,-0.354196 \mathrm{WN}, 0,-1.468529,-3$ $408828,-0.416791 \backslash \mathrm{C}, 0,-0.98909,-4.517979,0.101815 \backslash \mathrm{H}, 0,-1.04031,-2.46484$ $8,-0.095288 \mid C, 0,-1.566988,-5.867845,-0.198602 \backslash C, 0,0.178772,-4.416968,0$ $.991379 \backslash \mathrm{H}, 0,-2.324491,-6.132302,0.562205 \backslash \mathrm{H}, 0,-2.058468,-5.886202,-1.18$ $386 \mathrm{WH}, 0,-0.773756,-6.629507,-0.163137 \backslash \mathrm{C}, 0,0.299726,-5.29013,2.099502 \backslash \mathrm{C}$ $, 0,1.206082,-3.475074,0.750955 \backslash \mathrm{H}, 0,-0.477895,-6.033995,2.298488 \mathrm{IC}, 0,1$. $387444,-5.181516,2.967819 \backslash \mathrm{H}, 0,1.171075,-2.834609,-0.133895 \backslash \mathrm{C}, 0,2.30350$ $2,-3.397637,1.610735 \backslash \mathrm{H}, 0,1.450188,-5.847668,3.835617 \backslash \mathrm{C}, 0,2.407197,-4.2$ $29581,2.745069 \backslash \mathrm{H}, 0,3.098599,-2.680581,1.389268 \mathrm{lC}, 0,3.565382,-4.10036,3$ $.703011 \backslash \mathrm{H}, 0,3.91352,-5.088827,4.050079 \backslash \mathrm{H}, 0,3.255882,-3.530575,4.600165$ $\mathrm{IH}, 0,4.412804,-3.565968,3.244565||$ Version=ES64L-G09RevD.01 $\mid$ State=1-AlH $\mathrm{F}=-3216.0898631 \backslash \mathrm{RMSD}=5.297 \mathrm{e}-09 \mathrm{|Dipole}=-1.2847597,-4.7048372,1.2564765 \backslash$ Quadrupole $=-5.0187217,26.9891532,-21.9704314,14.9074858,11.1982848,-7$. 1062127\PG=C01 [X(C65H72N1O4P1)]॥@

\section{TRIP/1E Type III.2}

1।1\GINC-WORKER2ISPIRTPSSTPSSIdef2SVPIC65H72N1O4P1\JHIOE\27-Mar-201910

II\#p tpsstpss/def2svp int=ultrafine empiricaldispersion=gd3 scrf=(smd, solvent $=$ ch2cl2, read,externaliteration, dovacuum) \titlel|0,1\P,0,0.1771 $26,-0.15514,-0.393091 \backslash 0,0,-0.60123,1.287684,-0.753639 \backslash 0,0,1.508061,0.4$ $672,0.424385 \backslash \mathrm{O}, 0,0.540274,-0.785479,-1.710831 \backslash \mathrm{O}, 0,-0.597575,-0.909954$, $0.68035 \backslash \mathrm{C}, 0,0.412979,3.042288,0.519768 \mid \mathrm{C}, 0,-0.709295,2.264679,0.215221$ IC, $0,-1.992989,2.476696,0.808751 \backslash \mathrm{C}, 0,-2.120315,3.519287,1.717083 \backslash \mathrm{H}, 0,-$ 3.1006,3.736591,2.153462lC,0,-1.004065,4.307122,2.120215।C,0,0.292832, $4.051997,1.542681 \mathrm{IC}, 0,1.407269,4.797432,2.035136 \mathrm{IH}, 0,2.406299,4.584585$ , 1.644291\C,0,1.24312,5.774,3.006635\H,0,2.115023,6.328554,3.370867\C, $0,-0.043506,6.056097,3.53898 \mathrm{H}, 0,-0.160304,6.834836,4.300557 \backslash \mathrm{C}, 0,-1.14$ $1657,5.329168,3.107756 \mathrm{H}, 0,-2.135958,5.518492,3.528317 \backslash \mathrm{C}, 0,1.699663,2$. $777864,-0.185401 \backslash C, 0,2.226499,1.482036,-0.177438 \mid C, 0,3.519449,1.159949$ $-0.696914 \mathrm{IC}, 0,4.257224,2.181195,-1.279331 \mathrm{H}, 0,5.263705,1.973373,-1.65$ 6693IC, $0,3.732498,3.498837,-1.41974 I C, 0,2.429167,3.807377,-0.883509 I C$, $0,1.904896,5.118114,-1.097955 \mathrm{VH}, 0,0.901657,5.357225,-0.733689 \mathrm{CC}, 0,2.64$ $0261,6.08286,-1.770959 \mathrm{H}, 0,2.212132,7.078962,-1.928479 \mathrm{IC}, 0,3.939626,5$. $788068,-2.2639 \backslash \mathrm{H}, 0,4.512868,6.560453,-2.788245 \backslash \mathrm{C}, 0,4.469368,4.518622,-$ $2.094425 \mathrm{IH}, 0,5.462111,4.27148,-2.487562 \mathrm{IC}, 0,4.052042,-0.234865,-0.5597$ $01 \backslash \mathrm{C}, 0,4.141199,-1.089865,-1.69361 \backslash \mathrm{C}, 0,4.468258,-0.699937,0.716127 \backslash \mathrm{C}, 0$ $, 4.662515,-2.385349,-1.525379 \backslash \mathrm{C}, 0,4.986744,-2.003875,0.829936 \backslash \mathrm{C}, 0,5.09$ $3903,-2.863767,-0.274878 \mathrm{H}, 0,4.734731,-3.042906,-2.39901 \mathrm{H}, 0,5.318625$, $-2.362151,1.812139 \backslash \mathrm{C}, 0,-3.164795,1.614547,0.444378 \mid \mathrm{C}, 0,-3.674233,0.672$ $06,1.377699 \backslash \mathrm{C}, 0,-3.790636,1.765123,-0.823223 \backslash \mathrm{C}, 0,-4.832768,-0.057569,1$ $.045406 \mathrm{IC}, 0,-4.933127,0.99986,-1.112715 \backslash \mathrm{C}, 0,-5.486858,0.09565,-0.18721$ $\mathrm{IH}, 0,-5.227119,-0.76856,1.777248 \backslash \mathrm{H}, 0,-5.419145,1.120549,-2.088705 \backslash \mathrm{C}, 0$, $-3.273777,2.759519,-1.864496 \backslash \mathrm{H}, 0,-2.403322,3.279166,-1.429152 \mathrm{IC}, 0,-3.0$ $07677,0.433343,2.735563 \mid \mathrm{C}, 0,-6.777508,-0.644102,-0.540031 \mathrm{H}, 0,-6.66175$ $3,-0.999226,-1.581967 \backslash \mathrm{C}, 0,-3.761402,1.164384,3.867484 \backslash \mathrm{H}, 0,-3.822027,2$. $250578,3.684543 \mathrm{H}, 0,-4.794382,0.779886,3.961037 \mathrm{VH}, 0,-3.252161,1.009994$ ,4.836863।C,0,-7.977157,0.330447,-0.519255।H,0,-7.811384,1.18772,-1.19 $5344 \backslash \mathrm{H}, 0,-8.903918,-0.181964,-0.83701 \backslash \mathrm{H}, 0,-8.137179,0.728496,0.500016 \backslash$ $\mathrm{C}, 0,-7.063986,-1.869207,0.341518 \mathrm{H}, 0,-7.298599,-1.576178,1.38148 \mathrm{H}, 0,-$ $7.936963,-2.421441,-0.050298 \backslash \mathrm{H}, 0,-6.206056,-2.563067,0.366904 \backslash \mathrm{C}, 0,-2.7$ $8735,2.048744,-3.14346 \mathrm{H}, 0,-1.99545,1.320613,-2.902973 \mathrm{H}, 0,-3.614526,1$ $.517739,-3.649607 \backslash \mathrm{H}, 0,-2.373838,2.78424,-3.858181 \backslash \mathrm{C}, 0,-4.328912,3.8382$ $12,-2.184975 \mathrm{lH}, 0,-3.915201,4.589205,-2.882988 \mathrm{H}, 0,-5.22613,3.398611,-2$ $.658517 \backslash \mathrm{H}, 0,-4.651896,4.364099,-1.268281 \backslash \mathrm{C}, 0,5.657463,-4.273914,-0.120$ $181 \backslash \mathrm{H}, 0,5.905676,-4.407796,0.949715 \backslash \mathrm{C}, 0,3.705355,-0.628592,-3.086148 \mathrm{IH}$ $, 0,3.069521,0.263147,-2.95088 \backslash \mathrm{C}, 0,4.385067,0.179211,1.96528 \mathrm{H}, 0,3.9483$ $33,1.14762,1.668081 \backslash \mathrm{C}, 0,4.618019,-5.348931,-0.499383 \backslash \mathrm{H}, 0,4.344002,-5.2$ $79375,-1.568266 \mathrm{lH}, 0,5.02316,-6.36223,-0.321929 \mathrm{H}, 0,3.693482,-5.240023$, $0.094458 \backslash \mathrm{C}, 0,6.958264,-4.453834,-0.929347 \mathrm{VH}, 0,7.716813,-3.70536,-0.638$ $293 \backslash \mathrm{H}, 0,7.386646,-5.459387,-0.763033 \backslash \mathrm{H}, 0,6.770703,-4.343125,-2.013344 \backslash$ $\mathrm{C}, 0,3.455278,-0.432799,3.032859 \mathrm{H}, 0,3.341773,0.260775,3.886471 \mathrm{H}, 0,2.4$ $54289,-0.627922,2.612932 \backslash \mathrm{H}, 0,3.8607,-1.383164,3.425658 \backslash \mathrm{C}, 0,5.786476,0$. $475432,2.537658 \mathrm{H}, 0,6.434547,0.947144,1.777093 \mathrm{H}, 0,5.715324,1.163426,3$ $40019 \backslash \mathrm{H}, 0,6.285545,-0.448575,2.883756 \backslash \mathrm{C}, 0,4.929709,-0.222418,-3.93472$ $2 \mathrm{H}, 0,5.592336,-1.091177,-4.107108 \mathrm{H}, 0,4.610399,0.162757,-4.920841 \mathrm{H}, 0$ $, 5.527283,0.561941,-3.43878 \mathrm{IC}, 0,2.854756,-1.679631,-3.825247 \backslash \mathrm{H}, 0,2.481$ $332,-1.258712,-4.777133 \backslash \mathrm{H}, 0,3.439125,-2.584338,-4.074967 \backslash \mathrm{H}, 0,1.984884$, 
$-1.966465,-3.213103 \backslash \mathrm{C}, 0,-2.866788,-1.065159,3.070543 \backslash \mathrm{H}, 0,-2.252765,-1$ $193801,3.980628 \mathrm{H}, 0,-3.846473,-1.539208,3.265339 \backslash \mathrm{H}, 0,-2.373321,-1.6018$ $01,2.24719 \mathrm{H}, 0,-1.987271,0.849396,2.676156 \mid \mathrm{C}, 0,-4.394121,-2.324041,-2$. 986389|C,0,-3.072501,-1.897479,-2.728531\H,0,-2.634093,-1.097533,-3.33 $3736 \backslash \mathrm{C}, 0,-2.304893,-2.478803,-1.720937 \backslash \mathrm{H}, 0,-1.271177,-2.142405,-1.5911$ $88 \mathrm{IC}, 0,-2.837648,-3.526686,-0.929372 \mathrm{IC}, 0,-4.15174,-3.977809,-1.20025 \mathrm{H}$ $, 0,-4.594004,-4.78209,-0.60497 \backslash \mathrm{C}, 0,-4.915732,-3.374266,-2.203159 \backslash \mathrm{H}, 0,-$ $5.940741,-3.719286,-2.376736 \backslash C, 0,-2.042375,-4.16268,0.130858 \backslash C, 0,-2.31$ $1137,-5.588349,0.510714 \mathrm{H}, 0,-1.433386,-6.052906,0.984684 \mathrm{H}, 0,-2.602451$ ,-6.16798,-0.378219\H,0,-3.149429,-5.631146,1.230331\N,0,-1.091651,-3. $466703,0.713571 \mathrm{H}, 0,-0.949884,-2.413026,0.497999 \mathrm{CC}, 0,-0.178668,-3.8934$ $36,1.730052 \mathrm{IC}, 0,-0.626253,-4.461955,2.93571 \backslash \mathrm{H}, 0,-1.695661,-4.610315,3$. $108838 \mathrm{IC}, 0,0.310682,-4.802388,3.921425 \mathrm{VH}, 0,-0.034894,-5.241385,4.86323$ $8|\mathrm{C}, 0,1.678544,-4.569771,3.709209| \mathrm{C}, 0,2.112935,-3.981807,2.509849 \backslash \mathrm{H}, 0$, $3.1761,-3.7859,2.342826 \backslash \mathrm{C}, 0,1.18772,-3.629951,1.519087 \backslash \mathrm{H}, 0,1.514046,-3$ $.161043,0.585996 \mathrm{IH}, 0,2.405947,-4.836384,4.483184 \mathrm{IC}, 0,-5.208387,-1.6695$ $,-4.07293 \backslash \mathrm{H}, 0,-6.227688,-2.084671,-4.127276 \backslash \mathrm{H}, 0,-5.280418,-0.580559,-3$ $.89948 \mathrm{H}, 0,-4.724286,-1.803584,-5.058165 \mathrm{IV}$ Version=ES64L-G09RevD.01।Sta te=1-AlHF=-3216.0905493IRMSD=5.168e-09IDipole $=-2.7069863,-4.2381048,0$. 9582992lQuadrupole $=0.0261353,18.6984818,-18.7246171,21.4205092,9.55934$ 68,-10.2331119|PG=C01 [X(C65H72N1O4P1)]॥@

\section{TRIP/1E Switching TS}

Confo

1\1|GINC-WORKER2ISPIRTPSSTPSSIdef2SVPIC65H72N1O4P1\JHIOEI26-Mar-201910

II\#p tpsstpss/def2svp int=ultrafine empiricaldispersion=gd3 scrf=(smd, solvent=ch2cl2,read,externaliteration, dovacuum) Ititlel|0,1|P,0,0.0347 $82,-0.165131,-0.152115 \backslash 0,0,1.395118,0.615765,0.456654 \backslash 0,0,-0.826611,1$ $19423,-0.66295310,0,-0.625713,-0.817496,1.04082910,0,0.317299,-0.97324$ $3,-1.399012|C, 0,1.351228,2.899856,-0.272484| C, 0,1.98252,1.65199,-0.235$ $017 \backslash \mathrm{C}, 0,3.271277,1.411134,-0.807025 \backslash \mathrm{C}, 0,3.900111,2.460424,-1.462631 \backslash \mathrm{H}$, $0,4.897848,2.30996,-1.888631 \backslash \mathrm{C}, 0,3.267375,3.727401,-1.623785 \backslash \mathrm{C}, 0,1.966$ $006,3.952863,-1.041553 \backslash \mathrm{C}, 0,1.327911,5.207524,-1.281533 \backslash \mathrm{H}, 0,0.323092,5$ $377904,-0.884041 \backslash \mathrm{C}, 0,1.95485,6.200821,-2.019577 \backslash \mathrm{H}, 0,1.440723,7.152252$, $-2.195457 \backslash \mathrm{C}, 0,3.253765,5.991777,-2.555978 \mathrm{H}, 0,3.741232,6.786757,-3.130$ 902।C, $0,3.891614,4.776185,-2.364417 \backslash \mathrm{H}, 0,4.884909,4.594878,-2.791038 \mathrm{IC}$, $0,0.077948,3.091445,0.478858 \backslash \mathrm{C}, 0,-0.988481,2.214664,0.25447 \backslash \mathrm{C}, 0,-2.264$ $24,2.35618,0.888059 \mathrm{IC}, 0,-2.43437,3.41356,1.771162 \mathrm{H}, 0,-3.410905,3.5740$ $55,2.239146 \mathrm{IC}, 0,-1.364094,4.29248,2.107361 \backslash \mathrm{C}, 0,-0.078521,4.122575,1.47$ $5144 \backslash \mathrm{C}, 0,0.995639,4.964708,1.895611 \mathrm{H}, 0,1.988817,4.819931,1.461124 \backslash \mathrm{C}, 0$ $, 0.801391,5.949359,2.853338 \mathrm{H}, 0,1.643463,6.578019,3.163334 \backslash \mathrm{C}, 0,-0.4771$ $45,6.14448,3.441148 \mathrm{H}, 0,-0.618867,6.930265,4.191178 \mathrm{IC}, 0,-1.535202,5.32$ $691,3.076404 \mathrm{IH}, 0,-2.520897,5.451176,3.539352 \mathrm{CC}, 0,-3.380599,1.409513,0$. $556978 \backslash \mathrm{C}, 0,-3.766417,0.39074,1.473954 \backslash \mathrm{C}, 0,-4.060068,1.541095,-0.681035$ IC $, 0,-4.831272,-0.456868,1.126023|\mathrm{C}, 0,-5.116217,0.657797,-0.986085| C, 0$ $,-5.522174,-0.349607,-0.096511 \backslash \mathrm{H}, 0,-5.140171,-1.234339,1.834327 \backslash \mathrm{H}, 0,-5$ $.62806,0.773603,-1.946566 \backslash C, 0,3.899003,0.058863,-0.662256 \backslash C, 0,4.021328$ $,-0.801348,-1.788542 \backslash C, 0,4.356021,-0.370854,0.612701 \backslash C, 0,4.60803,-2.06$ $8321,-1.614431 \backslash \mathrm{C}, 0,4.942555,-1.644713,0.731872 \backslash \mathrm{C}, 0,5.075215,-2.512328$, $-0.364831 \backslash \mathrm{H}, 0,4.701351,-2.729243,-2.484023 \backslash \mathrm{H}, 0,5.307356,-1.974821,1.71$ 234IC, $0,4.252301,0.517971,1.85379 \backslash \mathrm{H}, 0,3.74548,1.452205,1.559324 \backslash \mathrm{C}, 0,3$. $544717,-0.389383,-3.183504 \backslash \mathrm{C}, 0,5.702382,-3.89393,-0.198682 \backslash \mathrm{H}, 0,5.97310$ $2,-4.000099,0.869016 \mid \mathrm{C}, 0,4.745837,-0.116287,-4.112977 \backslash \mathrm{H}, 0,5.415963,0.6$ $54386,-3.691807 \backslash \mathrm{H}, 0,5.343173,-1.034112,-4.267986 \mathrm{IH}, 0,4.40043,0.234442$, $-5.103072 \mathrm{IC}, 0,6.996515,-4.031905,-1.026487 \backslash \mathrm{H}, 0,7.724794,-3.243881,-0.7$ $63606 \backslash \mathrm{H}, 0,7.472893,-5.013473,-0.847739 \mathrm{H}, 0,6.786657,-3.952557,-2.10918$ $6 \mathrm{IC}, 0,4.70201,-5.016596,-0.543161 \backslash \mathrm{H}, 0,4.404266,-4.972532,-1.607003 \backslash \mathrm{H}, 0$ $, 5.151955,-6.009949,-0.361031 \backslash \mathrm{H}, 0,3.785583,-4.937176,0.067758 \mathrm{lC}, 0,3.39$ $9063,-0.136749,2.959014 \mathrm{H}, 0,2.398116,-0.396808,2.577222 \mathrm{H}, 0,3.877927,-$ $1.054359,3.34563 \backslash \mathrm{H}, 0,3.274246,0.558682,3.809561 \backslash \mathrm{C}, 0,5.649321,0.909516$, $2.378967 \backslash \mathrm{H}, 0,5.560817,1.601657,3.236598 \mathrm{H}, 0,6.215387,0.022441,2.718622$ $\mathrm{IH}, 0,6.243485,1.41232,1.594725 \mathrm{IC}, 0,-6.657383,-1.332812,-0.396569 \mathrm{|H}, 0,-$ $7.316098,-1.327375,0.494822 \backslash \mathrm{C}, 0,-3.074852,0.218841,2.828633 \backslash \mathrm{H}, 0,-2.080$ $942,0.689451,2.742991 \backslash \mathrm{C}, 0,-3.68988,2.637435,-1.682661 \backslash \mathrm{H}, 0,-2.858451,3$. $218999,-1.249717 \backslash \mathrm{C}, 0,-7.51647,-0.947716,-1.610518 \backslash \mathrm{H}, 0,-6.935192,-0.992$ $848,-2.549846 \mathrm{H}, 0,-8.364331,-1.648107,-1.713818 \mathrm{H}, 0,-7.929611,0.072226$ $,-1.513456 \mathrm{IC}, 0,-6.119704,-2.774612,-0.547685 \mathrm{IH}, 0,-5.534566,-3.081992,0$ $.336493 \backslash \mathrm{H}, 0,-6.955354,-3.488622,-0.666636 \backslash \mathrm{H}, 0,-5.467257,-2.859491,-1.4$ $34175 \backslash \mathrm{C}, 0,-3.189011,2.056534,-3.019389 \mathrm{IH}, 0,-2.843159,2.868476,-3.68534$ $1 \mathrm{H}, 0,-2.34572,1.36506,-2.854252 \mathrm{H}, 0,-3.994048,1.51418,-3.548327 \backslash \mathrm{C}, 0,-$ $4.86001,3.618404,-1.900332 \backslash \mathrm{H}, 0,-5.186075,4.06813,-0.945194 \backslash \mathrm{H}, 0,-4.5578$ $04,4.437516,-2.578665 \backslash \mathrm{H}, 0,-5.73219,3.111763,-2.353347 \backslash \mathrm{C}, 0,-3.863027,0$. $936457,3.946023 \mathrm{lH}, 0,-4.8677,0.488842,4.064869 \backslash \mathrm{H}, 0,-3.334414,0.847131,4$ $.91321 \mathrm{H}, 0,-3.997567,2.00997,3.730229 \mathrm{IC}, 0,-2.842238,-1.257976,3.201445$ $\mathrm{IH}, 0,-2.220855,-1.323161,4.113605 \backslash \mathrm{H}, 0,-3.789612,-1.787703,3.413655 \backslash \mathrm{H}, 0$ 
$-2.307888,-1.777944,2.39209|\mathrm{C}, 0,2.580322,-1.420625,-3.802364| \mathrm{H}, 0,2.22$ $5823,-1.06323,-4.787197 \backslash \mathrm{H}, 0,3.071073,-2.398414,-3.9632391 \mathrm{H}, 0,1.705131$, $-1.560087,-3.146214 \mid \mathrm{H}, 0,2.981548,0.553464,-3.079022 \mathrm{IC}, 0,1.695809,-3.94$ $1035,3.341706|\mathrm{C}, 0,0.443036,-4.459186,3.7391| \mathrm{H}, 0,0.310871,-4.824163,4.7$ $63591 \backslash \mathrm{C}, 0,-0.632039,-4.511891,2.850791 \backslash \mathrm{H}, 0,-1.59072,-4.906073,3.198842$ IC, $0,-0.496914,-4.023504,1.527638 \mid \mathrm{C}, 0,0.761401,-3.505219,1.125875 \mathrm{IH}, 0$, $0.914965,-3.148088,0.1024 \mathrm{IC}, 0,1.83326,-3.476085,2.015819 \mathrm{lH}, 0,2.794403$, $-3.078829,1.676271|\mathrm{C}, 0,-1.61377,-4.059379,0.582176| \mathrm{C}, 0,-2.721921,-5.05$ $2504,0.728196 \mathrm{H}, 0,-3.023038,-5.101359,1.787457 \mathrm{H}, 0,-2.373688,-6.062608$ $, 0.4468111 \mathrm{H}, 0,-3.59717,-4.787234,0.118716 \mathrm{IN}, 0,-1.604478,-3.176801,-0.4$ $02341 \mid \mathrm{H}, 0,-0.943675,-2.378492,-0.295389 \mathrm{CC}, 0,-2.405532,-3.118456,-1.578$ $136 \mathrm{IC}, 0,-2.714378,-4.282657,-2.308361 \backslash \mathrm{H}, 0,-2.345675,-5.25665,-1.976569$ IC, $0,-3.462007,-4.1758,-3.487633 \mid \mathrm{H}, 0,-3.698474,-5.080465,-4.057323 \mathrm{IC}, 0$ $,-3.885232,-2.918264,-3.946674|\mathrm{C}, 0,-3.556632,-1.762349,-3.22084| \mathrm{H}, 0,-3$ $.886226,-0.780616,-3.570546 \mid \mathrm{C}, 0,-2.818399,-1.852728,-2.0354511 \mathrm{H}, 0,-2.5$ $84749,-0.954714,-1.458357 \mathrm{IH}, 0,-4.464254,-2.838021,-4.872534 \mathrm{IC}, 0,2.8455$ $43,-3.856436,4.31255 \backslash \mathrm{H}, 0,2.80809,-4.668387,5.058555 \backslash \mathrm{H}, 0,3.816575,-3.89$ $1144,3.791147 \mathrm{IH}, 0,2.800106,-2.898729,4.866083 \|$ Version=ES64L-G09RevD.0 1|State=1-AlHF=-3216.0860407/RMSD=4.893e-09|Dipole=-1.7733435,-4.49895 $5,0.994833 \mid$ Quadrupole $=-9.1398178,22.5881989,-13.4483811,16.5807397,9.9$ 252408,-8.5907668/PG=C01 [X(C65H72N1O4P1)]।@

Conf1

1/1IGINC-WORKER1ISPIRTPSSTPSSIdef2SVPIC65H72N1O4P1/JHIOE|29-Mar-201910

॥\#p tpsstpss/def2svp int=ultrafine empiricaldispersion=gd3 scrf=(smd, solvent $=\mathrm{ch} 2 \mathrm{cl} 2$, read, externaliteration, dovacuum) Ititle $\mid \mathbf{0}, 1 \backslash \mathrm{P}, 0,0.0317$ $69,-0.176859,-0.219803 \backslash 0,0,1.418689,0.536058,0.414616 \backslash 0,0,-0.77859,1.2$ $27426,-0.690323 \backslash 0,0,-0.657435,-0.838211,0.951711 \backslash 0,0,0.289369,-0.95859$ $9,-1.488712 \backslash \mathrm{C}, 0,1.427718,2.8595,-0.184595 \backslash \mathrm{C}, 0,2.027652,1.595618,-0.220$ $777 \backslash \mathrm{C}, 0,3.305467,1.355583,-0.817287 \backslash \mathrm{C}, 0,3.958949,2.425167,-1.413022 \backslash \mathrm{H}$ $0,4.949277,2.273806,-1.855198 \backslash \mathrm{C}, 0,3.36249,3.716958,-1.490768 \mathrm{IC}, 0,2.069$ $433,3.941388,-0.890402 \mathrm{IC}, 0,1.466791,5.226079,-1.051805 \mathrm{IH}, 0,0.467383,5$. $400853,-0.643326 \mathrm{IC}, 0,2.120949,6.245672,-1.727772 \mathrm{H}, 0,1.633384,7.220135$ ,-1.842413।C,0,3.412696,6.034509,-2.280165\H,0,3.921687,6.849789,-2.80 $5861 \backslash \mathrm{C}, 0,4.01511,4.791424,-2.167589 \mathrm{IH}, 0,5.001546,4.607846,-2.608852 \mathrm{CC}$, $0,0.151002,3.039016,0.564578 \backslash \mathrm{C}, 0,-0.933646,2.20553,0.273144 \backslash \mathrm{C}, 0,-2.219$ $242,2.350659,0.885529 \backslash \mathrm{C}, 0,-2.382197,3.368657,1.81478 \backslash \mathrm{H}, 0,-3.36535,3.53$ $3898,2.267031 \backslash \mathrm{C}, 0,-1.297018,4.201135,2.215762 \backslash \mathrm{C}, 0,-0.001497,4.024943,1$ $.606142 \backslash \mathrm{C}, 0,1.083054,4.819839,2.08732 \backslash \mathrm{H}, 0,2.082047,4.667534,1.668912 \backslash \mathrm{C}$ $, 0,0.890747,5.767804,3.081726 \backslash \mathrm{H}, 0,1.740392,6.361331,3.437034 \backslash \mathrm{C}, 0,-0.39$ $6159,5.970708,3.648342 \backslash \mathrm{H}, 0,-0.53602,6.727629,4.42783 \backslash \mathrm{C}, 0,-1.464912,5.1$ $95814,3.226108 \mathrm{H}, 0,-2.457912,5.325233,3.671714 \backslash \mathrm{C}, 0,-3.352219,1.453263$ $0.482006 \backslash \mathrm{C}, 0,-3.792002,0.405462,1.339442 \backslash \mathrm{C}, 0,-3.9883,1.660205,-0.77054$ $8|\mathrm{C}, 0,-4.860597,-0.406805,0.918768| \mathrm{C}, 0,-5.058308,0.823355,-1.140625 \mathrm{IC}$, $0,-5.507103,-0.22057,-0.316219 \backslash \mathrm{H}, 0,-5.197592,-1.214768,1.577085 \mathrm{H}, 0,-5$ $.551423,0.983291,-2.107331 \backslash \mathrm{C}, 0,3.903399,-0.017518,-0.766061 \backslash \mathrm{C}, 0,3.9709$ $47,-0.812691,-1.941039 \backslash \mathrm{C}, 0,4.40067,-0.524261,0.466075 \backslash \mathrm{C}, 0,4.560196,-2$. $089954,-1.861773|C, 0,4.975281,-1.807303,0.493793| C, 0,5.070629,-2.60722$ $1,-0.660003 \mathrm{H}, 0,4.623361,-2.705063,-2.767682 \backslash \mathrm{H}, 0,5.365395,-2.190344,1$. $444285 \backslash \mathrm{C}, 0,4.357283,0.301453,1.753497 \backslash \mathrm{H}, 0,3.848425,1.253785,1.528653 \mathrm{IC}$ $, 0,3.440335,-0.312001,-3.286507 \backslash \mathrm{C}, 0,5.712154,-3.991781,-0.614583 \backslash \mathrm{H}, 0,5$ $.680149,-4.396117,-1.64385 \backslash \mathrm{C}, 0,4.603326,0.083266,-4.220875 \mathrm{IH}, 0,5.25587$ $3,0.843292,-3.755822 \mathrm{H}, 0,5.229848,-0.795026,-4.464616 \mathrm{H}, 0,4.216434,0.4$ $98584,-5.169825 \backslash \mathrm{C}, 0,4.922722,-4.959261,0.290654 \mathrm{IH}, 0,3.869614,-5.038007$ $,-0.031395 \mathrm{|H}, 0,5.367368,-5.97097,0.260701 \backslash \mathrm{H}, 0,4.932929,-4.619604,1.342$ $57 \backslash \mathrm{C}, 0,7.192418,-3.916265,-0.187495 \mathrm{H}, 0,7.291203,-3.537955,0.846804 \backslash \mathrm{H}$, $0,7.661464,-4.916648,-0.225796 \mathrm{H}, 0,7.765636,-3.243685,-0.850572 \mathrm{IC}, 0,3$. $544401,-0.400309,2.859068 \mathrm{H}, 0,2.519853,-0.616916,2.514056 \mathrm{H}, 0,4.019624$ ,-1.349191,3.165135\H,0,3.477686,0.243446,3.755562।C,0,5.780356,0.6526 $68,2.235756 \backslash \mathrm{H}, 0,5.738351,1.300315,3.130904 \backslash \mathrm{H}, 0,6.350539,-0.256314,2.50$ $3376 \mathrm{lH}, 0,6.344955,1.188862,1.45175 \backslash \mathrm{C}, 0,-6.670022,-1.112285,-0.74439 \mathrm{IH}$, $0,-6.890206,-0.869458,-1.801149 \backslash \mathrm{C}, 0,-3.152729,0.159865,2.708082 \backslash \mathrm{H}, 0,-2$ $.149681,0.618015,2.682671 \backslash \mathrm{C}, 0,-3.548256,2.776538,-1.719625 \backslash \mathrm{H}, 0,-2.7021$ $25,3.303745,-1.247493 \mathrm{IC}, 0,-6.318569,-2.612033,-0.673633 \mathrm{H}, 0,-6.129279$, $-2.933688,0.366825 \mathrm{H}, 0,-7.152928,-3.222678,-1.064329 \backslash \mathrm{H}, 0,-5.418552,-2$. $838767,-1.270658 \mathrm{IC}, 0,-7.935839,-0.801937,0.081586 \mathrm{IH}, 0,-8.219776,0.2618$ $83,-0.007334 \mathrm{H}, 0,-8.789167,-1.41559,-0.261722 \mathrm{H}, 0,-7.77002,-1.020259,1$ $.15294 \backslash \mathrm{C}, 0,-3.043701,2.219374,-3.0655 \backslash \mathrm{H}, 0,-2.652124,3.037291,-3.698015$ $\mathrm{IH}, 0,-2.231928,1.48962,-2.906314 \mathrm{H}, 0,-3.857571,1.72504,-3.627002 \backslash \mathrm{C}, 0,-$ $4.668983,3.815858,-1.923816 \mathrm{H}, 0,-4.995392,4.243633,-0.95876 \mathrm{H}, 0,-4.315$ $855,4.645185,-2.564017 \backslash \mathrm{H}, 0,-5.552674,3.365957,-2.413032 \backslash \mathrm{C}, 0,-3.974209$, $0.837952,3.826372 \backslash \mathrm{H}, 0,-4.988564,0.400581,3.885857 \backslash \mathrm{H}, 0,-3.484975,0.6975$ $31,4.808128 \mathrm{H}, 0,-4.086523,1.921968,3.654658 \backslash \mathrm{C}, 0,-2.954855,-1.335526,3$. $021406 \backslash \mathrm{H}, 0,-2.374806,-1.450346,3.955533 \backslash \mathrm{H}, 0,-3.916381,-1.862265,3.1665$ $36 \mathrm{H}, 0,-2.390611,-1.823382,2.212641 \mathrm{CC}, 0,2.49795,-1.32398,-3.967179 \mathrm{lH}, 0$ $, 2.096281,-0.893698,-4.903479 \backslash \mathrm{H}, 0,3.020903,-2.260833,-4.234265 \backslash \mathrm{H}, 0,1.6$ $50249,-1.558023,-3.302213 \backslash \mathrm{H}, 0,2.845796,0.596007,-3.087808 \backslash \mathrm{C}, 0,1.603097$ 
,-4.024179,3.193087\C,0,0.342626,-4.525481,3.587529)H, 0,0.207476,-4.90 $1958,4.607467 \backslash \mathrm{C}, 0,-0.736852,-4.544562,2.703305 \mathrm{IH}, 0,-1.701875,-4.922286$ ,3.051638\C,0,-0.59754,-4.041606,1.386019\C,0,0.669637,-3.543003,0.986 $744 \mathrm{IH}, 0,0.826488,-3.175166,-0.032372 \mathrm{IC}, 0,1.744486,-3.543804,1.873064 \mathrm{IH}$ $, 0,2.711314,-3.160924,1.534453 \backslash \mathrm{C}, 0,-1.721413,-4.034314,0.448466 \backslash \mathrm{C}, 0,-2$ $860233,-4.9931,0.591063 \mathrm{H}, 0,-3.139041,-5.068198,1.654539 \mathrm{H}, 0,-2.55443$ $3,-6.004348,0.267203 \mathrm{H}, 0,-3.739239,-4.676209,0.011601 \backslash \mathrm{N}, 0,-1.692569,-3$ $.14139,-0.526797 \backslash \mathrm{H}, 0,-1.008789,-2.363599,-0.414938 \mathrm{IC}, 0,-2.505693,-3.04$ $1688,-1.691237 \backslash \mathrm{C}, 0,-2.863484,-4.184849,-2.43272 \backslash \mathrm{H}, 0,-2.521768,-5.17525$ $7,-2.121227 \backslash \mathrm{C}, 0,-3.62703,-4.036466,-3.597119 \mathrm{H}, 0,-3.902376,-4.924547,-$ $4.175401 \backslash \mathrm{C}, 0,-4.01705,-2.759369,-4.03129 \backslash \mathrm{C}, 0,-3.63766,-1.62511,-3.2964$ $49 \mathrm{H}, 0,-3.937307,-0.627243,-3.62775 \backslash \mathrm{C}, 0,-2.882816,-1.756737,-2.125471 \backslash$ $\mathrm{H}, 0,-2.609138,-0.874688,-1.541526 \backslash \mathrm{H}, 0,-4.60845,-2.647448,-4.946003 \backslash \mathrm{C}, 0$

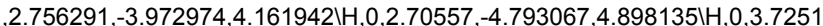
$82,-4.018971,3.637602 \backslash \mathrm{H}, 0,2.7306,-3.021276,4.726917 \mathrm{IV}$ Version=ES64L-G09 RevD.01IState=1-AlHF=-3216.0871784|RMSD=4.771e-09|Dipole=-1.8566272,-4 $.4193497,0.9127594 \mid Q u a d r u p o l e=-7.758896,21.7723652,-14.0134691,16.7206$ 18,11.2113056,-7.0648878IPG=C01 [X(C65H72N1O4P1)]॥@

Conf2

1।1\GINC-WORKER2ISPIRTPSSTPSSIdef2SVPIC65H72N1O4P1\JHIOEI01-Apr-201910

II\#p tpsstpss/def2svp int=ultrafine empiricaldispersion=gd3 scrf=(smd, solvent $=$ ch2cl2, read, externaliteration, dovacuum) |ltitlell0,1।P, $0,0.0593$ $84,-0.178424,-0.168572 \backslash 0,0,1.407615,0.596532,0.472944 \backslash 0,0,-0.788341,1$. $18385,-0.696524 \backslash 0,0,-0.629622,-0.83046,1.008195 \backslash 0,0,0.367884,-0.985082$ $,-1.410367 \backslash \mathrm{C}, 0,1.36528,2.895659,-0.207176 \backslash \mathrm{C}, 0,2.002058,1.650386,-0.184$ $761 \backslash \mathrm{C}, 0,3.303145,1.428222,-0.735448 \mathrm{IC}, 0,3.942329,2.495551,-1.350364 \mathrm{IH}$, $0,4.949772,2.35926,-1.757751 \backslash \mathrm{C}, 0,3.309841,3.765133,-1.490316 \mathrm{IC}, 0,1.994$ $381,3.971138,-0.933112 \mathrm{lC}, 0,1.358419,5.230356,-1.154738 \mathrm{H}, 0,0.343398,5$. $387445,-0.778416 \mathrm{IC}, 0,2.000355,6.245544,-1.848863 \mathrm{H}, 0,1.487611,7.20018$ $-2.010856 \backslash \mathrm{C}, 0,3.312937,6.05511,-2.358089 \backslash \mathrm{H}, 0,3.812246,6.867269,-2.8977$ 22IC $, 0,3.949151,4.835888,-2.185412 \mathrm{lH}, 0,4.953183,4.668718,-2.592179 \mathrm{IC}, 0$ $0.069431,3.062647,0.51113 \backslash \mathrm{C}, 0,-0.987229,2.19082,0.22866 \backslash \mathrm{C}, 0,-2.288147$ ,2.325085,0.810449IC,0,-2.491111,3.361772,1.710771) $, 0,-3.486355,3.516$ $134,2.139844 \backslash C, 0,-1.432644,4.228362,2.110727 \backslash \mathrm{C}, 0,-0.124308,4.071169,1$. $523827 \backslash \mathrm{C}, 0,0.933326,4.903132,2.002336 \mathrm{IH}, 0,1.94193,4.766814,1.601867 \backslash \mathrm{C}$ $0,0.703664,5.865847,2.974435 \mathrm{lH}, 0,1.533477,6.486941,3.329554 \mathrm{IC}, 0,-0.595$ $851,6.048306,3.518809 \backslash \mathrm{H}, 0,-0.765219,6.816877,4.280884 \backslash \mathrm{C}, 0,-1.639482,5$ $239594,3.097112 \backslash \mathrm{H}, 0,-2.641877,5.353921,3.525452 \backslash \mathrm{C}, 0,-3.396826,1.399765$ $, 0.40194 \backslash \mathrm{C}, 0,-3.829426,0.353548,1.264598 \mid \mathrm{C}, 0,-4.019825,1.581873,-0.861$ $244 \backslash \mathrm{C}, 0,-4.876005,-0.483974,0.838089 \backslash \mathrm{C}, 0,-5.06844,0.720453,-1.236725 \backslash \mathrm{C}$ $, 0,-5.508951,-0.323054,-0.407355 \mathrm{lH}, 0,-5.206752,-1.291104,1.500541 \mathrm{H}, 0$ $-5.551177,0.860569,-2.21171 \backslash \mathrm{C}, 0,3.934199,0.075608,-0.609036 \backslash \mathrm{C}, 0,4.0667$ $37,-0.765296,-1.748403 \backslash \mathrm{C}, 0,4.385708,-0.371796,0.66125 \backslash C, 0,4.661358,-2$ $030851,-1.592076 \backslash C, 0,4.980983,-1.643248,0.762932 \backslash C, 0,5.12595,-2.491444$ $-0.347255 \mathrm{H}, 0,4.76414,-2.677203,-2.471466 \mathrm{H}, 0,5.342316,-1.987001,1.74$ $0004 \mathrm{IC}, 0,4.261952,0.493988,1.916572 \mathrm{H}, 0,3.748037,1.428137,1.634172 \mathrm{IC}, 0$ ,3.596695,-0.327772,-3.137765।C $0,5.761417,-3.871489,-0.200166 \mathrm{\backslash H}, 0,6.0$ $33239,-3.990468,0.865893 \mathrm{CC}, 0,4.802876,0.005496,-4.040745 \backslash \mathrm{H}, 0,5.444785$ $0.781757,-3.587479 \mathrm{H}, 0,5.427714,-0.890785,-4.212417 \mathrm{VH}, 0,4.462042,0.375$ $007,-5.025603 \backslash \mathrm{C}, 0,7.05599,-3.991815,-1.0301 \backslash \mathrm{H}, 0,7.780342,-3.203439,-0$ $75757 \backslash \mathrm{H}, 0,7.537602,-4.973059,-0.863957 \backslash \mathrm{H}, 0,6.845478,-3.899986,-2.11168$ $4 \mathrm{IC}, 0,4.767129,-4.995059,-0.559432 \mathrm{WH}, 0,4.468007,-4.937623,-1.622268 \mathrm{WH}$ $0,5.223055,-5.988251,-0.391784 \backslash \mathrm{H}, 0,3.850796,-4.929814,0.053311 \backslash \mathrm{C}, 0,3.4$ $044,-0.189392,3.000841 \backslash \mathrm{H}, 0,2.409283,-0.451294,2.604905 \backslash \mathrm{H}, 0,3.887831,-1$ $.110053,3.374104 \backslash \mathrm{H}, 0,3.265343,0.488442,3.863327 \backslash \mathrm{C}, 0,5.649569,0.890067$ $2.462295 \mathrm{IH}, 0,5.545615,1.561626,3.33439 \mathrm{IH}, 0,6.223325,0.002293,2.786925 \backslash$ $\mathrm{H}, 0,6.243891,1.41705,1.694209 \mathrm{IC}, 0,-6.65117,-1.239166,-0.840045 \mathrm{IH}, 0,-6$. $861622,-1.012791,-1.902414 \backslash \mathrm{C}, 0,-3.206157,0.137465,2.645603 \backslash \mathrm{H}, 0,-2.2080$ $25,0.606583,2.626411 \backslash \mathrm{C}, 0,-3.585304,2.693697,-1.818403 \backslash \mathrm{H}, 0,-2.768753,3$ $254276,-1.332685 \backslash \mathrm{C}, 0,-6.278446,-2.732477,-0.745946 \backslash \mathrm{H}, 0,-6.092528,-3.03$ $7084,0.30025 \mathrm{H}, 0,-7.101377,-3.360074,-1.1341111 \mathrm{H}, 0,-5.371325,-2.955001$ $-1.333782 \backslash \mathrm{C}, 0,-7.932266,-0.937792,-0.034201 \backslash \mathrm{H}, 0,-8.229677,0.120913,-0$ $.138796 \mathrm{H}, 0,-8.772387,-1.56716,-0.381588 \backslash \mathrm{H}, 0,-7.777501,-1.141939,1.041$ $602 \backslash \mathrm{C}, 0,-3.023956,2.124517,-3.136717 \backslash \mathrm{H}, 0,-2.634621,2.940279,-3.773441 \backslash$ $\mathrm{H}, 0,-2.198417,1.420538,-2.937567 \backslash \mathrm{H}, 0,-3.806419,1.597134,-3.712728 \mathrm{lC}, 0$, $-4.72824,3.695899,-2.07815 \backslash \mathrm{H}, 0,-5.094933,4.135389,-1.133053 \backslash \mathrm{H}, 0,-4.379$ $212,4.52055,-2.726533 \mathrm{H}, 0,-5.584175,3.211168,-2.582987 \backslash \mathrm{C}, 0,-4.050993,0$ $.823358,3.741402 \backslash \mathrm{H}, 0,-5.06143,0.376043,3.792683 \backslash \mathrm{H}, 0,-3.57482,0.702645$ $4.732156 \mathrm{VH}, 0,-4.171827,1.903442,3.551841 \mathrm{lC}, 0,-2.995601,-1.350342,2.985$ $141 \mathrm{H}, 0,-2.422582,-1.444632,3.925803 \backslash \mathrm{H}, 0,-3.953027,-1.884568,3.12973 \backslash \mathrm{H}$ $, 0,-2.419133,-1.845171,2.18948 \mid \mathrm{C}, 0,2.669707,-1.364942,-3.801894 \backslash \mathrm{H}, 0,2$ $316598,-0.985053,-4.778719 \mathrm{H}, 0,3.189399,-2.322775,-3.989439 \backslash \mathrm{H}, 0,1.7909$ $1,-1.549867,-3.162014 \backslash \mathrm{H}, 0,3.006888,0.596385,-3.013297 \backslash \mathrm{C}, 0,1.714114,-3$ $994284,3.29239 \backslash \mathrm{C}, 0,0.459589,-4.508249,3.689444 \backslash \mathrm{H}, 0,0.329566,-4.883895$, $4.710342 \mathrm{IC}, 0,-0.619923,-4.543322,2.805653 \backslash \mathrm{H}, 0,-1.579695,-4.933607,3.15$ $477 \backslash \mathrm{C}, 0,-0.487006,-4.043876,1.486402 \backslash \mathrm{C}, 0,0.773095,-3.528768,1.085396 \mathrm{IH}$ 
,0,0.925784,-3.161369,0.065405\C,0,1.848407,-3.514136, 1.971468\H,0,2.8 $10279,-3.118317,1.632115 \backslash \mathrm{C}, 0,-1.608116,-4.063051,0.545368 \backslash \mathrm{C}, 0,-2.73470$ $5,-5.034879,0.695665 \backslash \mathrm{H}, 0,-3.018199,-5.09724,1.758772 \backslash \mathrm{H}, 0,-2.414735,-6$. $046964,0.38865 \mathrm{IH}, 0,-3.614794,-4.738029,0.107184 \mathrm{IN}, 0,-1.588418,-3.18096$ $7,-0.439579 \mathrm{IH}, 0,-0.916432,-2.392153,-0.334638 \mathrm{IC}, 0,-2.39385,-3.108225,-$ $1.611039 \mid \mathrm{C}, 0,-2.725231,-4.266461,-2.341309 \mathrm{IH}, 0,-2.373131,-5.247102,-2$ $011142 \mathrm{IC}, 0,-3.473659,-4.145245,-3.518506 \mathrm{lH}, 0,-3.727896,-5.045003,-4.08$ $8321 \backslash \mathrm{C}, 0,-3.874539,-2.879726,-3.976092 \backslash \mathrm{C}, 0,-3.523811,-1.730454,-3.2504$ $31 \mathrm{H}, 0,-3.833818,-0.741908,-3.599123 \backslash \mathrm{C}, 0,-2.785743,-1.834918,-2.066036$ $\mathrm{IH}, 0,-2.534929,-0.941253,-1.48914 \backslash \mathrm{H}, 0,-4.452985,-2.788534,-4.901331 \backslash \mathrm{C}$, $0,2.868934,-3.931179,4.258693 \backslash \mathrm{H}, 0,2.834331,-4.758753,4.987529 \backslash \mathrm{H}, 0,3.83$ $7119,-3.955825,3.731606 \mathrm{H}, 0,2.827342,-2.985866,4.833379|| V e r s i o n=E S 64 \mathrm{~L}$ -G09RevD.01।State=1-AlHF=-3216.0870195\RMSD=4.730e-09\Dipole $=-1.791023$ $9,-4.4433182,0.95199081$ Quadrupole $=-9.1511528,22.5402497,-13.3890968,16$ .0129477,11.0477293,-8.3206864\PG=C01 [X(C65H72N1O4P1)]॥@

Conf3

1\1\GINC-WORKER0ISPIRTPSSTPSSIdef2SVPIC65H72N1O4P1\JHIOEI29-Apr-201910

II\#p tpsstpss/def2svp int=ultrafine empiricaldispersion=gd3 scrf=(smd, solvent $=$ ch2cl2,read,externaliteration, dovacuum) $\mid$ title $\| 0,1 \backslash \mathrm{P}, 0,0.0226$ $98,-0.175332,-0.184181 \backslash 0,0,1.414735,0.560129,0.410699 \backslash 0,0,-0.820413,1$. $21519,-0.63810,0,-0.624708,-0.844138,1.006991 \backslash 0,0,0.257783,-0.957818,-$ $1.456772 \backslash \mathrm{C}, 0,1.385562,2.87523,-0.217747 \backslash \mathrm{C}, 0,1.997437,1.617185,-0.25297$ $1|\mathrm{C}, 0,3.262497,1.382103,-0.878053| \mathrm{C}, 0,3.89088,2.451192,-1.501437 \backslash \mathrm{H}, 0,4$ $.871139,2.303636,-1.966944 \mid C, 0,3.279304,3.735881,-1.580342 \backslash C, 0,1.99811$ $9,3.954072,-0.952957 \backslash \mathrm{C}, 0,1.376641,5.229402,-1.116833 \backslash \mathrm{H}, 0,0.384747,5.39$ $7377,-0.687431 \backslash \mathrm{C}, 0,2.003477,6.247501,-1.820311 \mathrm{~W}, 0,1.502302,7.214859,-$ $1.936564 \backslash \mathrm{C}, 0,3.284929,6.043727,-2.398994 \mathrm{H}, 0,3.772701,6.857941,-2.9460$ $39 \mathrm{IC}, 0,3.904195,4.809053,-2.284793 \mathrm{IH}, 0,4.882379,4.631171,-2.746342 \mathrm{IC}, 0$ $, 0.128831,3.052571,0.564631 \backslash C, 0,-0.954966,2.203243,0.318247 \backslash C, 0,-2.222$ $348,2.34322,0.968568 \backslash \mathrm{C}, 0,-2.368385,3.37376,1.886933 \backslash \mathrm{H}, 0,-3.338664,3.53$ $4755,2.367619 \backslash \mathrm{C}, 0,-1.280478,4.222456,2.243708 \backslash \mathrm{C}, 0,-0.002078,4.050364,1$ $.597916 \mathrm{IC}, 0,1.089007,4.860439,2.037437 \backslash \mathrm{H}, 0,2.076709,4.711781,1.591893 \backslash$ $\mathrm{C}, 0,0.917359,5.818902,3.025522 \backslash \mathrm{H}, 0,1.771796,6.423787,3.348685 \mathrm{IC}, 0,-0.3$ $54205,6.017998,3.627006 \mathrm{H}, 0,-0.478075,6.783429,4.400862 \mathrm{lC}, 0,-1.428008$ $5.22922,3.245268 \mathrm{lH}, 0,-2.408593,5.355821,3.71831 \mathrm{lC}, 0,-3.356291,1.423962$ $, 0.621108 \mathrm{IC}, 0,-3.755861,0.392987,1.518323 \backslash \mathrm{C}, 0,-4.041019,1.595248,-0.60$ $902 \backslash \mathrm{C}, 0,-4.840773,-0.424593,1.160731 \backslash \mathrm{C}, 0,-5.118109,0.741475,-0.92445 \backslash \mathrm{C}$ $, 0,-5.538746,-0.276251,-0.053492 \mathrm{H}, 0,-5.160246,-1.210433,1.855016 \mathrm{lH}, 0$, $-5.634674,0.889132,-1.878035 \backslash \mathrm{C}, 0,3.872257,0.014357,-0.830574 \backslash \mathrm{C}, 0,3.935$ $891,-0.780865,-2.005957 \backslash C, 0,4.384181,-0.488826,0.397494 \backslash C, 0,4.534807$,$2.053939,-1.931494 \backslash \mathrm{C}, 0,4.966817,-1.768214,0.420356 \mid \mathrm{C}, 0,5.057545,-2.568$ $05,-0.733937 \backslash \mathrm{H}, 0,4.593146,-2.669014,-2.837826 \backslash \mathrm{H}, 0,5.367708,-2.148658,1$ $.367376 \mathrm{IC}, 0,4.352481,0.337978,1.684681 \mathrm{lH}, 0,3.832692,1.28589,1.466463 \mathrm{IC}$ $, 0,3.384076,-0.29134,-3.347017 \backslash \mathrm{C}, 0,5.70709,-3.948943,-0.693092 \backslash \mathrm{H}, 0,5.6$ $6489,-4.355884,-1.720958 \mathrm{lC}, 0,4.529489,0.073515,-4.314423 \mathrm{H}, 0,5.203247$, $0.83126,-3.876298 \mathrm{H}, 0,5.138233,-0.816412,-4.560869 \mathrm{H}, 0,4.125415,0.4805$ $31,-5.259799 \backslash \mathrm{C}, 0,4.933916,-4.917921,0.224457 \backslash \mathrm{H}, 0,3.876801,-5.001589,-0$ $.083009 \backslash \mathrm{H}, 0,5.382339,-5.92782,0.19062 \mathrm{H}, 0,4.957248,-4.575659,1.275309 \backslash$ $\mathrm{C}, 0,7.191931,-3.864508,-0.283685 \mathrm{H}, 0,7.30056,-3.482589,0.748302 \backslash \mathrm{H}, 0,7$. $665738,-4.862549,-0.324549 \backslash \mathrm{H}, 0,7.753943,-3.190911,-0.955254 \backslash \mathrm{C}, 0,3.5625$ $51,-0.367966,2.804204 \mathrm{H}, 0,2.534858,-0.593714,2.475306 \backslash \mathrm{H}, 0,4.050325,-1$. $311918,3.106326 \backslash \mathrm{H}, 0,3.504125,0.277931,3.699732 \mathrm{CC}, 0,5.780053,0.701139,2$ $.144904 \mathrm{IH}, 0,5.746437,1.349391,3.039993 \mathrm{H}, 0,6.361233,-0.203123,2.404771$ $\mathrm{IH}, 0,6.329013,1.240735,1.352188 \backslash \mathrm{C}, 0,-6.696879,-1.228492,-0.365683 \mathrm{IH}, 0$, $-7.352776,-1.222909,0.527859 \backslash \mathrm{C}, 0,-3.057877,0.176328,2.863261 \mathrm{H}, 0,-2.06$ $2315,0.645083,2.787547 \backslash \mathrm{C}, 0,-3.655881,2.706413,-1.5881 \backslash \mathrm{H}, 0,-2.800953,3$. $252256,-1.154513 \backslash \mathrm{C}, 0,-7.550414,-0.804829,-1.570514 \backslash \mathrm{H}, 0,-6.972458,-0.84$ $8136,-2.51199 \mathrm{lH}, 0,-8.414159,-1.484254,-1.682112 \mathrm{lH}, 0,-7.9404,0.222417,-$ $1.456354 \backslash \mathrm{C}, 0,-6.19186,-2.679196,-0.542439 \backslash \mathrm{H}, 0,-5.612367,-3.01459,0.335$ $216 \mathrm{H}, 0,-7.043364,-3.372161,-0.672438 \mathrm{H}, 0,-5.542803,-2.76324,-1.431493$ IC, $0,-3.195523,2.148366,-2.948748 \mathrm{H}, 0,-2.839631,2.968267,-3.599457 \backslash \mathrm{H}, 0$ ,-2.368508,1.43032,-2.817944\H,0,-4.024321,1.642149,-3.476789\C,0,-4.8 $01259,3.725296,-1.757083 \mathrm{H}, 0,-5.097567,4.156079,-0.783674 \mathrm{H}, 0,-4.48831$ $3,4.5549,-2.417575 \backslash \mathrm{H}, 0,-5.695347,3.255764,-2.2075 \backslash \mathrm{C}, 0,-3.837346,0.8629$ $86,4.005886 \mathrm{H}, 0,-4.842902,0.41549,4.117454 \mathrm{IH}, 0,-3.303744,0.743866,4.96$ $7104 \mathrm{IH}, 0,-3.969523,1.942804,3.821961 \backslash \mathrm{C}, 0,-2.828442,-1.312272,3.187805 \backslash$ $\mathrm{H}, 0,-2.212493,-1.407995,4.100999 \mathrm{H}, 0,-3.776936,-1.849073,3.375785 \mathrm{lH}, 0$, $-2.288774,-1.803359,2.364115 \mathrm{IC}, 0,2.412359,-1.302051,-3.987536 \mathrm{IH}, 0,1.99$ $6332,-0.884627,-4.923432 \mathrm{H}, 0,2.916327,-2.251664,-4.246167 \backslash \mathrm{H}, 0,1.576813$ ,-1.511465,-3.299229) $, 0,2.806208,0.627495,-3.150008 \mathrm{IC}, 0,1.657311,-4.0$ $19332,3.230933 \backslash \mathrm{C}, 0,0.403281,-4.52914,3.634918 \backslash \mathrm{H}, 0,0.278225,-4.906442,4$ $.655821 \backslash \mathrm{C}, 0,-0.682356,-4.556741,2.758265 \mathrm{lH}, 0,-1.641896,-4.942817,3.112$ $657 \backslash \mathrm{C}, 0,-0.556275,-4.051905,1.440468 \backslash \mathrm{C}, 0,0.704088,-3.543906,1.031763 \backslash \mathrm{H}$ $, 0,0.850396,-3.17452,0.01168 \backslash \mathrm{C}, 0,1.78564,-3.538669,1.909756 \backslash \mathrm{H}, 0,2.7476$ $45,-3.150587,1.563762 \backslash \mathrm{C}, 0,-1.68594,-4.055099,0.509686 \mathrm{IC}, 0,-2.809261,-5$ $.031415,0.654472 \mathrm{H}, 0,-3.098234,-5.092764,1.716474 \backslash \mathrm{H}, 0,-2.481938,-6.042$ 
$454,0.352023 \mathrm{WH}, 0,-3.687362,-4.740816,0.060796 \mathrm{IN}, 0,-1.674842,-3.156892$ $-0.460701 \mathrm{H}, 0,-0.99934,-2.371548,-0.349809 \mathrm{CC}, 0,-2.491374,-3.064009,-1$. $623627 \backslash \mathrm{C}, 0,-2.829072,-4.208652,-2.371927 \backslash \mathrm{H}, 0,-2.472392,-5.195044,-2.06$ $4227 \backslash \mathrm{C}, 0,-3.590175,-4.066364,-3.538836 \mathrm{H}, 0,-3.84923,-4.955711,-4.12266$ $2 \mathrm{IC}, 0,-3.997922,-2.793293,-3.968148 \mathrm{IC}, 0,-3.64055,-1.65731,-3.224696 \mathrm{IH}$ $0,-3.957469,-0.66345,-3.551094 \mathrm{IC}, 0,-2.888572,-1.783083,-2.051299 \mathrm{lH}, 0$,$2.632593,-0.900527,-1.460074 \mathrm{IH}, 0,-4.587432,-2.685425,-4.884529 \mathrm{IC}, 0,2.8$ $18392,-3.959398,4.189892 \mathrm{H}, 0,2.769065,-4.767088,4.939662 \backslash \mathrm{H}, 0,3.782777$, $-4.020001,3.658454|\mathrm{H}, 0,2.80362,-2.998071,4.738813| \mid \mathrm{V} e r s i o n=E S 64 L-G 09 R e$ vD.01\State=1-AlHF=-3216.0861985 $\mathrm{RMSD}=4.937 \mathrm{e}-09 \mathrm{IDipole}=-1.8123446,-4.4$ $787249,0.96116021$ Quadrupole $=-7.8820823,22.2282103,-14.3461281,16.69278$ 15,10.0588739,-7.6403088IPG=C01 [X(C65H72N1O4P1)]॥@

\section{TRIP/2E Type I}

Confo

1\1\GINC-WORKER0ISPIRTPSSTPSSIdef2SVPIC65H69F3N1O4P1\JHIOEI07-May-2019

IOI\#p tpsstpss/def2svp int=ultrafine empiricaldispersion=gd3 scrf=(sm $\mathrm{d}$, solvent=ch2cl2, read,externaliteration, dovacuum) \titlel|0,1\P,0,0.19 $0648,-0.599056,-1.387063 \backslash 0,0,-1.128743,0.348037,-1.786618 \backslash 0,0,0.968308$ $, 0.458384,-0.31239910,0,0.959487,-0.804237,-2.658069 \backslash 0,0,-0.219947,-1$ $741798,-0.460601 \backslash \mathrm{C}, 0,-1.235505,2.217502,-0.291974 \backslash \mathrm{C}, 0,-1.819331,1.0688$ $67,-0.834822 \backslash \mathrm{C}, 0,-3.151846,0.646063,-0.528005 \backslash \mathrm{C}, 0,-3.877778,1.401982,0$ $.38302 \backslash \mathrm{H}, 0,-4.907586,1.115694,0.621441 \backslash \mathrm{C}, 0,-3.308486,2.526644,1.046955$ IC,0,-1.959628,2.931552,0.732511\C,0,-1.382377,3.995808,1.489673\H,0,$0.345155,4.285256,1.298847 \backslash \mathrm{C}, 0,-2.108892,4.651208,2.473021 \backslash \mathrm{H}, 0,-1.6386$ $51,5.456918,3.047496 \mathrm{IC}, 0,-3.4525,4.281289,2.747339 \mathrm{IH}, 0,-4.015859,4.808$ $435,3.524856 \mathrm{IC}, 0,-4.035446,3.236125,2.049659 \backslash \mathrm{H}, 0,-5.058803,2.917101,2$. $275834 \backslash \mathrm{C}, 0,0.115085,2.657764,-0.746825 \backslash \mathrm{C}, 0,1.191735,1.766732,-0.692677$ IC $, 0,2.546155,2.162107,-0.922713 \backslash \mathrm{C}, 0,2.783625,3.473773,-1.308423 \backslash \mathrm{H}, 0,3$ $.812509,3.811606,-1.469607 \backslash \mathrm{C}, 0,1.718568,4.399649,-1.507265 \backslash \mathrm{C}, 0,0.36162$ $3,3.993339,-1.233828 \mathrm{IC}, 0,-0.686074,4.930548,-1.484625 \backslash \mathrm{H}, 0,-1.723903,4$. $626345,-1.320906 \backslash \mathrm{C}, 0,-0.408344,6.208754,-1.946547 \backslash \mathrm{H}, 0,-1.2308,6.906701$ ,-2.138303।C $, 0,0.931772,6.618026,-2.180498 \mathrm{H}, 0,1.138282,7.632498,-2.53$ $8703 \backslash \mathrm{C}, 0,1.971389,5.725923,-1.970578 \mathrm{lH}, 0,3.008575,6.0221,-2.165085 \mathrm{IC}, 0$ ,-1.840767,-0.32622,4.222974\C,0,-1.411562,-1.484586,4.888998\H,0,-1.9 $03548,-1.804252,5.811275 \backslash \mathrm{C}, 0,-0.349623,-2.230366,4.366465 \mathrm{IH}, 0,-0.02470$ $7,-3.135077,4.887599 \backslash \mathrm{C}, 0,0.264,-1.842085,3.154865 \backslash \mathrm{C}, 0,-0.168735,-0.665$ $363,2.502005 \mathrm{IH}, 0,0.321572,-0.321303,1.588773 \mathrm{IC}, 0,-1.211234,0.090163,3$. $037115 \mathrm{lH}, 0,-1.535486,1.00229,2.529181 \backslash \mathrm{C}, 0,1.361662,-2.659383,2.593705$ $\mathrm{C}, 0,2.321206,-3.336595,3.522438 \backslash \mathrm{H}, 0,3.295159,-3.50816,3.038505 \backslash \mathrm{H}, 0,1.9$ $16938,-4.316567,3.836945 \mathrm{H}, 0,2.454501,-2.72451,4.427382 \mathrm{VN}, 0,1.469968,-$ $2.736667,1.289386 \backslash \mathrm{H}, 0,0.742524,-2.238373,0.65165 \backslash \mathrm{C}, 0,2.427017,-3.47187$ $7,0.520809 \backslash \mathrm{C}, 0,3.003219,-2.823454,-0.587703 \backslash \mathrm{H}, 0,2.779532,-1.773753,-0$. $794647 \backslash \mathrm{C}, 0,3.890291,-3.529497,-1.408809 \backslash \mathrm{H}, 0,4.34505,-3.019928,-2.26317$ IC,0,4.199673,-4.870454,-1.132929|C,0,3.612944,-5.512044,-0.030378|H,0 $3.838747,-6.562245,0.181649 \backslash \mathrm{C}, 0,2.718924,-4.820902,0.79776 \mathrm{IH}, 0,2.2266$ $87,-5.333471,1.628055 \backslash C, 0,3.659037,1.189295,-0.670731 \backslash \mathrm{C}, 0,4.352209,0.5$ $83344,-1.749856 \mid \mathrm{C}, 0,4.012893,0.877035,0.673051 \mathrm{IC}, 0,5.405428,-0.307483$ $-1.461655 \backslash \mathrm{C}, 0,5.063731,-0.025715,0.906091 \backslash \mathrm{C}, 0,5.773365,-0.632236,-0.14$ $6852 \backslash \mathrm{H}, 0,5.947451,-0.779771,-2.289265 \mathrm{lH}, 0,5.338491,-0.264013,1.938324$ C, $0,-3.729094,-0.562236,-1.200017 \backslash \mathrm{C}, 0,-4.029362,-1.729752,-0.446566 \mid C$, $0,-3.981801,-0.540356,-2.601831 \backslash \mathrm{C}, 0,-4.585964,-2.841793,-1.109176 \backslash \mathrm{C}, 0$, $-4.534579,-1.677918,-3.213602 \backslash \mathrm{C}, 0,-4.845256,-2.841957,-2.487905 \backslash \mathrm{H}, 0,-4$ $.817012,-3.744788,-0.530598 \mathrm{H}, 0,-4.733014,-1.652777,-4.291564 \backslash \mathrm{H}, 0,4.89$ $4103,-5.417168,-1.779403 \backslash \mathrm{C}, 0,-2.996181,0.489098,4.74941 \backslash \mathrm{C}, 0,-3.7145,0$. $697306,-3.462025 \mathrm{IH}, 0,-3.25376,1.467185,-2.821047 \backslash \mathrm{C}, 0,-3.749299,-1.8373$ $85,1.05382 \mathrm{IC}, 0,-5.438411,-4.068184,-3.17656 \mathrm{lH}, 0,-5.586646,-4.838385,-2$ $.396343 \mid \mathrm{C}, 0,-5.058437,-1.92374,1.864656 \mathrm{WH}, 0,-4.844969,-1.978576,2.9470$ $28 \mathrm{H}, 0,-5.69833,-1.040966,1.688183 \backslash \mathrm{H}, 0,-5.638966,-2.82354,1.588486 \backslash \mathrm{C}, 0$ $,-4.470569,-4.64781,-4.228872 \backslash \mathrm{H}, 0,-4.288688,-3.924682,-5.045211 \backslash \mathrm{H}, 0,-3$ $.49452,-4.900695,-3.777744 \backslash \mathrm{H}, 0,-4.888957,-5.566424,-4.68001 \backslash \mathrm{C}, 0,-6.815$ $776,-3.761287,-3.799602 \backslash \mathrm{H}, 0,-7.253645,-4.673354,-4.245347 \backslash \mathrm{H}, 0,-7.52154$ $2,-3.377969,-3.040877 \backslash \mathrm{H}, 0,-6.733175,-3.00231,-4.599533 \backslash \mathrm{C}, 0,-2.725395,0$ $.402374,-4.608182 \mathrm{H}, 0,-3.14347,-0.327662,-5.325828 \mathrm{H}, 0,-2.501505,1.328$ $896,-5.168535 \mathrm{IH}, 0,-1.776451,0.001859,-4.214463 \mathrm{IC}, 0,-5.03397,1.291492,-$ $3.998987 \backslash \mathrm{H}, 0,-4.837824,2.218726,-4.568504 \mathrm{IH}, 0,-5.548725,0.583509,-4.67$ $4495 \mathrm{IH}, 0,-5.726085,1.537107,-3.173236 \backslash \mathrm{C}, 0,6.919963,-1.603488,0.122168 \mathrm{I}$ $\mathrm{H}, 0,7.123984,-2.130401,-0.829283 \backslash \mathrm{C}, 0,3.987681,0.885684,-3.204212 \mathrm{H}, 0,2$ $.990965,1.359376,-3.196844 \backslash \mathrm{C}, 0,3.299496,1.549604,1.849073 \backslash \mathrm{H}, 0,2.255573$ $, 1.733762,1.540852 \mathrm{CC}, 0,8.201439,-0.8381,0.516324 \mathrm{IH}, 0,9.045477,-1.53594$ $2,0.668679 \mathrm{|H}, 0,8.490434,-0.114725,-0.26684 \backslash \mathrm{H}, 0,8.049393,-0.276974,1.45$ $7175 \backslash \mathrm{C}, 0,6.565618,-2.669149,1.177847 \backslash \mathrm{H}, 0,6.425515,-2.220902,2.17847 \backslash \mathrm{H}$ $0,5.638119,-3.204747,0.909755 \mathrm{IH}, 0,7.379221,-3.412605,1.259663 \mathrm{IC}, 0,3.24$ $6036,0.681159,3.117744 \backslash \mathrm{H}, 0,2.876225,-0.333317,2.893408 \mathrm{H}, 0,4.237567,0$. $583409,3.596178 \mathrm{IH}, 0,2.565893,1.135842,3.859646 \mathrm{IC}, 0,3.935759,2.923263,2$ 
155403\H,0,3.917824,3.580376,1.268542\H,0,3.389145,3.433154,2.970107\ $\mathrm{H}, 0,4.988897,2.803109,2.470059 \mathrm{lC}, 0,4.989169,1.886242,-3.819271 \mathrm{lH}, 0,5.0$ $32813,2.82437,-3.238802 \backslash \mathrm{H}, 0,6.008305,1.456959,-3.843238 \backslash \mathrm{H}, 0,4.701643,2$ $138688,-4.856671 \backslash \mathrm{C}, 0,3.873197,-0.383639,-4.070038 \mathrm{lH}, 0,3.531704,-0.112$ $802,-5.086175 \mathrm{IH}, 0,4.843729,-0.901641,-4.178079 \mathrm{IH}, 0,3.133442,-1.076725$, $-3.638535 \backslash \mathrm{C}, 0,-2.81164,-3.018736,1.377231 \backslash \mathrm{H}, 0,-3.286722,-3.991075,1.15$ $0501 \backslash \mathrm{H}, 0,-1.879085,-2.937932,0.79484 \backslash \mathrm{H}, 0,-2.554352,-3.015583,2.452041 \backslash$ $\mathrm{H}, 0,-3.226223,-0.91917,1.365743 \mathrm{IF}, 0,-2.647595,1.786384,4.953087 \backslash \mathrm{F}, 0,-4$ $.035356,0.505682,3.866125 \mathrm{IF}, 0,-3.484097,0.017696,5.92033$ IIVersion=ES64 L-G09RevD.01।State=1-AlHF=-3513.6183668IRMSD=2.003e-09lDipole=2.580562 $6,-2.8086783,4.6444803 \backslash$ Quadrupole $=-7.9075191,17.3759406,-9.4684214,-16$ .2257663,8.8167536,-23.2629552/PG=C01 [X(C65H69F3N1O4P1)]॥@

Conf1

1\1\GINC-WORKER1ISPIRTPSSTPSSIdef2SVPIC65H69F3N1O4P1\JHIOEI07-May-2019 IOl॥ $\mathrm{p}$ tpstpss/def2svp int=ultrafine empiricaldispersion=gd3 scrf $=(\mathrm{sm}$ $\mathrm{d}$, solvent=ch2cl2, read,externaliteration, dovacuum) \titlel|0,1\P, $0,0.20$ $0875,-0.478127,-1.498589 \backslash 0,0,-1.097342,0.512456,-1.861386 \backslash 0,0,0.970199$ $, 0.495047,-0.34057210,0,0.995901,-0.61238,-2.76339910,0,-0.245701,-1.6$ $73391,-0.658898 \mid C, 0,-1.18756,2.298214,-0.268917 \backslash \mathrm{C}, 0,-1.785186,1.189899$ $,-0.875556 \backslash \mathrm{C}, 0,-3.123704,0.768258,-0.593491 \backslash \mathrm{C}, 0,-3.840443,1.481657,0.3$ $5866 \mathrm{IH}, 0,-4.87257,1.193495,0.584258 \mathrm{IC}, 0,-3.258698,2.562865,1.081292 \mathrm{IC}$, $0,-1.904971,2.967818,0.789167 \backslash \mathrm{C}, 0,-1.314567,3.983284,1.60116 \backslash \mathrm{H}, 0,-0.27$ $3785,4.269614,1.425038 \mathrm{CC}, 0,-2.032821,4.593562,2.618932 \backslash \mathrm{H}, 0,-1.553443,5$ $.362087,3.235233 \mathrm{IC}, 0,-3.380332,4.224512,2.87466 \mathrm{IH}, 0,-3.937023,4.715295$ $3.680244 \mathrm{IC}, 0,-3.976276,3.225593,2.122356 \mathrm{IH}, 0,-5.003307,2.907269,2.332$ $214 \mid C, 0,0.178679,2.73197,-0.679857 \backslash C, 0,1.23408,1.8163,-0.640458 \backslash C, 0,2$. $6038,2.19196,-0.803646 \backslash \mathrm{C}, 0,2.879591,3.512749,-1.126971 \backslash \mathrm{H}, 0,3.919351,3$ $836141,-1.240988 \mid C, 0,1.838866,4.468232,-1.319241 \backslash C, 0,0.466386,4.081804$ ,-1.097963।C $, 0,-0.554742,5.052041,-1.331083 \backslash \mathrm{H}, 0,-1.602281,4.763357,-1$. 2041111C $, 0,-0.238509,6.341478,-1.73329 \mathrm{IH}, 0,-1.040563,7.065612,-1.91399$ IC, $0,1.115485,6.728845,-1.920248 \backslash \mathrm{H}, 0,1.35254,7.751828,-2.232273 \backslash \mathrm{C}, 0,2$. $130624,5.806131,-1.722216 \backslash \mathrm{H}, 0,3.178351,6.087305,-1.879013 \backslash \mathrm{C}, 0,-1.85464$ $6,-0.475044,4.129338 \mathrm{IC}, 0,-1.433846,-1.670158,4.733017 \backslash \mathrm{H}, 0,-1.921972,-2$ $.02939,5.642599 \mathrm{CC}, 0,-0.385379,-2.401096,4.164335 \mathrm{H}, 0,-0.065093,-3.3330$ $61,4.637908 \backslash \mathrm{C}, 0,0.220679,-1.961984,2.966241 \backslash \mathrm{C}, 0,-0.204243,-0.75043,2.3$ $75517 \backslash \mathrm{H}, 0,0.281395,-0.368611,1.475103 \backslash \mathrm{C}, 0,-1.23043,-0.008632,2.959284 \backslash$ $\mathrm{H}, 0,-1.546536,0.931794,2.500323 \mathrm{IC}, 0,1.302314,-2.768354,2.358973 \mathrm{IC}, 0,2$. $262913,-3.494661,3.2496 \mathrm{H}, 0,3.231634,-3.650095,2.749523 \backslash \mathrm{H}, 0,1.855171$,$4.485127,3.523519 \backslash \mathrm{H}, 0,2.408149,-2.925043,4.180033 \backslash \mathrm{N}, 0,1.396878,-2.7985$ $36,1.051876 \backslash \mathrm{H}, 0,0.68173,-2.255608,0.435964 \backslash \mathrm{C}, 0,2.335528,-3.526719,0.25$ $4158 \mathrm{IC}, 0,2.932618,-2.853057,-0.827604 \backslash \mathrm{H}, 0,2.734265,-1.792258,-0.999415$ IC $, 0,3.808592,-3.550261,-1.668289 \backslash \mathrm{H}, 0,4.280286,-3.021967,-2.501798 \mid \mathrm{C}, 0$ , 4.08446,-4.907183,-1.439404IC,0,3.47409,-5.574561,-0.365335।H,0,3.673 $115,-6.636948,-0.190578 \backslash \mathrm{C}, 0,2.592053,-4.892033,0.482079 \backslash \mathrm{H}, 0,2.084234$,$5.421075,1.292372 \mathrm{IC}, 0,3.677126,1.178141,-0.541044 \mathrm{IC}, 0,4.395511,0.58249$ $5,-1.60878|C, 0,3.954318,0.804129,0.805926| C, 0,5.38773,-0.372519,-1.307$ 433।C $, 0,4.944978,-0.160564,1.051051 \backslash \mathrm{C}, 0,5.669797,-0.768155,0.00879 \mathrm{IH}, 0$ $, 5.943929,-0.841264,-2.127337 \backslash \mathrm{H}, 0,5.159782,-0.449526,2.084509 \backslash \mathrm{C}, 0,-3.7$ $12934,-0.396096,-1.329875 \backslash \mathrm{C}, 0,-4.021046,-1.603069,-0.641149 \backslash \mathrm{C}, 0,-3.963$ $894,-0.295761,-2.726614 \backslash \mathrm{C}, 0,-4.581217,-2.67338,-1.361811 \backslash \mathrm{C}, 0,-4.52359$, $-1.397527,-3.399943 \mathrm{IC}, 0,-4.838612,-2.596883,-2.741231 \backslash \mathrm{H}, 0,-4.814493,-3$ $600796,-0.826029 \backslash \mathrm{H}, 0,-4.724026,-1.319488,-4.475684 \backslash \mathrm{H}, 0,4.770459,-5.44$ $6367,-2.1010291 \mathrm{C}, 0,-2.996585,0.326635,4.7043521 \mathrm{C}, 0,-3.687326,0.986128$, $-3.515415 \mathrm{IH}, 0,-3.217978,1.712849,-2.831449 \mathrm{IC}, 0,-3.751628,-1.791627,0.8$ $5338 \backslash \mathrm{C}, 0,-5.426006,-3.781098,-3.504217 \mathrm{WH}, 0,-5.554897,-3.458339,-4.5546$ $11 \mathrm{IC}, 0,-5.068406,-1.881847,1.651256 \mathrm{H}, 0,-4.863912,-1.984342,2.731858 \mathrm{H}$ $, 0,-5.68708,-0.978615,1.505494 \backslash \mathrm{H}, 0,-5.666533,-2.756595,1.334575 \backslash \mathrm{C}, 0,-6$ $.813633,-4.181439,-2.963568 \mathrm{H}, 0,-6.748736,-4.536727,-1.918532 \backslash \mathrm{H}, 0,-7.5$ $14692,-3.32789,-2.989164 \backslash \mathrm{H}, 0,-7.246189,-4.998617,-3.569498 \mathrm{IC}, 0,-4.4613$ $78,-4.985638,-3.499897 \backslash \mathrm{H}, 0,-4.871203,-5.815648,-4.104477 \backslash \mathrm{H}, 0,-3.476335$ $,-4.710393,-3.91711 \mathrm{lH}, 0,-4.300932,-5.363294,-2.473053 \mathrm{IC}, 0,-2.702368,0$. $752166,-4.679038 \mathrm{H}, 0,-3.12654,0.070756,-5.43941 \backslash \mathrm{H}, 0,-2.471641,1.709514$ $,-5.181764 \mathrm{IH}, 0,-1.756114,0.321113,-4.311801 \mathrm{IC}, 0,-5.003231,1.622153,-4$. $011796 \mathrm{HH}, 0,-4.80084,2.577756,-4.530009 \mathrm{H}, 0,-5.528873,0.95805,-4.722511$ $\backslash \mathrm{H}, 0,-5.688327,1.828242,-3.169577 \backslash \mathrm{C}, 0,6.738523,-1.818924,0.298118 \mathrm{H}, 0$, $6.995168,-2.291614,-0.668758 \mathrm{IC}, 0,4.119501,0.962683,-3.064479 \mathrm{IH}, 0,3.139$ $59,1.469768,-3.08672 \mathrm{CC}, 0,3.227662,1.478181,1.973222 \mathrm{H}, 0,2.198678,1.700$ $782,1.63969 \backslash \mathrm{C}, 0,8.019502,-1.160714,0.853573 \backslash \mathrm{H}, 0,8.811091,-1.915358,1.0$ $16186 \mathrm{lH}, 0,8.4103,-0.397745,0.156779 \mathrm{lH}, 0,7.818667,-0.664428,1.821308 \mathrm{C}$ $0,6.233466,-2.929215,1.241344 \backslash \mathrm{H}, 0,6.016294,-2.535015,2.250892 \mathrm{H}, 0,5.31$ $3501,-3.397323,0.850277 \backslash \mathrm{H}, 0,6.998843,-3.718762,1.35195 \backslash \mathrm{C}, 0,3.114002,0$. $595803,3.228194 \backslash \mathrm{H}, 0,2.721159,-0.404985,2.983961 \mathrm{WH}, 0,4.089184,0.464842$, $3.731898 \mathrm{VH}, 0,2.427137,1.062025,3.956608 \mathrm{IC}, 0,3.898165,2.826202,2.321067$ $\mathrm{IH}, 0,3.926636,3.499374,1.446843 \backslash \mathrm{H}, 0,3.345556,3.338899,3.129944 \backslash \mathrm{H}, 0,4.9$ $37598,2.666139,2.662268 \mathrm{CC}, 0,5.183624,1.955666,-3.578892 \mathrm{H}, 0,5.228303,2$ $.863971,-2.952747 \backslash \mathrm{H}, 0,6.188315,1.493042,-3.573123 \backslash \mathrm{H}, 0,4.957409,2.26578$ 
4,-4.615768\C,0,4.010091,-0.259601,-3.995371\H,0,3.732366,0.069049,-5. $013843 \mathrm{H}, 0,4.96732,-0.806559,-4.078072 \mathrm{H}, 0,3.225816,-0.945552,-3.63722$ $1 \mathrm{CC}, 0,-2.848787,-3.0126,1.124501 \mathrm{H}, 0,-3.345342,-3.959161,0.841396 \mathrm{H}, 0$, $-1.907168,-2.926823,0.557512 \backslash \mathrm{H}, 0,-2.605256,-3.074171,2.200685 \backslash \mathrm{H}, 0,-3.2$ $07155,-0.903912,1.213586 \mathrm{~F}, 0,-2.628395,1.60436,4.984289 \mathrm{IF}, 0,-3.493028$, $-0.205476,5.845489$ IF $, 0,-4.034666,0.412252,3.823795$ IIVersion=ES64L-G09R evD.01 IState=1-AlHF=-3513.6185302/RMSD=1.979e-09|Dipole=2.4812717,-3.1 $217463,4.4842739$ lQuadrupole $=-8.3960565,20.8546422,-12.4585856,-15.9093$ 918,7.5129681,-20.8432461|PG=C01 [X(C65H69F3N1O4P1)]॥@

\section{Conf2}

1।1।GINC-WORKER2ISPIRTPSSTPSSIdef2SVPIC65H69F3N1O4P1 IJHIOEI07-May-2019

IO\\\# tpsstpss/def2svp int=ultrafine empiricaldispersion=gd3 scrf=(sm $\mathrm{d}$,solvent=ch2cl2, read,externaliteration, dovacuum) \titlell0,1\P,0,0.24 $8848,-0.6155,-1.398916 \backslash 0,0,-0.979716,0.398412,-1.90755910,0,0.999978,0$ $.402265,-0.26567 \backslash 0,0,1.104995,-0.875547,-2.602143 \backslash 0,0,-0.305698,-1.719$ $652,-0.501274|C, 0,-1.108829,2.273267,-0.426609| C, 0,-1.706501,1.152367$, $-1.009425 \backslash \mathrm{C}, 0,-3.075015,0.792397,-0.795595 \backslash \mathrm{C}, 0,-3.827242,1.589129,0.05$ $7572 \backslash \mathrm{H}, 0,-4.882976,1.350768,0.224584 \mathrm{CC}, 0,-3.25543,2.691786,0.754941 \backslash \mathrm{C}$, $0,-1.869898,3.031296,0.537186 \backslash \mathrm{C}, 0,-1.30077,4.076854,1.326119 \backslash \mathrm{H}, 0,-0.24$ $0739,4.319125,1.208238 \mathrm{IC}, 0,-2.065499,4.773318,2.250593 \mathrm{lH}, 0,-1.601898,5$ $.563441,2.851506 \mathrm{IC}, 0,-3.440275,4.465482,2.431368 \mathrm{lH}, 0,-4.032738,5.02353$ $3,3.164389 \backslash \mathrm{C}, 0,-4.018901,3.441344,1.699693 \backslash \mathrm{H}, 0,-5.068988,3.170008,1.85$ $4153|C, 0,0.294111,2.638156,-0.776978| C, 0,1.318084,1.696395,-0.627258 \mid C$ $, 0,2.703353,2.028231,-0.740742 \mathrm{lC}, 0,3.032524,3.322775,-1.120036 \mathrm{VH}, 0,4.0$ $86313,3.610948,-1.193654|C, 0,2.033524,4.291262,-1.42588| C, 0,0.641439,3$ $.951827,-1.26008 \mathrm{IC}, 0,-0.338077,4.928383,-1.614387 \backslash \mathrm{H}, 0,-1.398773,4.6742$ $96,-1.53053|\mathrm{C}, 0,0.035532,6.181512,-2.077383| \mathrm{H}, 0,-0.735152,6.910297,-2$. $351776 \backslash \mathrm{C}, 0,1.408027,6.525367,-2.206617 \backslash \mathrm{H}, 0,1.689964,7.520847,-2.566607$ IC $, 0,2.384767,5.594463,-1.890517 \backslash \mathrm{H}, 0,3.447038,5.840607,-2.00098 \mid C, 0,-2$ $.169444,-0.153943,4.113601 \backslash \mathrm{C}, 0,-1.849614,-1.32973,4.810084 \backslash \mathrm{H}, 0,-2.4119$ $16,-1.610114,5.704465 \backslash \mathrm{C}, 0,-0.806938,-2.142893,4.353564 \backslash \mathrm{H}, 0,-0.568987,-$ $3.06172,4.896682 \backslash C, 0,-0.102929,-1.80344,3.176944 \backslash C, 0,-0.425747,-0.6085$ $13,2.493256 \mathrm{IH}, 0,0.134591,-0.303409,1.606473 \mathrm{IC}, 0,-1.449254,0.213336,2.9$ $63483 \mathrm{IH}, 0,-1.688118,1.13909,2.432858 \mathrm{IC}, 0,0.969944,-2.693104,2.682642 \mathrm{IC}$ $, 0,1.810169,-3.452907,3.660895 \mathrm{H}, 0,2.786416,-3.720564,3.228535 \mathrm{VH}, 0,1.2$ $96972,-4.387556,3.953602 \backslash \mathrm{H}, 0,1.953831,-2.852599,4.572306 \mathrm{lN}, 0,1.166964$, $-2.762127,1.389375 \mathrm{IH}, 0,0.518714,-2.222582,0.699369 \mathrm{IC}, 0,2.125687,-3.553$ $082,0.679704 \backslash \mathrm{C}, 0,2.867468,-2.916209,-0.332845 \mathrm{IH}, 0,2.771181,-1.839402,-$ $0.495931 \backslash \mathrm{C}, 0,3.748358,-3.674368,-1.112456 \backslash \mathrm{H}, 0,4.331117,-3.178094,-1.89$ $3445 \backslash \mathrm{C}, 0,3.88748,-5.053557,-0.889771 \backslash \mathrm{C}, 0,3.139558,-5.680366,0.119151 \mathrm{IH}$ $, 0,3.234965,-6.757897,0.288984 \backslash \mathrm{C}, 0,2.249445,-4.936183,0.905379 \backslash \mathrm{H}, 0,1.6$ $3284,-5.427553,1.662815 \backslash \mathrm{C}, 0,3.765467,1.032944,-0.377182 \backslash \mathrm{C}, 0,4.564284,0$ $.424773,-1.381264 \backslash \mathrm{C}, 0,4.007002,0.748641,0.995947 \backslash \mathrm{C}, 0,5.630305,-0.40922$ $9,-0.990961 \backslash \mathrm{C}, 0,5.083037,-0.091916,1.332939 \backslash \mathrm{C}, 0,5.921845,-0.663775,0.3$ $60139 \mathrm{lH}, 0,6.255375,-0.863993,-1.767802 \backslash \mathrm{H}, 0,5.293241,-0.297839,2.388413$ $\mid \mathrm{C}, 0,-3.659712,-0.401406,-1.485848 \backslash \mathrm{C}, 0,-4.066686,-1.537694,-0.730557 \backslash \mathrm{C}$ $, 0,-3.8144,-0.400707,-2.899651 \backslash \mathrm{C}, 0,-4.630077,-2.635983,-1.405078 \mid \mathrm{C}, 0,-$ $4.377112,-1.528735,-3.52542 \mathrm{IC}, 0,-4.792863,-2.657328,-2.800713 \mathrm{lH}, 0,-4.9$ $42846,-3.507929,-0.818744 \backslash \mathrm{H}, 0,-4.502544,-1.52682,-4.615222 \mathrm{H}, 0,4.57787$ $4,-5.640133,-1.50485 \backslash C, 0,-3.307545,0.729424,4.562902 \backslash \mathrm{C}, 0,-3.433928,0.8$ $05695,-3.76088 \mathrm{H}, 0,-2.97439,1.561941,-3.103102 \backslash \mathrm{C}, 0,-3.893868,-1.624933$ $, 0.787291 \backslash \mathrm{C}, 0,-5.401504,-3.865277,-3.507708 \backslash \mathrm{H}, 0,-5.397743,-3.641903,-4$ $.591193 \backslash \mathrm{C}, 0,-5.256188,-1.617021,1.509855 \mathrm{lH}, 0,-5.116914,-1.660611,2.604$ $648 \mathrm{H}, 0,-5.829904,-0.702637,1.275747 \backslash \mathrm{H}, 0,-5.869913,-2.48735,1.211699 \mathrm{C}$ $, 0,-6.866906,-4.092791,-3.081474 \backslash \mathrm{H}, 0,-6.935754,-4.334549,-2.004674 \backslash \mathrm{H}, 0$ $,-7.481808,-3.193972,-3.267206 \mathrm{IH}, 0,-7.311192,-4.934186,-3.64454 \mathrm{IC}, 0,-4$ $.557315,-5.13787,-3.288351 \backslash \mathrm{H}, 0,-4.976098,-5.986334,-3.860117 \backslash \mathrm{H}, 0,-3.51$ $2883,-4.985899,-3.614097 \backslash \mathrm{H}, 0,-4.540827,-5.426935,-2.221281 \backslash \mathrm{C}, 0,-2.3931$ $84,0.443342,-4.839381 \backslash \mathrm{H}, 0,-2.803877,-0.271953,-5.575759 \backslash \mathrm{H}, 0,-2.082852$, $1.349223,-5.392287 \backslash \mathrm{H}, 0,-1.494764,-0.003116,-4.381565 \backslash \mathrm{C}, 0,-4.687711,1.4$ $56049,-4.38328 \mathrm{H}, 0,-4.411961,2.361246,-4.95521 \mathrm{H}, 0,-5.199917,0.762556$, $-5.075541 \mathrm{~W}, 0,-5.410897,1.752604,-3.602242 \mathrm{lC}, 0,7.109854,-1.528895,0.77$ $6893 \mathrm{lH}, 0,7.182684,-1.461566,1.878883 \mathrm{IC}, 0,4.305041,0.678184,-2.86776 \mathrm{IH}$, $0,3.319659,1.168476,-2.948203 \backslash \mathrm{C}, 0,3.163294,1.396587,2.097355 \backslash \mathrm{H}, 0,2.154$ $281,1.564839,1.683746 \backslash \mathrm{C}, 0,6.895238,-3.01307,0.417973 \backslash \mathrm{H}, 0,7.747525,-3.6$ $24657,0.767489 \mathrm{H}, 0,5.975894,-3.407468,0.884225 \mathrm{H}, 0,6.807543,-3.149877$, $-0.675099 \mid \mathrm{C}, 0,8.434137,-1.002244,0.188969) \mathrm{H}, 0,8.441177,-1.074068,-0.91$ $4159 \mathrm{H}, 0,8.60005,0.055655,0.461031 \backslash \mathrm{H}, 0,9.289193,-1.591758,0.567436 \backslash \mathrm{C}, 0$

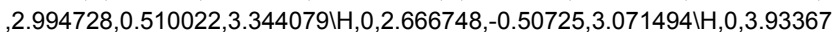
$3,0.421141,3.920129 \mathrm{IH}, 0,2.234758,0.944575,4.017576 \mathrm{IC}, 0,3.735356,2.7777$ $98,2.484207 \mathrm{VH}, 0,3.790411,3.448525,1.609064 \mathrm{H}, 0,3.098236,3.262203,3.246$ $995 \mathrm{IH}, 0,4.754408,2.675193,2.900902 \backslash \mathrm{C}, 0,5.364675,1.634076,-3.455026 \mathrm{H}, 0$ $, 5.390304,2.593137,-2.908529 \backslash \mathrm{H}, 0,6.374428,1.186341,-3.397803 \backslash \mathrm{H}, 0,5.148$ $166,1.850939,-4.517348 \mathrm{IC}, 0,4.220822,-0.625855,-3.684132 \mathrm{H}, 0,3.978858$,$0.393235,-4.737593 \backslash \mathrm{H}, 0,5.177651,-1.179557,-3.680524 \backslash \mathrm{H}, 0,3.421334,-1.27$ $3975,-3.290858 \mathrm{IC}, 0,-3.052607,-2.851372,1.195702 \backslash \mathrm{H}, 0,-3.571846,-3.79909$ 
$2,0.961327 \backslash \mathrm{H}, 0,-2.08375,-2.842064,0.670144 \backslash \mathrm{H}, 0,-2.862149,-2.83585,2.28$ 4116।H,0,-3.339262,-0.731706,1.117092IF,0,-3.872152,0.312726,5.71991/F $, 0,-4.30122,0.770054,3.629369 \mathrm{IF}, 0,-2.907349,2.013807,4.751115 \mathrm{IIVersion}$ $=$ ES64L-G09RevD.01।State=1-AlHF=-3513.6167474|RMSD=1.969e-09|Dipole=1.9 $921558,-2.8442456,4.8715654$ Quadrupole $=-9.8969745,17.7955698,-7.898595$ $3,-11.2991198,7.173432,-24.6882764 \mid P G=C 01$ [X(C65H69F3N1O4P1)]॥@

Conf3

1\1|GINC-LOGINISPIRTPSSTPSSIdef2SVPIC65H69F3N1O4P1\JHIOEI07-May-201910

॥\#p tpsstpss/def2svp int=ultrafine empiricaldispersion=gd3 scrf=(smd, solvent $=$ ch2cl2, read,externaliteration, dovacuum) ) Ititlel|0,1|P, 0,0.2416 $6,-0.738169,-1.28005 \backslash 0,0,-1.016607,0.223816,-1.816845 \backslash 0,0,1.012822,0.3$ $57843,-0.237022 \backslash 0,0,1.06476,-1.066331,-2.489337 \backslash 0,0,-0.271828,-1.79001$ $5,-0.298764 \backslash \mathrm{C}, 0,-1.144366,2.178335,-0.441213 \backslash \mathrm{C}, 0,-1.737875,1.021229,-0$ $.953116 \backslash \mathrm{C}, 0,-3.103587,0.668386,-0.710872 \mathrm{IC}, 0,-3.853961,1.506031,0.1039$ $37 \mathrm{H}, 0,-4.908292,1.273857,0.287463 \mathrm{IC}, 0,-3.282386,2.64664,0.737823 \mathrm{IC}, 0$ $-1.900509,2.980031,0.49075 \backslash \mathrm{C}, 0,-1.328512,4.066552,1.219881 \backslash \mathrm{H}, 0,-0.2701$ $32,4.305017,1.080939 \backslash \mathrm{C}, 0,-2.088273,4.807195,2.113544 \backslash \mathrm{H}, 0,-1.622683,5.6$ $28337,2.669644 \mathrm{IC}, 0,-3.460728,4.505204,2.32018 \mathrm{H}, 0,-4.049834,5.099196,3$ $.027241 \backslash \mathrm{C}, 0,-4.041524,3.442556,1.647423 \backslash \mathrm{H}, 0,-5.089409,3.176744,1.82432$ $1 \backslash \mathrm{C}, 0,0.242558,2.549433,-0.843087 \backslash \mathrm{C}, 0,1.290614,1.6368,-0.678356 \backslash \mathrm{C}, 0,2$. $663609,1.991897,-0.853644 \backslash \mathrm{C}, 0,2.952694,3.277711,-1.291425 \backslash \mathrm{H}, 0,3.997355$ ,3.584706,-1.406508IC,0,1.924948,4.211028,-1.609575IC,0,0.546313,3.846 $27,-1.396981 \backslash \mathrm{C}, 0,-0.464605,4.780868,-1.775951 \backslash \mathrm{H}, 0,-1.516762,4.504411,-$ $1.661552 \backslash \mathrm{C}, 0,-0.131967,6.021375,-2.300063 \mathrm{H}, 0,-0.926264,6.71728,-2.591$ 894\C, $0,1.228557,6.394026,-2.469873 \backslash \mathrm{H}, 0,1.477887,7.379886,-2.877331 \backslash \mathrm{C}$ $0,2.234562,5.501833,-2.134588 \backslash \mathrm{H}, 0,3.287882,5.768204,-2.278575 \backslash \mathrm{C}, 0,-2.2$ $09259,-0.038367,4.183184 \backslash \mathrm{C}, 0,-1.862898,-1.167958,4.940417 \backslash \mathrm{H}, 0,-2.43151$ $2,-1.426038,5.837513 \backslash \mathrm{C}, 0,-0.785417,-1.96518,4.540201 \backslash \mathrm{H}, 0,-0.526375,-2$. $848623,5.130286 \mid C, 0,-0.072377,-1.657631,3.36041 \backslash \mathrm{C}, 0,-0.423347,-0.50925$ $2,2.613696 \backslash \mathrm{H}, 0,0.139591,-0.231239,1.719441 \backslash \mathrm{C}, 0,-1.481545,0.298315,3.02$ $8443 \mathrm{lH}, 0,-1.743057,1.188243,2.449832 \mathrm{lC}, 0,1.036413,-2.533648,2.925129 \mathrm{IC}$ $, 0,1.895182,-3.204966,3.950623 \mathrm{H}, 0,2.882725,-3.465274,3.540212 \mathrm{H}, 0,1.4$ $09203,-4.137048,4.294214 \backslash \mathrm{H}, 0,2.011309,-2.546965,4.825457 \backslash \mathrm{N}, 0,1.24339$,$2.668649,1.638871 \backslash \mathrm{H}, 0,0.576537,-2.193567,0.919137 \backslash \mathrm{C}, 0,2.232276,-3.4624$ $21,0.976155 \backslash \mathrm{C}, 0,2.947568,-2.85781,-0.07484 \backslash \mathrm{H}, 0,2.80911,-1.7968,-0.3001$ $8 \mathrm{IC}, 0,3.855694,-3.625873,-0.812226 \mathrm{IH}, 0,4.415654,-3.154505,-1.624646 \mathrm{IC}$, $0,4.049619,-4.982883,-0.508245 \backslash \mathrm{C}, 0,3.328463,-5.577558,0.538846 \backslash \mathrm{H}, 0,3.4$ $66584,-6.638529,0.771829 \mathrm{CC}, 0,2.410418,-4.823813,1.282807 \backslash \mathrm{H}, 0,1.814596$, $-5.294328,2.069545 \backslash \mathrm{C}, 0,3.768876,1.040709,-0.499623 \backslash \mathrm{C}, 0,4.530168,0.4081$ $71,-1.51812 \backslash \mathrm{C}, 0,4.099866,0.837777,0.868118 \backslash \mathrm{C}, 0,5.65483,-0.355383,-1.15$ $0667 \backslash \mathrm{C}, 0,5.233004,0.065579,1.183057 \backslash \mathrm{C}, 0,6.040214,-0.518464,0.19143 \backslash \mathrm{H}, 0$

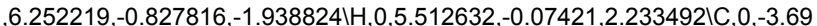
$3176,-0.55245,-1.34817 \backslash \mathrm{C}, 0,-4.09868,-1.656352,-0.549469 \backslash \mathrm{C}, 0,-3.860104$, $-0.602548,-2.762035 \backslash \mathrm{C}, 0,-4.673764,-2.776697,-1.181521 \backslash \mathrm{C}, 0,-4.43362,-1$. $746546,-3.342218 \mid \mathrm{C}, 0,-4.852562,-2.846025,-2.571417 \backslash \mathrm{H}, 0,-4.987947,-3.63$ $0182,-0.56794 \mathrm{H}, 0,-4.566258,-1.775448,-4.430152 \backslash \mathrm{H}, 0,4.761927,-5.577226$ $-1.089955|C, 0,-3.37998,0.830048,4.574186| C, 0,-3.482485,0.570271,-3.66$ $9909 \mathrm{IH}, 0,-3.018831,1.350703,-3.044239 \backslash \mathrm{C}, 0,-3.912421,-1.68865,0.968738 \backslash$ $\mathrm{C}, 0,-5.475252,-4.078883,-3.220764 \mathrm{|H}, 0,-5.748261,-4.770793,-2.401732 \mathrm{C}$, $0,-5.267802,-1.685436,1.704272 \backslash \mathrm{H}, 0,-5.117338,-1.68941,2.798488 \mathrm{H}, 0,-5$. $862972,-0.791687,1.445409 \mathrm{H}, 0,-5.865974,-2.577907,1.442465 \backslash \mathrm{C}, 0,-4.4660$ $96,-4.806443,-4.1333 \mathrm{H}, 0,-4.162333,-4.165031,-4.981126 \backslash \mathrm{H}, 0,-3.554311,-$ $5.087428,-3.576699 \mathrm{H}, 0,-4.911812,-5.72828,-4.55049 \backslash \mathrm{C}, 0,-6.766643,-3.73$ $1412,-3.989471 \backslash \mathrm{H}, 0,-7.237197,-4.646918,-4.392913 \backslash \mathrm{H}, 0,-7.5004,-3.229699$ ,-3.333405।H,0,-6.556493,-3.058811,-4.841412 IC, $0,-2.447878,0.166097,-4$ $.73924 \mathrm{|H}, 0,-2.862276,-0.577963,-5.444534 \mathrm{|H}, 0,-2.141683,1.049948,-5.328$ $962 \mathrm{H}, 0,-1.546587,-0.261603,-4.269369 \mid \mathrm{C}, 0,-4.738967,1.198386,-4.309056$ $\mathrm{IH}, 0,-4.465741,2.082064,-4.914883 \backslash \mathrm{H}, 0,-5.255658,0.480766,-4.972833 \backslash \mathrm{H}, 0$ ,-5.457607,1.523673,-3.53528।C,0,7.298644,-1.295186,0.573135\H,0,7.414 $847,-1.198151,1.669117 \backslash \mathrm{C}, 0,4.164457,0.558984,-2.996249 \mathrm{H}, 0,3.147915,0$. $986535,-3.038982 \backslash \mathrm{C}, 0,3.284257,1.49624,1.983397 \mathrm{VH}, 0,2.262191,1.650344,1$ .597399।C, $0,7.171951,-2.797805,0.252526 \backslash \mathrm{H}, 0,8.082748,-3.339621,0.56764$ $8 \mathrm{H}, 0,6.308413,-3.245335,0.773821 \backslash \mathrm{H}, 0,7.038323,-2.965336,-0.831826 \backslash \mathrm{C}, 0$ ,8.557426,-0.693443,-0.084072/H,0,8.519168,-0.787057,-1.184998/H, $0,8.6$ $64078,0.378417,0.161568 \backslash \mathrm{H}, 0,9.465797,-1.217913,0.265085 \backslash \mathrm{C}, 0,3.159442,0$ $.620502,3.2435 \mathrm{H}, 0,2.833078,-0.401716,2.986772 \mathrm{H}, 0,4.115609,0.542922,3$ $.792208 \mathrm{lH}, 0,2.41657,1.054577,3.93625 \backslash \mathrm{C}, 0,3.856077,2.887034,2.332166 \mathrm{IH}$, $0,3.883398,3.541888,1.443427 \backslash \mathrm{H}, 0,3.236295,3.381074,3.10303 \backslash \mathrm{H}, 0,4.88675$ $1,2.799263,2.72298 \backslash \mathrm{C}, 0,5.124822,1.53447,-3.707946 \mathrm{VH}, 0,5.127716,2.52538$ $6,-3.221196 \mathrm{H}, 0,6.161215,1.148277,-3.694122 \backslash \mathrm{H}, 0,4.826982,1.672945,-4.7$ $63679 \mathrm{lC}, 0,4.108728,-0.795852,-3.727838 \mathrm{H}, 0,3.787281,-0.644667,-4.77477$ $3 \mathrm{H}, 0,5.095942,-1.292607,-3.754728 \mathrm{H}, 0,3.37732,-1.463515,-3.245132 \mathrm{lC}, 0$ ,-3.039505,-2.881729,1.40903\H,0,-3.539636,-3.847811,1.210894\H,0,-2.0 $75469,-2.868726,0.874425 \mathrm{H}, 0,-2.839319,-2.825394,2.494575 \mathrm{lH}, 0,-3.37452$ $5,-0.772929,1.26227 \mathrm{~F}, 0,-3.989234,0.413059,5.708342 \mathrm{~F}, 0,-4.331515,0.84$ 8725,3.597733IF,0,-3.007782,2.122007,4.770558IIVersion=ES64L-G09RevD.0 
1IState=1-AlHF=-3513.6166304IRMSD=1.907e-09|Dipole=2.1122496,-2.446963 7,5.0205169lQuadrupole=-9.3047597,13.4565063,-4.1517467,-11.6322936,8. 7764639,-26.6297609IPG=C01 [X(C65H69F3N1O4P1)]川@

\section{TRIP/2E Type II}

Confo

1।1IGINC-WORKER0ISPIRTPSSTPSSIdef2SVPIC65H69F3N1O4P1\JHIOEI05-May-2019

$10 \backslash ॥ p$ tpsstpss/def2svp int=ultrafine empiricaldispersion=gd3 scrf=(sm $\mathrm{d}$, solvent $=\mathrm{ch} 2 \mathrm{cl} 2$, read, externaliteration, dovacuum) \titlell0,1\P, $0,-0.2$ $51952,-0.545172,-0.840162 \backslash 0,0,-1.686442,0.133539,-1.371612 \backslash \mathrm{O}, 0,0.39618$ $4,0.802852,-0.02757 \backslash 0,0,0.524169,-0.918084,-2.066325 \backslash 0,0,-0.512652,-1$ $506699,0.316919 \mid \mathrm{C}, 0,-2.083801,2.125352,-0.109826 \backslash \mathrm{C}, 0,-2.467103,0.83149$ $8,-0.471335 \backslash C, 0,-3.670434,0.210293,-0.013452 \backslash C, 0,-4.472848,0.927311,0$. $863281 \backslash \mathrm{H}, 0,-5.416291,0.492632,1.208529 \backslash \mathrm{C}, 0,-4.091737,2.21078,1.350367 \backslash$ C, $0,-2.872426,2.819863,0.877657 \backslash \mathrm{C}, 0,-2.472397,4.063822,1.453238 \backslash \mathrm{H}, 0,-1$ $.52827,4.51782,1.139665 \backslash \mathrm{C}, 0,-3.249096,4.689401,2.41799 \mathrm{H}, 0,-2.913883,5$ $.637663,2.852269 \mid \mathrm{C}, 0,-4.469538,4.106599,2.853337 \mathrm{H}, 0,-5.076122,4.61328$ $2,3.611815 \backslash \mathrm{C}, 0,-4.877781,2.889836,2.329695 \backslash \mathrm{H}, 0,-5.804779,2.417418,2.67$ 4142\C, $0,-0.859525,2.727994,-0.711635 \backslash \mathrm{C}, 0,0.361696,2.0518,-0.617078 \mathrm{IC}$ $0,1.606158,2.633806,-1.014384 \backslash \mathrm{C}, 0,1.578032,3.891831,-1.600916 \mathrm{H}, 0,2.51$ $7839,4.368235,-1.899021 \backslash \mathrm{C}, 0,0.353933,4.581169,-1.83898 \backslash \mathrm{C}, 0,-0.88807,3$ $997164,-1.394972 \backslash \mathrm{C}, 0,-2.101982,4.692299,-1.682225 \mathrm{H}, 0,-3.055235,4.2448$ $16,-1.386108 \mathrm{IC}, 0,-2.088941,5.912479,-2.341871 \mathrm{WH}, 0,-3.033926,6.423533,-$ $2.556818 \mathrm{IC}, 0,-0.861701,6.501512,-2.748755 \backslash \mathrm{H}, 0,-0.86486,7.468094,-3.264$ 226।C, $0,0.333522,5.843784,-2.504671 \backslash \mathrm{H}, 0,1.28588,6.279069,-2.828444 \backslash \mathrm{C}, 0$ $4.070366,-3.376393,-0.249454 \backslash \mathrm{C}, 0,4.0378,-4.088876,0.964138 \backslash \mathrm{H}, 0,4.6162$ $57,-5.010441,1.077557 \backslash \mathrm{C}, 0,3.265427,-3.613435,2.026168 \mathrm{H}, 0,3.233772,-4$. $174672,2.964424 \backslash \mathrm{C}, 0,2.507507,-2.42816,1.877132 \backslash \mathrm{C}, 0,2.566129,-1.715677$, $0.661156 \mathrm{IH}, 0,2.064251,-0.751068,0.561529 \mathrm{IC}, 0,3.334336,-2.193702,-0.402$ $201 \mathrm{lH}, 0,3.367777,-1.631715,-1.335922 \mathrm{C}, 0,1.695372,-1.908959,2.998581 \mathrm{lC}$ $, 0,2.237219,-1.99344,4.390535 \mathrm{H}, 0,1.821551,-1.205811,5.037672 \backslash \mathrm{H}, 0,3.33$

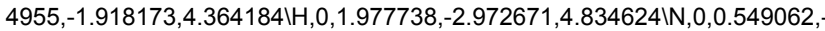
$1.343593,2.70497 \backslash \mathrm{H}, 0,0.19816,-1.372517,1.671547 \backslash \mathrm{C}, 0,-0.376241,-0.69317$ $6,3.577985 \backslash \mathrm{C}, 0,-0.934058,0.513627,3.11298 \mathrm{H}, 0,-0.630648,0.907413,2.138$ $692 \mathrm{IC}, 0,-1.864264,1.190537,3.910151 \mathrm{VH}, 0,-2.285127,2.136889,3.558827 \mathrm{C}$, $0,-2.261834,0.653957,5.145178 \mathrm{CC}, 0,-1.72626,-0.566991,5.584764 \mathrm{IH}, 0,-2.0$ $51796,-1.001034,6.53581 \backslash \mathrm{C}, 0,-0.780856,-1.248603,4.806012 \backslash \mathrm{H}, 0,-0.394026$ ,-2.216991,5.132053\C,0,2.896947,1.922099,-0.739085\C,0,3.631145,1.318 $277,-1.792018 \mid \mathrm{C}, 0,3.390329,1.872093,0.596059 \mathrm{IC}, 0,4.855227,0.689278,-1$. $488904 \mathrm{IC}, 0,4.61569,1.231304,0.845078 \mathrm{IC}, 0,5.363592,0.627377,-0.182537 \backslash \mathrm{H}$ $, 0,5.422647,0.210529,-2.296117 \mathrm{VH}, 0,4.995691,1.195708,1.870811 \mathrm{lC}, 0,-4.0$ $58719,-1.150209,-0.508425 \backslash C, 0,-4.026703,-2.271901,0.364245 \backslash C, 0,-4.4792$ $54,-1.313049,-1.858056 \backslash C, 0,-4.465015,-3.51933,-0.121424 \backslash \mathrm{C}, 0,-4.89411$,$2.582789,-2.296022 \backslash \mathrm{C}, 0,-4.903596,-3.700043,-1.44274 \mathrm{|H}, 0,-4.45097,-4.38$ $7164,0.548438 \mathrm{H}, 0,-5.219111,-2.70154,-3.336389 \mathrm{VH}, 0,-2.999244,1.182388$, $5.758386 \mid \mathrm{C}, 0,4.995207,-3.828013,-1.351502 \mathrm{IC}, 0,-4.505466,-0.147924,-2.8$ $49377 \backslash \mathrm{H}, 0,-4.148374,0.75401,-2.3246 \backslash \mathrm{C}, 0,-3.516566,-2.168645,1.803758 \mathrm{IC}$ $, 0,-5.349586,-5.072379,-1.93917 \backslash \mathrm{H}, 0,-5.301435,-5.760815,-1.074433 \backslash \mathrm{C}, 0$ $-4.679989,-2.130262,2.816915 \mathrm{H}, 0,-4.293638,-2.020162,3.846978 \mathrm{H}, 0,-5.3$ $65704,-1.288452,2.619205 \mathrm{H}, 0,-5.270276,-3.064698,2.772086 \mathrm{IC}, 0,-4.39281$ $5,-5.613453,-3.022 \backslash \mathrm{H}, 0,-4.409526,-4.973593,-3.92363 \backslash \mathrm{H}, 0,-3.352561,-5.6$ $49988,-2.652098 \mathrm{H}, 0,-4.686039,-6.634725,-3.3277 \backslash \mathrm{C}, 0,-6.806377,-5.05551$ $4,-2.444013 \mathrm{H}, 0,-7.126749,-6.070352,-2.743539 \mathrm{H}, 0,-7.496729,-4.694917$, $-1.660387 \backslash \mathrm{H}, 0,-6.916907,-4.395413,-3.324098 \mathrm{CC}, 0,-3.550195,-0.396414,-4$ $.035022 \mathrm{H}, 0,-3.875478,-1.262501,-4.640774 \mathrm{MH}, 0,-3.524507,0.486835,-4.69$ $987 \backslash \mathrm{H}, 0,-2.525095,-0.587201,-3.675514 \backslash \mathrm{C}, 0,-5.939347,0.151845,-3.332855$ $\mathrm{IH}, 0,-5.944334,1.026589,-4.009004 \backslash \mathrm{H}, 0,-6.366957,-0.703937,-3.887066 \mathrm{H}$, $0,-6.60888,0.374066,-2.482419 \backslash \mathrm{C}, 0,6.690886,-0.072664,0.097563 \backslash \mathrm{H}, 0,6.90$ $5791,-0.709395,-0.780595 \backslash \mathrm{C}, 0,3.138801,1.34194,-3.24045 \backslash \mathrm{H}, 0,2.090887,1$. $688379,-3.228374 \mid \mathrm{C}, 0,2.628759,2.547519,1.739311 \mathrm{IH}, 0,1.556707,2.527806$, $1.479409 \backslash \mathrm{C}, 0,7.836441,0.953886,0.2257 \backslash \mathrm{H}, 0,8.804933,0.444331,0.384216 \backslash \mathrm{H}$ $, 0,7.92009,1.573873,-0.68473 \mathrm{lH}, 0,7.662188,1.63068,1.082848 \backslash \mathrm{C}, 0,6.63040$ $6,-0.991765,1.332753 \mathrm{H}, 0,6.511514,-0.411484,2.266048 \mathrm{H}, 0,5.788852,-1.7$ $01747,1.263008 \backslash \mathrm{H}, 0,7.563887,-1.57615,1.422711 \backslash \mathrm{C}, 0,2.775196,1.824095,3$. $089284 \mathrm{H}, 0,2.550374,0.748668,2.992472 \mathrm{H}, 0,3.794658,1.921889,3.504622 \mathrm{H}$ $, 0,2.076217,2.254437,3.828207 \backslash \mathrm{C}, 0,3.042245,4.030214,1.862013 \mathrm{MH}, 0,2.876$ $749,4.570615,0.913377 \mathrm{VH}, 0,2.455801,4.535125,2.651788 \mathrm{H}, 0,4.113753,4.11$ $6506,2.120907 \backslash \mathrm{C}, 0,3.960282,2.348706,-4.073364 \mathrm{H}, 0,3.916668,3.362742,-3$ $.637623 \backslash \mathrm{H}, 0,5.023164,2.046532,-4.120566 \mathrm{IH}, 0,3.576208,2.40442,-5.108578$ IC, $0,3.140843,-0.056214,-3.88981 \backslash \mathrm{H}, 0,2.760996,0.00966,-4.926259 \mathrm{H}, 0,4$. $155696,-0.491277,-3.937157 \backslash \mathrm{H}, 0,2.480238,-0.73615,-3.328034 \backslash \mathrm{C}, 0,-2.5335$ $52,-3.303975,2.153525 \mathrm{lH}, 0,-3.035274,-4.288621,2.176648 \mathrm{H}, 0,-1.708758,-$ $3.34065,1.423742 \backslash \mathrm{H}, 0,-2.10222,-3.133213,3.155677 \mathrm{VH}, 0,-2.959905,-1.2199$ $7,1.886103 \mathrm{IF}, 0,6.283339,-3.447421,-1.101036 \mathrm{IF}, 0,5.01629,-5.177441,-1.4$ 8729IF $, 0,4.665221,-3.304462,-2.556418$ IIVersion=ES64L-G09RevD.01।State= 
1-AlHF=-3513.6179709|RMSD =2.124e-09IDipole $=0.8325238,-0.1111922,6.2971$ 94IQuadrupole=-12.1824579,-1.5461594,13.7286173,-8.0052176,9.0546708,21.7894102\PG=C01 [X(C65H69F3N1O4P1)]川@

Conf1

1\1\GINC-WORKER1ISPIRTPSSTPSSIdef2SVPIC65H69F3N1O4P1\JHIOE\29-Apr-2019

IO\} \# p \text { tpsstpss/def2svp int=ultrafine empiricaldispersion=gd3 scrf } = ( \mathrm { sm }

$\mathrm{d}$, solvent=ch2cl2, read,externaliteration, dovacuum) $\mid$ titile $\| \mathbf{0}, 1 \backslash \mathrm{P}, 0,-0.2$

48114,-0.485061,-0.9228।O,0,-1.651579,0.261392,-1.44765।O,0,0.419767,0

$.796826,-0.023022 \backslash 0,0,0.545179,-0.815673,-2.150073 \backslash 0,0,-0.562253,-1.49$ $8432,0.175229 \backslash \mathrm{C}, 0,-2.025525,2.184446,-0.077923 \backslash \mathrm{C}, 0,-2.433915,0.92503,-$ $0.522562 \backslash \mathrm{C}, 0,-3.661407,0.310096,-0.124352 \backslash \mathrm{C}, 0,-4.459601,0.99269,0.7830$ $82 \backslash \mathrm{H}, 0,-5.419562,0.562791,1.08644 \backslash \mathrm{C}, 0,-4.055121,2.234104,1.353276 \mid \mathrm{C}, 0$, $-2.814045,2.839889,0.936139 \backslash \mathrm{C}, 0,-2.394694,4.037855,1.590129 \backslash \mathrm{H}, 0,-1.435$ $448,4.487016,1.318072 \mathrm{IC}, 0,-3.172288,4.623771,2.578893 \mathrm{H}, 0,-2.822402,5$. $536228,3.074023 \mathrm{IC}, 0,-4.412957,4.046079,2.960605 \mathrm{IH}, 0,-5.019915,4.521784$ ,3.738567\C,0,-4.840967,2.872825,2.35934\H,0,-5.784435,2.403772,2.6607 $25 \backslash \mathrm{C}, 0,-0.774106,2.78886,-0.618733 \backslash \mathrm{C}, 0,0.429152,2.080385,-0.532645 \backslash \mathrm{C}, 0$ $1.696649,2.660497,-0.852918 \backslash \mathrm{C}, 0,1.711256,3.952277,-1.362034 \backslash \mathrm{H}, 0,2.669$ $695,4.425915,-1.599907 \backslash \mathrm{C}, 0,0.508527,4.678543,-1.599224 \backslash \mathrm{C}, 0,-0.75761,4$. $093893,-1.230883 \backslash \mathrm{C}, 0,-1.949393,4.826423,-1.517463 \mathrm{H}, 0,-2.919319,4.3811$ $84,-1.277 \backslash \mathrm{C}, 0,-1.893671,6.079358,-2.110258 \backslash \mathrm{H}, 0,-2.822193,6.618326,-2.3$ $28605 \backslash \mathrm{C}, 0,-0.643564,6.666949,-2.443037 \backslash \mathrm{H}, 0,-0.613372,7.659375,-2.90587$ $5 \mid \mathrm{C}, 0,0.531856,5.975579,-2.195271 \mathrm{WH}, 0,1.501803,6.410474,-2.46244 \backslash \mathrm{C}, 0,3$ $.985104,-3.40304,-0.417265 \backslash \mathrm{C}, 0,3.896421,-4.214082,0.729855 \mathrm{IH}, 0,4.44551$ $-5.158843,0.780385 \backslash \mathrm{C}, 0,3.106534,-3.806527,1.807553 \backslash \mathrm{H}, 0,3.031344,-4.44$ $3031,2.693917 \backslash \mathrm{C}, 0,2.386864,-2.590983,1.738594 \backslash \mathrm{C}, 0,2.500526,-1.780966,0$ $.589391 \mathrm{~W}, 0,2.026158,-0.798113,0.557683 \mathrm{IC}, 0,3.287073,-2.189765,-0.4888$ $85 \backslash \mathrm{H}, 0,3.365246,-1.550558,-1.368737 \backslash \mathrm{C}, 0,1.55629,-2.139436,2.875442 \backslash \mathrm{C}, 0$ ,2.049474,-2.351017,4.271855\H,0,1.644612,-1.597523,4.965121\H,0,3.149 $617,-2.322065,4.285122 \mathrm{H}, 0,1.734218,-3.348973,4.630671 \mathrm{WN}, 0,0.435649,-1$ $.519203,2.593064 \mathrm{IH}, 0,0.113746,-1.466883,1.550802 \mathrm{IC}, 0,-0.493085,-0.8971$ $48,3.482715 \backslash \mathrm{C}, 0,-0.991991,0.358884,3.085757 \backslash \mathrm{H}, 0,-0.64619,0.804502,2.14$ $8485 \backslash \mathrm{C}, 0,-1.919143,1.017159,3.901714 \backslash \mathrm{H}, 0,-2.293734,2.000804,3.604371 \backslash \mathrm{C}$ $, 0,-2.372325,0.414992,5.086491 \backslash \mathrm{C}, 0,-1.895666,-0.852607,5.456235 \backslash \mathrm{H}, 0,-2$ $265082,-1.336227,6.366437 \backslash \mathrm{C}, 0,-0.953764,-1.517397,4.658702 \backslash \mathrm{H}, 0,-0.612$ $101,-2.519509,4.928719 \mid \mathrm{C}, 0,2.965433,1.910583,-0.57768 \mathrm{IC}, 0,3.739383,1.3$ $76229,-1.639282 \backslash C, 0,3.403227,1.76147,0.77036 \backslash C, 0,4.943534,0.710932,-1$ $331527 \backslash \mathrm{C}, 0,4.609845,1.087932,1.023082 \backslash \mathrm{C}, 0,5.394456,0.547925,-0.013259 \backslash$ $\mathrm{H}, 0,5.539806,0.283482,-2.146868 \mathrm{H}, 0,4.946706,0.975539,2.058304 \backslash \mathrm{C}, 0,-4$ $080668,-1.002127,-0.715232 \backslash \mathrm{C}, 0,-4.087463,-2.183884,0.077835 \mid C, 0,-4.493$ $733,-1.057077,-2.073525 \backslash C, 0,-4.553928,-3.380853,-0.494781 \backslash C, 0,-4.93935$ $4,-2.283559,-2.601811 \backslash \mathrm{C}, 0,-4.986494,-3.455557,-1.830772 \backslash \mathrm{H}, 0,-4.56617,-$ $4.28862,0.118803 \backslash \mathrm{H}, 0,-5.261689,-2.327886,-3.649495 \backslash \mathrm{H}, 0,-3.106917,0.929$ $696,5.714591 \backslash \mathrm{C}, 0,4.931229,-3.781802,-1.528988 \backslash \mathrm{C}, 0,-4.482382,0.176679$,$2.97775 \backslash \mathrm{H}, 0,-4.111915,1.029402,-2.384281 \backslash \mathrm{C}, 0,-3.592384,-2.191387,1.526$ $215 \mathrm{IC}, 0,-5.473762,-4.770546,-2.432735 \mathrm{IH}, 0,-5.761376,-4.559934,-3.48009$ 3।C $0,-4.768568,-2.17539,2.524911 \backslash \mathrm{H}, 0,-4.395003,-2.144142,3.564942 \mathrm{VH}, 0$ $,-5.421469,-1.299075,2.37148 \mathrm{H}, 0,-5.390106,-3.083459,2.412479 \mathrm{CC}, 0,-6.7$ $23123,-5.304566,-1.702821 \backslash \mathrm{H}, 0,-6.495502,-5.552004,-0.649458 \mathrm{H}, 0,-7.537$ $219,-4.557732,-1.707789 \backslash \mathrm{H}, 0,-7.096609,-6.223527,-2.190873 \backslash \mathrm{C}, 0,-4.35162$ $2,-5.828612,-2.459822 \mathrm{H}, 0,-4.698064,-6.751857,-2.959759) \mathrm{H}, 0,-3.465104$, $-5.454652,-3.002363 \mathrm{|H}, 0,-4.033623,-6.098661,-1.435899 \mathrm{IC}, 0,-3.517405,-0$ $.00556,-4.167376 \backslash \mathrm{H}, 0,-3.85454,-0.815539,-4.840456 \backslash \mathrm{H}, 0,-3.461505,0.9242$ $7,-4.763223 \mathrm{H}, 0,-2.502005,-0.24753,-3.810772 \backslash \mathrm{C}, 0,-5.90322,0.543866,-3$. 45363\H $, 0,-5.880854,1.468561,-4.05924 \backslash \mathrm{H}, 0,-6.341202,-0.256719,-4.07772$ $3 \mathrm{H}, 0,-6.579043,0.714161,-2.596224 \backslash \mathrm{C}, 0,6.698298,-0.192356,0.273305 \backslash \mathrm{H}, 0$ ,6.960801,-0.743449,-0.648853\C,0,3.307154,1.498294,-3.102041\H,0,2.27 $97,1.900996,-3.112625 \backslash \mathrm{C}, 0,2.60542,2.371917,1.925987 \backslash \mathrm{H}, 0,1.542214,2.370$ $606,1.631673 \backslash \mathrm{C}, 0,7.841893,0.800627,0.570144 \mathrm{IH}, 0,8.795517,0.264318,0.73$ $0299 \mathrm{H}, 0,7.982474,1.50816,-0.266514 \mathrm{IH}, 0,7.624878,1.389694,1.480666 \mathrm{IC}, 0$ $, 6.554523,-1.227113,1.406135 \mathrm{H}, 0,6.379981,-0.739057,2.382543 \mathrm{H}, 0,5.714$ $47,-1.915512,1.213516 \mathrm{IH}, 0,7.476555,-1.829411,1.49696 \mathrm{IC}, 0,2.707288,1.57$ $4262,3.237891 \mathrm{lH}, 0,2.481695,0.506924,3.075749 \mathrm{H}, 0,3.713567,1.64448,3.68$ $9217 \mathrm{\backslash H}, 0,1.987189,1.967118,3.977329 \mathrm{IC}, 0,3.017161,3.844113,2.145166 \mathrm{lH}, 0$ ,2.881531,4.437246,1.223917\H,0,2.406818,4.306062,2.943029\H,0,4.08017 $3,3.911813,2.441605 \backslash \mathrm{C}, 0,4.210558,2.495863,-3.85651 \backslash \mathrm{H}, 0,4.209748,3.4870$ $94,-3.368973 \backslash \mathrm{H}, 0,5.255926,2.136577,-3.89097 \backslash \mathrm{H}, 0,3.861769,2.625711,-4.8$ $97565 \backslash \mathrm{C}, 0,3.259251,0.131301,-3.814024 \mathrm{H}, 0,2.945072,0.264475,-4.865916 \backslash$ $\mathrm{H}, 0,4.245876,-0.366475,-3.823343 \mathrm{H}, 0,2.526193,-0.527782,-3.32076 \backslash \mathrm{C}, 0,-$ $2.655,-3.381299,1.814481 \backslash \mathrm{H}, 0,-3.193498,-4.346076,1.778996 \mathrm{H}, 0,-1.82738$ $8,-3.407692,1.087477 \backslash \mathrm{H}, 0,-2.22333,-3.284523,2.826397 \backslash \mathrm{H}, 0,-3.003684,-1$ $270617,1.675258 \mathrm{IF}, 0,6.213574,-3.416311,-1.23059 \mathrm{IF}, 0,4.958105,-5.118841$ $,-1.753577 \backslash \mathrm{F}, 0,4.621834,-3.179848,-2.702842$ IIVersion=ES64L-G09RevD.01। State $=1-A l H F=-3513.617892 \backslash R M S D=2.134 \mathrm{e}-09$ IDipole $=0.6465577,-0.5445866,6$ $.321251 \backslash$ Quadrupole $=-12.7337149,2.208718,10.5249969,-7.6431537,7.197817$ 8,-22.9913322।PG=C01 [X(C65H69F3N1O4P1)]॥@ 


\section{Conf2}

1।1।GINC-WORKEROISPIRTPSSTPSSIdef2SVPIC65H69F3N1O4P1\JHIOEI05-May-2019

IO|॥p tpsstpss/def2svp int=ultrafine empiricaldispersion=gd3 scrf $=(\mathrm{sm}$

$\mathrm{d}$, solvent $=$ ch2cl2, read,externaliteration, dovacuum) \titlell0,1 $1 \mathrm{P}, 0,-0.1$

$99774,-0.548596,-0.802434 \backslash 0,0,-1.605893,0.13503,-1.40288810,0,0.431112$

$, 0.805965,0.01125910,0,0.62044,-0.954142,-1.988707 \backslash 0,0,-0.522552,-1.48$ $4675,0.360049 \mid \mathrm{C}, 0,-2.027101,2.15611,-0.197761 \backslash \mathrm{C}, 0,-2.411105,0.858798$,$0.545394 \backslash \mathrm{C}, 0,-3.636843,0.260608,-0.116802 \backslash \mathrm{C}, 0,-4.463175,1.006342,0.712$ $521 \backslash \mathrm{H}, 0,-5.42443,0.590675,1.031037 \backslash \mathrm{C}, 0,-4.086752,2.297219,1.183457 \backslash \mathrm{C}, 0$ ,-2.843769,2.882003,0.743436\C,0,-2.452127,4.135441,1.304237\H,0,-1.49 $1351,4.571713,1.017484 \backslash \mathrm{C}, 0,-3.258919,4.793118,2.221846 \mathrm{H}, 0,-2.93031,5$. $748574,2.645263 \mathrm{IC}, 0,-4.501808,4.233882,2.623257 \mathrm{MH}, 0,-5.132121,4.765617$ ,3.344327\C, $0,-4.90231,3.008149,2.114746 \backslash \mathrm{H}, 0,-5.84705,2.553776,2.43436$ $6|\mathrm{C}, 0,-0.772755,2.731555,-0.761896 \backslash \mathrm{C}, 0,0.435607,2.044438,-0.600159| \mathrm{C}, 0$ $1.701992,2.608832,-0.947496 \mid \mathrm{C}, 0,1.713564,3.859488,-1.551281 \mathrm{lH}, 0,2.671$ $206,4.323751,-1.809351 \backslash \mathrm{C}, 0,0.509822,4.558071,-1.854282 \backslash \mathrm{C}, 0,-0.757255,3$ $.989934,-1.46476 \mathrm{IC}, 0,-1.94934,4.692463,-1.817872 \backslash \mathrm{H}, 0,-2.91994,4.255771$ $,-1.565246 \backslash \mathrm{C}, 0,-1.892975,5.906151,-2.487 \backslash \mathrm{H}, 0,-2.821743,6.423193,-2.752$ $274 \backslash \mathrm{C}, 0,-0.641881,6.481315,-2.837595 \backslash \mathrm{H}, 0,-0.611013,7.443506,-3.360359 \backslash$ C, $0,0.533802,5.814438,-2.531622 \backslash \mathrm{H}, 0,1.505039,6.237642,-2.812478 \mid \mathrm{C}, 0,3$ $87503,-3.512219,-0.038626 \backslash \mathrm{C}, 0,3.695489,-4.262052,1.139613 \backslash \mathrm{H}, 0,4.158076$ ,-5.248537,1.241368\C,0,2.927122,-3.740854,2.182368\H,0,2.781766,-4.32 $6622,3.094751 \backslash \mathrm{C}, 0,2.311627,-2.475475,2.042688 \backslash \mathrm{C}, 0,2.517391,-1.726477,0$ $.865627 \backslash \mathrm{H}, 0,2.115327,-0.714428,0.777278 \mathrm{CC}, 0,3.294409,-2.244246,-0.1737$ $83 \mathrm{IH}, 0,3.453249,-1.650442,-1.074817 \backslash \mathrm{C}, 0,1.488218,-1.917218,3.137643 \mathrm{IC}$ $0,1.963929,-2.051658,4.548967 \backslash \mathrm{H}, 0,1.586481,-1.236289,5.185514 \mathrm{IH}, 0,3.06$ $4184,-2.066009,4.568466 \mathrm{H}, 0,1.607829,-3.009145,4.973465 \mathrm{WN}, 0,0.391474,-$ $1.284602,2.798018 \mathrm{lH}, 0,0.090403,-1.308785,1.747074 \mathrm{IC}, 0,-0.53786,-0.5855$ $64,3.626598 \mathrm{IC}, 0,-1.036617,0.630109,3.118338 \mathrm{H}, 0,-0.68868,0.991709,2.14$ 636 IC $, 0,-1.967352,1.357275,3.869186 \mathrm{IH}, 0,-2.342822,2.309533,3.484086 \mathrm{IC}$ $0,-2.424779,0.863081,5.101222 \mathrm{CC}, 0,-1.948781,-0.366165,5.583538 \mathrm{H}, 0,-2$. $321046,-0.767966,6.531576 \backslash \mathrm{C}, 0,-1.00387,-1.098395,4.85169 \mathrm{VH}, 0,-0.666811$ $,-2.07278,5.212209 \mid \mathrm{C}, 0,2.980165,1.905149,-0.601172 \backslash \mathrm{C}, 0,3.778714,1.3135$ $01,-1.615481|C, 0,3.418151,1.888542,0.752563| C, 0,5.023192,0.757885,-1.2$ $62063 \backslash \mathrm{C}, 0,4.669061,1.319426,1.05314 \backslash \mathrm{C}, 0,5.495639,0.763498,0.061066 \backslash \mathrm{H}, 0$ $, 5.641631,0.308567,-2.047655 \mathrm{lH}, 0,5.023486,1.318206,2.090105 \mathrm{IC}, 0,-4.025$ $412,-1.105413,-0.596509 \backslash \mathrm{C}, 0,-4.021341,-2.212598,0.294787 \backslash \mathrm{C}, 0,-4.420427$ ,-1.286242,-1.951283\C,0,-4.461335,-3.463763,-0.179444\C,0,-4.83914,-2 $.558962,-2.377006 \mathrm{IC}, 0,-4.876331,-3.66185,-1.506017 \backslash \mathrm{H}, 0,-4.46942,-4.320$ $213,0.5049 \mathrm{H}, 0,-5.144982,-2.691616,-3.421512 \backslash \mathrm{H}, 0,-3.162697,1.430603,5$. $677757 \backslash \mathrm{C}, 0,4.735202,-4.083088,-1.13898 \mathrm{IC}, 0,-4.414298,-0.136095,-2.9598$ $47 \mathrm{VH}, 0,-4.05668,0.76905,-2.441017 \backslash \mathrm{C}, 0,-3.543502,-2.087929,1.74326 \backslash \mathrm{C}, 0$, $-5.326651,-5.037868,-1.987987 \backslash \mathrm{H}, 0,-5.298114,-5.712977,-1.111961 \backslash \mathrm{C}, 0,-4$ $.730216,-2.00278,2.726392 \mathrm{H}, 0,-4.366996,-1.878576,3.763232 \mathrm{H}, 0,-5.3926$ $74,-1.151323,2.494311 \mathrm{lH}, 0,-5.339328,-2.925275,2.686396 \mathrm{lC}, 0,-4.358385,-$ $5.605062,-3.04683 \mathrm{lH}, 0,-4.356368,-4.981138,-3.959729 \mathrm{lH}, 0,-3.324138,-5.6$ $43394,-2.66066 \mathrm{HH}, 0,-4.655485,-6.629215,-3.338892 \mathrm{lC}, 0,-6.775281,-5.0160$ $74,-2.515628 \mathrm{H}, 0,-7.100395,-6.032768,-2.803514 \mathrm{MH}, 0,-7.474326,-4.636262$ ,-1.748952।H,0,-6.865952,-4.369662,-3.408096।C,0,-3.438757,-0.411768,4.122684\H,0,-3.760383,-1.283698,-4.721965\H,0,-3.391078,0.461278,-4.7 $99659 \mathrm{H}, 0,-2.422901,-0.607425,-3.739706 \backslash \mathrm{C}, 0,-5.835763,0.172441,-3.4729$ $5 \mathrm{\backslash H}, 0,-5.819343,1.037,-4.16188 \backslash \mathrm{H}, 0,-6.263477,-0.686643,-4.021992 \backslash \mathrm{H}, 0$,$6.51661,0.41391,-2.636785 \backslash \mathrm{C}, 0,6.864018,0.186577,0.416452 \backslash \mathrm{H}, 0,7.012486$, $0.351193,1.500425 \mathrm{IC}, 0,3.334578,1.284179,-3.079673 \mathrm{IH}, 0,2.28426,1.621223$ $,-3.114174 \backslash \mathrm{C}, 0,2.585453,2.541878,1.858711 \backslash \mathrm{H}, 0,1.529176,2.50719,1.54318$ $5 \backslash \mathrm{C}, 0,6.925975,-1.332279,0.158923 \mathrm{H}, 0,7.913447,-1.738312,0.445766 \mathrm{lH}, 0$, $6.155496,-1.867452,0.739196 \mathrm{H}, 0,6.764407,-1.56155,-0.909159 \backslash \mathrm{C}, 0,7.9978$ $38,0.920898,-0.328222 \backslash \mathrm{H}, 0,7.924165,0.765866,-1.420391 \backslash \mathrm{H}, 0,7.964537,2.0$ $08406,-0.13649 \mathrm{HH}, 0,8.984891,0.545072,-0.001759 \mathrm{lC}, 0,2.672991,1.808471,3$ $.209007 \backslash \mathrm{H}, 0,2.462963,0.731686,3.093726 \mathrm{H}, 0,3.669461,1.912895,3.675215 \backslash$ $\mathrm{H}, 0,1.934442,2.226315,3.916034 \backslash \mathrm{C}, 0,2.967832,4.02992,2.013394 \backslash \mathrm{H}, 0,2.843$ $057,4.574286,1.060714 \backslash \mathrm{H}, 0,2.332498,4.519715,2.774389 \backslash \mathrm{H}, 0,4.022654,4.13$ $162,2.328864 \mathrm{IC}, 0,4.173095,2.266179,-3.924492 \mathrm{H}, 0,4.112469,3.294314,-3$. $525122 \backslash \mathrm{H}, 0,5.238633,1.969945,-3.934325 \backslash \mathrm{H}, 0,3.815896,2.283561,-4.970579$ IC, $0,3.36873,-0.138461,-3.673478 \mid \mathrm{H}, 0,3.038577,-0.114722,-4.728633 \backslash \mathrm{H}, 0$, $4.38562,-0.570789,-3.653394 \backslash \mathrm{H}, 0,2.682607,-0.798294,-3.118255 \backslash \mathrm{C}, 0,-2.59$ $4175,-3.235098,2.143013 \backslash \mathrm{H}, 0,-3.11834,-4.207582,2.180857 \backslash \mathrm{H}, 0,-1.754612$, $-3.309334,1.433285 \backslash \mathrm{H}, 0,-2.181037,-3.047375,3.149796 \backslash \mathrm{H}, 0,-2.968789,-1.1$ $49248,1.816951 \backslash F, 0,5.983736,-4.390295,-0.689173 \backslash F, 0,4.218331,-5.235774$ ,-1.640848IF,0,4.889151,-3.230379,-2.181083IIVersion=ES64L-G09RevD.01। State $=1-A l H F=-3513.6172209 \mid R M S D=2.115 e-09 \backslash$ Dipole $=0.5290941,0.0499115,6$ $.3688697 \backslash$ Quadrupole $=-12.6365377,-2.7823625,15.4189002,-5.5071238,7.211$ $5688,-22.5331701 \backslash \mathrm{PG}=\mathrm{C} 01[\mathrm{X}(\mathrm{C} 65 \mathrm{H} 69 \mathrm{~F} 3 \mathrm{~N} 1 \mathrm{O} 4 \mathrm{P} 1)] 1 @$

Conf3

1\1|GINC-WORKER1ISPIRTPSSTPSSIdef2SVPIC65H69F3N1O4P1\JHIOEI05-May-2019 
IO\\p tpsstpss/def2svp int=ultrafine empiricaldispersion=gd3 scrf=(sm $\mathrm{d}$, solvent $=$ ch2cl2, read,externaliteration, dovacuum) \titlell0,1 $1 \mathrm{P}, 0,-0.1$ $80576,-0.486323,-0.86019910,0,-1.551045,0.26135,-1.467754 \backslash 0,0,0.458012$ $0.808245,0.040932 \backslash 0,0,0.666057,-0.853605,-2.0403 \backslash 0,0,-0.563758,-1.470$ $239,0.242878|C, 0,-1.966595,2.219387,-0.161168| C, 0,-2.367637,0.953751,-$ $0.595075 \backslash \mathrm{C}, 0,-3.619649,0.362264,-0.240258 \backslash \mathrm{C}, 0,-4.454386,1.078329,0.606$ $624 \mathrm{IH}, 0,-5.434563,0.668544,0.87057 \backslash \mathrm{C}, 0,-4.063849,2.329673,1.164711 \backslash \mathrm{C}, 0$ $,-2.795234,2.909012,0.796549 \mathrm{lC}, 0,-2.392808,4.118203,1.440626 \mathrm{lH}, 0,-1.41$ $4068,4.547023,1.207398 \mid \mathrm{C}, 0,-3.212086,4.73988,2.372301 \backslash \mathrm{H}, 0,-2.875571,5$. $660504,2.861508 \mathrm{IC}, 0,-4.478752,4.187974,2.703907 \backslash \mathrm{H}, 0,-5.118478,4.691594$ ,3.43676\C, $0,-4.891325,3.004307,2.112244 \backslash \mathrm{H}, 0,-5.855196,2.554807,2.3766$ $76|C, 0,-0.685059,2.798111,-0.655946| C, 0,0.504796,2.078775,-0.498161 \backslash C$, $0,1.791099,2.636597,-0.776692 \backslash \mathrm{C}, 0,1.843095,3.91822,-1.30974 \backslash \mathrm{H}, 0,2.8165$ $5,4.376143,-1.514886 \mid C, 0,0.661673,4.654206,-1.612211 \backslash \mathrm{C}, 0,-0.626515,4.0$ $89695,-1.293012 \mathrm{lC}, 0,-1.794995,4.830205,-1.647377 \backslash \mathrm{H}, 0,-2.780375,4.39960$ $8,-1.447143 \backslash \mathrm{C}, 0,-1.697187,6.072721,-2.256252 \backslash \mathrm{H}, 0,-2.608391,6.618021,-2$ $.52571 \backslash \mathrm{C}, 0,-0.426272,6.641251,-2.539926 \backslash \mathrm{H}, 0,-0.36306,7.625598,-3.01650$ 9IC, $0,0.727976,5.940406,-2.228256 \backslash \mathrm{H}, 0,1.714512,6.359079,-2.457858 \mathrm{IC}, 0$, $3.774629,-3.544839,-0.175494 \backslash \mathrm{C}, 0,3.543991,-4.376084,0.937725 \mathrm{lH}, 0,3.972$ $912,-5.382239,0.972327 \backslash \mathrm{C}, 0,2.764338,-3.912799,1.999535 \mathrm{H}, 0,2.578037,-4$ $.563024,2.859295 \backslash \mathrm{C}, 0,2.189021,-2.622127,1.94255 \backslash \mathrm{C}, 0,2.448818,-1.791997$ $, 0.832529 \backslash \mathrm{H}, 0,2.075962,-0.765306,0.810381 \backslash \mathrm{C}, 0,3.237292,-2.252002,-0.22$ $5055 \backslash \mathrm{H}, 0,3.435134,-1.595316,-1.073167 \backslash \mathrm{C}, 0,1.342938,-2.121864,3.048961 \backslash$ C, $0,1.766338,-2.359538,4.463359 \mathrm{H}, 0,1.387299,-1.576383,5.138423 \backslash \mathrm{H}, 0,2$. $864694,-2.404847,4.517205 \backslash \mathrm{H}, 0,1.371841,-3.332646,4.812067 \backslash \mathrm{N}, 0,0.271707$ $,-1.444086,2.715279 \mathrm{IH}, 0,0.006541,-1.396369,1.655398 \mathrm{IC}, 0,-0.671365,-0.7$ $75518,3.552856 \mathrm{IC}, 0,-1.129046,0.476902,3.097622 \backslash \mathrm{H}, 0,-0.742249,0.883048$, $2.158379 \backslash \mathrm{C}, 0,-2.068784,1.182139,3.857765 \backslash \mathrm{H}, 0,-2.412352,2.162061,3.5144$ 23IC $, 0,-2.576064,0.630707,5.045249 \backslash \mathrm{C}, 0,-2.141461,-0.633731,5.472693 \mathrm{lH}$, $0,-2.553448,-1.079194,6.384108 \mathrm{CC}, 0,-1.187899,-1.34541,4.731563 \mathrm{H}, 0,-0$. $883178,-2.345591,5.04757 \backslash \mathrm{C}, 0,3.046763,1.888075,-0.442535 \backslash \mathrm{C}, 0,3.865024$, $1.353119,-1.47258 \mid C, 0,3.447075,1.772284,0.918477 \backslash C, 0,5.089809,0.752124$ -1.124309IC $0,4.679289,1.161028,1.213088 \mid C, 0,5.524859,0.659222,0.2080$ $34 \mathrm{IH}, 0,5.722084,0.344792,-1.921663 \backslash \mathrm{H}, 0,5.004499,1.082852,2.256577 \backslash \mathrm{C}, 0$, $-4.029437,-0.960231,-0.815649 \backslash \mathrm{C}, 0,-4.067244,-2.124247,0.002419 \backslash \mathrm{C}, 0,-4$ $406805,-1.040691,-2.182637 \backslash C, 0,-4.533571,-3.32825,-0.55517 \backslash C, 0,-4.8565$ $7,-2.272928,-2.694249 \mathrm{IC}, 0,-4.937825,-3.426424,-1.898744 \mathrm{IH}, 0,-4.570117$, $-4.22262,0.076787 \mathrm{H}, 0,-5.15266,-2.33716,-3.748656 \mathrm{H}, 0,-3.320785,1.1816$ $86,5.629046 \backslash \mathrm{C}, 0,4.636258,-4.059352,-1.302137 \backslash \mathrm{C}, 0,-4.34272,0.169678,-3$. $115538 \mathrm{H}, 0,-3.978661,1.030525,-2.529803 \backslash \mathrm{C}, 0,-3.605204,-2.101368,1.4613$ $18 \mathrm{IC}, 0,-5.427713,-4.748307,-2.483495 \mathrm{H}, 0,-5.696597,-4.554802,-3.539081$ IC, $0,-4.800309,-2.014758,2.43373 \mathrm{H}, 0,-4.446337,-1.966934,3.479992 \mathrm{H}, 0$, $-5.41696,-1.119916,2.242859 \mathrm{H}, 0,-5.452832,-2.902286,2.333402 \mathrm{lC}, 0,-6.69$ $3209,-5.258394,-1.764501 \mathrm{H}, 0,-6.484358,-5.490468,-0.703796 \backslash \mathrm{H}, 0,-7.5004$ $49,-4.504749,-1.794498 \backslash \mathrm{H}, 0,-7.067377,-6.182083,-2.243037 \backslash \mathrm{C}, 0,-4.314575$ $,-5.816423,-2.472255 \backslash \mathrm{H}, 0,-4.662024,-6.747121,-2.957474 \backslash \mathrm{H}, 0,-3.417027,-$ $5.461918,-3.009657 \backslash \mathrm{H}, 0,-4.013919,-6.066976,-1.438188 \backslash \mathrm{C}, 0,-3.336388,-0$ $059596,-4.262368 \mathrm{H}, 0,-3.658332,-0.885585,-4.923282 \mathrm{H}, 0,-3.246275,0.851$ $478,-4.882494 \mathrm{IH}, 0,-2.338371,-0.302791,-3.860187 \backslash \mathrm{C}, 0,-5.738134,0.549178$ $,-3.651827 \backslash \mathrm{H}, 0,-5.677331,1.458872,-4.277145 \backslash \mathrm{H}, 0,-6.167421,-0.258114,-4$ $.273331 \mathrm{lH}, 0,-6.440988,0.750558,-2.823262 \mathrm{lC}, 0,6.869034,0.026349,0.56019$ $9 \mathrm{H}, 0,7.010784,0.153128,1.650111 \backslash \mathrm{C}, 0,3.462475,1.422722,-2.947511 \mathrm{VH}, 0,2$ $.425824,1.798468,-2.992756 \mathrm{IC}, 0,2.595833,2.367643,2.043315 \mathrm{|H}, 0,1.545475$ ,2.352766,1.707371\C,0,6.877447,-1.485808,0.258557\H,0,7.847019,-1.936 $183,0.540385 \mathrm{\backslash H}, 0,6.082776,-2.008217,0.817669 \backslash \mathrm{H}, 0,6.715685,-1.676417,-0$ $.816944 \backslash \mathrm{C}, 0,8.038129,0.738051,-0.150268 \mathrm{H}, 0,7.974831,0.615926,-1.24720$ $7 \mathrm{VH}, 0,8.042739,1.820383,0.071066 \mathrm{H}, 0,9.005913,0.31655,0.177771 \backslash \mathrm{C}, 0,2.6$ $57363,1.563723,3.35436 \mathrm{H}, 0,2.454138,0.493969,3.177542 \backslash \mathrm{H}, 0,3.64333,1.64$ $6002,3.846492 \backslash \mathrm{H}, 0,1.902554,1.941438,4.066781 \backslash \mathrm{C}, 0,2.978139,3.844424,2.2$ $83562 \mathrm{H}, 0,2.871043,4.438222,1.358832 \mathrm{H}, 0,2.329559,4.295375,3.057258 \mathrm{H}$, $0,4.027213,3.92648,2.62287 \backslash \mathrm{C}, 0,4.357704,2.418269,-3.714343 \backslash \mathrm{H}, 0,4.31963$ $1,3.425057,-3.261469 \mathrm{H}, 0,5.412578,2.086145,-3.71274 \mathrm{|H}, 0,4.030812,2.504$ $244,-4.766908 \backslash \mathrm{C}, 0,3.465181,0.032983,-3.616393 \backslash \mathrm{H}, 0,3.172674,0.124098,-4$ $.678965 \mathrm{IH}, 0,4.464516,-0.437872,-3.586033 \mathrm{H}, 0,2.7378,-0.62952,-3.119488$ IC $, 0,-2.712603,-3.309066,1.809444 \backslash \mathrm{H}, 0,-3.279507,-4.258013,1.794062 \backslash \mathrm{H}, 0$ $,-1.870146,-3.385582,1.103297 \backslash \mathrm{H}, 0,-2.300514,-3.191687,2.82754 \backslash \mathrm{H}, 0,-2.9$ $89799,-1.195334,1.591762 \mathrm{~F}, 0,5.861316,-4.454544,-0.856814 \mathrm{IF}, 0,4.083811$ $,-5.143494,-1.907801 \mathrm{IF}, 0,4.847391,-3.131227,-2.267282 \mathrm{IV}$ Version=ES64L-G 09RevD.01/State=1-AlHF=-3513.6171451 IRMSD=2.061e-09lDipole=0.3160675, $0.3493171,6.3937597 \backslash$ Quadrupole $=-12.9074242,0.2214301,12.6859941,-4.978$ 4625,5.5676663,-23.7363564|PG=C01 [X(C65H69F3N1O4P1)]॥@

\section{TRIP/2E Switching TS}

\section{Confo}

1\1|GINC-WORKER0ISPIRTPSSTPSSIdef2SVPIC65H69F3N1O4P1\JHIOEI29-Apr-2019 
IO\\p tpsstpss/def2svp int=ultrafine empiricaldispersion=gd3 scrf=(sm $\mathrm{d}$, solvent $=\mathrm{ch} 2 \mathrm{cl} 2$, read,externaliteration, dovacuum) \titlell0,1 $1 \mathrm{P}, 0,-0.1$ $26727,0.039424,-0.427595 \backslash 0,0,1.122372,0.953558,0.22631310,0,-1.277575$, $1.259786,-0.602067 \backslash 0,0,-0.523336,-0.940285,0.656727 \backslash 0,0,0.159356,-0.47$ $6635,-1.819212 \backslash \mathrm{C}, 0,0.564954,3.27472,-0.00941 \backslash \mathrm{C}, 0,1.425549,2.203494,-0$. $269942 \backslash \mathrm{C}, 0,2.672104,2.352344,-0.955731 \backslash \mathrm{C}, 0,3.012038,3.615648,-1.42058$ $\mathrm{H}, 0,3.969574,3.76342,-1.93125 \backslash \mathrm{C}, 0,2.128303,4.725661,-1.285174 \backslash \mathrm{C}, 0,0.87$ $6387,4.55797,-0.587492 \mathrm{C}, 0,-0.017267,5.669988,-0.526413 \mathrm{H}, 0,-0.986816$, $5.547464,-0.03477 \backslash \mathrm{C}, 0,0.320205,6.891616,-1.090391 \backslash \mathrm{H}, 0,-0.386196,7.7274$ $71,-1.03726 \backslash \mathrm{C}, 0,1.569928,7.065418,-1.743638 \mathrm{H}, 0,1.827534,8.036832,-2.1$ $79786 \backslash \mathrm{C}, 0,2.451519,6.000296,-1.841048 \backslash \mathrm{H}, 0,3.409915,6.115752,-2.360217 \backslash$ C, $0,-0.637072,3.054098,0.843971 \backslash \mathrm{C}, 0,-1.538597,2.03931,0.509706 \backslash \mathrm{C}, 0,-2$. $754296,1.804641,1.227332 \backslash \mathrm{C}, 0,-3.031488,2.621828,2.314477 \backslash \mathrm{H}, 0,-3.973926$ ,2.497224,2.857334\C, $0,-2.113133,3.616156,2.7608 \backslash \mathrm{C}, 0,-0.884253,3.82769$ $3,2.035475 \backslash \mathrm{C}, 0,0.050818,4.774349,2.554 \backslash \mathrm{H}, 0,1.007519,4.913955,2.042514 \backslash$ $\mathrm{C}, 0,-0.229717,5.50355,3.700339 \backslash \mathrm{H}, 0,0.506933,6.218974,4.082351 \backslash \mathrm{C}, 0,-1.4$ $60052,5.325011,4.387956 \mathrm{IH}, 0,-1.672511,5.911663,5.288449 \mathrm{IC}, 0,-2.37836,4$ $.3946,3.92766 \mathrm{WH}, 0,-3.321176,4.229625,4.461788 \mathrm{IC}, 0,-3.707911,0.742521,0$ $.76457 \backslash \mathrm{C}, 0,-3.810459,-0.496282,1.457817 \backslash \mathrm{C}, 0,-4.513469,0.9871,-0.378677$ $\mid \mathrm{C}, 0,-4.717653,-1.461167,0.983817 \backslash \mathrm{C}, 0,-5.409687,-0.010176,-0.808611 \backslash \mathrm{C}$, $0,-5.523754,-1.243845,-0.148223 \backslash \mathrm{H}, 0,-4.79679,-2.415575,1.51574 \backslash \mathrm{H}, 0,-6$. $032754,0.176304,-1.691891 \backslash \mathrm{C}, 0,3.561415,1.160903,-1.139782 \backslash \mathrm{C}, 0,3.756959$ $, 0.607056,-2.433399 \backslash \mathrm{C}, 0,4.199502,0.573675,-0.011185 \backslash \mathrm{C}, 0,4.60972,-0.505$ $623,-2.571978 \mathrm{IC}, 0,5.028239,-0.546659,-0.200941 \backslash \mathrm{C}, 0,5.25487,-1.099848,-$ $1.475596 \mathrm{H}, 0,4.772085,-0.930369,-3.570268 \mathrm{H}, 0,5.51937,-0.993052,0.6715$ $53 \mathrm{IC}, 0,4.055848,1.164869,1.393251 \backslash \mathrm{H}, 0,3.328292,1.991669,1.340114 \backslash \mathrm{C}, 0,3$ $.065719,1.171691,-3.677018 \mathrm{IC}, 0,6.161231,-2.312799,-1.673934 \backslash \mathrm{H}, 0,6.2690$ $61,-2.455423,-2.765843 \mid \mathrm{C}, 0,4.07867,1.850014,-4.6221 \mathrm{H}, 0,4.640077,2.648$ $903,-4.105173 \mathrm{lH}, 0,4.811855,1.119946,-5.012767 \backslash \mathrm{H}, 0,3.558926,2.302292,-5$ $.486941 \backslash \mathrm{C}, 0,5.525942,-3.596285,-1.098558 \mathrm{WH}, 0,4.523369,-3.772963,-1.526$ $82 \mathrm{H}, 0,6.155679,-4.476369,-1.32565 \mathrm{|H}, 0,5.421593,-3.531382,-0.001242 \backslash \mathrm{C}$, $0,7.570774,-2.086741,-1.09199 \mathrm{H}, 0,7.539758,-1.975452,0.00745 \mathrm{H}, 0,8.227$ $363,-2.94598,-1.321433 \mathrm{H}, 0,8.036886,-1.177579,-1.512361 \backslash \mathrm{C}, 0,3.51342,0$. $142287,2.409768 \mathrm{lH}, 0,2.549914,-0.27478,2.073623 \mathrm{lH}, 0,4.223696,-0.687707$, $2.563431 \mathrm{WH}, 0,3.353245,0.625467,3.391174 \mid \mathrm{C}, 0,5.393858,1.770977,1.86831 \backslash$ $\mathrm{H}, 0,5.272652,2.254528,2.855302 \backslash \mathrm{H}, 0,6.173441,0.992804,1.965637 \backslash \mathrm{H}, 0,5.76$ $2029,2.532045,1.156874 \backslash \mathrm{C}, 0,-6.50665,-2.305078,-0.636279 \backslash \mathrm{H}, 0,-6.9035,-1$ $.954115,-1.60761 \backslash \mathrm{C}, 0,-2.984238,-0.785342,2.712935 \backslash \mathrm{H}, 0,-2.107918,-0.116$ $009,2.682495 \backslash \mathrm{C}, 0,-4.436411,2.308541,-1.145392 \mathrm{H}, 0,-3.695381,2.948175,-$ $0.636755 \backslash \mathrm{C}, 0,-5.828334,-3.669326,-0.87526 \mathrm{IH}, 0,-5.434519,-4.094306,0.06$ $6066 \backslash \mathrm{H}, 0,-6.553185,-4.393641,-1.289821 \backslash \mathrm{H}, 0,-4.990138,-3.578151,-1.5872$ $87 \backslash \mathrm{C}, 0,-7.697827,-2.44047,0.335877 \backslash \mathrm{H}, 0,-8.212391,-1.472595,0.472921 \backslash \mathrm{H}$, $0,-8.435106,-3.17108,-0.045115 \backslash \mathrm{H}, 0,-7.358514,-2.787602,1.32948 \backslash \mathrm{C}, 0,-3$. $94362,2.1037,-2.591849 \mathrm{H}, 0,-3.803796,3.079395,-3.092643 \mathrm{H}, 0,-2.979041$, $1.568869,-2.604373 \mathrm{IH}, 0,-4.674644,1.526305,-3.1872 \mathrm{IC}, 0,-5.782029,3.0614$ $54,-1.110249 \mathrm{H}, 0,-6.110339,3.242233,-0.070849 \mathrm{H}, 0,-5.690491,4.040447,-$ $1.615638 \backslash \mathrm{H}, 0,-6.578002,2.491145,-1.623836 \backslash \mathrm{C}, 0,-3.7976,-0.470912,3.9874$ $41 \backslash \mathrm{H}, 0,-4.691095,-1.120193,4.050463 \backslash \mathrm{H}, 0,-3.185461,-0.644375,4.891849 \mathrm{H}$ $, 0,-4.142518,0.57687,4.005848 \mathrm{IC}, 0,-2.445339,-2.227208,2.763529 \mathrm{H}, 0,-1$. $746052,-2.337831,3.612527 \mathrm{H}, 0,-3.251979,-2.970118,2.905597 \mathrm{H}, 0,-1.8934$ $44,-2.460962,1.841216 \backslash \mathrm{C}, 0,2.238764,0.098588,-4.414052 \mathrm{H}, 0,1.711329,0.5$ $52193,-5.273914 \mathrm{IH}, 0,2.877604,-0.713339,-4.808015 \mathrm{H}, 0,1.486946,-0.33466$ $,-3.732971 \backslash \mathrm{H}, 0,2.35579,1.945617,-3.339931 \backslash \mathrm{C}, 0,2.470902,-3.847655,2.227$ 989IC, $0,1.403329,-4.677978,2.613104 \mathrm{IH}, 0,1.445933,-5.227791,3.557514 \mathrm{IC}$, $0,0.28701,-4.800375,1.784289 \mathrm{H}, 0,-0.541542,-5.437941,2.102578 \backslash \mathrm{C}, 0,0.21$ $5969,-4.080365,0.568442 \backslash \mathrm{C}, 0,1.303201,-3.253965,0.193346 \mathrm{H}, 0,1.29202,-2$ $.710877,-0.756492 \mathrm{lC}, 0,2.423249,-3.139493,1.017105 \mathrm{VH}, 0,3.254463,-2.4997$ $16,0.71359 \backslash \mathrm{C}, 0,-0.960511,-4.186169,-0.310699 \backslash \mathrm{C}, 0,-1.822009,-5.404904,-$ $0.304773 \mathrm{H}, 0,-1.992835,-5.717057,0.738432 \backslash \mathrm{H}, 0,-1.306077,-6.241756,-0.8$ $09262 \backslash \mathrm{H}, 0,-2.791678,-5.226541,-0.790873 \mathrm{WN}, 0,-1.215073,-3.15617,-1.0925$ $93 \mathrm{lH}, 0,-0.715147,-2.269969,-0.85883 \backslash \mathrm{C}, 0,-2.132373,-3.042968,-2.175157 \backslash$ $\mathrm{C}, 0,-2.296664,-4.088646,-3.10463 \backslash \mathrm{H}, 0,-1.718634,-5.011408,-3.009131 \backslash \mathrm{C}, 0$ ,-3.178174,-3.916596,-4.178429)H,0,-3.305067,-4.726065,-4.904628\C,0,$3.875709,-2.708045,-4.335567 \backslash \mathrm{C}, 0,-3.687856,-1.665267,-3.414718 \mathrm{IH}, 0,-4$. $226671,-0.721642,-3.532226 \backslash \mathrm{C}, 0,-2.816667,-1.823016,-2.331426 \backslash \mathrm{H}, 0,-2.68$ $8982,-1.020842,-1.600182 \backslash \mathrm{H}, 0,-4.559968,-2.575996,-5.180118 \backslash \mathrm{C}, 0,3.65043$ $1,-3.691314,3.15736 \backslash F, 0,3.965689,-4.854248,3.779991 \backslash F, 0,4.76964,-3.267$ 415,2.51348|F,0,3.397941,-2.782498,4.137994IIVersion=ES64L-G09RevD.01। State=1-AlHF=-3513.6072361 IRMSD =4.694e-09IDipole $=-2.3406352,-4.8416816$ ,-0.8551587\Quadrupole=-15.7819396,34.2362665,-18.4543269,17.4995955,3 $.8230971,9.5584936 / P G=C 01[X(C 65 H 69 F 3 N 104 P 1)] 1 @$

\section{Conf1}

1\1|GINC-WORKER0ISPIRTPSSTPSSIdef2SVPIC65H69F3N1O4P1\JHIOEI03-May-2019 IOll\#p tpsstpss/def2svp int=ultrafine empiricaldispersion=gd3 scrf $=(\mathrm{sm}$ $\mathrm{d}$, solvent=ch2cl2, read,externaliteration, dovacuum) \titlell0,1 $\backslash \mathrm{P}, 0,-0.1$ $557,0.028222,-0.404327 \backslash 0,0,1.034305,1.017155,0.250479 \backslash 0,0,-1.355159,1$. 
$188475,-0.6433 \backslash 0,0,-0.530209,-0.936561,0.700594 \backslash 0,0,0.183359,-0.514408$ $,-1.774104 \backslash \mathrm{C}, 0,0.393253,3.299134,-0.110794 \backslash \mathrm{C}, 0,1.30526,2.254665,-0.292$ $474 \backslash \mathrm{C}, 0,2.568805,2.423345,-0.942064 \mid \mathrm{C}, 0,2.862148,3.669558,-1.478649 \mathrm{H}$, $0,3.828386,3.833796,-1.967563|C, 0,1.92649,4.744088,-1.429899| C, 0,0.667$ $692,4.562914,-0.747408 \backslash \mathrm{C}, 0,-0.267846,5.641506,-0.757497 \backslash \mathrm{H}, 0,-1.240211$, $5.50697,-0.274551 \backslash \mathrm{C}, 0,0.031208,6.843353,-1.382511 \backslash \mathrm{H}, 0,-0.707192,7.6528$ 43,-1.383637\C,0,1.282976,7.030157,-2.028179\H,0,1.508835,7.984999,-2. $515495 \backslash \mathrm{C}, 0,2.206662,5.996993,-2.054238 \mathrm{H}, 0,3.168592,6.122317,-2.564482$ IC, $0,-0.816325,3.069646,0.730805 \backslash C, 0,-1.667015,2.001501,0.430859 \backslash C, 0,-$ $2.878553,1.738948,1.145862 \backslash \mathrm{C}, 0,-3.205035,2.58562,2.195993 \backslash \mathrm{H}, 0,-4.14491$ $4,2.437037,2.737278|C, 0,-2.341262,3.642386,2.606023| C, 0,-1.116176,3.88$ $3703,1.883101 \backslash \mathrm{C}, 0,-0.236643,4.89901,2.368046 \mathrm{H}, 0,0.716745,5.067136,1.8$ $5933 \backslash \mathrm{C}, 0,-0.566645,5.662152,3.478582 \backslash \mathrm{H}, 0,0.128186,6.430922,3.834421 \backslash \mathrm{C}$ $0,-1.793328,5.450725,4.163149 \backslash \mathrm{H}, 0,-2.044876,6.064018,5.035362 \backslash \mathrm{C}, 0,-2.6$ $58104,4.455761,3.735689 \mathrm{H}, 0,-3.597431,4.266446,4.267956 \mathrm{IC}, 0,-3.77964,0$ $.618116,0.716539|\mathrm{C}, 0,-3.829991,-0.600796,1.44969| \mathrm{C}, 0,-4.590889,0.78875$ $3,-0.436324 \backslash C, 0,-4.691504,-1.620095,1.00492 \backslash C, 0,-5.439879,-0.261133,-0$ $.835948 \mathrm{IC}, 0,-5.502026,-1.476216,-0.135516 \mathrm{IH}, 0,-4.73146,-2.558536,1.568$ $422 \backslash \mathrm{H}, 0,-6.067118,-0.132375,-1.726602 \backslash \mathrm{C}, 0,3.530456,1.275315,-0.986271 \backslash$ C, $0,3.82094,0.619374,-2.214565 \backslash C, 0,4.137424,0.825152,0.218055 \backslash C, 0,4.71$ $9686,-0.463795,-2.211742 \backslash \mathrm{C}, 0,5.030773,-0.260542,0.166161 \backslash \mathrm{C}, 0,5.332317$, $-0.925954,-1.03385 \backslash \mathrm{H}, 0,4.938829,-0.966815,-3.160942 \backslash \mathrm{H}, 0,5.5029,-0.6064$ $41,1.092955 \backslash \mathrm{C}, 0,3.872417,1.496681,1.567231 \backslash \mathrm{H}, 0,3.122172,2.289868,1.412$ 292\C,0,3.181291,1.045914,-3.538316।C,0,6.298984,-2.107645,-1.0475\H,0 $, 6.571764,-2.312424,0.004658 \mathrm{IC}, 0,4.225817,1.683377,-4.477867 \backslash \mathrm{H}, 0,4.721$ $461,2.548645,-4.002138 \mathrm{H}, 0,5.010918,0.954727,-4.75356 \mathrm{H}, 0,3.747185,2.0$ $33534,-5.411077 \backslash \mathrm{C}, 0,7.594003,-1.764509,-1.812886 \mathrm{IH}, 0,8.086158,-0.87211$ $5,-1.386459 \mathrm{H}, 0,8.310429,-2.605449,-1.767966 \backslash \mathrm{H}, 0,7.382266,-1.557994,-2$ $.878364 \mathrm{IC}, 0,5.642027,-3.383376,-1.613068 \mathrm{IH}, 0,5.355925,-3.252793,-2.672$ $831 \mathrm{H}, 0,6.342203,-4.23703,-1.557532 \mathrm{H}, 0,4.732707,-3.649656,-1.046039 \mathrm{C}$ $, 0,3.288852,0.507069,2.59567 \backslash \mathrm{H}, 0,2.377607,0.025965,2.203474 \mathrm{IH}, 0,4.0172$ $31,-0.279703,2.857517 \backslash \mathrm{H}, 0,3.022097,1.03747,3.528488 \mathrm{IC}, 0,5.147404,2.179$ $866,2.105152 \mathrm{H}, 0,4.934165,2.711636,3.050783 \mathrm{H}, 0,5.944468,1.440346,2.30$ $6374 \backslash \mathrm{H}, 0,5.541779,2.915111,1.380675 \backslash \mathrm{C}, 0,-6.436724,-2.594424,-0.589682 \backslash$ $\mathrm{H}, 0,-6.841123,-2.296143,-1.575466 \mathrm{IC}, 0,-2.998057,-0.813155,2.71614 \mathrm{IH}, 0$, $-2.146913,-0.113425,2.663951 \backslash \mathrm{C}, 0,-4.56978,2.086302,-1.246454 \mathrm{H}, 0,-3.86$ $8821,2.779161,-0.750966 \backslash \mathrm{C}, 0,-5.702783,-3.938072,-0.773872 \mathrm{WH}, 0,-5.29978$ $9,-4.312974,0.184768 \mathrm{H}, 0,-6.395246,-4.705015,-1.166459 \mathrm{H}, 0,-4.863237,-$ $3.839689,-1.483405 \mathrm{IC}, 0,-7.62816,-2.742196,0.380478 \mathrm{H}, 0,-8.179365,-1.79$ $0317,0.482856 \mathrm{H}, 0,-8.335141,-3.512357,0.020536 \mathrm{H}, 0,-7.282093,-3.04317$, $1.386737 \backslash \mathrm{C}, 0,-4.046794,1.85775,-2.678853 \backslash \mathrm{H}, 0,-3.944664,2.821913,-3.210$ $151 \mathrm{lH}, 0,-3.058072,1.369159,-2.659884 \backslash \mathrm{H}, 0,-4.740689,1.227071,-3.264501 \backslash$ $\mathrm{C}, 0,-5.951515,2.771079,-1.251809 \mathrm{H}, 0,-6.299317,2.972195,-0.222491 \backslash \mathrm{H}, 0$, $-5.904079,3.734575,-1.791845 \mathrm{IH}, 0,-6.712577,2.143342,-1.750869 \mathrm{IC}, 0,-3.8$ $28041,-0.483544,3.976057 \backslash \mathrm{H}, 0,-4.698113,-1.161798,4.058287 \backslash \mathrm{H}, 0,-3.21500$ $3,-0.603915,4.88848 \mathrm{H}, 0,-4.209753,0.551407,3.956255 \backslash \mathrm{C}, 0,-2.407022,-2.2$ $32036,2.820345 \backslash \mathrm{H}, 0,-1.695765,-2.281563,3.665131 \backslash \mathrm{H}, 0,-3.185242,-2.99627$ $3,3.002584 \backslash \mathrm{H}, 0,-1.856094,-2.485125,1.90232 \backslash \mathrm{C}, 0,2.442816,-0.120577,-4.2$ $26076 \backslash \mathrm{H}, 0,1.961765,0.232425,-5.157341 \backslash \mathrm{H}, 0,3.134351,-0.938971,-4.499565$ $\mathrm{IH}, 0,1.659541,-0.519405,-3.559581 \mathrm{|H}, 0,2.423944,1.815169,-3.311775 \backslash \mathrm{C}, 0$, $2.753475,-3.666009,2.220085 \backslash \mathrm{C}, 0,1.744699,-4.539596,2.664009 \backslash \mathrm{H}, 0,1.8582$ $41,-5.078951,3.608723 \backslash \mathrm{C}, 0,0.597825,-4.723986,1.889795 \backslash \mathrm{H}, 0,-0.182497,-5$ $.398351,2.251006 \mathrm{IC}, 0,0.438043,-4.027124,0.668923 \backslash \mathrm{C}, 0,1.46248,-3.14812$, $0.240117 \backslash \mathrm{H}, 0,1.383166,-2.617009,-0.713381 \backslash \mathrm{C}, 0,2.611394,-2.968032,1.010$ $755 \backslash \mathrm{H}, 0,3.39295,-2.287016,0.666075 \backslash \mathrm{C}, 0,-0.754834,-4.224603,-0.171695 \backslash \mathrm{C}$ $, 0,-1.542126,-5.491141,-0.109934 \backslash \mathrm{H}, 0,-1.666873,-5.784595,0.945374 \backslash \mathrm{H}, 0$, $-0.99132,-6.309932,-0.607363 \mathrm{H}, 0,-2.533085,-5.382143,-0.572986 \mathrm{WN}, 0,-1$. $085749,-3.235572,-0.977848 \mathrm{H}, 0,-0.633327,-2.31637,-0.779619 \mathrm{lC}, 0,-2.017$ $458,-3.206336,-2.052753 \backslash \mathrm{C}, 0,-2.147414,-4.295525,-2.936959 \backslash \mathrm{H}, 0,-1.53488$ $8,-5.191181,-2.807001 \backslash \mathrm{C}, 0,-3.037157,-4.20052,-4.013379 \mathrm{WH}, 0,-3.136433,-$ $5.043912,-4.704422 \backslash \mathrm{C}, 0,-3.77718,-3.024977,-4.219234 \backslash \mathrm{C}, 0,-3.624733,-1.9$ $38685,-3.343391 \mathrm{H}, 0,-4.196524,-1.020355,-3.498816 \mathrm{IC}, 0,-2.746607,-2.019$ $609,-2.25726 \mathrm{H}, 0,-2.646388,-1.182374,-1.5617 \mathrm{H}, 0,-4.467136,-2.952931,-$ $5.066411 \mathrm{IC}, 0,3.98436,-3.469324,3.072543 \mathrm{IF}, 0,4.412351,-4.634661,3.62075$ 4IF,0,5.024639,-2.952268,2.371115\F,0,3.749097,-2.619159,4.109412IIVer sion=ES64L-G09RevD.01।State=1-AlHF=-3513.6070822/RMSD=4.627e-09|Dipole $=-2.0930171,-4.9468465,-0.726474 \mid$ Quadrupole $=-17.7116987,36.4686815,-18$ .7569828,15.2310487,3.3327362,7.6537515IPG=C01 [X(C65H69F3N1O4P1)]॥@

\section{Conf2}

1\1|GINC-WORKER1ISPIRTPSSTPSSIdef2SVPIC65H69F3N1O4P1।JHIOEI29-Apr-2019

IO\॥p tpsstpss/def2svp int=ultrafine empiricaldispersion=gd3 scrf=(sm $\mathrm{d}$,solvent=ch2cl2,read,externaliteration, dovacuum) \ltitlell0,1\P,0,-0.2 $36562,-0.045898,-0.397723 \backslash 0,0,0.904678,1.033761,0.208701 \backslash 0,0,-1.51622$, $1.028515,-0.607933 \backslash 0,0,-0.512511,-1.037434,0.712839 \backslash 0,0,0.113418,-0.56$ $7018,-1.772489|C, 0,0.136418,3.254735,-0.270969| C, 0,1.091063,2.244199,-$ 
$0.429053 \backslash \mathrm{C}, 0,2.308262,2.431029,-1.156682 \backslash \mathrm{C}, 0,2.523652,3.661757,-1.7614$ $64 \mathrm{IH}, 0,3.461859,3.842531,-2.296178 \mid \mathrm{C}, 0,1.547311,4.698722,-1.720239 \backslash \mathrm{C}, 0$ $, 0.322501,4.492116,-0.986711 \backslash \mathrm{C}, 0,-0.668644,5.519444,-1.025277 \backslash \mathrm{H}, 0,-1.6$ $20374,5.361064,-0.509633 \backslash \mathrm{C}, 0,-0.44667,6.702754,-1.714347 \backslash \mathrm{H}, 0,-1.225241$ ,7.473417,-1.73428\C,0,0.778094,6.921123,-2.400713।H,0,0.943776,7.8627 $81,-2.93553 \backslash \mathrm{C}, 0,1.750601,5.933654,-2.407053 \mathrm{IH}, 0,2.690793,6.080322,-2.9$ $51079 \mid \mathrm{C}, 0,-1.028296,3.017959,0.627958 \backslash \mathrm{C}, 0,-1.826385,1.887144,0.428713$ C, $0,-2.990881,1.604768,1.210678 \backslash \mathrm{C}, 0,-3.332338,2.504537,2.211264 \backslash \mathrm{H}, 0,-4$ $.238403,2.335486,2.801935 \backslash \mathrm{C}, 0,-2.521347,3.636231,2.514032 \backslash \mathrm{C}, 0,-1.33467$ ,3.891031,1.734328\C, $0,-0.497637,4.982444,2.119314 \backslash \mathrm{H}, 0,0.43003,5.16458$ $8,1.569596 \backslash \mathrm{C}, 0,-0.83612,5.803813,3.18485 \backslash \mathrm{H}, 0,-0.17429,6.631298,3.46354$ $7 \backslash \mathrm{C}, 0,-2.028946,5.577497,3.92252 \mathrm{H}, 0,-2.288806,6.237367,4.757489 \backslash \mathrm{C}, 0,-$ $2.849202,4.509766,3.594575 \mathrm{H}, 0,-3.760861,4.309254,4.169092 \mathrm{lC}, 0,-3.8176$ $73,0.385815,0.928676 \backslash \mathrm{C}, 0,-3.787291,-0.724325,1.815908 \backslash \mathrm{C}, 0,-4.646409,0$. $352425,-0.225963 \mid C, 0,-4.610031,-1.832621,1.536261 \backslash C, 0,-5.443093,-0.782$ $296,-0.464568 \mathrm{IC}, 0,-5.451046,-1.882604,0.412208 \mathrm{IH}, 0,-4.604621,-2.687472$ ,2.223077\} \backslash \mathrm { H } , 0 , - 6 . 0 8 6 7 7 2 , - 0 . 7 9 9 1 3 8 , - 1 . 3 5 2 0 6 3 \backslash \mathrm { C } , 0 , 3 . 3 2 9 0 7 9 , 1 . 3 3 4 8 3 5 , - 1 . 2 08798IC,0,3.541032,0.603182,-2.407464IC,0,4.100043,1.043532,-0.050114 C, $0,4.546428,-0.38295,-2.428083|C, 0,5.080818,0.037022,-0.117228| C, 0,5$. $329799,-0.682172,-1.301014 \mathrm{H}, 0,4.726485,-0.941431,-3.354682 \backslash \mathrm{H}, 0,5.6732$ $51,-0.182341,0.778561 \backslash \mathrm{C}, 0,3.923831,1.833099,1.249098 \backslash \mathrm{H}, 0,3.09951,2.550$ $103,1.098758 \backslash \mathrm{C}, 0,2.722742,0.871217,-3.672823 \backslash \mathrm{C}, 0,6.40206,-1.767185,-1$. $370679 \backslash \mathrm{H}, 0,6.516078,-2.03477,-2.438142 \backslash \mathrm{C}, 0,3.541416,1.685531,-4.696952$ $\mathrm{IH}, 0,3.888569,2.642932,-4.270858 \mathrm{H}, 0,4.433052,1.11978,-5.0263 \mathrm{H}, 0,2.93$ $2058,1.910679,-5.591685 \backslash \mathrm{C}, 0,5.962923,-3.041035,-0.617914 \mathrm{IH}, 0,5.000351$, $-3.421018,-1.003573 \backslash \mathrm{H}, 0,6.718457,-3.840324,-0.732414 \backslash \mathrm{H}, 0,5.841527,-2.8$ $42362,0.461284 \backslash \mathrm{C}, 0,7.770775,-1.271831,-0.863884 \backslash \mathrm{H}, 0,7.739579,-1.03336$, $0.215032 \mathrm{H}, 0,8.541954,-2.050161,-1.009857 \backslash \mathrm{H}, 0,8.093612,-0.363328,-1.40$ $3232 \mathrm{IC}, 0,3.531986,0.930157,2.434056 \mathrm{H}, 0,2.612557,0.364304,2.209968 \mathrm{H}, 0$ ,4.335683,0.214597,2.678504\H,0,3.346232,1.541903,3.336083।C,0,5.18821 $9,2.659229,1.565898 \mathrm{H}, 0,5.033078,3.272306,2.472691 \mathrm{H}, 0,6.06062,2.00409$ $7,1.745816 \mathrm{IH}, 0,5.439804,3.338303,0.731315 \mathrm{IC}, 0,-6.356397,-3.088746,0.17$ $3003 \mathrm{IH}, 0,-6.190716,-3.786456,1.015538 \backslash \mathrm{C}, 0,-2.915359,-0.732109,3.073815$ $\mathrm{IH}, 0,-2.159449,0.062063,2.951015 \backslash \mathrm{C}, 0,-4.725978,1.542102,-1.184907 \backslash \mathrm{H}, 0$, $-4.017229,2.308352,-0.828277 \backslash \mathrm{C}, 0,-7.844955,-2.683964,0.187495 \backslash \mathrm{H}, 0,-8.0$ $78355,-1.993741,-0.644108 \mathrm{H}, 0,-8.492834,-3.572965,0.078081 \mathrm{H}, 0,-8.1119$ $97,-2.178329,1.132775 \backslash \mathrm{C}, 0,-5.99832,-3.836377,-1.1267 \backslash \mathrm{H}, 0,-4.941665,-4$. $156472,-1.12568 \backslash \mathrm{H}, 0,-6.630526,-4.735968,-1.243219 \backslash \mathrm{H}, 0,-6.157644,-3.195$ $992,-2.013131 \backslash \mathrm{C}, 0,-4.303455,1.166589,-2.618207 \backslash \mathrm{H}, 0,-4.296355,2.064227$, $-3.263617 \backslash \mathrm{H}, 0,-3.290104,0.73129,-2.62572 \backslash \mathrm{H}, 0,-5.003633,0.439409,-3.068$ $701 \backslash \mathrm{C}, 0,-6.130122,2.18092,-1.158562 \backslash \mathrm{H}, 0,-6.410424,2.48196,-0.133032 \mathrm{H}$, $0,-6.159773,3.081209,-1.799732 \mathrm{H}, 0,-6.899228,1.477674,-1.528137 \backslash \mathrm{C}, 0,-3$ $.759039,-0.404166,4.324756 \mathrm{H}, 0,-4.525478,-1.183423,4.495419 \backslash \mathrm{H}, 0,-3.118$ $647,-0.354096,5.224932 \backslash \mathrm{H}, 0,-4.280854,0.563038,4.223642 \backslash \mathrm{C}, 0,-2.150654$,$2.055985,3.267906 \backslash \mathrm{H}, 0,-1.462056,-1.969444,4.128811 \backslash \mathrm{H}, 0,-2.83244,-2.900$ $768,3.477715 \backslash \mathrm{H}, 0,-1.551697,-2.283068,2.37257 \backslash \mathrm{C}, 0,2.178236,-0.421069,-4$ $.311709 \backslash \mathrm{H}, 0,1.535029,-0.170271,-5.175581 \backslash \mathrm{H}, 0,2.990827,-1.071116,-4.685$ $221 \mathrm{H}, 0,1.56946,-0.979699,-3.582839 \mathrm{H}, 0,1.849256,1.476626,-3.375572 \mathrm{lC}$, $0,2.973319,-3.287961,2.437882 \backslash \mathrm{C}, 0,2.143052,-4.388319,2.709381 \backslash \mathrm{H}, 0,2.25$ $7674,-4.9484,3.641225 \backslash \mathrm{C}, 0,1.168968,-4.766813,1.782596 \mathrm{IH}, 0,0.519495,-5$ $615164,2.013483 \backslash \mathrm{C}, 0,0.998704,-4.033906,0.585349 \mid \mathrm{C}, 0,1.847161,-2.930653$ $, 0.324634 \mathrm{IH}, 0,1.76149,-2.369669,-0.611014 \backslash \mathrm{C}, 0,2.831161,-2.564854,1.242$ $324 \backslash \mathrm{H}, 0,3.485974,-1.719554,1.020515 \backslash \mathrm{C}, 0,-0.051156,-4.393746,-0.384685 \backslash$ $\mathrm{C}, 0,-0.489365,-5.809127,-0.563172 \backslash \mathrm{H}, 0,-0.510855,-6.319714,0.412136 \mathrm{H}, 0$ $, 0.24692,-6.348007,-1.187716 \mathrm{WH}, 0,-1.479398,-5.877378,-1.03752 \mathrm{WN}, 0,-0.5$ $72144,-3.410173,-1.087105 \backslash \mathrm{H}, 0,-0.332561,-2.4392,-0.788518 \backslash \mathrm{C}, 0,-1.48571$ $,-3.45918,-2.180579 \mid \mathrm{C}, 0,-1.320962,-4.37742,-3.23397 \mathrm{H}, 0,-0.482618,-5.0$ $78746,-3.231439 \mathrm{IC}, 0,-2.219383,-4.350686,-4.308275 \backslash \mathrm{H}, 0,-2.091262,-5.059$ $248,-5.133066 \backslash C, 0,-3.265199,-3.414662,-4.334149 \mid C, 0,-3.408247,-2.49220$ $8,-3.28491 \backslash \mathrm{H}, 0,-4.219143,-1.759295,-3.300753 \backslash \mathrm{C}, 0,-2.519127,-2.504153$,$2.205179 \backslash \mathrm{H}, 0,-2.632967,-1.797036,-1.379076 \backslash \mathrm{H}, 0,-3.964277,-3.397814,-5$ $17681 \backslash \mathrm{C}, 0,3.996076,-2.849463,3.458565 \mathrm{~F}, 0,4.386048,-3.863512,4.267653 \mathrm{I}$ $\mathrm{F}, 0,5.119646,-2.34756,2.876751 \mathrm{IF}, 0,3.511337,-1.86847,4.267122 \mathrm{IV}$ Version $=E S 64 \mathrm{~L}-\mathrm{G} 09 \mathrm{RevD} .01 \mathrm{IState}=1-\mathrm{AlHF}=-3513.6051754 \mathrm{IRMSD}=4.412 \mathrm{e}-09 \mathrm{IDipole}=-0$. $984357,-5.2731299,-1.10842551$ Quadrupole $=-20.6458054,40.6653368,-20.019$ $5315,5.6342927,1.3009998,11.1672736 \mathrm{PG}=\mathrm{C} 01$ [X(C65H69F3N1O4P1)]॥@

\section{Conf3}

1\1\GINC-WORKER0ISPIRTPSSTPSSIdef2SVPIC65H69F3N1O4P1\JHIOEI29-Apr-2019 10।॥p tpsstpss/def2svp int=ultrafine empiricaldispersion=gd3 scrf $=(\mathrm{sm}$ $\mathrm{d}$, solvent $=\mathrm{ch} 2 \mathrm{cl} 2$, read,externaliteration, dovacuum) \title $\| \mathbf{0}, 1 \backslash \mathrm{P}, 0,-0.2$ $15755,-0.106495,-0.26437 \backslash 0,0,0.805073,1.097644,0.31751910,0,-1.591082$, $0.823352,-0.555867 \backslash 0,0,-0.420926,-1.072536,0.880618 \backslash 0,0,0.223251,-0.62$ $9931,-1.617047 \backslash C, 0,-0.155101,3.208807,-0.278492 \backslash C, 0,0.897106,2.292495$, $-0.367354 \backslash \mathrm{C}, 0,2.114161,2.569746,-1.064406 \backslash \mathrm{C}, 0,2.218561,3.783186,-1.731$ $222 \mathrm{H}, 0,3.14933,4.034092,-2.250631 \backslash \mathrm{C}, 0,1.137372,4.710568,-1.775643 \backslash \mathrm{C}, 0$ $,-0.076124,4.422921,-1.050294 \backslash \mathrm{C}, 0,-1.160512,5.346333,-1.152941 \backslash \mathrm{H}, 0,-2$. 
$100214,5.124443,-0.638409 \backslash \mathrm{C}, 0,-1.042006,6.508075,-1.901674 \backslash \mathrm{H}, 0,-1.8897$ $88,7.198734,-1.970355 \backslash \mathrm{C}, 0,0.166643,6.806822,-2.586443 \mathrm{lH}, 0,0.249618,7.7$ $30859,-3.169051 \backslash \mathrm{C}, 0,1.231401,5.921663,-2.525412 \backslash \mathrm{H}, 0,2.162998,6.132361$ $-3.063047 \backslash \mathrm{C}, 0,-1.313749,2.897991,0.605278 \backslash \mathrm{C}, 0,-2.009285,1.697883,0.428$ $8 \mathrm{IC}, 0,-3.18342,1.362973,1.173497 \backslash \mathrm{C}, 0,-3.625507,2.267193,2.129945 \backslash \mathrm{H}, 0,-$ $4.544937,2.056552,2.685873 \backslash \mathrm{C}, 0,-2.908569,3.463228,2.422736 \backslash \mathrm{C}, 0,-1.7212$ $88,3.781887,1.668398 \backslash \mathrm{C}, 0,-0.982585,4.948473,2.033062 \backslash \mathrm{H}, 0,-0.05555,5.18$ $0156,1.500733 \backslash \mathrm{C}, 0,-1.414579,5.777551,3.057891 \backslash \mathrm{H}, 0,-0.826738,6.663297,3$ $.323141 \mathrm{IC}, 0,-2.608064,5.484422,3.770944 \mathrm{IH}, 0,-2.942199,6.151213,4.57340$ $3 \backslash \mathrm{C}, 0,-3.334666,4.345664,3.460803 \backslash \mathrm{H}, 0,-4.245525,4.096217,4.017112 \backslash \mathrm{C}, 0$, $-3.948558,0.107189,0.875488|C, 0,-3.868803,-1.014306,1.743739| C, 0,-4.79$ $4709,0.067099,-0.266001 \backslash \mathrm{C}, 0,-4.668601,-2.139908,1.465276 \backslash \mathrm{C}, 0,-5.566401$ $,-1.084703,-0.504806 \backslash \mathrm{C}, 0,-5.533473,-2.194154,0.359387 \backslash \mathrm{H}, 0,-4.62818,-3$. $003453,2.139665 \mathrm{IH}, 0,-6.226166,-1.106456,-1.380279 \mathrm{IC}, 0,3.255502,1.59861$ $7,-1.013509 \mid \mathrm{C}, 0,3.607774,0.833487,-2.159647 \backslash \mathrm{C}, 0,4.007545,1.471153,0.18$ 4839IC $0,4.723838,-0.020732,-2.088774 \backslash \mathrm{C}, 0,5.120859,0.609281,0.201149 \mathrm{IC}$ $, 0,5.500935,-0.142635,-0.92284 \backslash \mathrm{H}, 0,4.996932,-0.604748,-2.975048 \backslash \mathrm{H}, 0,5$. $707043,0.512397,1.122601 \backslash \mathrm{C}, 0,3.646134,2.239182,1.457609 \backslash \mathrm{H}, 0,2.77115,2$. $872635,1.234487 \backslash \mathrm{C}, 0,2.820646,0.934106,-3.468423 \backslash \mathrm{C}, 0,6.716136,-1.06535$, $-0.868853 \mathrm{|H}, 0,7.123067,-1.001906,0.15736 \backslash \mathrm{C}, 0,3.594299,1.773033,-4.5079$ $22 \mathrm{H}, 0,3.825462,2.782141,-4.123993 \mathrm{H}, 0,4.551255,1.285705,-4.772915 \mathrm{lH}, 0$ ,3.002681,1.886583,-5.435152\C,0,7.820077,-0.601155,-1.841175\H,0,8.11 $6362,0.443588,-1.638479 \backslash \mathrm{H}, 0,8.718673,-1.237821,-1.743757 \backslash \mathrm{H}, 0,7.47711$,$0.659217,-2.890763 \mathrm{IC}, 0,6.333497,-2.537114,-1.125652 \backslash \mathrm{H}, 0,5.924411,-2.67$ $3875,-2.143704 \mathrm{H}, 0,7.21987,-3.190703,-1.028809 \mathrm{H}, 0,5.573842,-2.88306,-$ $0.403866 \backslash \mathrm{C}, 0,3.239937,1.27877,2.594729 \backslash \mathrm{H}, 0,2.409898,0.625218,2.278455 \backslash$ $\mathrm{H}, 0,4.087441,0.643146,2.90619 \mathrm{H}, 0,2.907581,1.851799,3.480209 \mathrm{CC}, 0,4.784$ $869,3.181753,1.898248 \mathrm{H}, 0,4.478726,3.769538,2.783171 \mathrm{H}, 0,5.695492,2.61$ $6988,2.170242 \backslash \mathrm{H}, 0,5.051505,3.889908,1.093096 \mid \mathrm{C}, 0,-6.424048,-3.412612,0$ $.126616 \mathrm{lH}, 0,-6.22807,-4.117733,0.956333 \mathrm{IC}, 0,-2.967237,-1.009202,2.9799$ $05 \mathrm{H}, 0,-2.205772,-0.226729,2.820292 \mathrm{CC}, 0,-4.917064,1.263502,-1.2122 \backslash \mathrm{H}, 0$ $,-4.232211,2.049067,-0.850705 \backslash \mathrm{C}, 0,-7.917837,-3.030287,0.181328 \mathrm{lH}, 0,-8$. $182025,-2.337361,-0.638761 \backslash \mathrm{H}, 0,-8.554877,-3.928129,0.08087 \backslash \mathrm{H}, 0,-8.1688$ $52,-2.535825,1.136871 \backslash \mathrm{C}, 0,-6.086637,-4.139273,-1.190255 \backslash \mathrm{H}, 0,-5.028467$, $-4.452418,-1.213875 \backslash \mathrm{H}, 0,-6.715105,-5.041444,-1.306981 \backslash \mathrm{H}, 0,-6.267902,-3$ $.48772,-2.064439 \mathrm{IC}, 0,-4.484565,0.913985,-2.649278 \mathrm{IH}, 0,-4.51814,1.81428$ 2,-3.290017\H,0,-3.453276,0.522432,-2.663178\H,0,-5.152892,0.158212,-3 10135\C,0,-6.340809,1.856595,-1.177086\H,0,-6.627319,2.138851,-0.1479 $01 \mathrm{H}, 0,-6.400306,2.761612,-1.809437 \mathrm{H}, 0,-7.088471,1.13323,-1.551686 \mathrm{IC}$, $0,-3.774533,-0.653682,4.247015 \backslash \mathrm{H}, 0,-4.548259,-1.419154,4.445163 \backslash \mathrm{H}, 0,-3$ $.111078,-0.60333,5.130273 \backslash \mathrm{H}, 0,-4.283267,0.320398,4.147841 \backslash \mathrm{C}, 0,-2.21290$ $7,-2.337861,3.177604 \backslash \mathrm{H}, 0,-1.484191,-2.236932,4.002867 \backslash \mathrm{H}, 0,-2.8961,-3.1$ $65669,3.4431 \backslash \mathrm{H}, 0,-1.65583,-2.604126,2.265903 \backslash \mathrm{C}, 0,2.444881,-0.444531,-4$ $.04607 \mathrm{VH}, 0,1.834644,-0.314285,-4.95896 \mathrm{H}, 0,3.337705,-1.032221,-4.32887$ $1 \mathrm{H}, 0,1.84874,-1.016054,-3.31655 \backslash \mathrm{H}, 0,1.875113,1.457129,-3.245014 \backslash \mathrm{C}, 0,3$ $.57705,-3.109218,2.226137 \backslash \mathrm{C}, 0,2.905449,-4.30674,2.522041 \backslash \mathrm{H}, 0,3.210082$, $-4.913086,3.379317 \backslash \mathrm{C}, 0,1.848517,-4.726475,1.71041 \mathrm{lH}, 0,1.329748,-5.6555$ $89,1.958629 \backslash \mathrm{C}, 0,1.442489,-3.948641,0.600768 \mid \mathrm{C}, 0,2.131864,-2.74359,0.31$ $8168 \mathrm{H}, 0,1.870065,-2.137691,-0.55503 \backslash \mathrm{C}, 0,3.188656,-2.327023,1.125832 \mathrm{H}$ $, 0,3.717206,-1.400798,0.88807 \backslash \mathrm{C}, 0,0.337532,-4.38652,-0.271363 \backslash \mathrm{C}, 0,-0.0$ $32042,-5.828658,-0.384952 \mathrm{H}, 0,-0.019952,-6.29377,0.613492 \mathrm{H}, 0,0.720615$ ,-6.360364,-0.995411\H,0,-1.02524,-5.965483,-0.835778\N,0,-0.298744,-3 $455419,-0.950094 \mathrm{|H}, 0,-0.089745,-2.46108,-0.712138 \backslash \mathrm{C}, 0,-1.277017,-3.58$ $0132,-1.980723 \backslash \mathrm{C}, 0,-1.118987,-4.497792,-3.03607 \backslash \mathrm{H}, 0,-0.24214,-5.149321$ $,-3.077019 \backslash \mathrm{C}, 0,-2.076289,-4.533869,-4.0580581 \mathrm{H}, 0,-1.953843,-5.242598,-$ $4.883579 \backslash \mathrm{C}, 0,-3.171584,-3.656342,-4.033541 \backslash \mathrm{C}, 0,-3.309396,-2.734209,-2$ $983386 \backslash \mathrm{H}, 0,-4.159085,-2.04745,-2.958843 \backslash \mathrm{C}, 0,-2.365077,-2.687187,-1.952$ $582 \mathrm{H}, 0,-2.477094,-1.980858,-1.125075 \mathrm{|H}, 0,-3.915123,-3.685407,-4.83691$ 9IC,0,4.714477,-2.641136,3.102026IF,0,5.297125,-3.658948,3.779686IF,0, $5.690759,-2.028637,2.380944 \mathrm{IF}, 0,4.298728,-1.73857,4.032671 \mid \mathrm{IVersion}=\mathrm{ES}$ $64 \mathrm{~L}-$ G09RevD.01 IState=1-AlHF=-3513.6052377 $\backslash \mathrm{RMSD}=4.196 \mathrm{e}-09 \mathrm{|Dipole}=-0.677$ $3552,-5.204177,-1.10719781 Q u a d r u p o l e=-20.2746366,41.2347341,-20.960097$ $5,0.1185029,1.4294712,8.9734658 \mathrm{PG}=\mathrm{C0} 01$ [X(C65H69F3N1O4P1)]1@ 


\section{References}

(1) Sorgenfrei, N.; Hioe, J.; Greindl, J.; Rothermel, K.; Morana, F.; Lokesh, N.; Gschwind, R. M. NMR Spectroscopic Characterization of Charge Assisted Strong Hydrogen Bonds in Brønsted Acid Catalysis. J. Am. Chem. Soc. 2016, 138 (50), 16345-16354.

(2) Greindl, J.; Hioe, J.; Sorgenfrei, N.; Morana, F.; Gschwind, R. M. Brønsted Acid Catalysis-Structural Preferences and Mobility in Imine/Phosphoric Acid Complexes. J. Am. Chem. Soc. 2016, 138 (49), 15965-15971.

(3) Aznar, F.; Valde, C. Modular Synthesis of Indoles from Imines and o-Dihaloarenes or o -Chlorosulfonates by a Pd-Catalyzed Cascade Process. J. Am. Chem. Soc. 2009, 131 (8), 4031-4041.

(4) Schramm, Y.; Barrios-Landeros, F.; Pfaltz, A. Discovery of an Iridacycle Catalyst with Improved Reactivity and Enantioselectivity in the Hydrogenation of Dialkyl Ketimines. Chem. Sci. 2013, 4, 2760-2766.

(5) Harris, R. K.; Becker, E. D.; Cabral de Menezes, S. M.; Goodfellow, R.; Granger, P. NMR Nomenclature: Nuclear Spin Properties and Conventions for Chemical Shifts. IUPAC Recommendations 2001. International Union of Pure and Applied Chemistry. Physical Chemistry Division. Commission on Molecular Structure and Spectroscopy. Magn. Reson. Chem. 2002, 40 (7), 489-505.

(6) Trigo-Mouriño, P.; Griesinger, C.; Lee, D. Label-Free NMR-Based Dissociation Kinetics Determination. J. Biomol. NMR 2017, 69 (4), 229-235.

(7) Akke, M.; Palmer, A. G. Monitoring Macromolecular Motions on Microsecond to Millisecond Time Scales by R $1 \rho-R 1$ Constant Relaxation Time NMR Spectroscopy. J. Am. Chem. Soc. 1996, 118 (4), 911-912.

(8) Palmer, A. G.; Kroenke, C. D.; Loria, J. P. Nuclear Magnetic Resonance Methods for Quantifying Microsecond-to-Millisecond Motions in Biological Macromolecules; Elsevier Masson SAS, 2001; Vol. 339.

(9) Melikian, M.; Gramüller, J.; Hioe, J.; Greindl, J.; Gschwind, R. M. Brønsted Acid Catalysis - the Effect of 3,3'-Substituents on the Structural Space and the Stabilization of Imine/Phosphoric Acid Complexes. Chem. Sci. 2019, 10, 5226-5234.

(10) Jerschow, A.; Müller, N. Suppression of Convection Artifacts in Stimulated-Echo Diffusion Experiments. Double-Stimulated-Echo Experiments. J. Magn. Reson. 1997, 125 (2), 372-375.

(11) Stejskal, E. O.; Tanner, J. E. Spin Diffusion Measurements: Spin Echoes in the Presence of a TimeDependent Field Gradient. J. Chem. Phys. 1965, 42 (1), 288-292.

(12) MacChioni, A.; Ciancaleoni, G.; Zuccaccia, C.; Zuccaccia, D. Determining Accurate Molecular Sizes in Solution through NMR Diffusion Spectroscopy. Chem. Soc. Rev. 2008, 37 (3), 479-489.

(13) Chen, H. C.; Chen, S. H. Diffusion of Crown Ethers in Alcohols. J. Phys. Chem. 1984, 88 (21), 51185121.

(14) Ben-Amotz, D.; Willis, K. G. Molecular Hard-Sphere Volume Increments. J. Phys. Chem. 1993, 97 (29), 7736-7742.

(15) Zuccaccia, D.; Macchioni, A. An Accurate Methodology to Identify the Level of Aggregation in Solution by PGSE NMR Measurements: The Case of Half-Sandwich Diamino Ruthenium(II) Salts. Organometallics 2005, 24 (14), 3476-3486.

(16) Reid, J. P.; Goodman, J. M. Goldilocks Catalysts: Computational Insights into the Role of the 3,3' Substituents on the Selectivity of BINOL-Derived Phosphoric Acid Catalysts. J. Am. Chem. Soc. 2016, 138 (25), 7910-7917.

(17) J. M. Tao; J. P. Perdew; V. N. Staroverov; G. E. Scuseria. Climbing the density functional ladder: Nonempirical meta-generalized gradient approximation designed for molecules and solids. Phys. Rev. Lett. 2003, 91, 146401.

(18) S. Grimme; J. Antony, S. Ehrlich; H. Krieg. A consistent and accurate ab initio parameterization of density functional dispersion correction (DFT-D) for the 94 elements H-Pu. J. Chem. Phys. 2010, 132, 154104.

(19) A. V. Marenich; C. J. Cramer; D. G. Truhlar. Universal solvation model based on solute electron density and a continuum model of the solvent defined by the bulk dielectric constant and atomic surface tensions. J. Phys. Chem. B, 2009, 113, 6378-6396. 
(20) S. Grimme. Improved second-order Møller-Plesset perturbation theory by separate scaling of parallel- and antiparallel-spin pair correlation energies. J. Chem. Phys., 2003, 118, 9095.

(21) Gaussian 09, Revision D.01, M. J. Frisch; G. W. Trucks; H. B. Schlegel; G. E. Scuseria; M. A. Robb; J. R. Cheeseman; G. Scalmani; V. Barone; B. Mennucci; G. A. Petersson; H. Nakatsuji; M. Caricato; X. Li; H. P. Hratchian; A. F. Izmaylov; J. Bloino; G. Zheng; J. L. Sonnenberg; M. Hada; M. Ehara; K. Toyota; R. Fukuda; J. Hasegawa; M. Ishida; T. Nakajima; Y. Honda; O. Kitao; H. Nakai; T. Vreven; J. A. Montgomery, Jr.; J. E. Peralta; F. Ogliaro; M. Bearpark; J. J. Heyd; E. Brothers; K. N. Kudin; V. N. Staroverov; R. Kobayashi; J. Normand; K. Raghavachari; A. Rendell; J. C. Burant; S. S. Iyengar; J. Tomasi; M. Cossi; N. Rega; J. M. Millam; M. Klene; J. E. Knox; J. B. Cross; V. Bakken; C. Adamo; J. Jaramillo; R. Gomperts; R. E. Stratmann; O. Yazyev; A. J. Austin; R. Cammi; C. Pomelli; J. W. Ochterski; R. L. Martin; K. Morokuma; V. G. Zakrzewski; G. A. Voth; P. Salvador; J. J. Dannenberg; S. Dapprich; A. D. Daniels; Ö. Farkas; J. B. Foresman; J. V. Ortiz; J. Cioslowski; D. J. Fox, Gaussian, Inc., Wallingford CT, 2009.

(22) F. Neese. Software update: the ORCA program system, version 4.0. WIREs Comput Mol Sci, 2018, 8:e1327. doi: $10.1002 /$ wcms.1327

(23) S. Grimme; C. Bannwarth; P. Shushkov. A Robust and Accurate Tight-Binding Quantum Chemical Method forStructures, Vibrational Frequencies, and Noncovalent Interactions ofLarge Molecular Systems Parametrized for All spd-Block Elements(Z=1-86). J. Chem. Theory Comput. 2017, 13, 1989. 